\title{
Field Ionization and Field Emission with \\ Intense Single-Cycle THz Pulses
}

\author{
Sha Li \\ Leshan, Sichuan, China \\ B.S. in Physics, Nanjing University, May 2009 \\ M.A. in Physics, University of Virginia, December 2012
}

\begin{abstract}
A Dissertation presented to the Graduate Faculty
of the University of Virginia in Candidacy for the Degree of Doctor of Philosophy
\end{abstract}

Department of Physics

University of Virginia

July 2016 
(C) Copyright by

Sha Li

All Rights Reserved

August 2016 


\begin{abstract}
This dissertation focuses on light-matter interactions in the strong-field, lowfrequency, single-cycle regime in both gas-phase and solid-state systems through the investigation of field ionization of Rydberg atoms and field emission from metallic nano-tips exposed to intense, single-cycle $\mathrm{THz}$ pulses. Field ionization/emission mechanism, as well as post-ionization/emission energy transfer dynamics, have been studied.

In the experiments, single-cycle THz pulses with a central frequency $f \sim 0.2 \mathrm{THz}$ and peak field amplitude $F_{m} \sim 0.5 \mathrm{MV} / \mathrm{cm}$ are used to explore:

1) Field ionization of excited atoms (Na Rydberg states of $n d, n=6-15$ ) in the long-pulse/low-frequency regime. A $F \propto n^{-3}$ over-the-barrier ionization threshold scaling behavior is observed, reflecting the suppression of core-scattering during the ionization pulse and, more importantly, the extended times required for Rydberg electrons to escape over the field-dressed Coulomb potential barrier. We have also examined energy transfer in the single-cycle limit and showed that in contrast to ionization by multi-cycle pulses, electrons that are ionized near the field extrema of a single-cycle pulse acquire substantial energies $\left(\sim 2 U_{p}\right)$, and those ionized near the zero-crossing can obtain much larger energies $\left(>6 U_{p}\right)$, even in the absence of rescattering.
\end{abstract}

2) Field ionization of oriented atoms (Na Rydberg-Stark states in an $n$-manifold, $n=10-12)$. The ionization threshold fields are found to be orientation-dependent, due to both diabatic population transfer between "uphill" and "downhill" oriented states and an asymmetry in the single-cycle $\mathrm{THz}$ waveform.

3) High-energy field-electron-emission from W nano-tips having different cone angles and tip radii, $10 \mathrm{~nm}<R<1 \mu \mathrm{m}$. Electrons with energies easily exceeding 
$5 \mathrm{keV}$ are observed. The maximum electron energies are proportional to the peak $\mathrm{THz}$ field and are roughly independent of the tip radius. These observations can be attributed to the large field enhancement and spatial localization of the enhanced field in the vicinity of the nano-tips. At low-frequencies/long-wavelengths, such near-field characteristics can fundamentally change the energy transfer process as compared to that which occurs following the ionization of gas-phase atoms or molecules, and allow for the creation of very high energy electrons. 


\section{Acknowledgments}

First and foremost, my thanks go to my parents, Mingxue Li and Lidong $\mathrm{Xu}$, for their unconditional love and support. My dad used to be my junior-high mathematics teacher, who guided me into the world of science. One of his big regrets is not being able to obtain higher-education when he was young, and he puts his hope on me. I am glad that I do not let him down. My mom sacrifices a lot to the family, from the moment I was born, until right now I am already a grown-up, she always takes the greatest care of me and is always my strongest backup, to her I own so much.

I would not be able to fulfill this work without the guidance of my advisor Prof. Bob Jones, to whom I want to express my sincere thanks. Thanks, Bob, not only for sharing your broad knowledge with me and putting up with all my questions, but also for teaching me the lab skills and patiently guiding me through the troubleshooting when I felt frustrated. I have learned from you not only how to be a scientist, but also a lot of things that are no doubt lifetime benefits. Besides, the thing that amazes me most is your ability of quickly explaining complicate science in a simply, yet very beautiful way. Because of this, I always tell others proudly that my advisor is a very smart and sharp scientist!

I am grateful to Prof. Tom Gallagher and Prof. Craig Group, who reviewed my work and kept my research on track annually. Thanks also go to Prof. Tom Gallagher, Prof. Lou Bloomfield and Prof. Patrick Hopkins for their serving in my Ph.D. defense committee.

My lab-mates helped me a lot during my Ph.D. years. Kelsie showed me how a femtosecond laser system worked when I joined the lab. And I could not remember how many times Tao, Brian and Kisra helped me lift heavy chamber pieces. I always enjoy working, discussing and chatting with them. 
I must thank all the staff members in the physics department. Their assistance make work in the department a lot easier.

I want to thank all my friends. Not only the ones here in Charlottesville, whose accompanies warm my life and make me feel less homesick, but also the ones that are far far away, sometime a phone call with them simply cheers me up.

My childhood idol was Marie Curie, and I dreamed of becoming a physicist when I was little. Now I look back at my Ph.D. life, there were times I felt great sense of achievements, and times I was quite frustrated. I realize that being a scientist is not that easy at all and there are always ups and downs. But I am glad that I overcome all the difficulties and take a step further towards my original dream. I thank myself for not giving up.

This work is supported by the US department of energy. 


\section{Contents}

Abstract $\quad$ ii

Acknowledgements $\quad$ iv

List of Tables viii

List of Figures $\quad$ ix

1 Introduction 1

1.1 Motivation ........................... 2

1.2 Rydberg atoms . . . . . . . . . . . . . . . . . . 2

1.2.1 Schröedinger equation of atomic hydrogen . . . . . . . . . . 3

1.2.2 Quantum defect theory . . . . . . . . . . . . . . 7

1.2 .3 (DC) Stark effect . . . . . . . . . . . . . . 8

1.3 Strong field physics:

Ionization and post-ionization dynamics . . . . . . . . . . . . . 13

1.3.1 Light induced atomic ionization . . . . . . . . . . . . . 13

1.3.2 Post-ionization dynamics . . . . . . . . . . . . . . . . . . . . 19

1.3.3 Strong field physics in nanostructured solids . . . . . . . . . . 22

$1.4 \mathrm{THz}$ radiation . . . . . . . . . . . . . . . . 26 
2 Experimental setups and concepts 31

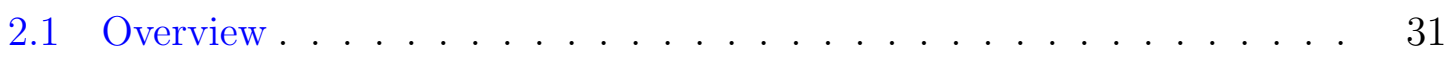

2.2 Femtosecond laser system _. . . . . . . . . . . . . . . . . . . 32

$2.3 \mathrm{THz}$ generation and characterization . . . . . . . . . . . 33

2.3.1 Optical rectification . . . . . . . . . . . . . . 33

2.3.2 Electro-optic sampling . . . . . . . . . . . . . . . . . 38

2.3.3 Streaking spectroscopy . . . . . . . . . . . . . . 43

2.4 Dye Lasers . . . . . . . . . . . . . . . . . . . . . 46

2.5 Photoelectron spectroscopy _. . . . . . . . . . . . . . 47

2.5.1 Time-of-flight electron spectrometer . . . . . . . . . . . . 47

2.5.2 Retarding potential energy analyzer . . . . . . . . . . . . . 49

2.6 Sample preparation . . . . . . . . . . . . . . . 50

2.6.1 Sodium Oven .................... 50

2.6.2 Tungsten nano-tips .................. 50

2.7 Vacuum System . . . . . . . . . . . . . . . . 54

3 Ionization of Excited Atoms by Intense Single-Cycle THz Pulses 57

4 Ionization of Oriented Atoms by Intense, Single-Cycle THz Pulses 78

5 High-Energy Electron Emission from Metallic Nano-tips Driven by $\begin{array}{ll}\text { Intense Single-Cycle THz Pulses } & 92\end{array}$

6 Conclusion and Outlook 111

$\begin{array}{ll}\text { Bibliography } & 114\end{array}$

$\begin{array}{lr}\text { Appendices } & 129\end{array}$ 
$\begin{array}{ll}\text { A Atomic units } & 130\end{array}$

B Coordinates 132

$\begin{array}{ll}\text { C Kinematics of rescattering } & 133\end{array}$

$\begin{array}{ll}\text { D Femtosecond laser system } & 136\end{array}$

$\begin{array}{lrl}\text { E Electro-optic sampling } & 142\end{array}$

F Time-of-flight spectrometer and retarding potential energy analyzer 148

G Circuit diagram of the fast switch for electrochemical etching 153

$\begin{array}{ll}\text { H Landau-Zener transition } & 154\end{array}$

I Calibration of the unsaturated ionization probability 156

$\begin{array}{lll}\text { J Simulation of the field-emission electron energy spectra } & 158\end{array}$ 


\section{List of Tables}

1.1 Quantum defect parameters for Na. . . . . . . . . . . 8

1.2 Values of relevant parameters for the ionization of ground state hydrogen with XUV, NIR or $\mathrm{THz}$ radiation. . . . . . . . . . . . . . 18

A.1 Values of fundamental constants in atomic and SI units. . . . . . . . 130

A.2 Derived atomic units. . . . . . . . . . . . . . . . . . 131 


\section{List of Figures}

1.1 Classical Kepler motion of a hydrogenic electron. . . . . . . . . . . 5

1.2 Projection of the zero-field parabolic states on the zero-field d state. . 6

1.3 Visualization of the potentials $V(\xi)$ and $V(\eta)$ for $F=10^{-6}$ (a.u.). . . 10

1.4 A demostration of the Stark energy levels for $\mathrm{H}$ and Na. . . . . . . . 10

1.5 Charge distribution for the hydrogenic parabolic eigenstates in a plane containing the $z$ axis. . . . . . . . . . . . . . . 13

1.6 Photoionization and field ionization. . . . . . . . . . . . . . 14

1.7 Schematic of different ionization regimes based on the Keldysh theory. 18

1.8 The three-step model. . . . . . . . . . . . . . . . . . . . . . . . . 20

1.9 Illustration of the phase dependence in the rescattering process. . . . 21

1.10 Optical liberation of electrons from a metal. . . . . . . . . . . . 22

1.11 Geometry of a hemisphere capped cylindrical field emitter. . . . . . . 25

1.12 Electromagnetic spectrum and characteristics of $\mathrm{THz}$ radiation. . . . 26

1.13 Methods of generating $\mathrm{THz}$ radiation. . . . . . . . . . . . . . . 29

1.14 Coherent detection of $\mathrm{THz}$ radiation. . . . . . . . . . . . . . 30

2.1 Principle of $\mathrm{THz}$ generation via optical rectification . . . . . . . . . 34

2.2 Phase matching via tilted-pulse-front-pumping. . . . . . . . . . . 36 
2.3 Schematic of the experimental setup for $\mathrm{THz}$ generation via TPFP opcical rectification. . . . . . . . . . . . . . . . . . . . . . . . 38

2.4 Principle of electro-optic sampling. . . . . . . . . . . . . . . . . . . 39

2.5 Electro-optic sampling experimental setup. . . . . . . . . . . . . 41

2.6 Electro-optic sampling experimental results. . . . . . . . . . . . . . . 42

2.7 NIR-XUV optical streaking light field measurement: Principle. . . . . 45

2.8 NIR-XUV optical streaking light field measurement: Streaked electron energies and derived NIR light field. . . . . . . . . . . . . . . . . 45

2.9 Schematics of Hänsch and Littman dye lasers. . . . . . . . . . . . . . 46

2.10 Electrochemical etching setup and operation principle. . . . . . . . 52

2.11 Electrochemical etching photo view and demonstration of mounting the wires on the SEM sample holder. . . . . . . . . . . . . . 53

2.12 SEM images of electrochemically etched tungsten nano-tips. . . . . . 53

2.13 Schematic of the vacuum system exterior. . . . . . . . . . . . 55

2.14 Schematic of setups inside the vacuum chamber, top view. . . . . . . 56

2.15 Schematic of the interaction region, side view. . . . . . . . . . 56

3.1 Classical over-the-barrier ionization for hydrogen and non-hydrogenic

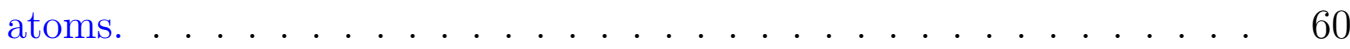

3.2 An example of microwave field ionization of non-hydrogenic Rydberg

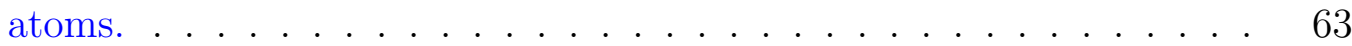

3.3 Ionization by a half-cycle pulse. . . . . . . . . . . . . . . . . . 64

3.4 Photoelectron streaking results and the experimental setup. . . . . . 67

3.5 Measured ionization probability as a function of $F_{\max }$ for several $n d$ states. . . . . . . . . . . . . . . . 70

3.6 Ionization threshold field scaling behavior. . . . . . . . . . . . . . . 70 
3.7 Electron energy distributions for different $n$-states ionized by a $F_{m}=$ $430 \mathrm{kV} / \mathrm{cm}$ single-cycle THz field. . . . . . . . . . . . . . . . 73

3.8 Theoretical study of single-cycle field ionization of Rydberg atoms in both long-pulse and short-pulse regimes. . . . . . . . . . . . 77

4.1 THz waveform, schematic of the interaction region and schematic of a Stark manifold. . . . . . . . . . . . . . . . . . . 80

4.2 Measured ionization probability as a function of peak THz field strength for "uphill" and "downhill" Stark states. . . . . . . . . . . . . . 82

4.3 Peak THz field required for $10 \%$ ionization as a function of $k$ within different $n$-manifolds. . . . . . . . . . . . . . . . . . . 82

4.4 Dynamics of single-cycle ionization of oriented Rydberg-Stark states. $\quad 86$

4.5 Illustration of single-cycle $\mathrm{THz}$ driven electron scattering from RydbergStark eigenstates. . . . . . . . . . . . . . . . . . . . . 89

5.1 THz induced field emission from $\mathrm{W}$ nano-tips: Experimental setup and

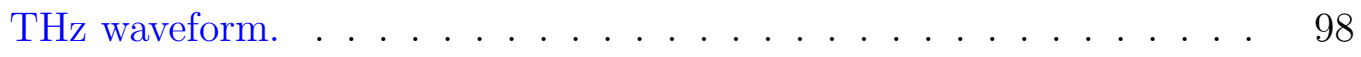

5.2 Results of THz induced field emission from a tip of radius $R \simeq 130 \mathrm{~nm} .99$

5.3 Comparison of $\mathrm{THz}$ induced field emission from different tips. . . . . 101

5.4 SEM images of a W nanotip before and after tens of hours of intense THz exposure in vacuum. . . . . . . . . . . . . . 106

5.5 THz-induced field emission from Au multi-nanotipped wire: Experimental data. . . . . . . . . . . . . . . . . . . 108

5.6 THz-induced field emission from Pt STM-cut wire: Experimental data. 108

5.7 Dependence of the electron current $J$ on incident THz field strength $F$ plotted using standard Fowler-Nordheim scaled parameters. . . . . . . 110

5.8 SEM images of a Au multi-nanotipped wire and a Pt STM-cut wire. . 110 
D.1 Schematic of the single-shot autocorrelation and its operation. . . . . 139

D.2 Ti:sapphire oscillator and sample mode-locked output spectrum. . . . 139

D.3 Ti:sapphire chirped pulse amplifier. . . . . . . . . . . . . . . . . 140

D.4 Grating based pulse stretcher, compressor and spectrometer. . . . . . 141

E.1 The THz field modified the index ellipse in the (110) plane. . . . . . . 144

F.1 Time of flight electron spectrometer and retarding potential analyzer. 149

F.2 TOF electron spectrometer calibration results. . . . . . . . . . . . . 150

F.3 Resolution of the LeCroy 9350A oscilloscope. . . . . . . . . . . . . . . 151

G.1 Circuit diagram of the fast switch for electrochemical etching. . . . . 153

H.1 Two-level system with an avoided energy crossing $\Delta E . \ldots . . . . .154$

I.1 Calibration of the ionization probability for a state whose ionization is below saturation. . . . . . . . . . . . . . . . . 157

J.1 Hyperboloidal model for field spatial decay and hemisphere-capped cylinder model for field enhancement factor. . . . . . . . . . . . . . . 160

J.2 Simulation results of field-emission electron energy spectra. . . . . . 160 


\section{Chapter 1}

\section{Introduction}

This chapter provides the motivation for the work described in this dissertation, as well as some background information, including: introductions to Rydberg atoms and $\mathrm{THz}$ radiation, and a brief review of strong field light-matter interaction, particularly, ionization and postionization dynamics, for both gas phase atoms and nano-structured solids.

Atomic units (a. u.), in which the fundamental constants of elementary charge $e$, electron rest mass $m_{e}$, reduced Planck constant $\hbar$, and Coulomb's constant $k_{e}=$ $1 /\left(4 \pi \varepsilon_{0}\right)$ are defined to be unity: $e=m_{e}=\hbar=k_{e} \equiv 1$, are used in this dissertation unless specified otherwise. A brief description of the atomic units system can be found in Appendix A. 


\subsection{Motivation}

The goal of this dissertation is to explore light-matter interactions in the strong-field, low-frequency, single-cycle regime in both gas-phase and solid-state systems. Here "low-frequency" refers to the $\mathrm{THz}$ frequency regime. $\mathrm{THz}$ radiation has a frequency that falls in between that of infrared and microwave radiations. In contrast to optical waves, $\mathrm{THz}$ radiation allows direct access to numerous fundamental processes that resonate at low frequencies. Strong THz fields also enable the study of non-resonant light-matter interactions, for example, field ionization of atomic and molecular gases, field emission from solids, manipulation of high-energy electron trajectories, nonlinear modulation of the optical properties of solids, etc. In our laboratory, we are able to generate intense, single-cycle $\mathrm{THz}$ pulses with peak field strengths as high as $F_{m} \sim 0.5$

$\mathrm{MV} / \mathrm{cm}$. With such high $\mathrm{THz}$ fields, we can investigate field ionization of Rydberg atoms and field emission from metallic nano-tips. A better understanding of the field ionization/emission dynamics, as well as the post-ionization/emission energy transfer process, has been obtained.

\section{$1.2 \quad$ Rydberg atoms}

Rydberg atoms and molecules are named for the physicist Johannes Rydberg, who, followed the studies of Liveing and Dewar [1], Hartley [2], and Balmer [3], classified series of atomic spectral lines in 1889 [4]. Rydberg atoms and molecules have one or more valence electron(s) in highly excited state(s) with large principle quantum number $n$. Part of the research described in this dissertation involves Na Rydberg atoms which have a single valence electron. So, unless specified otherwise, the discussion here is limited to Rydberg atoms with one valence electron. 
Rydberg atoms are very beautiful quasi-one electron systems. They have many exaggerated properties and are excellent choices for exploring dynamics at the atomic level. Highly excited hydrogen is the simplest example of a Rydberg atom. Nonhydrogenic Rydberg atoms (for example, alkali metal atoms) are similar to the hydrogen atom in the respect that the valence electron spends most of its time far away from the ionic core and experiences effectively a net +1 ionic core charge. Differences arise, however, due to the finite core size of non-H Rydberg atoms, and the imperfect shielding of the nucleus by the core electrons. The non-hydrogenic effects can be treated using quantum defect theory, as will be discussed later.

\subsubsection{Schröedinger equation of atomic hydrogen}

In 1913 Bohr proposed his model for the hydrogen atom [5]. Today we know that the Schröedinger equation of atomic hydrogen, or more generally, hydrogen-like ions with Coulomb potential $V(r)=-Z / r$ where $Z$ is the net charge of the ion to which the Rydberg electron is bound, is analytically solvable. The bound state eigenenergies and normalized eigenfunctions (of the time-independent Schröedinger equation) in spherical coordinates $(r, \theta, \phi)$ are [6]:

$$
\begin{aligned}
E_{n} & =-\frac{Z^{2}}{2 n^{2}}=-Z^{2} \frac{R y_{\infty}}{n^{2}} \quad n=1,2,3, \cdots \\
\psi_{n l m}(r, \theta, \phi) \equiv|n l m\rangle & \underbrace{\sqrt{\left(\frac{2 Z}{n}\right)^{3} \frac{(n-l-1) !}{2 n[(n+l) !]^{3}}\left(\frac{2 Z}{n} r\right) e^{l} e^{-\frac{Z}{n} r} L_{n-l-1}^{2 l+1}\left(\frac{2 Z}{n} r\right)}}_{R_{n l}(r) ; l=0,1, \cdots, n-1} \\
& \times \underbrace{(-1)^{m} \sqrt{\frac{(2 l+1)}{4 \pi} \cdot \frac{(l-m) !}{(l+m) !}} P_{l}^{m}(\cos \theta) e^{i m \phi}}_{Y_{l}^{m}(\theta, \phi) ; m=0, \pm 1, \cdots, \pm l}
\end{aligned}
$$


Here $R y_{\infty}=1 / 2$ is the Rydberg constant, and $L_{\alpha}^{\beta}(x)$ and $P_{\alpha}^{\beta}(x)$ are the associated Laguerre and Legendre polynomials, respectively. Taking into account the finite mass of the nucleus, the energy is corrected to,

$$
E_{n}=-Z^{2} \frac{R y}{n^{2}}
$$

where $R y=R y_{\infty} /\left(1+m_{e} / m_{\text {core }}\right)$ is the Rydberg constant for the atom under study.

The $|n l m\rangle$ are simultaneous eigenstates of $\left[\hat{H}, L^{2}, L_{z}\right]$, where $n, l, m$ are the principal, azimuthal, and magnetic quantum number, respectively. $R_{n l}(r)$ and $Y_{l}^{m}(\theta, \phi)$ are the radial and angular wavefunctions, respectively. The electron dynamics can be described by the time-dependent wavefunctions composed of superpositions of eigenstates with different energies.

Since the attractive Coulomb potential is inversely proportional to $r$, the radial motion at constant angular momentum is classically analogous to Kepler planetary motion, where $[7,8]$ :

$$
r=\frac{L^{2} / Z}{1+(|\mathbf{A}| / Z) \cos \theta}=\frac{l(l+1) / Z}{1+\sqrt{1-l(l+1) / n^{2}} \cos \theta}
$$

The angular momentum $\mathbf{L}=\mathbf{r} \times \mathbf{p}$ and the Runge-Lenz vector $\mathbf{A}=\mathbf{p} \times \mathbf{L}-Z \hat{\mathbf{r}}$ are conserved quantities of this Coulombic system (Fig. 1.1c): $L^{2}=l(l+1), A^{2}=$ $Z^{2}+2 E_{n} L^{2}$. During classical Kepler orbital motion, a Rydberg electron spends most of its time far away from the ionic core. This is reflected by the density of dots in Fig. 1.1a, depicting the classical electron's position as computed at equal time intervals [8]. For a given energy $E_{n}=-\frac{Z^{2}}{2 n^{2}}$, the orbital radius and period scale as $n^{2}$ and $n^{3}$ respectively, and the eccentricity $\varepsilon=\sqrt{1-l(l+1) / n^{2}}$ of the orbit is determined by $l$. Low $l$ orbits are more elliptical with higher probabilities of finding the electron near the core (see Fig. 1.1b, for $n \gg l$, the inner turning point of the orbit is $r_{\text {inner }} \simeq \frac{l(l+1)}{2 Z}$ ). The magnetic quantum number $m$ defines the orientation of the orbital plane relative 
to the $\mathrm{z}$ axis, i.e., the $m=l$ orbits lie in the $x y$ plane whereas the $m=0$ orbits define a plane containing the $z$-axis (Fig. 1.1c).

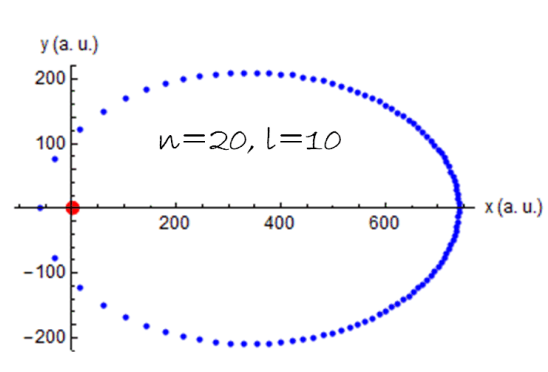

a

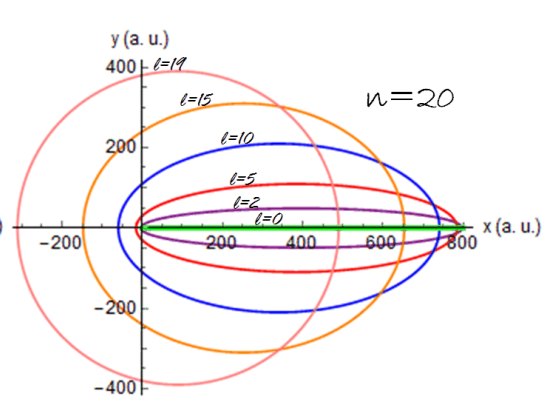

b

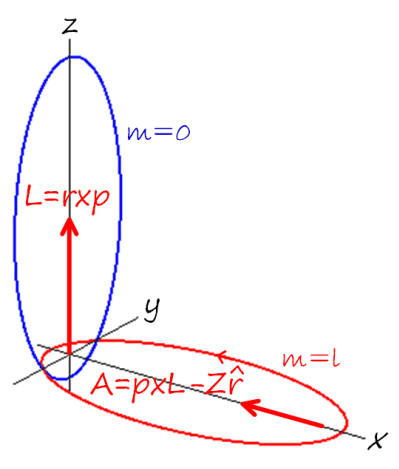

c

Figure 1.1: Classical Kepler motion of a hydrogenic electron.

The hydrogenic Schrödinger equation is also separable in parabolic coordinates $(\xi, \eta, \phi),\left(\xi=r+z, \eta=r-z, \phi=\arctan \frac{y}{x}\right)$, where the bound state solutions are $[9]:$

$$
\begin{aligned}
E_{n_{1} n_{2} m} & =-\frac{\epsilon^{2}}{2} \quad \epsilon=Z\left(n_{1}+n_{2}+|m|+1\right)^{-1}=Z / n \\
\psi_{n_{1} n_{2} m}(\xi, \eta, \phi) \equiv & \left|n_{1} n_{2} m\right\rangle \\
= & \sqrt{\frac{\epsilon^{(2|m|+4)} n_{1} ! n_{2} !}{2 \pi Z\left(n_{1}+|m|\right) !\left(n_{2}+|m|\right) !}} \\
& \times e^{-\frac{1}{2} \epsilon(\xi+\eta)}(\xi \eta)^{|m| / 2} L_{n_{1}}^{|m|}(\epsilon \xi) L_{n_{2}}^{|m|}(\epsilon \eta) e^{i m \phi}
\end{aligned}
$$

The $\left|n_{1} n_{2} m\right\rangle$ are simultaneous eigenstates of $\left[\hat{H}, L_{z}, A_{z}\right]$, where $A_{z}$ is the projection of the Runge-Lenz vector on the z-axis. $n_{1}$ and $n_{2}$ are called the parabolic quantum numbers, which are non-negative integers satisfying $n_{1}+n_{2}+|m|+1=n$ $\left(0 \leq n_{1}, n_{2} \leq n-|m|-1\right)$. Alternatively, $k=\left(n_{1}-n_{2}\right) \in\{-(n-|m|-1),-(n-|m|-$ 3), $\cdots, n-|m|-3, n-|m|-1\}$ can be defined as the parabolic quantum number. In this case, $n_{1}=\frac{n-|m|-1+k}{2}, n_{2}=\frac{n-|m|-1-k}{2}$, and $\left|n_{1} n_{2} m\right\rangle \equiv|n k m\rangle$. The coefficients 
for the transformation between the parabolic and spherical bases are Clebsch-Gordon coefficients which can be given in terms of the Wigner 3j-symbols [10],

$$
\begin{gathered}
|n k m\rangle=\sum_{l}|n l m\rangle\langle n l m \mid n k m\rangle \\
\langle n k m \mid n l m\rangle=(-1)^{[(1-n-m+k) / 2]+l} \times \sqrt{2 l+1}\left(\begin{array}{ccc}
\frac{n-1}{2} & \frac{n-1}{2} & l \\
\frac{m+k}{2} & \frac{m-k}{2} & -m
\end{array}\right)
\end{gathered}
$$

Fig. 1.2 shows the projection of the (zero-field) parabolic states $|n k m\rangle$ on the (zero-field) spherical $l=2$, d state. As shown, for $m=0$, the highest $|k|$ parabolic state has the most $\mathrm{d}$ character, whereas for $m=2$, the highest $|k|$ parabolic state has the least $\mathrm{d}$ character (or vanishing $\mathrm{d}$ character for large $\mathrm{n}$ ). It is also interesting to point out that as $\langle n k m \mid n 0 m\rangle=\sqrt{1 / n}=$ const, the $l=0$, s state is evenly spread over the $|n k m\rangle$ parabolic states of the $n$ manifold.
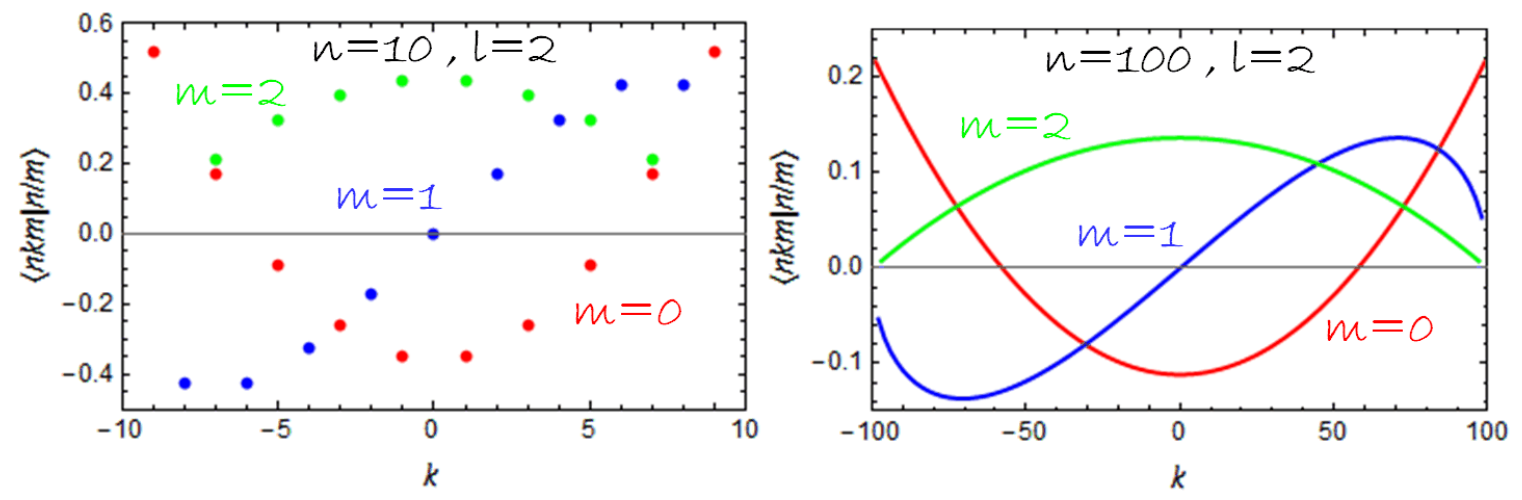

Figure 1.2: Projection of the zero-field parabolic states $|n k m\rangle$ on the zerofield d state, reproduce follows Ref. [10].

As will be discussed in more detail later, the parabolic basis is extremely useful when treating the "Stark" effect, which describes the influence of an external electric field on atoms. Notably, for hydrogen, the Schrödinger equation remains separable in parabolic coordinates even in the presence of an external electric field. 


\subsubsection{Quantum defect theory}

(Single channel) quantum defect theory (QDT) assumes that the effective core of a non-H Rydberg atom consists of a nucleus of charge $+Z$ symmetrically shielded by a spherical cloud of $Z-1$ electrons, and is "frozen" in space with a finite core size of radius $r=r_{c}$. The principal effects of the finite sized core, core polarization and penetration, are characterized by the quantum defect $[10,11]$. The rigorous quantum defect theory developed by Seaton [12] shows that the radial wavefunction of a non- $\mathrm{H}$ Rydberg electron experiences a phase shift upon scattering form the ionic core,

$$
\tau=\pi \delta_{l}
$$

In addition, the energy of each Rydberg state is modified,

$$
E_{n}=-\frac{R y}{\left(n-\delta_{l}\right)^{2}}
$$

where $\delta_{l}$ is the $l$-dependent quantum defect. In general, low $l$ states are influenced more strongly by the ionic core and, therefore, have larger quantum defects. Accurate measurements also reveal an n-dependence of the quantum defect, which is conveniently parameterized in a modified Rydberg-Ritz expression. For a specific $n l j$ state [10],

$$
\delta_{n l j}=\delta_{0}+\frac{\delta_{2}}{\left(n-\delta_{0}\right)^{2}}+\frac{\delta_{4}}{\left(n-\delta_{0}\right)^{4}}+\frac{\delta_{6}}{\left(n-\delta_{0}\right)^{6}}+\frac{\delta_{8}}{\left(n-\delta_{0}\right)^{8}}+\cdots
$$

Tab. 1.1 lists the parameters $\delta_{i=0,2}, \ldots$ for Na. As shown, s and $\mathrm{p}$ states have the largest quantum defects, whereas the $l=2$, d-states already have very small quantum defects $\delta_{l=2} \simeq 0.016$. 
Table 1.1: Quantum defect parameters for Na, from Ref. [10].

\begin{tabular}{|c|c|c|c|c|}
\hline & $\delta_{0}$ & $\delta_{2}$ & $\delta_{4}$ & $\delta_{6}$ \\
\hline$s_{1 / 2}$ & $1.3479692(4)$ & $0.06137(10)$ & & \\
\hline$p_{1 / 2}$ & $0.855424(6)$ & $0.1222(2)$ & & \\
\hline$p_{3 / 2}$ & 0.854608 & $0.1220(2)$ & & \\
\hline$d_{3 / 2,5 / 2}$ & 0.015543 & -0.08535 & 0.7958 & -4.0513 \\
\hline$f_{5 / 2,7 / 2}$ & $0.001663(60)$ & $-0.0098(3)$ & & \\
\hline
\end{tabular}

\subsection{3 (DC) Stark effect}

The influence of an external static electric field on Rydberg atoms is described by the DC Stark effect. The static field breaks the spherical symmetry of the atom and hence the $l m$ degeneracy of the energy eigenstates, and eigenstates with permanent electric dipole moments are formed.

An electric field $F$ applied in the $\boldsymbol{z}$ direction produces a dipole potential energy,

$$
V_{F}(z)=F z=F r \cos \theta
$$

To a first order approximation, we can ignore the coupling among states of different $\mathrm{n}$ and only consider the Hamiltonian for states of a particular $\mathrm{n}$. In this case, the matrix elements of the Hamiltonian in spherical basis $|n l m\rangle$ for hydrogen are:

$$
\begin{gathered}
\left\langle n l m\left|\left(\hat{H}_{0}+\hat{V}_{F}\right)\right| n l^{\prime} m^{\prime}\right\rangle=\left\langle n l m\left|\hat{H}_{0}\right| n l^{\prime} m^{\prime}\right\rangle+F\left\langle n l m|r \cos \theta| n l^{\prime} m^{\prime}\right\rangle \\
=-\frac{1}{2 n^{2}} \delta_{l l^{\prime}} \delta_{m m^{\prime}}-\frac{3 F n}{2} \delta_{m m^{\prime}}\left[\sqrt{\frac{\left(n^{2}-\left(l^{\prime}-1\right)^{2}\right)\left(l^{2}-m^{2}\right)}{\left(2 l^{\prime}+1\right)\left(2 l^{\prime}-1\right)}} \delta_{l, l^{\prime}-1}+\sqrt{\frac{\left(n^{2}-(l-1)^{2}\right)\left(l^{2}-m^{2}\right)}{(2 l+1)(2 l-1)}} \delta_{l, l^{\prime}+1}\right]
\end{gathered}
$$

Clearly $m$ is still a good quantum number and states of different $m$ are not coupled. The non-zero matrix elements include the diagonal elements, which are the 
common energies $-1 /\left(2 n^{2}\right)$, and the off diagonal elements $\left\langle n l m\left|\hat{V}_{F}\right| n(l \pm 1) m\right\rangle \propto F$. If we subtract off the common energy, all the entries of the Hamiltonian matrix are proportional to $F$ and, therefore, all the eigenvectors are independent of $F$ and the eigenvalues are proportional to $F$. This means that the wavefunctions of the eigenstates are field independent, and can be expanded as linear combinations of the zero field $|n l m\rangle$ spherical eigenstates. Moreover, the states exhibit linear energy shifts $\Delta E$, and exhibit permanent electric dipole moments, $\mu=-\langle z\rangle=-\Delta E / F[10]$.

Diagonalizing the Hamiltonian for the manifold of states associated with the same $n$ is essentially the same as solving the Schröedinger equation in parabolic coordinates as, to lowest order in perturbation theory, it remains separable in parabolic coordinates even with an external electric field. We have already shown the zero-field parabolic solutions. Generally, the eigenstates can be written as the product $[10,11]$,

$$
\psi(\xi, \eta, \phi)=\frac{1}{\sqrt{2 \pi \xi \eta}} \chi_{1}(\xi) \chi_{2}(\eta) e^{i m \phi}
$$

where $\chi_{1}(\xi)$ and $\chi_{2}(\eta)$ satisfy:

$$
\begin{array}{ll}
-\frac{1}{2} \frac{\mathrm{d}^{2} \chi_{1}}{\mathrm{~d} \xi^{2}}+\frac{V(\xi)-E}{4} \chi_{1}=0, & V(\xi)=2\left(-\frac{Z_{1}}{\xi}+\frac{m^{2}-1}{4 \xi^{2}}+\frac{F \xi}{4}\right) \\
-\frac{1}{2} \frac{\mathrm{d}^{2} \chi_{2}}{\mathrm{~d} \eta^{2}}+\frac{V(\eta)-E}{4} \chi_{2}=0, & V(\eta)=2\left(-\frac{Z_{2}}{\eta}+\frac{m^{2}-1}{4 \eta^{2}}-\frac{F \eta}{4}\right)
\end{array}
$$

Here $Z_{1}$ and $Z_{2}$ are separation constants and are related by $Z_{1}+Z_{2}=1$, assuming an ionic core with +1 net charge. At zero field, $Z_{1,2}=\frac{1}{n}\left(n_{1,2}+\frac{|m|+1}{2}\right) \cdot \chi_{1}$ and $\chi_{2}$ are the wavefuctions for an electron of energy $E / 4$ in the potentials $V(\xi) / 4$ and $V(\eta) / 4$, respectively. It is interesting to note that at zero field, Eq. 1.14 and Eq. 1.15 are similar to the radial equation for the Coulomb potential in the spherical coordinates. From this point of view, $Z_{1}$ and $Z_{2}$ may be thought as the positive charges binding the electron in the $\xi$ and $\eta$ coordinates. Fig. 1.3 shows the shape of the potentials 
$V(\xi)$ and $V(\eta)$ for $F=10^{-6}$ a.u. and for various choice of $Z_{1}$ and $Z_{2}$. Clearly $V(\xi) \rightarrow F \xi \rightarrow \infty$ and $V(\eta) \rightarrow-F \eta \rightarrow-\infty$ as $\xi, \eta \rightarrow \infty$. Thus, the eigenfuctions in the $\xi$ coordinate are bounded and can be described by a wavefunction with a well defined quantum number $n_{1}$. In the $\eta$ coordinate the wavefunctions are unbounded at any energy. The eigenstates have approximate good quantum numbers $n_{2}$ only at energies far below the saddle point of $V(\eta)$, where tunneling is negligible [10].

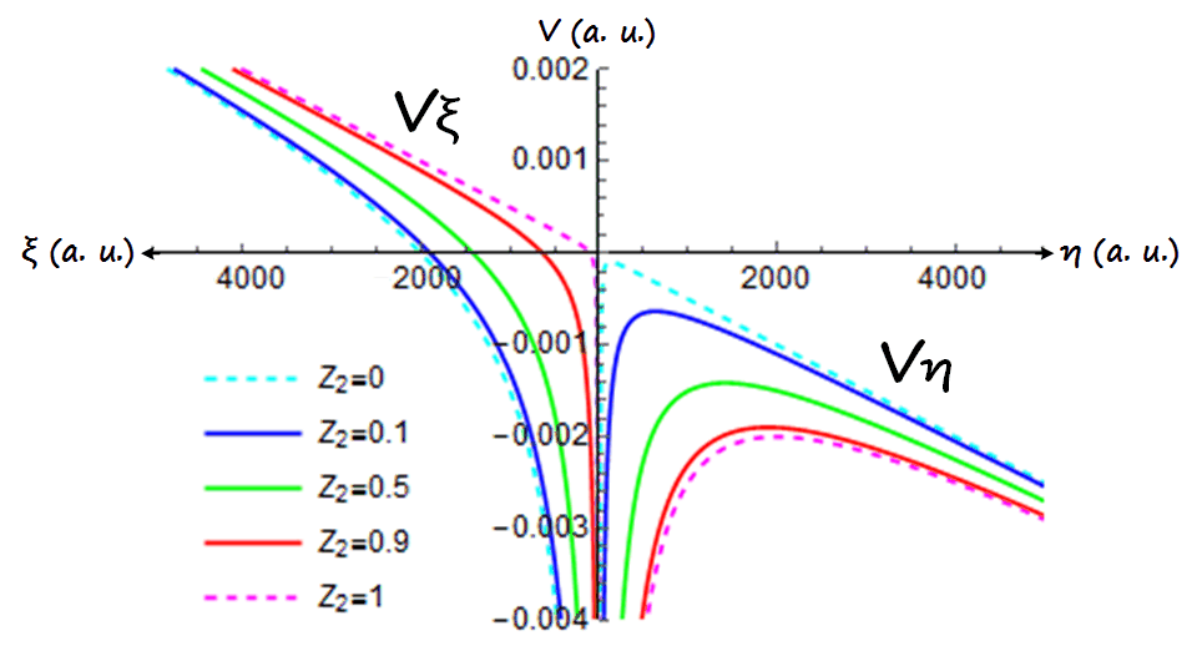

Figure 1.3: Visualization of the potentials $V(\xi)$ and $V(\eta)$ for $F=10^{-6}$ a.u.
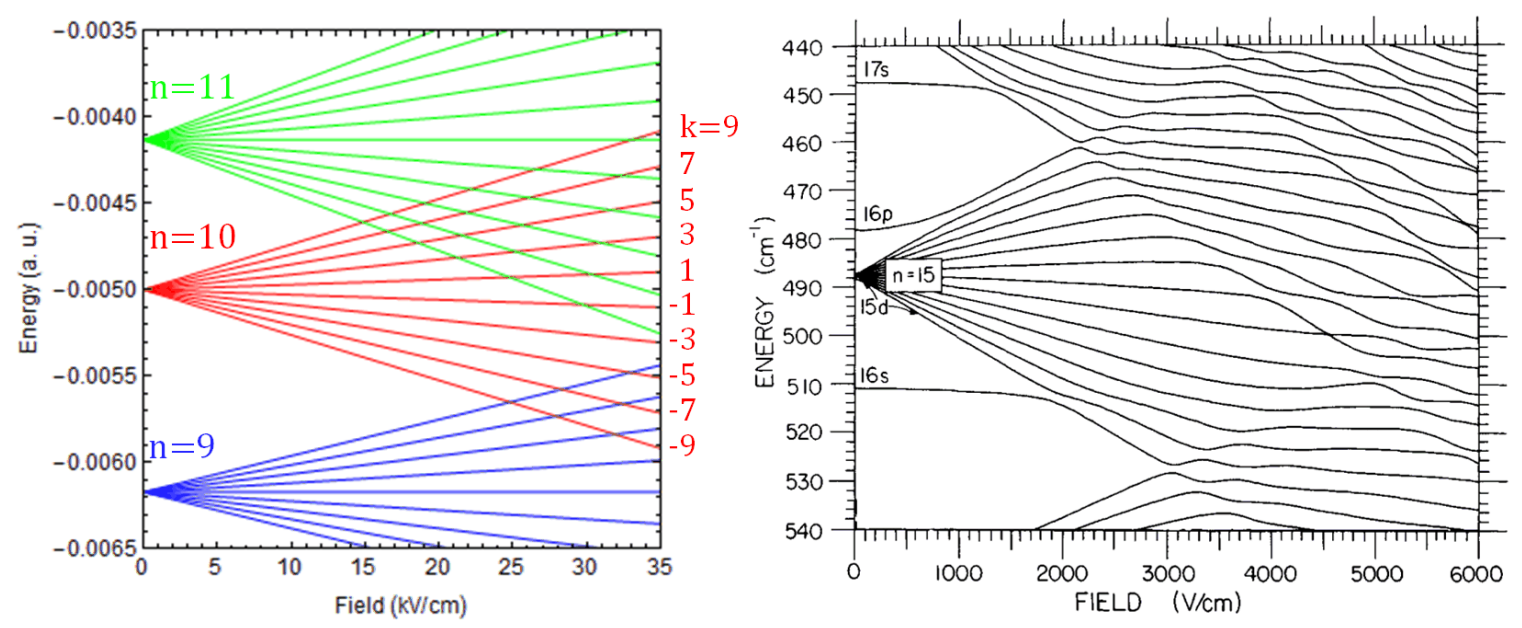

Figure 1.4: Left) Stark energy levels of $\mathrm{H} n=9,10,11, m=0$ manifolds of states. Right) Stark energy levels of Na $m=0$ states (from Ref. [10]). The $n=15$ manifold is shown. 
Eq. 1.14 and Eq. 1.15 can be solved perturbatively, numerically, or through the approximate WKB methods. The field-dependent energies for hydrogen, up to $5^{\text {th }}$ order in perturbation theory, are [11],

$$
\begin{aligned}
E_{n k m}= & -\frac{1}{2 n^{2}} \\
& +\frac{3}{2} n k F \\
& -\frac{n^{4}}{16}\left(17 n^{2}-3 k^{2}-9 m^{2}+19\right) F^{2} \\
& +\frac{3}{32} n^{7} k\left(23 n^{2}-k^{2}+11 m^{2}+39\right) F^{3} \\
& -\frac{n^{10}}{1024}\left(5487 n^{4}+35182 n^{2}-1134 m^{2} k^{2}+1806 n^{2} k^{2}-3402 n^{2} m^{2}+147 k^{4}\right. \\
& \left.\quad-549 m^{4}+5754 k^{2}-8622 m^{2}+16211\right) F^{4} \\
& +\frac{3}{1024} n^{13} k\left(10563 n^{4}+90708 n^{2}+220 m^{2} k^{2}+98 n^{2} k^{2}+772 n^{2} m^{2}-21 k^{4}\right. \\
& \left.\quad+725 m^{4}+780 k^{2}+830 m^{2}+59293\right) F^{5}+O\left(F^{6}\right)
\end{aligned}
$$

The energy shift is usually known as the Stark shift. As expected, the first order Stark shift is proportional to the electric field $F$. The second order Stark shift breaks the $m$ degeneracy, and is always to lower energy, as can be understood from oscillator strength sum rules [13]. For many applications, knowing the first and second order shifts is sufficient. Fig. 1.4Left is a plot of the Stark energies as a function of the electric field for hydrogen $n=9,10,11, m=0$ manifolds of states. It clearly shows the nearly linear dependence of the energy on the relatively weak field, and the energy crossings for states of different $n$ as they exhibit very weak couplings.

For non-H Rydberg atoms, the non-zero quantum defects results in zero-field energy splittings. For states of the same $n$, lower $l$ states haver larger splittings. In addition, with a finite sized ionic core, the wavefunction is no longer separable in parabolic coordinates and, thus the parabolic quantum number $n_{1}$, which is a good 
quantum number for $\mathrm{H}$, is no longer conserved. As a result, the Stark energy levels exhibit avoided crossings (Fig. 1.4Right).

The up-shifted $(k \gg 0)$ and down-shifted $(k \ll 0)$ states are usually called the blue and red states, respectively. The first energy (avoided-)crossing for states of adjacent $\mathrm{n}$-manifolds (between the bluest $n, m=0$ and reddest $n+1, m=0$ states) occurs at a field [10],

$$
F=\frac{1}{3 n^{5}}
$$

It is instructive to consider the spatial distribution of the wavefunction of hydrogenic Stark states. We have already discussed that for hydrogen and hydrogenic ions, to lowest order in perturbation theory, the wavefunctions do not change with the external electric field and can be described by the zero-field parabolic eigenfunctions. Fig. 1.5 shows the electron charge distribution (square of the wavefunction $\psi^{2}(x, z)$ ) in a plane containing the $z$ axis for hydrogenic Stark states in the $n=10$ manifold. As shown, the blue (red) states are preferentially located along the $+z(-z)$ axes (for a field directed along $+\hat{\boldsymbol{z}}$ ), corresponding to the bounded (unbounded) directions in the presence of an electric field. States near the center of the manifold for a given $n$ (i.e. with $|k| \ll n$ ) exhibit little polarity and are distributed about the $z=0$ plane.

For non-H Rydberg atoms, the fact that $k$ is not a good quantum number indicates that the Stark states are coupled through their slight overlap near the ionic core. Classically, the non-Coulombic part of the potential results in orbits that do not close. Instead, the electron travels a little too fast near the ionic core and, therefore, can scatter from one Stark-trajectory to another each time it passes near the ionic core. Quantum mechanically, this means a blue shifted bound state can be coupled to a red shifted continuum state through the core-scattering. 


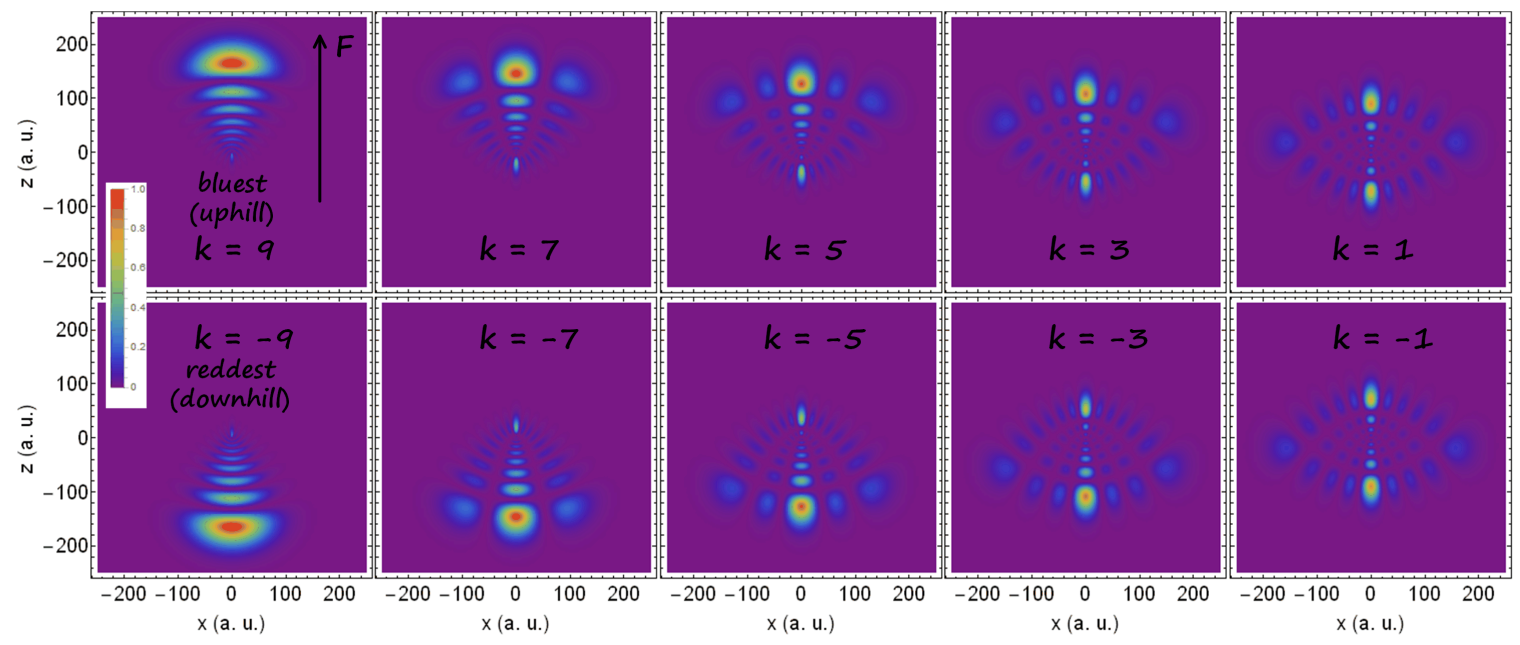

Figure 1.5: Charge distribution for the hydrogenic parabolic eigenstates in a plane containing the $z$ axis: $n=10, m=0, k=n_{1}-n_{2}=-9, \cdots, 9$. $\psi^{2}(x, z) \propto(\xi+\eta) \psi_{n k m}^{2}(\xi, \eta, \phi=0)$. The color represents the charge density in arbitrary units.

\subsection{Strong field physics:}

\section{Ionization and post-ionization dynamics}

\subsubsection{Light induced atomic ionization}

We are familiar with the wave-particle duality of light, accordingly, light induced atomic ionization may be divided into two categories: photoionization and field ionization, as depicted in Fig. 1.6. In photoionization, the incoming light is treated as a flux of photons, and its influence on the atomic structure is small. When the distortion of the atomic potential induced by the light field is too big to be treated as small perturbation, the strong field regime has been reached, and field ionization may occur. 

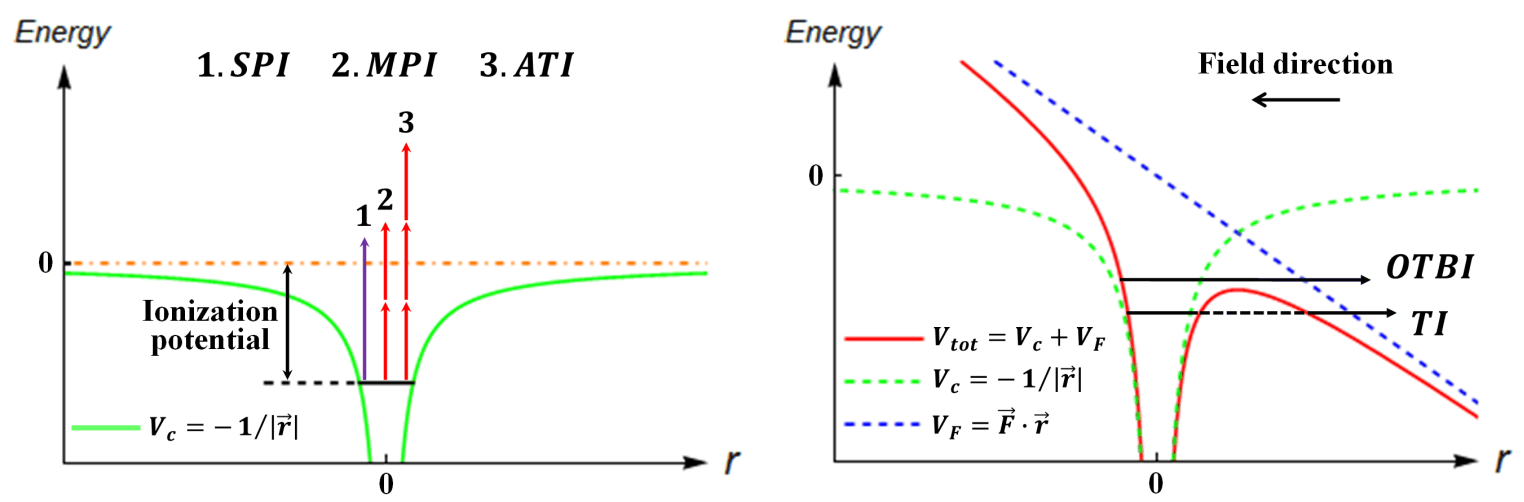

Figure 1.6: Left) Photoionization and Right) Field ionization from a Coulomb potential. SPI: Single-photon ionization; MPI: Multi-photon ionization; ATI: Above threshold ionization; TI: Tunneling ionization; OTBI: Over-the-barrier ionization.

\section{Photoionization}

Photoionization occurs by the absorption of $N(N \geq 1)$ photons by a bound electron which gains an energy of $N \omega$, sufficient to overcome its ionization potential (or binding energy) $I P$. Here $\omega$ is the laser frequency and $N$ is the minimum number of photons required for ionization: $(N-1) \omega<I P \leq N \omega$. At low laser intensities, ionization is possible only if $\omega>I P$, and one photon is absorbed in the process. This is the simplest photoionization scenario: single-photon ionization (SPI). As the laser intensity increases, the probability for the electron to absorb more than one photon and make transitions through virtual or real intermediate states into continuum increases. This is multi-photon ionization (MPI) [14]. Above threshold ionization (ATI) [15] is a special case of MPI where the electron absorbs an extra $S(S \geq 1)$ photons beyond the minimum number $N$ required for ionization. It occurs at rather high laser intensities where the field-distorted atomic potential may influence the photoionizing electron in the continuum, enabling the absorption of extra photons via free-free transitions in the continuum. High order $(S \gg 1)$ ATI is non-perturbative in nature, demonstrating many features of light-matter interactions in the strong field regime. 


\section{Field ionization}

Fig. 1.6Right shows the simplest field-ionization picture, assuming a static or quasistatic field. In the presence of an external electric field $\mathbf{F}=-F \hat{\boldsymbol{z}}$, The combined Coulomb-Stark potential along the $\mathrm{z}$ axis,

$$
V(z)=-\frac{Z}{|z|}-F z
$$

has a saddle point at $z_{s}=\sqrt{Z / F}$ (the saddle point is defined as $\left.\frac{\partial V(z)}{\partial z}\right|_{z_{s}}=0$, and $\mathrm{Z}$ is the effctive ionic core charge). Ionization is classically allowed if the energy of the electron $E=-I P$ lies above the saddle point potential [10],

$$
E \geq V\left(z_{s}\right)=-2 \sqrt{F Z} \quad \text { or } \quad F \geq \frac{E^{2}}{4 Z}=\frac{I P^{2}}{4 Z}
$$

$F=I P^{2} /(4 Z)$ is the classical over-the-barrier ionization (OTBI) threshold. At energies below the saddle point potential, ionization can still occur via quantum tunneling. And there are different models to calculate the tunneling ionization rate. For example, a commonly used model is the Ammosov-Delone-Krainov (ADK) model [16].

\section{Keldysh parameter}

The first attempt to analytically study strong field light-matter interactions was made by Keldysh in 1965 [17]. Keldysh treated multi-photon ionization and tunneling ionization as two limiting cases of the same ionization process, and introduced the tunneling frequency " $\omega_{t}$ ", and a parameter " $\gamma$ ", nowadays known as the Keldysh parameter, to represent the adiabaticity of the process.

$$
\begin{gathered}
\omega_{t}=F / \sqrt{2 I P} \\
\gamma=\frac{\omega}{\omega_{t}}=\frac{\omega \sqrt{2 I P}}{F}
\end{gathered}
$$

Here $\omega$ is the laser frequency, $F$ is the peak laser field strength, and $I P$ is the ionization potential of the bound electron. The Keldysh parameter characterizes the two 
ionization mechanisms by defining the strength and rate of the time varying laser field relative to the atoms under study: tunneling ionization dominates at strong fields and low frequencies $(\gamma \ll 1)$ where the tunneling rate is high, the field change is slow, and the electron has enough time to tunnel out of the field-dressed potential barrier during any half-cycle of the oscillating field. The ionization is adiabatic with no net energy exchange between the field and the electron. Whereas for weak fields and high frequencies $(\gamma \gg 1)$, there is insufficient time for tunneling to occur while the field is large, and ionization can only occur if sufficient energy is transfered to the electron, hence multi-photon ionization dominates.

$$
\gamma \begin{cases}\ll 1, & \text { tunneling ionization } \\ \gg 1, & \text { multi-photon ionization }\end{cases}
$$

Apparently, $\gamma \sim 1$ marks the boundary between the perturbative and strong field regimes.

\section{Ponderomotive energy}

The ponderomotive energy (or ponderomotive potential, $U_{p}$ ), is the cycle-averaged quiver energy of a free electron in an oscillating electric field,

$$
U_{p}=\frac{\left\langle\mathbf{A}^{2}(t)\right\rangle}{2}=\frac{F^{2}}{4 \omega^{2}}
$$

here $\mathbf{A}(t), F$ and $\omega$ are the vector potential, maximum amplitude and central frequency of the laser field, respectively. The quiver amplitude of the electron's motion in the oscillating field is defined as,

$$
l_{q}=\frac{F}{\omega^{2}}
$$

The Keldysh parameter can be rewritten as a function of $U_{p}$ and $I P$,

$$
\gamma=\sqrt{\frac{I P}{2 U_{p}}}
$$


$\gamma$ approaches 1 as $U_{p}$ approaches $I P$, therefore $U_{p} \gg I P$ is an alternative definition of the strong field regime. This is reasonable, because $U_{p}$ is a measure of the electron's kinetic energy in the continuum. An electron which adiabatically ionizes via tunneling or OTBI gains sufficient energy in the continuum to escape the atomic potential.

We have briefly discussed the dependence of the two principal ionization mechanisms on the laser field amplitude (or intensity) and frequency (or photon energy) based on the Keldysh theory. Further, we presented the Keldysh parameter $\gamma$ or the ratio of the ponderomotive energy to the ionization potential, $U_{p} / I P$, as a criterion to determine the "strength" of the laser field relative to the atoms under study. From the expressions of $\gamma$ and $U_{p}$, it is apparent that with low frequency lasers, less intensity is required to enter the strong field regime. Equivalently, for the same intensity, low frequency fields can transfer more energy to a free electron, as the electron "quivers" and is accelerated over a longer distance/time. Accordingly, it can be advantageous to explore strong field physics with very low frequency radiation, e.g., in the mid-infrared, $\mathrm{THz}$ or even microwave regimes.

Fig. 1.7 is a schematic of different ionization regimes, as a function of scaled laser field strength and photon energy. Tab. 1.2 gives values of relevant parameters for the ionization of ground state hydrogen with extreme-ultraviolet (XUV), near-infrared (NIR) or $\mathrm{THz}$ radiation. 


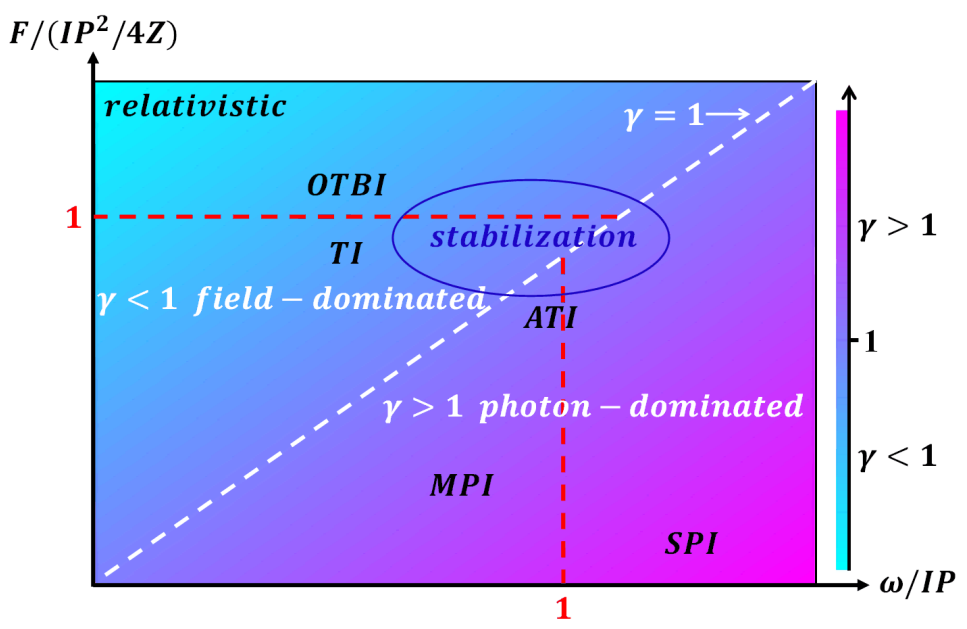

Figure 1.7: Schematic of different ionization regimes based on the Keldysh theory, coordinated by the scaled laser field strength $F^{\prime}=\frac{F}{I P^{2} / 4 Z}$ and photon energy $\omega^{\prime}=\frac{\omega}{I P}$. The color represents the value of Keldysh parameter as a function of $F^{\prime}$ and $\omega^{\prime}, \gamma=\gamma\left(F^{\prime}, \omega^{\prime}\right)$ on $\log$-log scale. As shown, $\gamma=1$ distinguishes the photon-dominated and field-dominated regimes. In the photon-dominated regime, $\omega=I P$ separates SPI and MPI, whereas ATI occurs as $U_{p}$ approaches the photon energy $\omega$. Strong field features appear at $\gamma \approx 1$. In the field-dominated regime, $F=I P^{2} /(4 Z)$ separates TI and OTBI. Modern laser technologies already enables laser intensities that can accelerate the electron to velocities on the order of the speed of light $c$. This opens the door to the relativistic regime of strong field physics, where the quiver energy of the electron $F^{2} / \omega^{2}\left(4 U_{p}\right)$ is on the order of the electron rest energy $m_{e} c^{2} \simeq 0.51 \mathrm{MeV}$. Under certain conditions, stabilization of ionization (a saturation at less than 1, or a suppression, in ionization probabilities as the laser intensity increases) can be obtained. This is predicted for interactions with intense and high frequency lasers. (See the review article [18], and references therein, for laser-atom interactions covering the regimes mentioned above.)

Table 1.2: Values of relevant parameters for the ionization of ground state hydrogen $\left(I P=13.6 \mathrm{eV}, I P^{2} / 4=3.21 \mathrm{E} 5 \mathrm{KV} / \mathrm{cm}\right)$ with XUV, NIR or $\mathrm{THz}$ radiation. The units for field strength $F$, intensity $I$ and quiver amplitude $l_{q}$ are $\mathrm{kV} / \mathrm{cm}, \mathrm{W} / \mathrm{cm}^{2}$ and $\mathrm{nm}$, respectively.

\begin{tabular}{c|ccc|ccc|ccc}
\hline & \multicolumn{3}{|c|}{ XUV $(80 \mathrm{~nm}, 15.5 \mathrm{eV})$} & \multicolumn{3}{c|}{ NIR $(800 \mathrm{~nm}, 1.55 \mathrm{eV})$} & \multicolumn{4}{c}{ THz $(0.8 \mathrm{~mm}, 1.5 \mathrm{meV})$} \\
\hline \hline$N[\omega / I P]$ & \multicolumn{3}{|c|}{1} & \multicolumn{3}{c}{9775} \\
$\gamma\left(F=I P^{2} / 4\right)$ & \multicolumn{3}{|c|}{9.12} & \multicolumn{3}{c}{0.912} & \multicolumn{4}{c}{$9.12 \mathrm{E}-4$} & \\
$F, I, l_{q}\left(U_{p}=\omega\right)$ & $4.42 \mathrm{E} 6$ & $2.59 \mathrm{E} 16$ & $1.40 \mathrm{E}-1$ & $1.40 \mathrm{E} 5$ & $2.59 \mathrm{E} 13$ & $4.43 \mathrm{E}-1$ & $4.42 \mathrm{E} 0$ & $2.59 \mathrm{E} 4$ & $1.40 \mathrm{E} 1$ \\
$F, I, l_{q}(\gamma=1)$ & $2.93 \mathrm{E} 6$ & $1.14 \mathrm{E} 16$ & $9.29 \mathrm{E}-2$ & $2.93 \mathrm{E} 5$ & $1.14 \mathrm{E} 14$ & $9.29 \mathrm{E}-1$ & $2.93 \mathrm{E} 2$ & $1.14 \mathrm{E} 8$ & $9.29 \mathrm{E} 2$ \\
$F, I, l_{q}\left(U_{p}=\frac{m_{e} c^{2}}{4}\right)$ & $4.01 \mathrm{E} 8$ & $2.14 \mathrm{E} 20$ & $1.27 \mathrm{E} 1$ & $4.01 \mathrm{E} 7$ & $2.14 \mathrm{E} 18$ & $1.27 \mathrm{E} 2$ & $4.01 \mathrm{E} 4$ & $2.14 \mathrm{E} 12$ & $1.27 \mathrm{E} 5$ \\
\hline \hline
\end{tabular}




\section{Field ionization by pulsed and oscillating fields}

In the Keldysh theory, which is based on a zero range potential, the laser period (or frequency) is compared with the atomic tunneling ionization time (or frequency). For Rydberg atoms with spatially extended binding potentials, another important time scale is the Kepler orbital period of the electron. The ratio of the laser frequency $\omega$ to the electron's Kepler orbital frequency $\omega_{k}$,

$$
\Omega=\frac{\omega}{\omega_{k}}=\frac{T_{k}}{T}=\omega n^{3}
$$

a.k.a. the scaled frequency, determines how far the electron moves in one field cycle, and how many times it "visits" the ionic core in that period. Because of the extended orbit size and the availability of orbitals with a wide range of angular momenta, the ionization dynamics are far more rich than those associated with classical over the barrier or tunneling ionization in ground state systems. An introduction to Rydberg field ionization mechanisms in pulsed and oscillating fields is included in Chap. 3.

\subsubsection{Post-ionization dynamics}

\section{Three-step model}

A strong, oscillating electric field may not only induce field ionization, but also influences the liberated electron. The process has been described by the three-step model [19-21]. The standard three-step model involves (see Fig. 1.8): 1) Ionization: The electron is liberated from the trap of the atomic potential through, for example, tunneling ionization. 2) Propagation: At first, the liberated electron is accelerated away from the ionic core by the ionizing field. As the direction of the field changes, so does the direction of the acceleration, and the electron may be driven back to

the ionic core. The propagation in the continuum, as well as the momentum and energy transfer from the field to the electron during propagation, are described clas- 
sically by the so called "simpleman's model" [22-24]. 3) Rescattering: The electron re-scatters with the ionic core, upon which various phenomena may occur, including 3a) recombination via photon emission, i.e., high harmonic generation (HHG), 3b) elastic scattering resulting in the ejection of the energetic rescattered electron, and 3c) inelastic scattering yielding multi-electron emission, and/or core excitation.
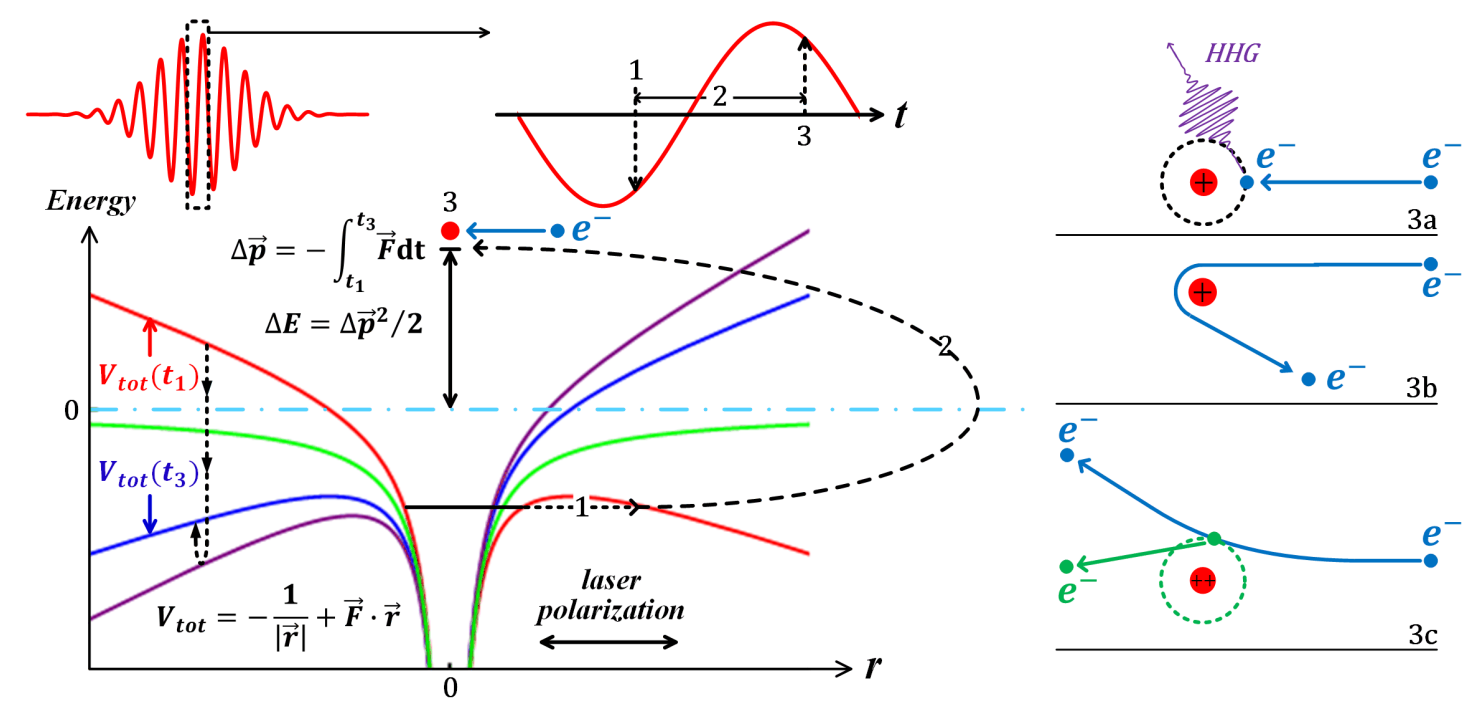

Figure 1.8: The three-step model.

\section{Kinematics of rescattering [25]}

If an electron is "born" at rest at time $t_{0}$ in an electric field with vector potential $\mathbf{A}(t)$, its velocity is described by,

$$
\mathbf{v}(t)=\mathbf{p}+\mathbf{A}(t)
$$

here $\mathbf{p}=-\mathbf{A}\left(t_{0}\right)$ is the conserved canonical momentum, a.k.a., the drift momentum in the field. The electron trajectory can be found by integrating the velocity,

$$
\mathbf{r}(t)=\int_{t_{0}}^{t} \mathbf{v}\left(t^{\prime}\right) \mathrm{d} t^{\prime}=\int_{t_{0}}^{t} \mathbf{A}\left(t^{\prime}\right) \mathrm{d} t^{\prime}-\mathbf{A}\left(t_{0}\right)\left(t-t_{0}\right)
$$

The phase of the electric field at which the electron is "born" in the continuum 
determines whether the electron can return to the ionic core at a later time or not, as well as the momentum and energy transfer from the field to the electron (Fig. 1.9). For a many-cycle laser pulse in the "short pulse" regime, where the laser field turns off before the electron has a chance to leave the laser focus, the direct electrons (electrons that never return to the ionic core), returned electrons, and backscattered electrons (electrons that experience elastic scattering at $180^{\circ}$ ), can obtain maximum kinetic energies,

$$
E_{d}\left(t_{\infty}\right)=2 U_{p} \quad, \quad E_{r}\left(t_{\text {return }}\right)=3.17 U_{p} \quad, \quad E_{b s}\left(t_{\infty}\right)=10 U_{p}
$$
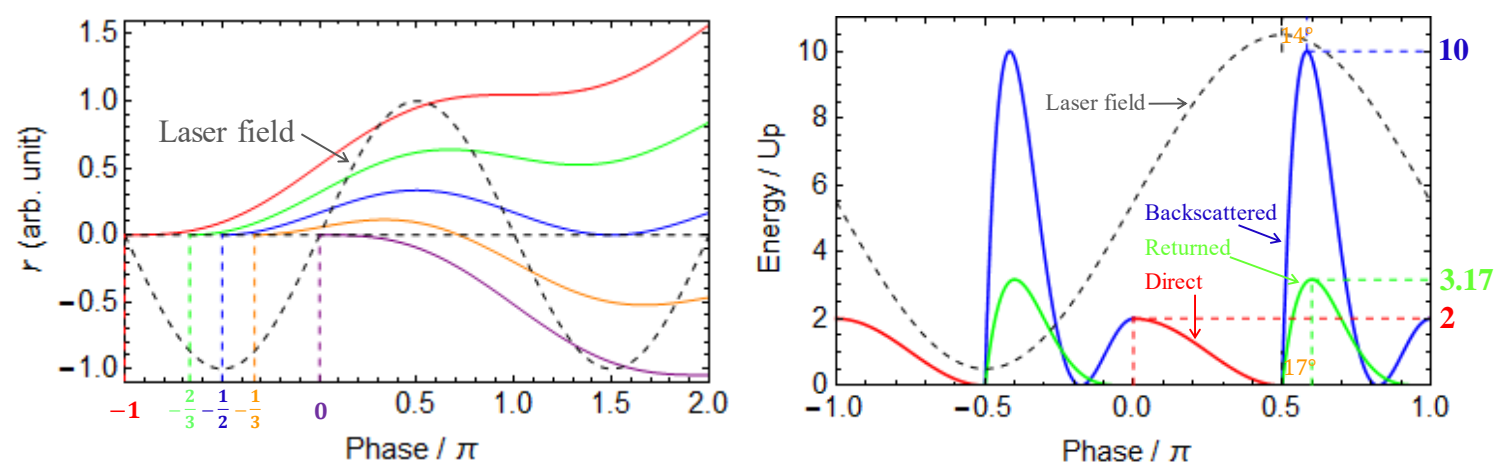

Figure 1.9: Illustration of the phase dependence in the rescattering process, considering a linearly polarized laser field with sinusoidal waveform $F=F_{0} \sin \omega t$. Left) Trajectories of electrons "born" at several different phases of the laser field (The effects of the electron-core collision are not shown). Electrons born early in any half cycle never return to the ionic core, whereas electrons born later in the half cycle may return and collide with the ionic core. Right) Energies for direct, returned, and backscattered electrons as a function of their "birth" phase, assuming one rescattering event.

Details of the rescattering kinematics can be found in Appendix C. To emphasis, the situation needs to be reconsidered in the case of a single-cycle field, as will be described in detail in Chap. 3: High-energy rescattered electrons are not produced in atomic ionization induced by single-cycle pulses. However, enormous energy transfer far exceeding $2 U_{p}$ from the field to the electron can occur without backscattering. 


\subsubsection{Strong field physics in nanostructured solids}

The study of the "ionization" of solid state systems has a long history, dating back to the 1900s. Hertz discovered the photoelectric effect in 1887 [26] and in 1905 Einstein's theoretical explanation [27] gave the first picture of how electrons escape the attraction of a metal by absorbing the energy of discrete photons to overcome the work function. In contrast to this "photo-emission" process, electrons may also be extracted from solids via quantum tunneling in the presence of a strong electric field, analogous to atomic tunneling ionization. Such process is known as field emission and the theory was first considered in detail by Fowler and Nordheim in 1928 [28]. In field emission, an extremely high electric field, typically over $10 \mathrm{MV} / \mathrm{cm}$, is required to sufficiently depress the solid-vacuum potential barrier and produce measurable tunneling emission current. In 1935, Müller produced such a high electric field by making use of the strong field enhancement in the vicinity of sharp structures [29], this laid the groundwork for electron-based microscopy.
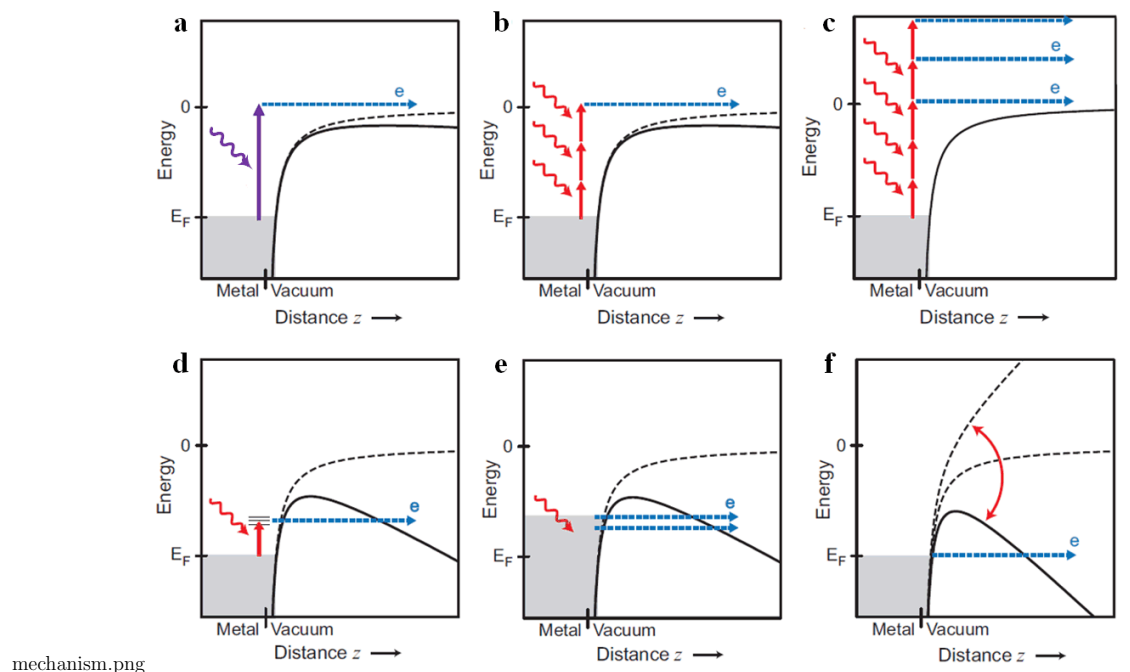

Figure 1.10: Optical liberation of electrons from a metal, from Ref. [30]. a) single-photon photo-emission (the original photoelectric effect), b) multi-photon photo-emission, c) above-threshold photo-emission, d) photon-assisted field-emission (photo-field emission), e) thermo-enhanced field-emission, f) optical field-emission. 
Compared to atomic and molecular gases, spectroscopy of solids is more complex, mainly because many body interaction in condensed systems complicate the underlying physics. In addition, the damage thresholds of bulk materials limit the highest laser intensity that can be applied for the exploration of strong field physics.

During the last decade, numerous studies have explored strong-field physics in solids by coupling femtosecond laser pulses to nanostructured solids, particularly metallic nano-tips. The attraction of nanostructures lies in the following two features: First, the large field enhancement. Not only the enhancing mechanism is of great interest, for example, we can study the plasmonic response of nanostructures to a field, but also the enhanced field helps access the strong field regime. Second, the spatial localization of the enhanced field potentially can generate strong-field dynamics exclusive to nanostructures. The results of those investigations on femtosecond laser induced electron emission from nanostructured solids demonstrated that, with proper modification, much of the basic physics underlying laser induced ionization of atomic and molecular gases also applies to laser-induced electron emission from solids.

More detail about recent developments in this area will be introduced in Chap. 5 . For now, let us review some basic principles.

\section{Fowler-Nordheim equation}

The tunneling emission current density $J$ of field emission from a cold metal (cold field emission (CFE)) is given by [31, 32],

$$
J=\lambda_{T} \lambda_{B} \lambda_{P} P_{F} \tau_{F}^{-2} \frac{a F^{2}}{\Phi} \exp \left(-\nu_{F} \frac{b \Phi^{3 / 2}}{|F|}\right)
$$

Here $F$ is the laser field strength and $\Phi$ is the metal work function; $a=e^{3} /\left(16 \pi^{2} \hbar\right)$ and $b=4 \sqrt{2 m_{e}} /(3 \hbar e)$ are the first and second FN constants, respectively; $\tau_{F}$ and $\nu_{F}$ are slowly varying functions of $F, P_{F}$ is the tunneling prefactor; $\lambda_{T}, \lambda_{B}$, and $\lambda_{P}$ are 
correction factors due to temperature, electron band-structure and the variation of $P_{F}$ with barrier height, respectively. In the standard Fowler-Nordheim-type tunneling, assuming a triangular barrier rounded by the image-potential of the tunneling electron (Schottky-Nordheim barrier), $P_{F}, \lambda_{T}, \lambda_{B}$, and $\lambda_{P}$ are all close to unity, and Eq. 1.29 can be simplified to [33],

$$
J=t^{-2}(f) \frac{a F^{2}}{\Phi} \exp \left(-v(f) \frac{b \Phi^{3 / 2}}{|F|}\right)
$$

where,

$$
\begin{gathered}
f=\left(e^{3} / 4 \pi \epsilon_{0}\right)|F| / \Phi^{2} \\
v(f) \approx 1-f+\frac{1}{6} f \ln f \approx(0.4 \sim 0.8) \\
t(f)=v(f)-\frac{4}{3} f \frac{\mathrm{d} v(f)}{\mathrm{d} f} \approx 1
\end{gathered}
$$

Alternatively, the Fowler-Nordheim relation can be expressed as,

$$
\ln \left(J / F^{2}\right)=C_{1} \frac{1}{F}+C_{2}
$$

here $C_{1}$ and $C_{2}$ are (approximately) constants. Eq. 1.32 explicitly shows that the plot of $\ln \left(J / F^{2}\right)$ versus $1 / \mathrm{F}$ is a straight line.

\section{Field enhancement}

Field enhancement may be induced by plasmonic resonances, antenna resonance or

the geometric lightning-rod effect. The $\mathrm{THz}$ field enhancement in the vicinity of tungsten nano-tips arises from the lightning-rod effect.

To study near-field electron dynamics in the vicinity of a nanostructure, the spatial distribution of the field near the nanostructure needs to be known. Typically, numerical calculation of the field distribution involves finite-difference-time-domain (FDTD) methods. Such calculations are difficult and and require knowledge of the applied field, the shape of the structure, and its electric proprieties. However, in some 
cases, an analytical approach is possible. For example, upon applying a DC field to a hyperboloidal shaped tip, the field along the tip axis (defined as the $z$-axis) is given by [34],

$$
F(z \geq 0)=\frac{F_{0}}{1+2 z / r_{0}}
$$

here $z$ denotes the distance from the tip apex. Eq. 1.33 shows that the field decays rapidly along the $z$-axis.

For hemisphere-capped cylinder tip emitters (see Fig. 1.11), the DC field enhancement factor $\gamma$ has an almost linear dependence on $L / R$,

$$
\gamma=A+B\left(\frac{L}{R}\right)^{C}
$$

where $L$ and $R$ are the tip length and radius, respectively. A, B and C are parameters that are close to 1. The exact expression is more complex. See Ref. [35] and references therein for more detail.

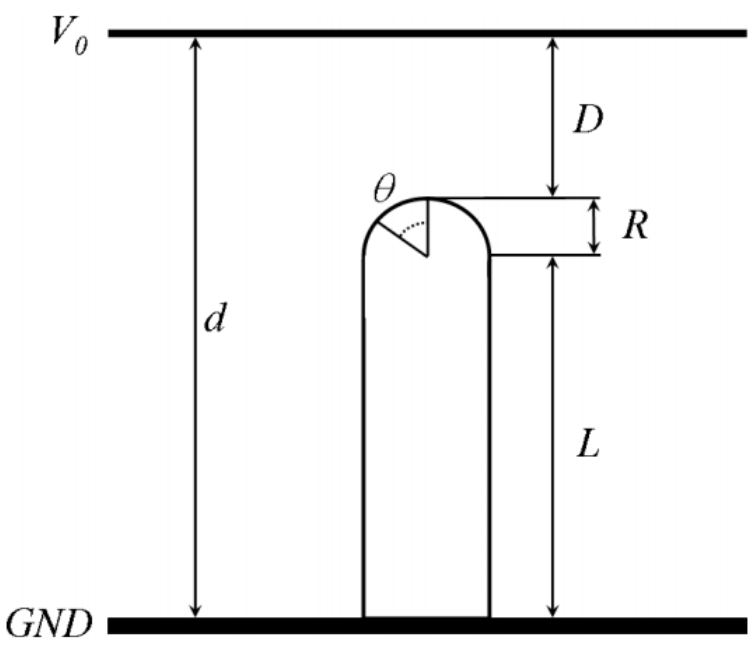

Figure 1.11: Geometry of a hemisphere capped cylindrical field emitter, from Ref. [35]. 


\section{$1.4 \mathrm{THz}$ radiation}

$\mathrm{THz}$ radiation, sometimes classified as sub-millimeter or far-infrared (FIR) radiation, has a frequency/wavelength that falls in between that of infrared and microwave radiations $[36,37]$. The $\mathrm{THz}$ frequency band does not have a strict definition, but is generally considered to cover a range from 0.1 to $10 \mathrm{THz}$ (periods $10-0.1 \mathrm{ps}$, wavelengths 3-0.03 mm, wavenumbers $3.33-333 \mathrm{~cm}^{-1}$, photon energies $\left.0.414-41.4 \mathrm{meV}\right)$. Due to the lack of efficient and compact $\mathrm{THz}$ sources and sensors, $\mathrm{THz}$ radiation is not as well explored as radiations at other frequencies in the electromagnetic spectrum, and has long been described as the "THz gap". It was not until the demonstration of reliable $\mathrm{THz}$ generation and detection in the 1980s and early 1990s [38-47] that $\mathrm{THz}$ research began to rapidly develop.

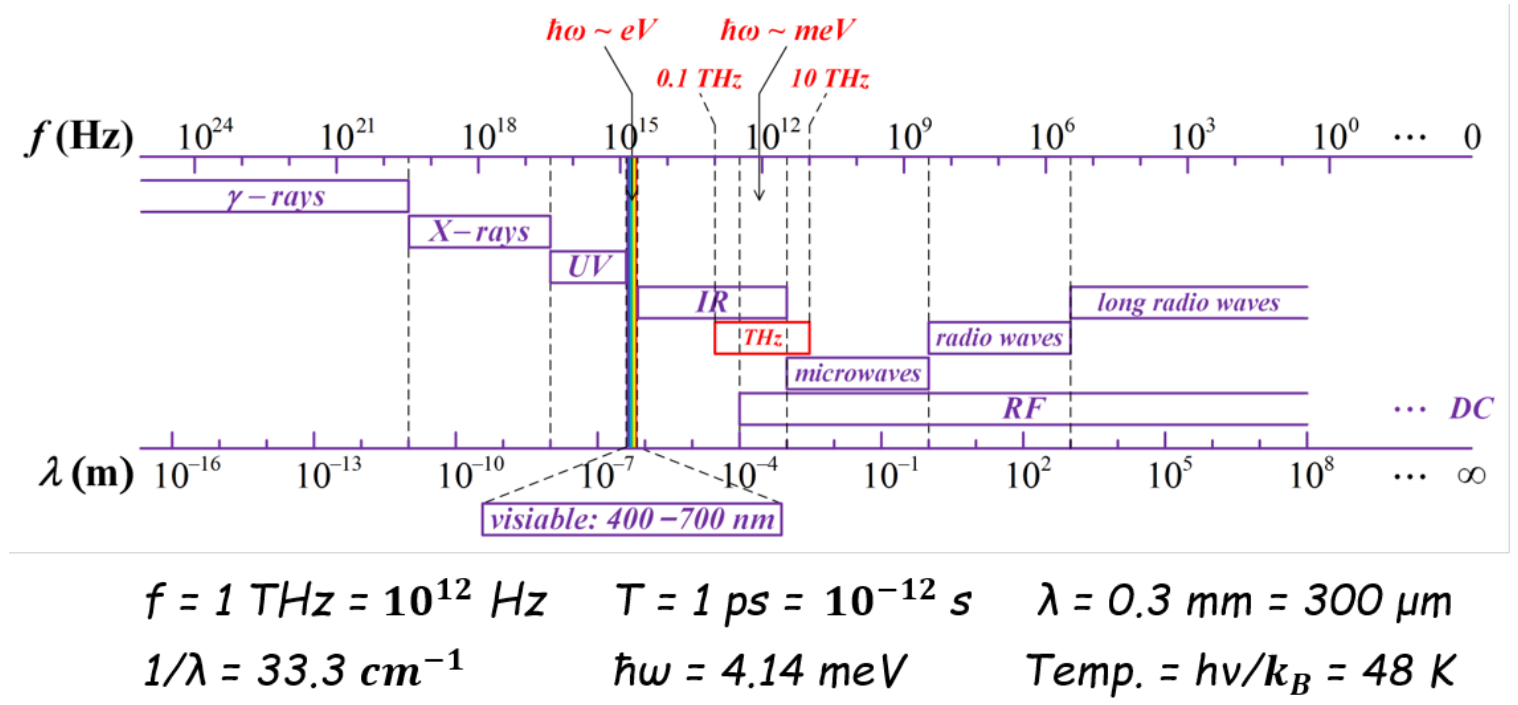

Figure 1.12: Electromagnetic spectrum and characteristics of $f=1 \mathbf{T H z}$ radiation.

Characteristics: Fig. 1.12 shows a typical classification of different types of radiation as they appear in the spectrum of electromagnetic waves, and the characteristics of $f=1 \mathrm{THz}$ radiation. One important feature of $\mathrm{THz}$ radiation is that in contrast 
to optical waves, it has low frequency and low photon energy. Also, the response of condensed matter systems to $\mathrm{THz}$ radiation varies widely. For example, water and other strongly polar liquids are highly absorptive in the $\mathrm{THz}$ frequency regime; metals are highly reflective at $\mathrm{THz}$ frequencies due to high electrical conductivity; dielectrics, such as paper, plastic, clothes, woods, ceramics etc., are highly transparent to $\mathrm{THz}$ radiation [36].

Generation and detection: There exist many methods of generating $\mathrm{CW}$ or pulsed THz radiation (for example, see review books $[36,37]$ and review articles $[48$, 49] and references therein). Depending on the generation mechanism, these methods can be classified as: a) laser emission through population inversion (Fig. 1.13A), b) frequency conversion in nonlinear materials (Fig. 1.13B), and c) radiation by charge acceleration or photocurrent variation (Fig. 1.13C). Among these methods, optical rectification in electro-optic crystals, photocarrier acceleration in photoconductive antennas and electron acceleration in laser induced gas plasmas (four-wave mixing enhanced) are widely applied in laboratory-based time-domain THz research today. All three of these involve the use of ultrafast femtosecond lasers.

Detection of pulsed $\mathrm{THz}$ radiation typically involves either coherent field-amplitude and -phase measurements, which can be realized by making use of the nonlinear fieldresponse of an electro-optic crystal or a photoconductive antenna or mixer to combine femtosecond-optical and THz fields (Fig. 1.14), or incoherent pulse fluence measurements with, for example, thermal sensors such as pyroelectric detectors or bolometers.

Fundamental applications: In contrast to optical waves, $\mathrm{THz}$ radiation allows direct access to numerous fundamental processes that resonate at low frequencies, such as coherent transitions between Rydberg levels, rotational transitions of molecules, large-amplitude vibrational motions of organic compounds, intra-band transitions of semiconductors, spin control of (anti-)ferromagnets, etc. Strong THz 
fields can also induce non-resonant light-matter interactions, for example, field ionization of atomic and molecular gases, field emission from solids, manipulation of high-energy electron trajectories, nonlinear modulation of the optical properties of solids, etc. Reviews of the applications of $\mathrm{THz}$ radiation in fundamental research can be found in Ref. [36, 37, 50] and references therein.

Practical applications: The characteristics of $\mathrm{THz}$ radiation, including low photon energy (which is non-ionizing), low-frequency (that gives resonant spectral fingerprints to molecules) and high transparency to many materials, open the door to practical applications of $\mathrm{THz}$ radiation in non-destructive spectroscopy, microscopy, and imaging. The use of $\mathrm{THz}$ radiation for quality evaluation, security scanning, biomedical imaging, etc., has been demonstrated. Ultrafast wireless communication in the $\mathrm{THz}$ frequency band is also very promising. See Ref. [36, 37, 51] and references therein for practical applications of $\mathrm{THz}$ radiation. 


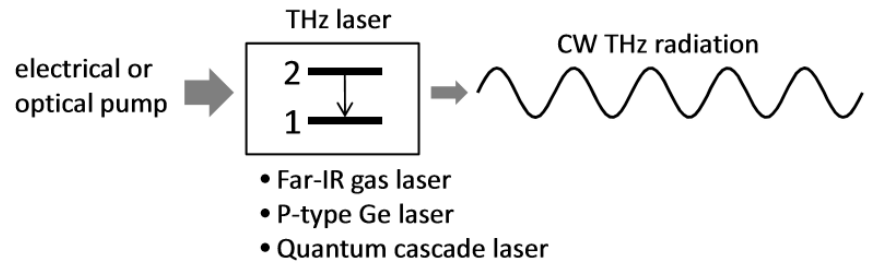

\section{A. THz emission from lasers}

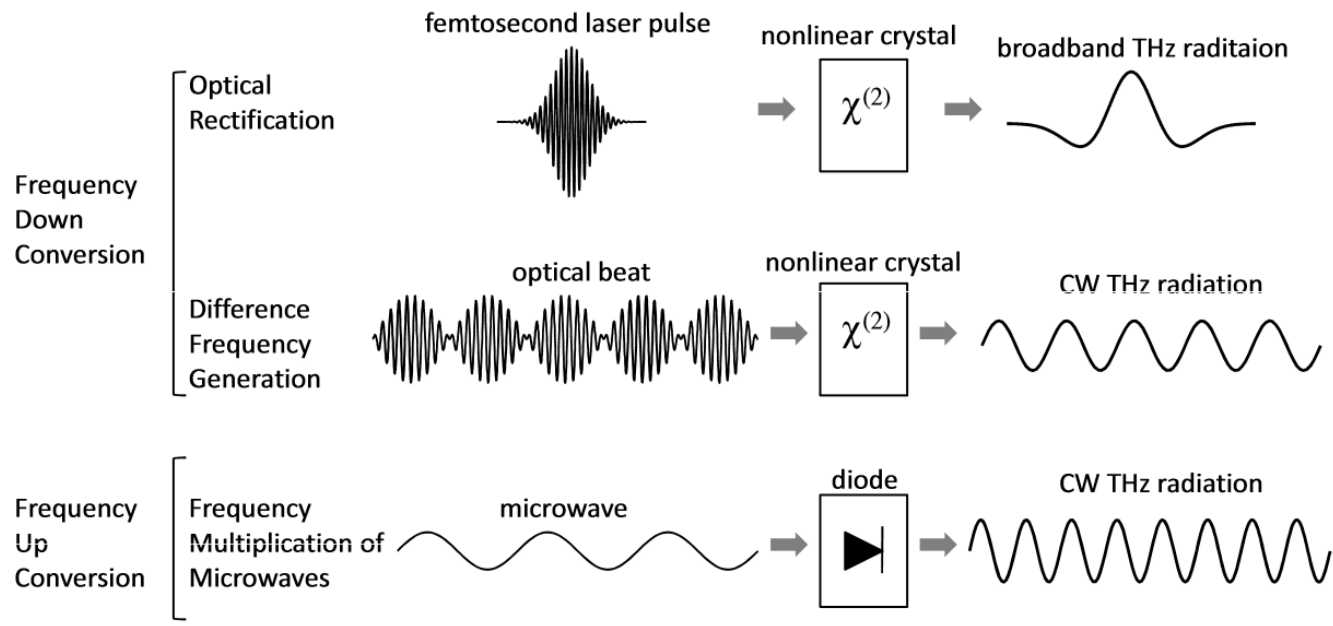

\section{B. THz generation in nonlinear media}

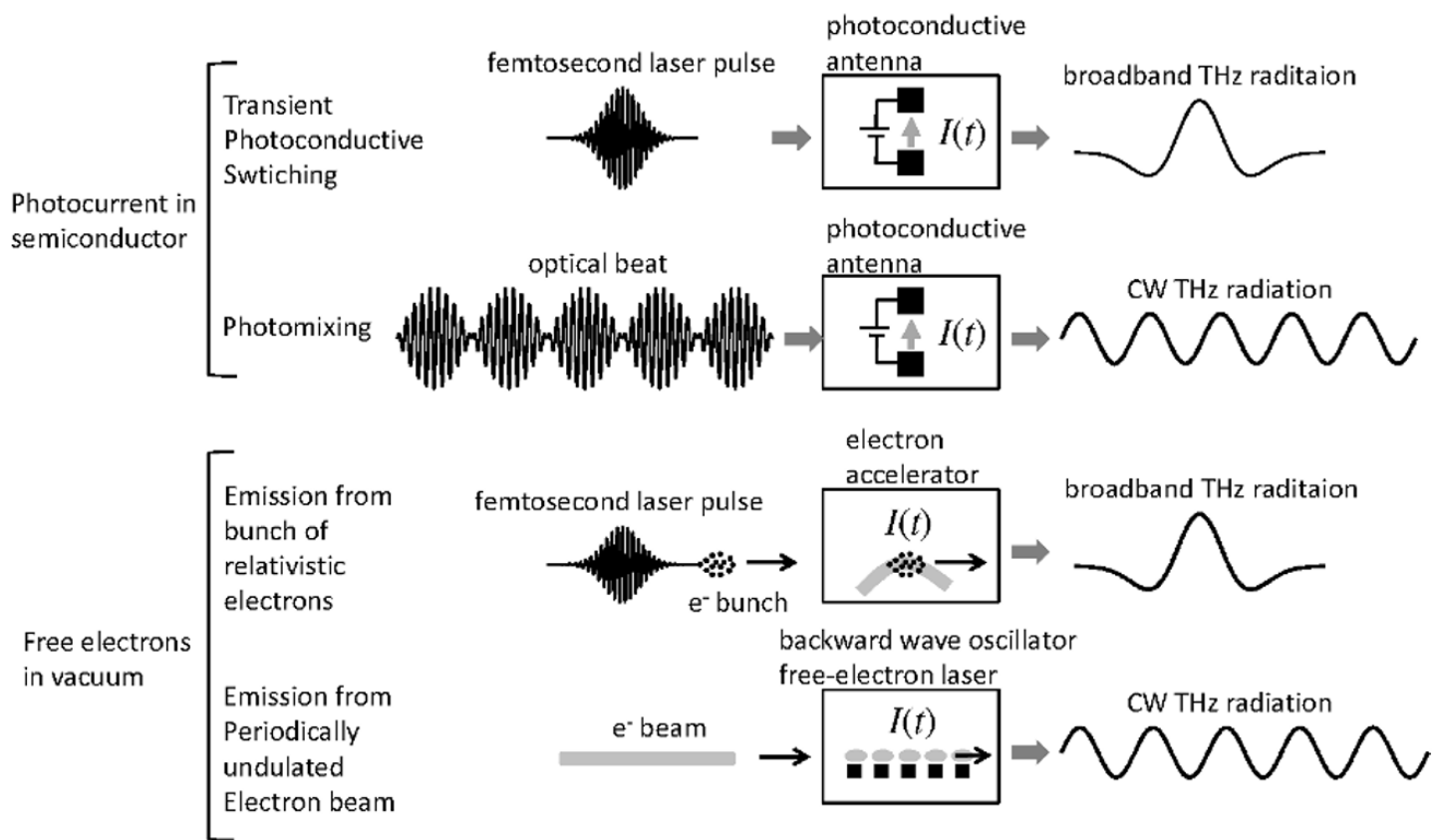

\section{THz radiation from accelerating electrons}

Figure 1.13: Methods of generating $\mathrm{THz}$ radiation. (from Ref. [36]) 

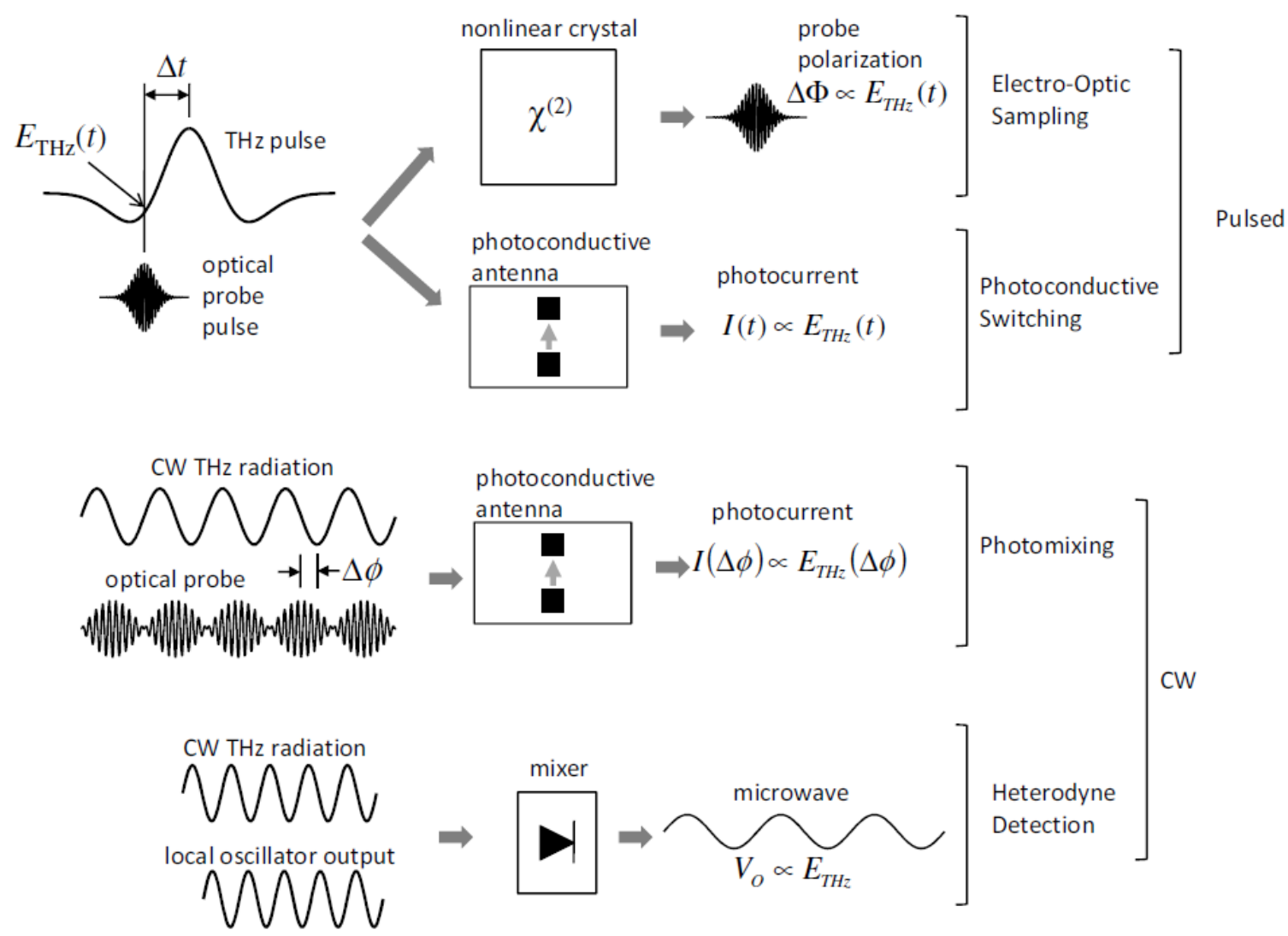

Figure 1.14: Coherent detection of $\mathrm{THz}$ radiation. (from Ref. [36]) 


\section{Chapter 2}

\section{Experimental setups and concepts}

\subsection{Overview}

This chapter introduces experimental setups and methods, as well as related concepts and techniques, for work done in this dissertation. At a glance, the experimental realization involves the following apparatus and tools:

- Optical system

Femtosecond Ti:sapphire oscillator-amplifier; THz source; Hänsch/Littman dye lasers.

\section{- High vacuum system}

The vacuum chamber is two-stage pumped (diffusion pump + mechanical pump), with a base pressure as low as $\sim(2-3) \times 10^{-7}$ Torr. Liquid nitrogen cold traps can be used to further lower the pressure to $\sim 1 \times 10^{-7}$ Torr.

- Samples

Thermal atomic beam of $\mathrm{Na}$ is produced by resistively heating a Na oven. Tungsten nanotips are prepared via electro-chemical etching.

\section{- Detector}


The charged particle detector is a double-chevron-stacked micro channel plate (MCP) detector that outputs a pulsed voltage signal which is proportional to the incident electron/ion flux. In the experiments, the MCP detector functions as an electron/ion sensor, part of a time-of-flight electron spectrometer, or part of a retarding potential energy analyzer.

\section{- Data acquisition and analysis}

LeCroy 9350A (500 MHz), Tektronix 2440/2430 (500/200 MHz) oscilloscopes; Computers with custom data collection software; Analyzing/Simulation software (Origin, MATLAB, Mathematica, etc.).

\section{- Timing}

Lasers run at a $15 \mathrm{~Hz}$ repetition rate. Relative timing is controlled by several Stanford Research DG535 delay generators.

\section{$2.2 \quad$ Femtosecond laser system}

Ever since the discovery of Kerr lens self mode-locking (KLM) in Ti:sapphire lasers in 1991 [52], rapid progress has been made in the generation of ultrashort femtosecond pulses with solid state lasers. Nowadays, Ti:sapphire lasers are capable of directly generating sub-5 fs, sub-2 optical cycle pulses $[53,54]$. Typically a Ti:sapphire oscillator is pumped by a continuous-wave $(\mathrm{CW})$ laser, and delivers pulses with several nanojoules of energy at 60-200 MHz repetition (rep) rate when mode-locked (the rep rate $f_{\text {rep }}$ is determined by the cavity length $L, f_{\text {rep }}=2 L / c$ ). Microjoule to joule level pulses can be obtained via chirped pulse amplification [55].

The femtosecond laser system used for experiments in this dissertation produces $150 \mathrm{fs}, 790 \mathrm{~nm}$ pulses at a $15 \mathrm{~Hz}$ rep rate, with an average power up to $350 \mathrm{~mW}$, corresponding to a pulse energy up to $23 \mathrm{~mJ}$. It consists of a commercial Ti:sapphire 
oscillator (KMLabs MTS Mini Ti:Sapphire Laser) pumped by a frequency doubled (532 nm) Nd:YVO 4 CW laser (Spectra-Physics Millennia Vs), and a self-built chirped pulse amplifier (CPA) pumped by a $15 \mathrm{~Hz}$, Q-switched, frequency doubled (532 nm) Nd:YAG laser (Spectra-Physics Quanta Ray GCR-100). The CPA uses a two stage design: a regenerative (regen) pre-amplifier followed by a three-pass linear amplifier.

The Ti:sapphire oscillator-amplifier design uses very well developed techniques and details can be found in many places. Sketches of the system I used can be found in Appendix D, as well as in the $\mathrm{PhD}$ theses of former lab members (for example, see $[56,57])$.

\section{3 $\mathrm{THz}$ generation and characterization}

The THz radiation used in the experiments is generated via tilted-pulse-front-pumping (TPFP) optical rectification of femtosecond laser pulses in $\mathrm{LiNbO}_{3}$. The energy and spatial profile of the $\mathrm{THz}$ radiation are measured with a pyroelectric detector; the temporal profile is obtained by electro-optic sampling, as well as photoelectron streaking. As will be described in the experimental section of Chapter 3, another way of characterizing the $\mathrm{THz}$ field strength is via $\mathrm{THz}$ induced field ionization of Rydberg atoms.

\subsubsection{Optical rectification}

Optical rectification is a second order nonlinear process. For a CW laser field (single frequency), it can be considered as the special case of difference frequency generation (DFG) where the two frequencies are identical, and a DC polarization is induced due to this process. For an ultrafast laser pulse (broad bandwidth) of frequency $\omega$ and bandwidth $\Delta \omega$, optical rectification is a intrapulse DFG process, and the induced 
radiation (of frequency $\Omega$ ) is the result of weighted sum of DFG processes between frequency components, $\omega_{1}$ and $\omega_{2}$, contained within the ultrafast pulse bandwidth,

$$
\Omega=\omega_{1}-\omega_{2}
$$

The frequency bandwidth of a femtosecond pulse lies in the THz regime (e.g. for a sinusoidal pulse with Gaussian envelope, $\Delta \tau_{F W H M}=100$ fs corresponds to $\Delta f_{F W H M}=$ $4.41 \mathrm{THz}$ ), thus optical rectification of ultrafast femtosecond laser pulses generates $\mathrm{THz}$ radiation. This process can also be viewed in the time domain: Optical rectification induces a polarization that follows the intensity envelope of the pump field, and the induced polarization is a source of electromagnetic radiation. Thus a pulsed pump will induce a pulsed field with frequency determined by the bandwidth of the pump pulse.

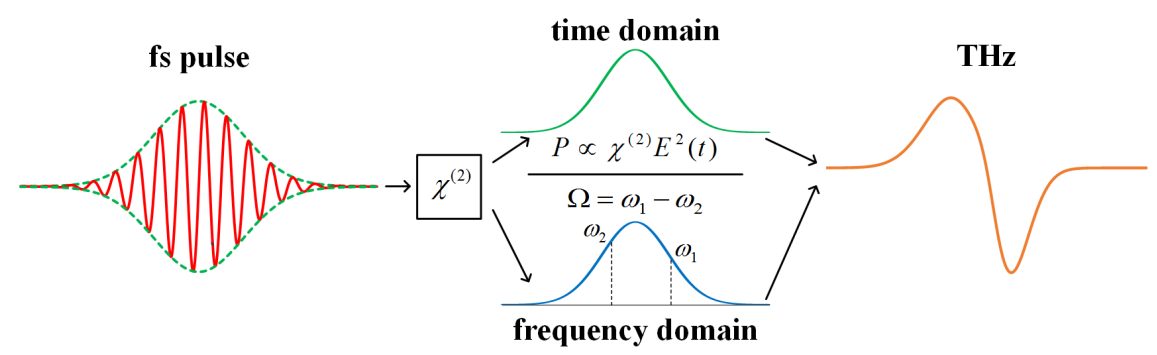

\section{Figure 2.1: Principle of $\mathrm{THz}$ generation via optical rectification.}

For the generation of $\mathrm{THz}$ radiation by optical rectification of fs laser pulses, the conversion efficiency could far exceed the Manley-Rowe limit because cascaded $\chi^{2}$ process is possible [58]. "Cascade" here means that the frequency down conversion continues as long as the phase matching is satisfied so that a pump photon of frequency $\omega$ can be converted into multiple photons of frequency $\Omega$,

$$
\omega \rightarrow\left\{\begin{array}{l}
\omega-\Omega \rightarrow\left\{\begin{array}{l}
\omega-2 \Omega \rightarrow \cdots \\
\Omega
\end{array}\right. \\
\Omega
\end{array}\right.
$$


Efficient nonlinear conversion requires phase matching. In the case of $\mathrm{THz}$ generation by optical rectification of femtosecond laser pulses, it requires,

$$
\left\{\begin{array}{l}
\mathbf{k}_{N I R+T H z}-\mathbf{k}_{N I R}=\mathbf{k}_{T H z} \\
\omega_{N I R+T H z}-\omega_{N I R}=\Omega_{T H z}
\end{array}\right.
$$

In the collinear case, since $\Omega_{T H z} \ll \omega_{N I R}$, dividing the first equation by the second gives,

$$
\left.\frac{\partial k}{\partial \omega}\right|_{\omega_{N I R}}=\frac{k_{T H z}}{\Omega_{T H z}}
$$

Because $\left.v_{N I R}^{g r} \triangleq \frac{\partial \omega}{\partial k}\right|_{k_{N I R}}$ and $v_{T H z} \triangleq \frac{\Omega_{T H z}}{k_{T H z}}$, phase matching is then equivalent to velocity matching, i.e. the group velocity of the NIR pump pulse must equal the phase velocity of the $\mathrm{THz}$ radiation inside the nonlinear material [59],

$$
v_{N I R}^{g r}=v_{T H z} \quad \text { or } \quad n_{N I R}^{g r}=n_{T H z}
$$

Stochiometric $\mathrm{LiNbO}_{3}(\mathrm{sLN})$ is a great candidate for the generation of $\mathrm{THz}$ via optical rectification, mainly because of its relatively high $\chi^{(2)}$ nonlinearity and wide bandgap (wide bandgap reduces multiphoton absorption of the pump pulse which generates free carriers that causes $\mathrm{THz}$ absorption) [59]. But the large index difference between optical and $\mathrm{THz}$ frequencies in $\operatorname{sLN}\left(n_{800 \mathrm{~nm}}^{g r}=2.25, n_{1.55 \mu m}^{g r}=2.18\right.$, $\left.n_{T H z}=4.96\right)$ [59] makes collinear phase matching impossible. Therefore a noncollinear Cherenkov geometry [40,60, 61] or quasi-phase-matching in periodically poled $\mathrm{LiNbO}_{3}$ (PPLN) [62-64] were preferred methods. However, in 2002, Hebling et al. proposed a tilted-pulse-front-pumping scheme for phase matching [65]. This technique has been rapidly developed over the last 14 years and has proven to be a very efficient method for table-top generation of bright THz pulses [59, 66-72]. With it, the generation of $\mathrm{THz}$ pulses with $\mu J$ energies and near-MV/cm peak field strengths has been achieved when pumping by multi-mJ NIR pulses. Possibilities for further 
scaling up to tens-of-mJ and $100 \mathrm{MV} / \mathrm{cm}$ levels have been discussed [67]. To my knowledge, the highest optical rectification generated $\mathrm{THz}$ pulse energy reported so far exceeds $0.4 \mathrm{~mJ}[72]$.

In the TPFP scheme, the intensity front of the NIR pump pulse is tilted by an angle $\gamma$ inside the nonlinear crystal via grating diffraction, and the crystal is cut at this angle as well to allow the induced $\mathrm{THz}$ radiation to exit at normal incidence. According to Huygens's principle, the propagation direction of the generated $\mathrm{THz}$ radiation is perpendicular to the intensity front of the pump (i.e. at an angle $\gamma$ relative to the propagation direction of the pump). If $\gamma$ satisfies [59],

$$
v_{N I R}^{g r} \cos \gamma=v_{T H z}
$$

the $\mathrm{THz}$ wave propagates with a fixed phase relative to the pump intensity front, radiation produced at different times and positions along the pump front adds coherently, and efficient nonlinear conversion is obtained.
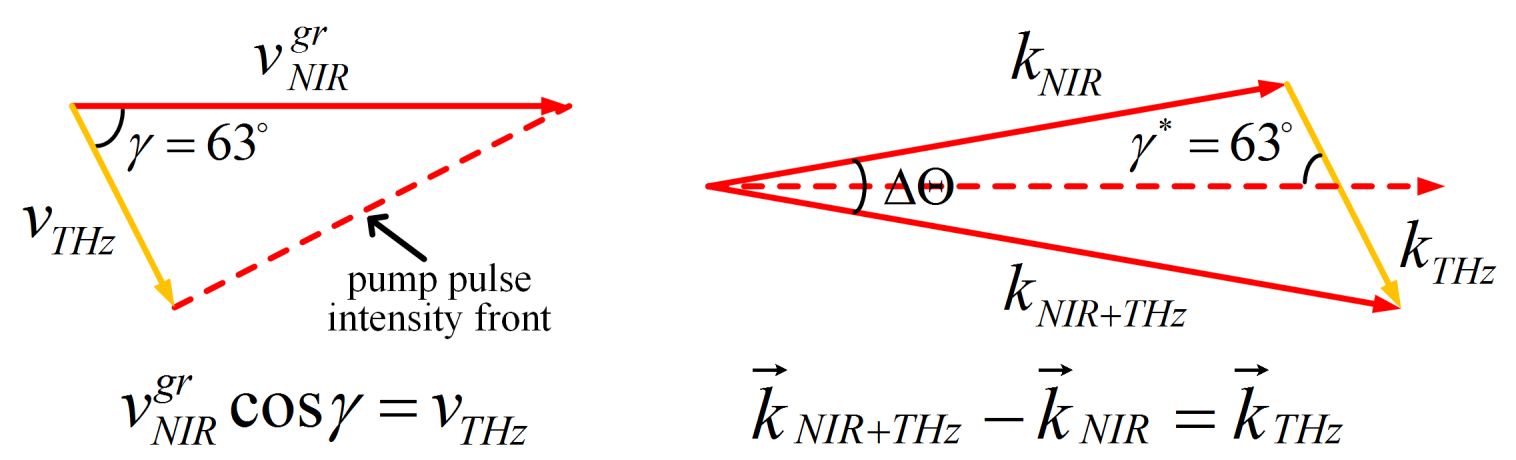

Figure 2.2: Phase matching via tilted-pulse-front-pumping, adapted from Ref. [59].

The TPFP phase matching can also be understood by looking at the DFG process. Diffraction of the broad bandwidth NIR laser pulse from the grating introduces not only an intensity front tilt $\gamma$, but also angular dispersion $\frac{\mathrm{d} \Theta}{\mathrm{d} \omega}$. The relationship between 
$\gamma$ and $\frac{\mathrm{d} \Theta}{\mathrm{d} \omega}$ is given by [73],

$$
\tan \gamma=-\frac{\bar{n}_{N I R}}{n_{N I R}^{g r}} \bar{\omega}_{N I R} \frac{\mathrm{d} \Theta}{\mathrm{d} \omega}
$$

The following non-collinear phase matching conditions must be satisfied for efficient DFG between dispersed frequency components of the NIR pulse,

$$
\left\{\begin{array}{l}
\frac{k_{N I R+T H z}}{\sin \left(\gamma^{*}+\Delta \Theta / 2\right)}=\frac{k_{N I R}}{\sin \left(\gamma^{*}-\Delta \Theta / 2\right)}=\frac{k_{T H z}}{\sin \Delta \Theta} \\
\omega_{N I R+T H z}-\omega_{N I R}=\Omega_{T H z}
\end{array}\right.
$$

Here $\gamma^{*}$ is the angle between propagation direction of the THz pulse and "average" propagation direction of the NIR pulse. Making the small angle approximation in Eq. 2.8a gives,

$$
\begin{gathered}
\left\{\begin{array}{l}
k_{N I R+T H z} \cdot \Delta \Theta=k_{T H z}\left(\sin \gamma^{*}+\Delta \Theta / 2 \cdot \cos \gamma^{*}\right) \\
k_{N I R} \cdot \Delta \Theta=k_{T H z}\left(\sin \gamma^{*}-\Delta \Theta / 2 \cdot \cos \gamma^{*}\right)
\end{array}\right. \\
\Rightarrow\left\{\begin{array}{l}
k_{N I R+T H z}-k_{N I R}=k_{T H z} \cos \gamma^{*} \\
\bar{k}_{N I R} \cdot \Delta \Theta=k_{T H z} \sin \gamma^{*}
\end{array}\right.
\end{gathered}
$$

Dividing Eq. 2.9 by Eq. 2.8b gives,

$$
\left\{\begin{array}{l}
v_{N I R}^{g r} * \cos \gamma^{*}=v_{T H z} \quad \text { or } \quad n_{N I R}^{g r}=n_{T H z} * \cos \gamma^{*} \\
-\bar{n}_{N I R} \bar{\omega}_{N I R} \frac{\mathrm{d} \Theta}{\mathrm{d} \omega}=n_{T H z} \sin \gamma^{*}
\end{array}\right.
$$

Eq. 2.10a is essentially the equation of non-collinear velocity matching. Finally, dividing Eq. 2.10b by 2.10a gives,

$$
\tan \gamma^{*}=-\frac{\bar{n}_{N I R}}{n_{N I R}^{g r}} \bar{\omega}_{N I R} \frac{\mathrm{d} \Theta}{\mathrm{d} \omega}
$$

It is clear, by comparing Eq. 2.7 and Eq. 2.11, that the angle $\gamma^{*}$ between the propagation directions of the THz and NIR pulses is the same as the NIR pulse front tilt angle $\gamma$.

In the experiments, the NIR pump pulse front tilt angle $\gamma$ is $63^{\circ}-65^{\circ}$, while 
the angular spread of the light beam is about $2^{\circ}-5^{\circ}$, therefore the small angle approximation used in the preceding derivation is valid [59].

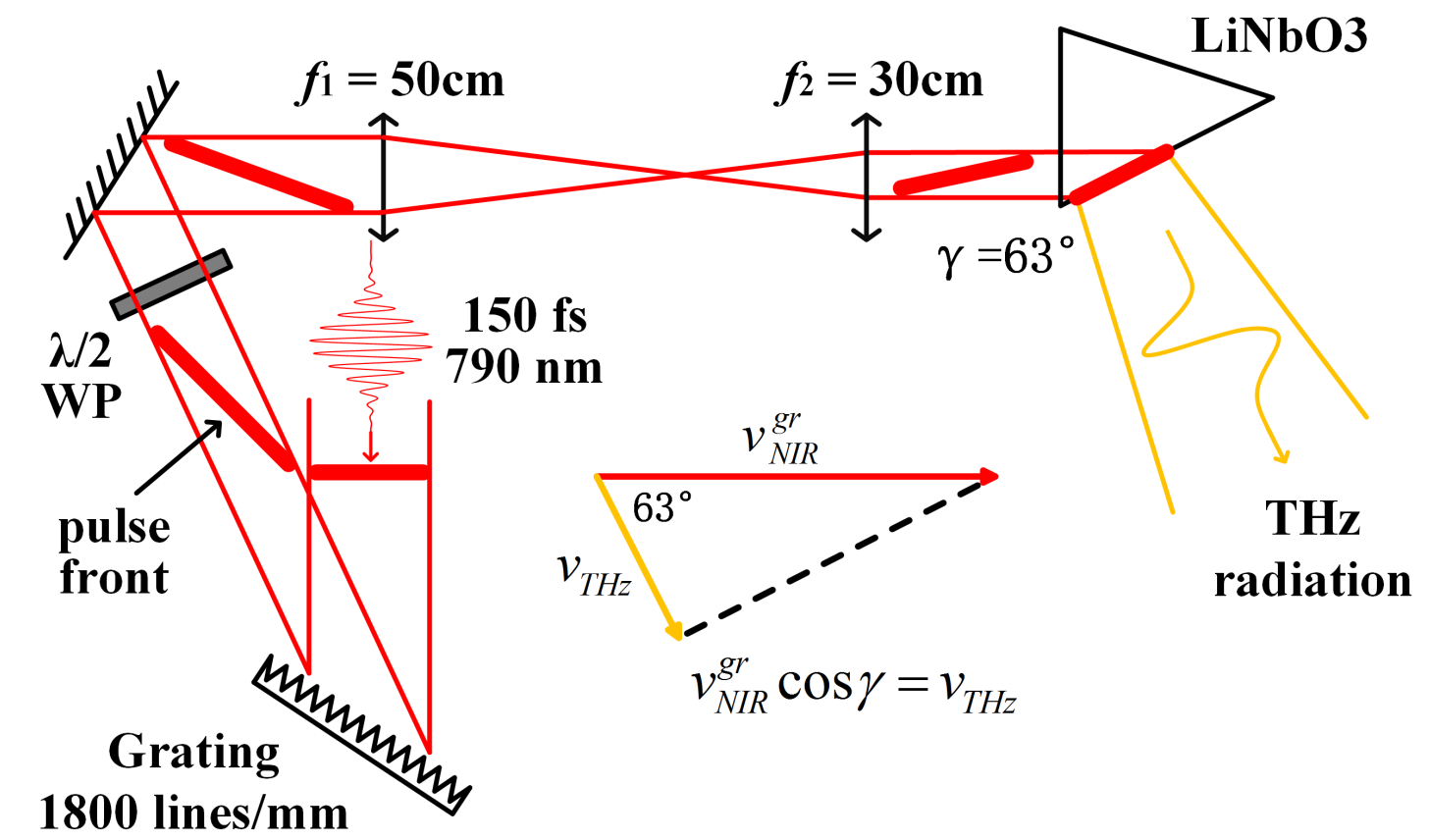

Figure 2.3: Schematic of the experimental setup for $\mathrm{THz}$ generation via tilted-pulse-front-pumping optical rectification. Optimal $\mathrm{THz}$ beam characteristics and pump to $\mathrm{THz}$ conversion efficiency are reached when the tilt angle of the grating image inside the $\mathrm{LiNbO}_{3}$ coincides with that of the pump pulse front [66, 69], from which the grating angle and de-magnification can be calculated. To obtain a tilt angle $\gamma=64^{\circ}$ with a $1800 / \mathrm{mm}$ grating, the calculated optimal de-magnification and grating angle are $\sim 0.59$ and $\sim 35.3^{\circ}$, respectively. In the experiments, a demagnification of 0.6 , and a grating angle of $\sim 33.6^{\circ}$ are used.

\subsubsection{Electro-optic sampling}

Obtaining the THz temporal profile by electro-optic sampling (EOS) is essentially a THz-NIR cross-correlation measurement. The THz pulse modifies the index ellipsoid of the electro-optic crystal, changes the indices of refraction in proportion to its instantaneous field strength, thereby altering the polarization of the NIR pulse. By sampling the polarization change as a function of the delay between the $\mathrm{THz}$ and 
NIR pulses, the temporal shape of the THz pulse can be obtained. EOS is based on the linear electro-optic effect (Pockels effect), which is a second order $\left(\chi^{(2)}\right)$ nonlinear process that results in a linear change in refractive indices as a function of the applied electric field.

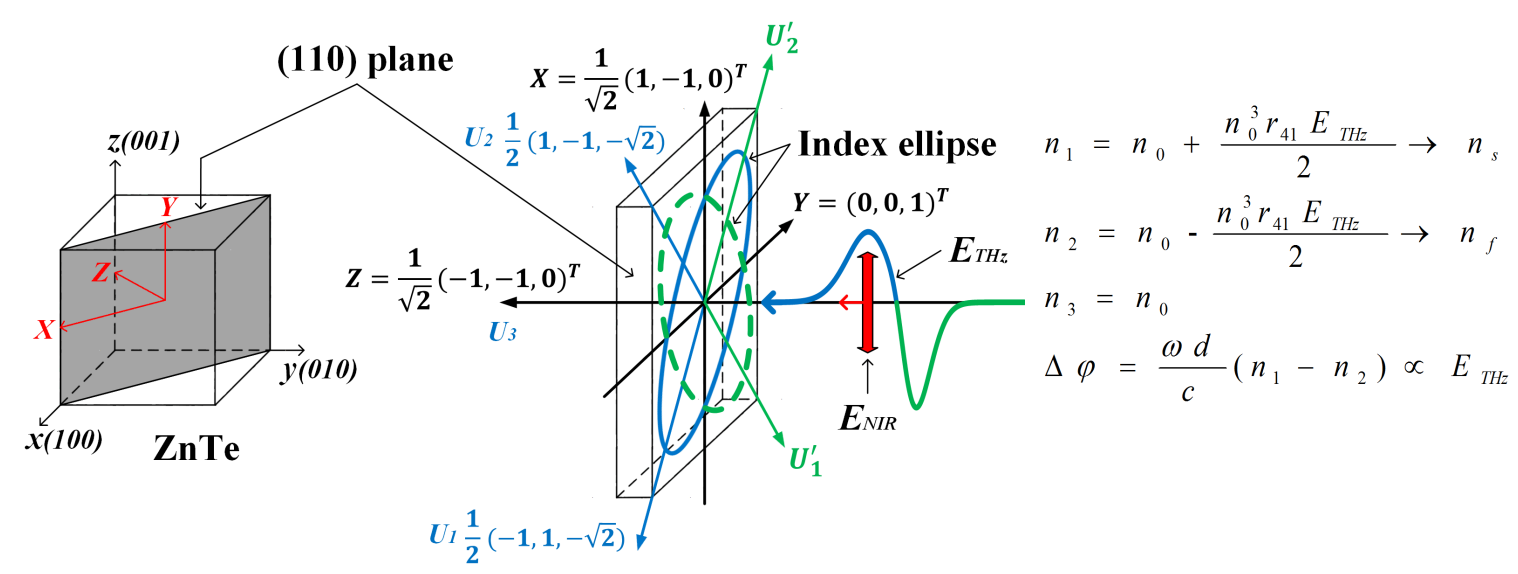

Figure 2.4: Principle of electro-optic sampling. The THz pulse modifies the index ellipsoid of the electro-optic crystal, which alters the polarization of the NIR pulse. (Index ellipses in blue and green colors correspond to cases with the $\mathrm{THz}$ field vector in the $\mathbf{X}$ and $-\mathbf{X}$ directions, respectively. $\mathbf{U}_{i}$ are the modified principle axes.)

Fig. 2.4 illustrates the principle of EOS: A $d=1 \mathrm{~mm}$ thick, (110) cut ZnTe crystal is used as the EOS medium. This crystal is optically isotropic in vanishing electric fields. By applying a $\mathrm{THz}$ pulse polarized along the $\mathbf{X}$ axis, and propagating along the $\mathbf{Z}$ axis, the modified index ellipse has its principal axes at $\pm 45^{\circ}$ relative to $\mathbf{X}$. The field also induces a proportional change in refractive indices $\Delta n_{1,2}= \pm \frac{n_{0}{ }^{3} r_{41} E_{T H z}}{2}$. Here $r_{41}$ is the Pockels coefficient, which for ZnTe is about $4 \mathrm{pm} / \mathrm{V}$ at THz frequencies [74, 75]. Since the NIR pulse is polarized along the $\mathbf{X}$ axis, a phase shift $\Delta \varphi$ is introduced between the two polarization components (along $\mathbf{U}_{1}$ and $\mathbf{U}_{2}$ ) of its electric vector $\mathbf{E}_{N I R}$,

$$
\Delta \varphi=\frac{\omega_{N I R} d}{c}\left(n_{s}-n_{f}\right)=\frac{\omega_{N I R} d}{c} n_{0}^{3} r_{41} E_{T H z} \propto E_{T H z}
$$

The THz field can be determined by "measuring" the phase shift. The detection 
setup consists of: the $\mathrm{ZnTe}$ crystal, a $45^{\circ}$ (relative to $\mathbf{X}$ ) rotated quarter wave plate (QWP), a wollaston prism (WP) (or polarizing beam splitter (PBS)) and a balanced photodiode detector. Fig. 2.5 is a schematic of the setup. Without the THz field, the NIR probe pulse is circularly polarized after the QWP and the balanced diode detector signal is zero. With the THz field, the ZnTe acts as a field dependent $\Delta \varphi\left(E_{T H z}\right)$ phase retarder and the NIR probe pulse becomes elliptically polarized after the QWP. The signal of the balanced diode detector in this case is calculated to be,

$$
\text { Sig. } \propto E_{N I R}^{2} \sin (\Delta \varphi) \propto \sin (\Delta \varphi)
$$

Note it is important to keep the $\mathrm{THz}$ field small so that the phase shift does not exceed $\pi / 2$. (For a $1 \mathrm{kV} / \mathrm{cm}$ field, Eq. 2.12 gives an induced phase shift of $\sim 4^{\circ}$ ). If the phase shift is small, we can apply the small angle approximation to Eq. 2.13 and, recalling Eq. 2.12, the signal is found to be proportional to the THz field,

$$
\text { Sig. } \propto E_{T H z}
$$

A $\pi / 2$ phase shift can be calibrated by adding one more QWP after the ZnTe and rotating it to record the balanced photodiode detector signal in the absence of the $\mathrm{THz}$ field (Maximum positive/negative signal correspond to $\pm \pi / 2$ phase shift, which occurs when the fast axes of the two QWPs are aligned or crossed at an angle of $\pi / 2$ ).

A detailed derivation of the electro-optic sampling can be found in appendix E, as well as in Ref. [74-76].

We have performed THz-NIR electro-optic sampling experiments to measure the waveform of the THz generated via TPFP optical rectification from our setup, and typical results are shown in Fig. 2.6. As shown, the THz pulse has a single-cycle waveform with a period of about $4 \mathrm{ps}$, which is much longer than the duration of the optical pump pulse ( $\sim 150 \mathrm{fs})$. Sources for this broadening (the generation of a 
much longer pulse than that expected from optical rectification process) include the dispersion of both the pump and the $\mathrm{THz}$ pulses inside the crystal and the diffraction of the THz beam after exiting the crystal. Also, the Gouy phase shift when the $\mathrm{THz}$ pulse passes through focus may influence the pulse shape as well.

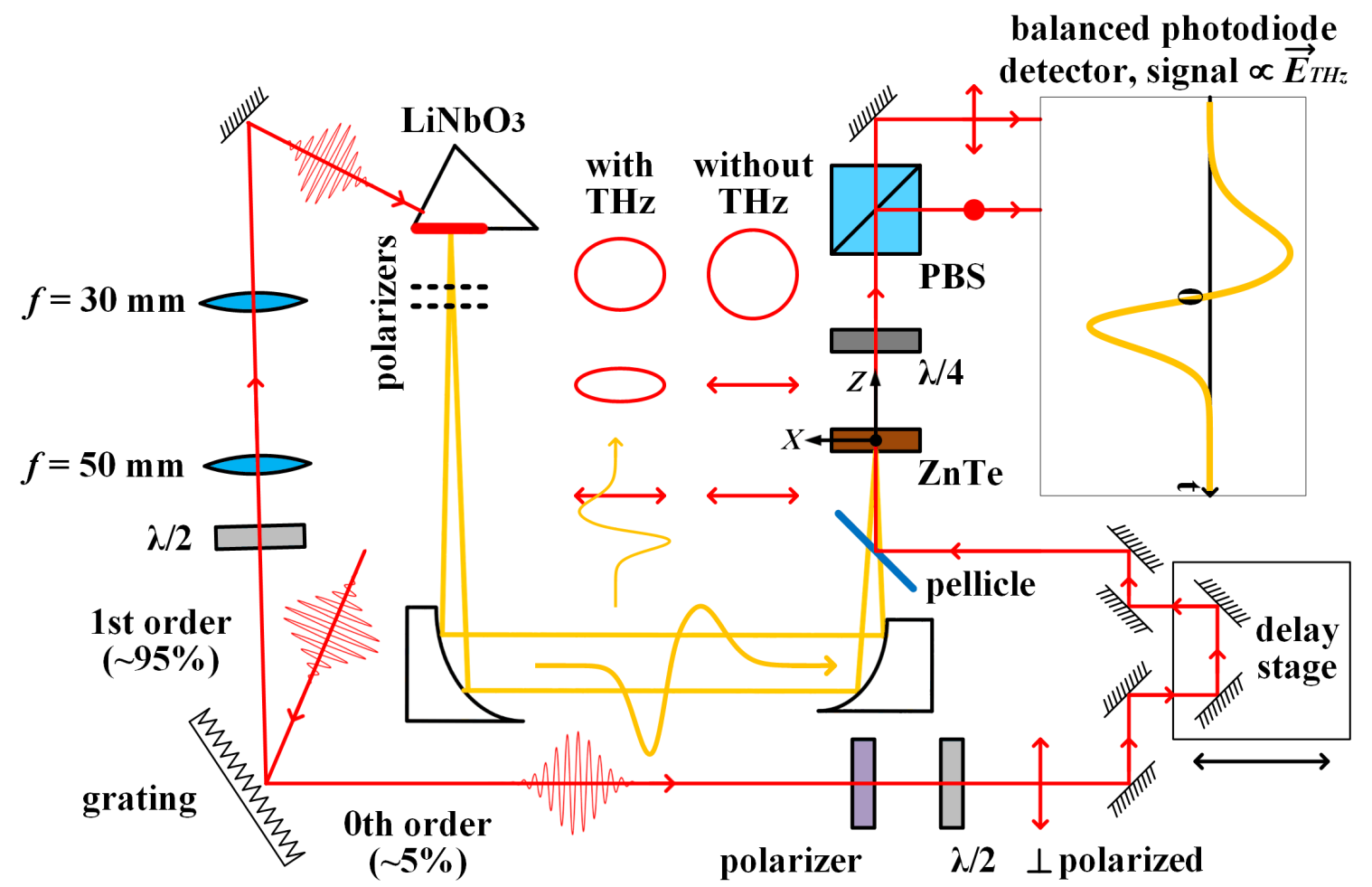

Figure 2.5: EOS experimental setup. $\lambda / 2$ : half wave plate; $\lambda / 4$ : quarter wave plate; PBS: polarizing beam splitter. Note the incident NIR pulse to the grating is horizontally polarized, whereas the incident $\mathrm{THz}$ and NIR pulses to ZnTe crystal are both vertically polarized. The polarization changes are shown horizontally for demonstration purpose. 
a

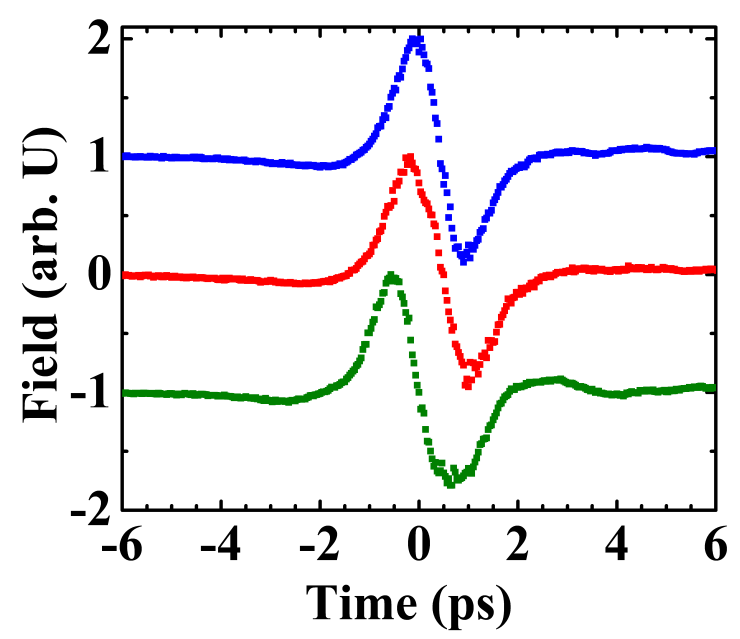

C

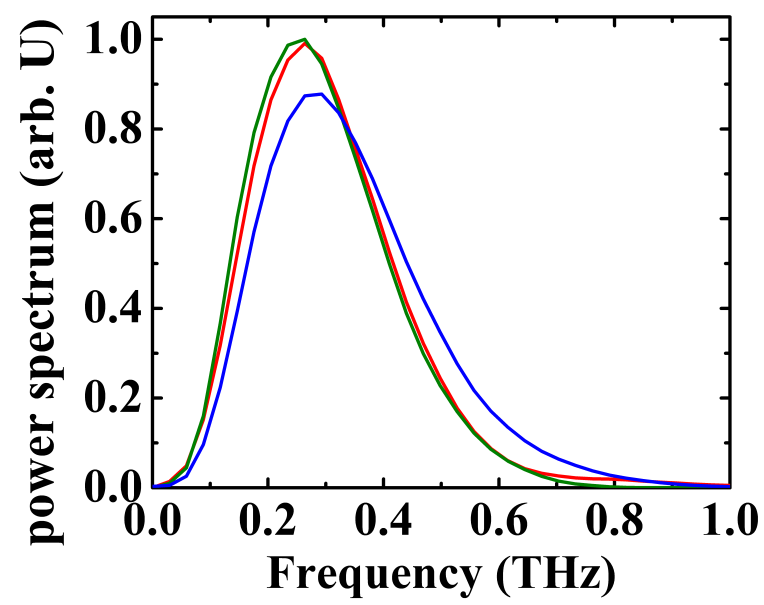

b

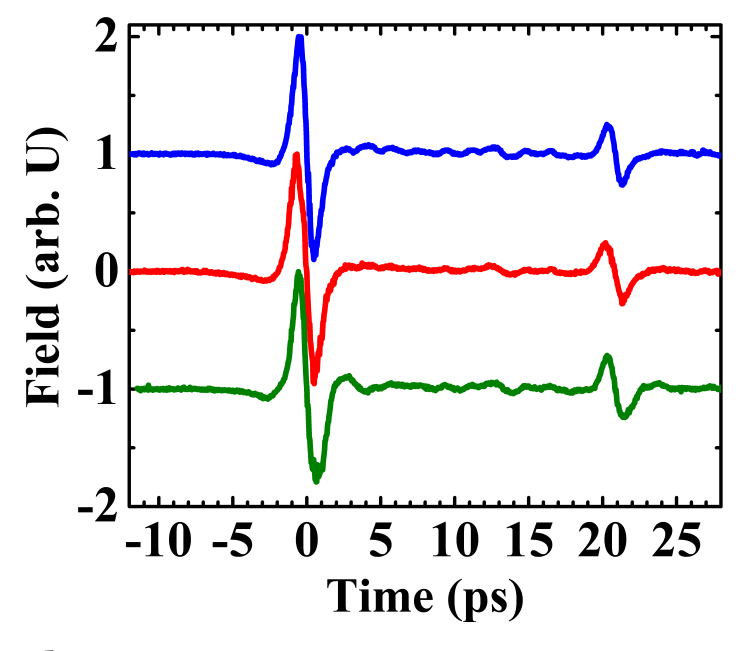

d

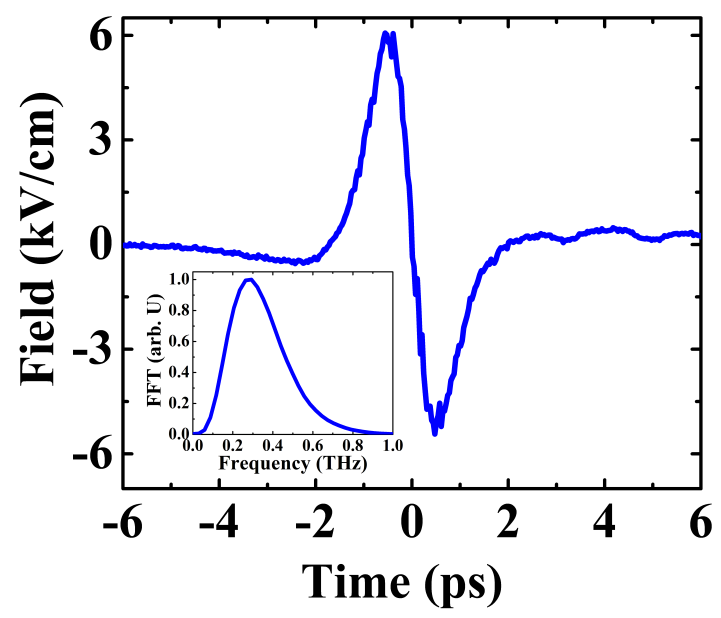

Figure 2.6: Electro-optic sampling experimental results.

a) 3 EOS scans show single cycle $\mathrm{THz}$ waveforms with frequency $\omega_{T H z} \simeq 0.25 \mathrm{THz}$. The field maxima are all scaled to one and the field zeros are shifted for clarity. We have examined the output of $\mathrm{THz}$ generated by $\mathrm{LiNbO}_{3}$ pumped with several NIR pulses: (blue) positive chirp $\sim 250 \mathrm{fs}$, (red) shortest pulse duration $\sim 150 \mathrm{fs}$, (green) negative chirp $\sim 250 \mathrm{fs}$. As shown, the overall shape of the $\mathrm{THz}$ waveform does not change appreciably, but the max field strength obtained with the shortest pump pulse is about 3 times bigger than with chirped pulses.

b) Longer time scans show an extra $\mathrm{THz}$ peak, this is due to $\mathrm{THz}$ reflections inside the ZnTe crystal. Ideally, a thinner ZnTe crystal and lower $\mathrm{THz}$ field are preferred for EOS measurements to reduce unwanted nonlinearities.

c) FFT power spectrum of data in a).

d) $\mathrm{THz}$ field calculated from the measured phase shift, same as the blue trace in a). 


\subsubsection{Streaking spectroscopy}

A conventional streak camera maps the temporal (or temporal and spatial) intensity profile of an optical pulse onto one (or two) spatial axes of a "slow" imaging detector [77]. The "streaking" can be achieved either mechanically, by using a rotating mirror or moving slit system to deflect the light, or electronically, by deflecting photoelectrons produced by the light pulse using a time-dependent field. Mechanical streaking provides high spatial resolution but is limited in temporal resolution, whereas (conventional) electronic streaking has superb temporal resolution (state-ofthe-art commercial designs allows a temporal resolution of around $200 \mathrm{fs}$ [78]) but with limited spatial resolution.

Pump-probe all optical streaking offers a way of directly accessing the electric field of a light wave. A pump pulse photo-ionizes a sample, and the photoelectrons interact with a probe or "streak" pulse. The electric field of the streak pulse can be deduced by sampling the momentum transfer (from the streak field to the photoelectrons) as a function of time delay between the pump and streak pulses. Moreover, information about the temporal dynamics of the photoionization/photoemission process are encoded in the streaked photoelectron spectra. Indeed, decoding this information is at the core of streaking spectroscopy which has played a key role in the development of attosecond science (See the review articles [79-81] and references therein). To clarify, in one configuration, electrons produced by an ionizing pump pulse "probe" the electric field of the streak pulse. In a different scenario, the streak field "probes" the photoemission/photoionization dynamics of the sample.

For a pump pulse with pulse duration much shorter than the period of the streak pulse, i.e. $\tau_{\text {pump }} \ll T_{\text {streak }}$, the magnitude of the streak field is essentially "frozen" within the time interval $\tau_{p u m p}$, and the photoelectrons can be considered as instantly 
launched into the streak field [82]. Assuming the photoelectrons are free of any spatially dependent electric potential, The momentum transfer from the streak field to the photoelectrons is,

$$
\Delta p(\boldsymbol{r}, t)=-\int_{t}^{\infty} F_{\text {streak }}\left(\boldsymbol{r}, t^{\prime}\right) \mathrm{d} t^{\prime}=\alpha(A(\boldsymbol{r}, \infty)-A(\boldsymbol{r}, t))
$$

here $F_{\text {streak }}(\boldsymbol{r}, t)$ and $A(\boldsymbol{r}, t)$ are the electric field and vector potential of the streak pulse, respectively, and $\alpha$ is the fine structure constant. $\Delta p$ is zero if the pump and streak pulses are very far apart, accordingly, without loss of generality, we can define $A(\boldsymbol{r},-\infty)=A(\boldsymbol{r}, \infty)=0$ such that,

$$
\Delta p(\boldsymbol{r}, t)=-\alpha A(\boldsymbol{r}, t)
$$

Eq. 2.16 is the essence of simpleman's model and, in principle, can be trivially inverted to give the electric field of the streak pulse,

$$
F(\boldsymbol{r}, t)=\frac{\mathrm{d}}{\mathrm{d} t}(\Delta p(\boldsymbol{r}, t))
$$

The first demonstration of ultrafast light field measurement with the (NIR(fs)XUV(as)) optical streaking technique was carried out in 2004 [82]. A $\lambda=750 \mathrm{~nm}$, $\tau_{F W H M}=4.3$ fs ultrafast few-cycle laser pulse was characterized using a 250 attosecond XUV pulse. See figure 2.7 and figure 2.8 for details.

In this dissertation, we have measured the $\mathrm{THz}$ waveform using $\mathrm{THz}(\mathrm{ps})$-fs optical streaking [83]. The measurements also support an examination of strong-field energy transfer in the single-cycle limit. A detailed description is included in Chap. 3. 


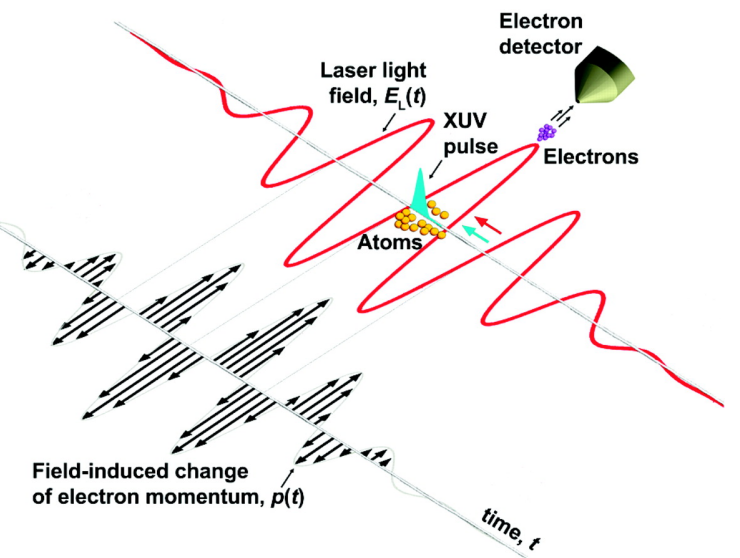

Figure 2.7: NIR-XUV optical streaking light field measurement: Principle. (from Ref. [82]). A few-cycle pulse of laser light, together with a synchronized subfemtosecond XUV burst, is focused into an atomic gas target. The XUV pulse knocks electrons free by photoionization. The light field $E_{L}(t)$ to be measured imparts a momentum change to the electrons (black arrows), which scales as the instantaneous value of the vector potential $A_{L}(t)$ at the instant of release of the probing electrons. The momentum change is measured by an electron detector, which collects the electrons ejected along the direction of the linearly polarized $E_{L}(\boldsymbol{r}, t)$.
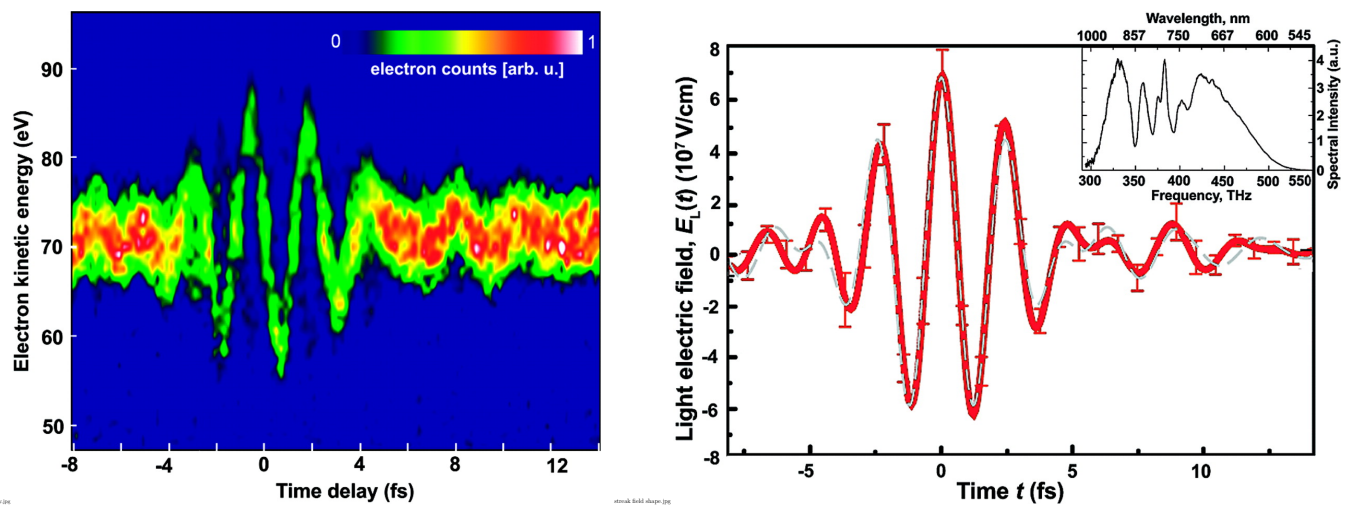

Figure 2.8: NIR-XUV optical streaking light field measurement: Streaked electron energies and derived NIR light field. (from Ref. [82]). Left) A series of kinetic energy spectra of electrons detached by a 250-as, 93-eV XUV pulse from neon atoms in the presence of an intense $<5$-fs, 750-nm laser field, in false-color representation. The delay of the XUV probe was varied in steps of 200 as, and each spectrum was accumulated over $100 \mathrm{~s}$. The detected electrons were ejected along the laser electric field vector with a mean initial kinetic energy of $p_{i}^{2} \approx \hbar \omega_{X U V}-W_{b}=$ $93 \mathrm{eV}-21.5 \mathrm{eV}=71.5 \mathrm{eV}$. The energy shift of the electrons versus the timing of the XUV trigger pulse that launches the probing electrons directly represents $A_{L}(t)$. Right) $E_{L}(t)$ reconstructed (red line) from the data depicted in the left figure and calculated (gray line) from the measured pulse spectrum (inset) with the assumed absence of a frequency-dependent phase and with $E_{0}$ and $\varphi$ chosen so as to afford optimum matching to the measured field evolution. 


\subsection{Dye Lasers}

Dye lasers use fluorescing organic dyes, usually in the form of liquid solution, as the gain medium. Due to their broad wavelength tunablility (typically $30-100 \mathrm{~nm}$ ), and wide range of emission spectra, from near-ultraviolet to near-infrared (311-1800 nm) with over 500 different dyes [84], dye lasers are widely applied in scientific research. There are several categories of dye lasers, such as CW dye lasers, ultrashort pulsed dye lasers, and narrow linewidth dye lasers, etc. [85]. In this dissertation, narrow linewidth pulsed dye lasers have been used.

a
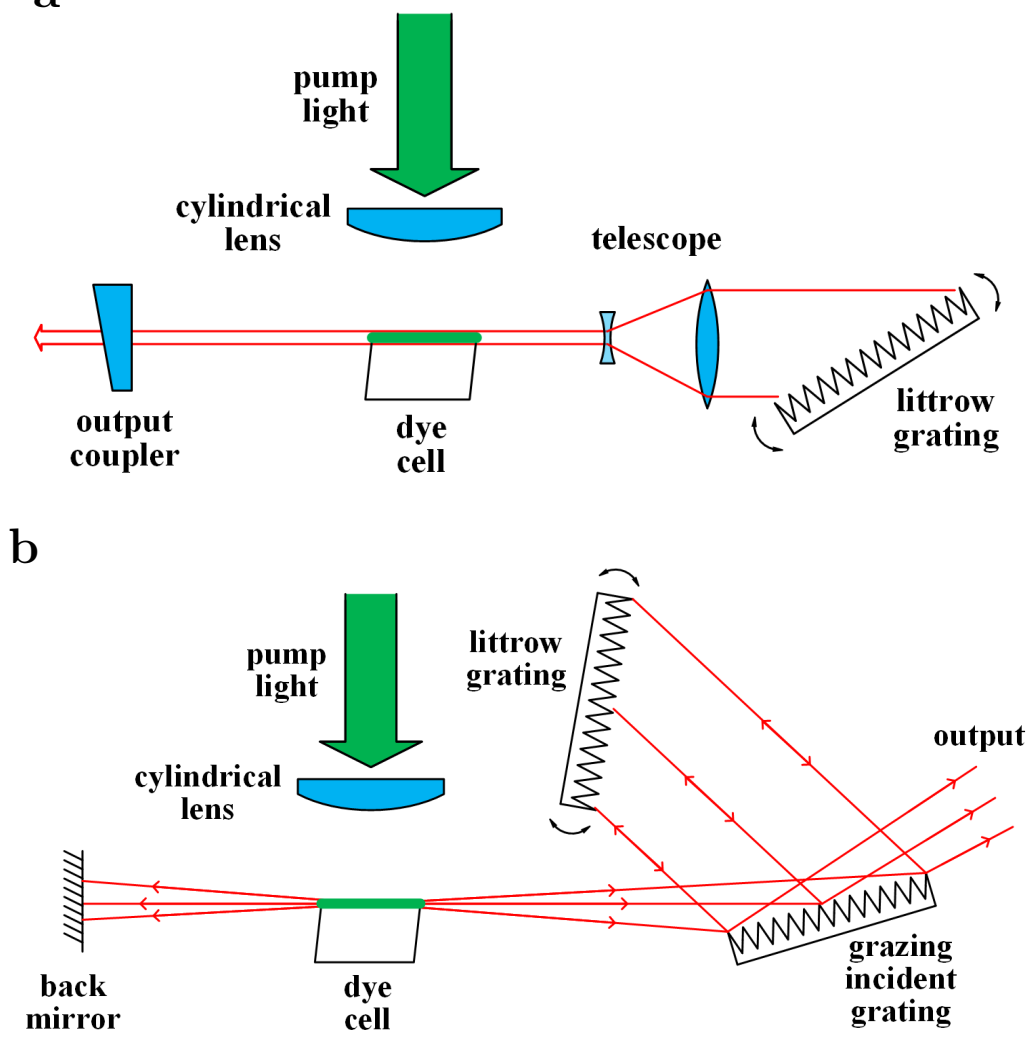

\section{Figure 2.9: Schematics of a) Hänsch and b) Littman dye lasers.}

There are two basic designs of narrow linewidth pulsed dye lasers, which are named after their designers, Hänsch [86] and Littman [87]. Typical schematics are shown in Figs. 2.9a and 2.9b. Narrow linewidth is achieved by expanding the beam (with 
telescope or grazing grating incidence) and selecting the desired wavelength (using the angular dispersion of a diffraction grating) to oscillate in the cavity. Advanced designs, such as prismatic beam expansion and intra-cavity etalons, can be used to get even narrower linewidth. State-of-the-art commercial pulsed dye lasers reach linewidths below $0.03 \mathrm{~cm}^{-1}(900 \mathrm{MHz})$ at $570 \mathrm{~nm}$, without any intra-cavity etalons $[88,89]$.

Both Hänsch and Littman dye lasers, self-constructed, have been used in this dissertation to drive Rydberg excitations for the THz-induced Na ionization experiments described in Chap. 3 and Chap. 4. The optical energy source, or pump, is provided by the $2 \mathrm{nd}(532 \mathrm{~nm})$ or $3 \mathrm{rd}(355 \mathrm{~nm})$ harmonic of $\sim 10 \mathrm{~ns}$ pulsed Nd:YAG laser. Typical linewidths for the lasers used are about $1 \mathrm{~cm}^{-1}$ and $0.25 \mathrm{~cm}^{-1}$ for the Hänsch and Littman designs, respectively.

\subsection{Photoelectron spectroscopy}

Measuring the energy of electrons typically involves analyzing the effects of electrostatic forces $\mathbf{F}=-e \mathbf{E}$ and/or magnetic forces $\mathbf{F}=-e \mathbf{v} \times \mathbf{B}$ on them, and/or mapping motional characteristics onto other measurable quantities (time, displacement, etc.). Two such instruments have been used in this dissertation: a time-of-flight electron spectrometer and a retarding potential energy analyzer.

\subsubsection{Time-of-flight electron spectrometer}

A time-of-flight (TOF) electron spectrometer determines the kinetic energy $E_{k}$ of electrons by mapping momentum into time $t$. After traveling a distance (namely the drift path $L$ ) in a field free region, electrons with different momenta arrive at a detector at different times. An oscilloscope records the electron yield $\Delta N$ as a 
function of the arrival time, and the energy spectrum can be obtained by performing a variable transformation to the probability density function $\left(P_{p d f}\right)$,

$$
\begin{gathered}
E_{k}(t)=\frac{m_{e} L^{2}}{2 t^{2}} \quad \text { or } \quad t\left(E_{k}\right)=\sqrt{\frac{m_{e} L^{2}}{2 E_{k}}} \\
P_{p d f}\left(E_{k}\right)=P_{p d f}(t)\left|\frac{\mathrm{d} t}{\mathrm{~d} E_{k}}\right|=P_{p d f}(t) \sqrt{\frac{m_{e} L^{2}}{8}} E_{k}^{-3 / 2} \\
\frac{\Delta N(t)}{N_{0}}=\int_{t}^{t+\Delta t} P_{p d f}\left(t^{\prime}\right) \mathrm{d} t^{\prime}=P_{c d f}(t+\Delta t)-P_{c d f}(t) \\
P_{p d f}(t)=\frac{\mathrm{d} P_{c d f}(t)}{\mathrm{d} t}=\lim _{\Delta t \rightarrow 0} \frac{P_{c d f}(t+\Delta t)-P_{c d f}(t)}{\Delta t}=\frac{1}{N_{0}} \lim _{\Delta t \rightarrow 0} \frac{\Delta N(t)}{\Delta t}
\end{gathered}
$$

Here $m_{e}$ is the electron mass, $P_{c d f}(t)=\int_{-\infty}^{t} P_{p d f}\left(t^{\prime}\right) \mathrm{d} t^{\prime}$ is the cumulative distribution function, and $N_{0}=\sum_{t} \Delta N(t)$ is the normalizing factor. In fact, we can directly perform the variable transformation on the recorded data $\Delta N(t)$ as it is essentially the histogram representation of $P_{p d f}(t)$,

$$
\Delta N\left(E_{k}\right)=\Delta N(t)\left|\frac{\mathrm{d} t}{\mathrm{~d} E_{k}}\right|=\Delta N(t) \sqrt{\frac{m_{e} L^{2}}{8}} E_{k}^{-3 / 2}
$$

From equation 2.18a the energy resolution of the spectrometer is,

$$
\frac{\delta_{E_{k}}}{E_{k}}=2 \sqrt{\left(\frac{\delta_{t}}{t}\right)^{2}+\left(\frac{\delta_{L}}{L}\right)^{2}}
$$

Typically a TOF electron spectrometer needs to be calibrated prior to usage, mainly to determine the time zero $t_{0}$ (the "birth" time of photoelectrons relative to the trigger time of the scope), and some geometric parameters of the spectrometer.

A self-built TOF electron spectrometer has been used in the experiments described in Chap. 3, to measure the energy of electrons from THz-fs streaking and from $\mathrm{THz}$ induced field ionization of Rydberg atoms. The energy resolution of the spectrometer is mainly limited by the temporal resolution of the data collection oscilloscope (LeCroy $9350 \mathrm{~A}$, bandwidth $500 \mathrm{MHz}$, maximum sample rate $1 \mathrm{GS} / \mathrm{s}$ ). As measured with this 
oscilloscope, the electron signal has a $10 \%-90 \%$ rise time of $\sim 1.5$ ns. Thus, form Eq. 2.20 , the resolution is $\Delta E_{k} \simeq 5 \mathrm{eV}$ at $E_{k} \simeq 100 \mathrm{eV}$ and $\Delta E_{k} \simeq 6 \mathrm{meV}$ at $E_{k} \simeq 1$ $\mathrm{eV}$, respectively.

A schematic of the TOF spectrometer, as well as its calibration, can be found in Appendix F.

\subsubsection{Retarding potential energy analyzer}

A retarding potential energy analyzer (RPA) determines the kinetic energy $E_{k}$ of electrons by applying a retarding voltage $V_{r}$ and filtering out electrons with energies below the cutoff potential $E_{k}<e V_{r}=U_{r}$. Thus, the analyzer serves as a high-pass electron energy filter. An oscilloscope records the electron yield $N$ as a function of the cutoff potential and the energy spectra can be obtained by differentiation,

$$
\begin{gathered}
\left.N\left(E_{k}\right)\right|_{U_{r}}=\int_{U_{r}}^{\infty} P_{p d f}\left(E_{k}\right) d E_{k}=1-\int_{-\infty}^{U_{r}} P_{p d f}\left(E_{k}\right) d E_{k}=1-\left.P_{c d f}\left(E_{k}\right)\right|_{U_{r}} \\
\left.P_{p d f}\left(E_{k}\right)\right|_{U_{r}}=\left.\frac{\mathrm{d} P_{c d f}\left(E_{k}\right)}{\mathrm{d} E_{k}}\right|_{U_{r}}=-\left.\frac{\mathrm{d} N\left(E_{k}\right)}{\mathrm{d} E_{k}}\right|_{U_{r}} \\
=-\left.\lim _{\Delta E_{k} \rightarrow 0} \frac{N\left(E_{k}+\Delta E_{k}\right)-N\left(E_{k}\right)}{\Delta E_{k}}\right|_{U_{r}}
\end{gathered}
$$

In the experiments described in Chap. 5, a self-built RPA has been used to measure the energy of electrons from THz-induced field emission. The resolution of the RPA is on the order of several eV. A schematic and more detail about the RPA can be found in Appendix F. 


\subsection{Sample preparation}

\subsubsection{Sodium Oven}

For the experiments described in Chaps. 3 and 4, the thermal atomic beam of $\mathrm{Na}$ is produced by heating a $\mathrm{Na}$ filled oven, which is essentially a stainless steel tube half filled with a few grams of solid $\mathrm{Na}$, and crimped on both ends with a small side hole drilled near the tube's center for beam output. Upon heating, $\mathrm{Na}$ atoms are vaporized and effuse from the side hole. The solid $\mathrm{Na}$ is usually stored in mineral oil. Before filling the oven tube, several Na pieces with a fairly thin oxide layer are cut into smaller pieces to expose the unoxidized portion. These pieces are then rinsed with toluene to remove excess mineral oil. The oven tube is made of 316 steel, length/thickness/OD is $6 " / 0.007^{\prime \prime} / 0.25^{\prime \prime}$, side hole diameter is $0.04 \mathrm{~mm}$. The total resistance of the tube is approximately $0.1 \Omega$. The heating current supply consists of a set of $6.3 \mathrm{~V} \mathrm{AC}$ filament transformers, wired in parallel, with a Variac autotransformer that controls the voltage/current supplied to the oven. For Na, a typical heating current is $10-25$ Amps. To shield the chamber from thermal radiation, and to confine the effusive atoms to a beam, the oven is enclosed in a water-cooled copper shield. Atoms exit through a $\sim 4 \mathrm{~mm}$ diameter hole in the shield (see Fig. 2.15). The shield aperture and the oven side hole define the axis of the atomic beam. The typical atomic density 10 $\mathrm{cm}$ away from the oven is estimated to be $10^{7}-10^{8}$ atoms $/ \mathrm{cm}^{3}$.

\subsubsection{Tungsten nano-tips}

The tungsten nano-tips used in the experiments described in Chap. 5 are prepared via electrochemical etching: Tungsten wire (99.95\% purity, $0.25 \mathrm{~mm}$ diameter), a graphite rod (99.995\% purity, $0.25^{\prime \prime}$ diameter) and $3 \mathrm{~mol} / \mathrm{L} \mathrm{NaOH}$ solution $(\mathrm{NaOH}$ 
dissolved in distilled water) serve as the anode, cathode and electrolyte, respectively. The $\mathrm{W}$ anode immersion depth is $2 \mathrm{~mm}$ and the etching voltage is 4 Volts. The radius of the etched tip is controllable by varying the "post-etching" time, i.e., how long the etching voltage remains after the primary etching has completed.

As shown in Fig. 2.10, when the $\mathrm{W}$ wire is immersed into the electrolyte, a meniscus is formed around it at the air-electrolyte interface due to surface tension. The etching rate is the fastest around the meniscus region. The etching process starts when a positive voltage is applied to the wire, and "primary etching" ends when the immersed portion of the wire drops off because the tensile strength cannot sustain the weight of the submerged portion of the wire.

Typically, the etching starts with a current of $20-25 \mathrm{~mA}$, primary etching ends at a current of $6-9 \mathrm{~mA}$ after $5-7$ mins. There is a sudden current drop to $<1$ $\mathrm{mA}$ when wire "drop-off" occurs. By monitoring either the wire "drop-off" or the sudden current drop by eye, and manually switching off the etching voltage in 0 (immediately), 1-2, 3-5, or 6-10 seconds, tips with radii of 100-200, 200-300, 400-600, or 700-1000 nm, respectively, are produced. A fast switch (circuit diagram can be found in Appendix G) that automatically switches off the voltage within several $\mu$ s upon wire "drop-off" has been built to produce ultra-sharp tips with 10-50 nm tip radius.

To avoid oxidation, as soon as possible after etching, the tungsten tips are imaged using a scanning electron microscope (SEM). Tips with the desired radius and cone angle are selected and mounted in the vacuum chamber for experiments.

Here are a few "tips" on tungsten nano-tip preparation: a) $2 \mathrm{~mol} / \mathrm{L} \mathrm{NaOH}$ works as fine as $3 \mathrm{~mol} / \mathrm{L} \mathrm{NaOH}$ but with longer etching time (about 10-11 mins) to prepare one tip. b) Everything (beakers, tweezers, graphite rod etc.) needs to be cleaned with distilled water prior to usage. c) Tungsten wires to be etched need to be polished, and 
rinsed with first isopropanol, then distilled water in the ultrasonicator. Etched wires need to be taken out from the $\mathrm{NaOH}$ solution as soon as possible and rinsed with first distilled water, then isopropanol in the ultrasonicator for about $1 \mathrm{~min}$, then stored in isopropanol. d) Do NOT clean ultra-sharp tips $(r<30 \mathrm{~nm})$ using the ultrasonicator because they can be easily bent in the ultrasonic vibrations. e) Vibration is unwanted during etching, because it may break the meniscus and produce tips with multi-step shapes. f) It was found that sometimes the etching rate is very unstable, which produces "ugly" shaped tips. This is probably due to insufficient mixing of $\mathrm{NaOH}$ solution. For better results, prepare the solution one day ahead, let the beaker that contains the solution vibrate in ultrasonicator for 1 hour, while occasionally stirring with a glass rod. g) To obtain high magnification SEM images, the wires need to be very steadily mounted on the sample holder to avoid vibration. An easy way is to "clamp" the wire between two layers of carbon tape (see Fig. 2.11Right). Isopropanol is needed to remove the sticky carbon tape from the wire.

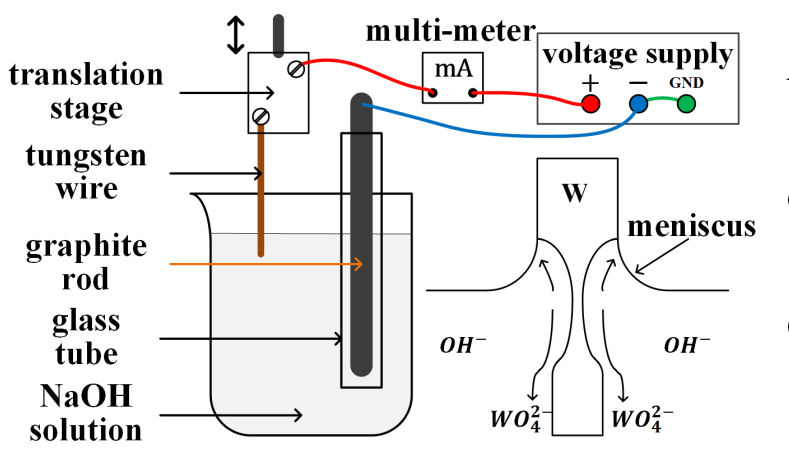

Anode:

$$
\mathrm{W}(\mathrm{s})+8 \mathrm{OH}^{-} \rightarrow \mathrm{WO}_{4}^{2-}+4 \mathrm{H}_{2} \mathrm{O}+6 \mathrm{e}^{-}
$$

Cathode:

$$
6 \mathrm{H}_{2} \mathrm{O}+6 e^{-} \rightarrow 3 \mathrm{H}_{2}(g) \uparrow+6 O \mathrm{H}^{-}
$$

Overall:

$W(s)+2 \mathrm{OH}^{-}+2 \mathrm{H}_{2} \mathrm{O} \rightarrow 3 \mathrm{H}_{2}(g) \uparrow+W O_{4}^{2-}$

Figure 2.10: Electrochemical etching setup and operation principle. 

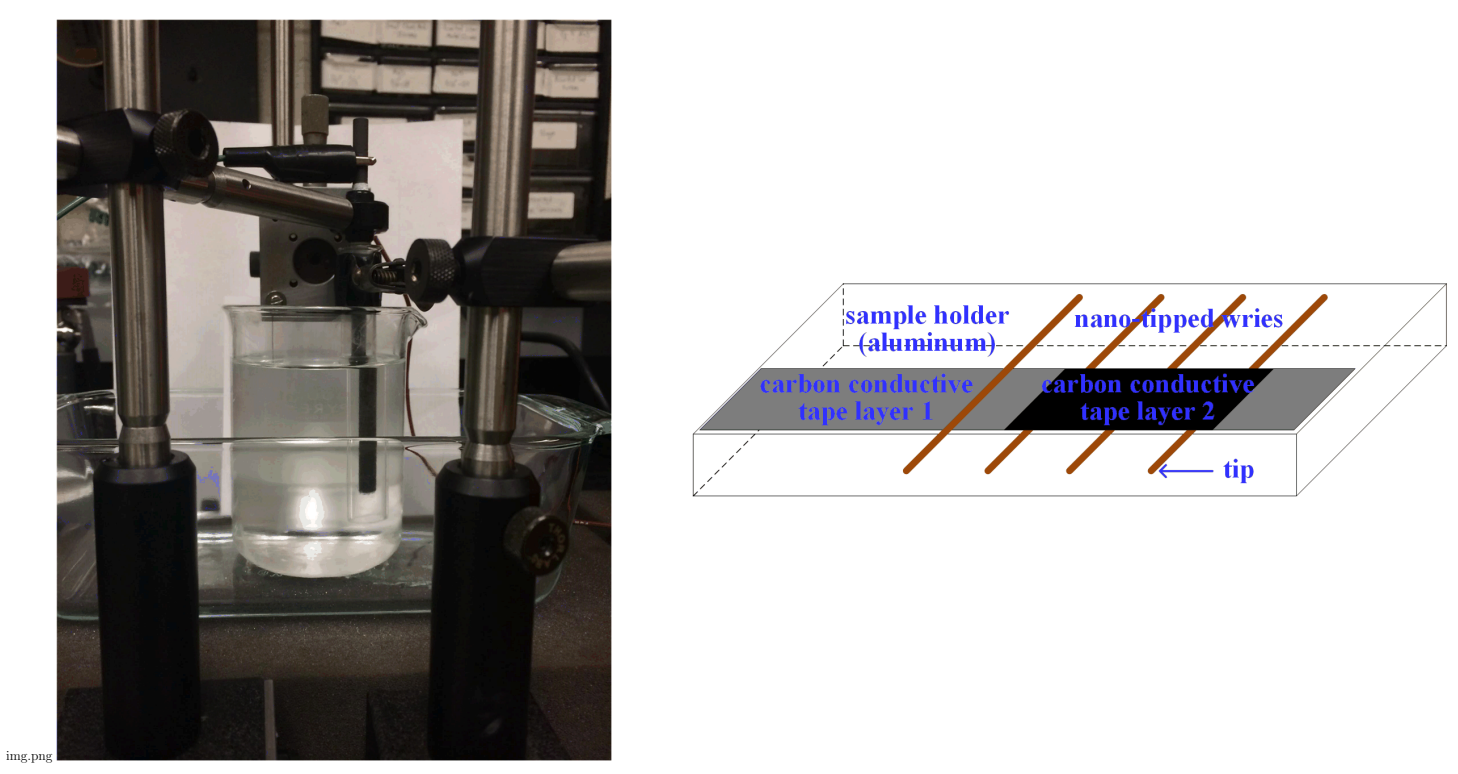

Figure 2.11: Left) Electrochemical etching photo view. Right) Demonstration of mounting the wires on the SEM sample holder.

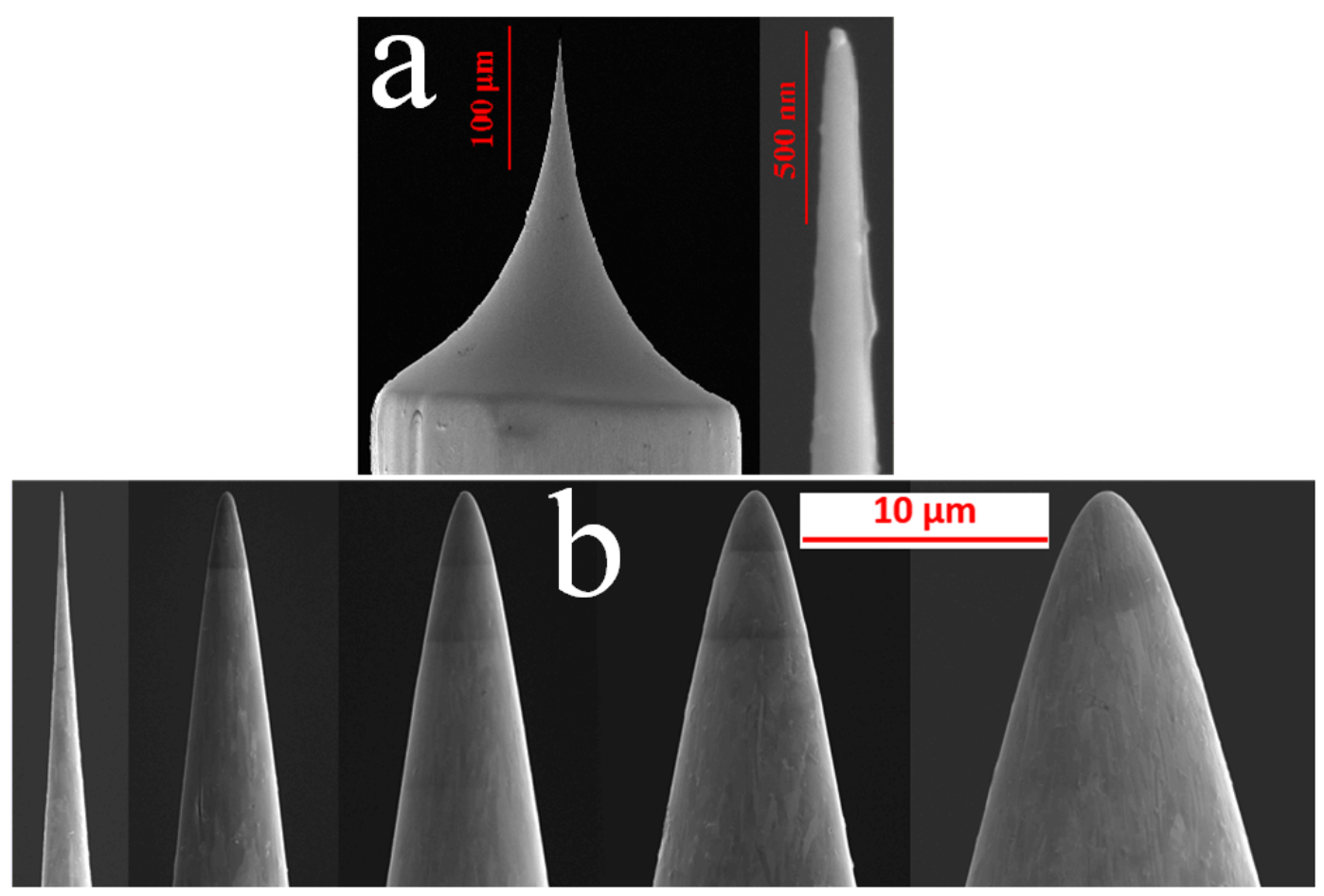

Figure 2.12: SEM images of electrochemically etched tungsten nano-tips. a) $300 \times$ image showing typical overall shape of a electrochemically etched tip and $80 \mathrm{k} \times$ image showing the close look of a sharp tip. b) $5 \mathrm{k} \times$ images of 5 tips with various radii, $\mathrm{r} \simeq(20,100,200,400,800) \mathrm{nm}$, respectively. 


\subsection{Vacuum System}

The laser-matter interaction experiments described in this dissertation are performed in a vacuum chamber. Fig. 2.13 is a schematic of the vacuum system exterior. Fig. 2.14 provides a a view of the inside of the vacuum chamber. Fig. 2.15 shows the laser-matter interaction region.

The main part of the cylindrical shaped aluminum chamber is $\sim 45 \mathrm{~cm}$ in diameter and $\sim 30 \mathrm{~cm}$ in height, with 8 side flanges and 5 top flanges (on the lid) for attachments or extensions. All flanges are sealed with Buna-N or Viton O-rings. The chamber is pumped by a Varian VHS-6 diffusion pump with its foreline backed by a Welch 1376 rotary mechanical pump. The chamber base pressure can be as low as $\sim(2-3) \times 10^{-7}$ Torr. When liquid nitrogen cold traps are used, the pressure can be lowered to $\sim 1 \times 10^{-7}$ Torr. The Rydberg atomic field ionization experiments (Chap. 3 and Chap. 4) did not require ultra-high vacuum and were performed at a base pressure of $\sim 2 \times 10^{-6}$ Torr, due to a leak that was not discovered until after the experiments were completed. The tungsten field emission experiments (Chap. 5) were performed at a base pressure of $\sim 1 \times 10^{-7}$ Torr. (Ideally a non-oil pumped ultra-high vacuum chamber would be preferred for field emission experiments, mainly to avoid sample contamination.) 


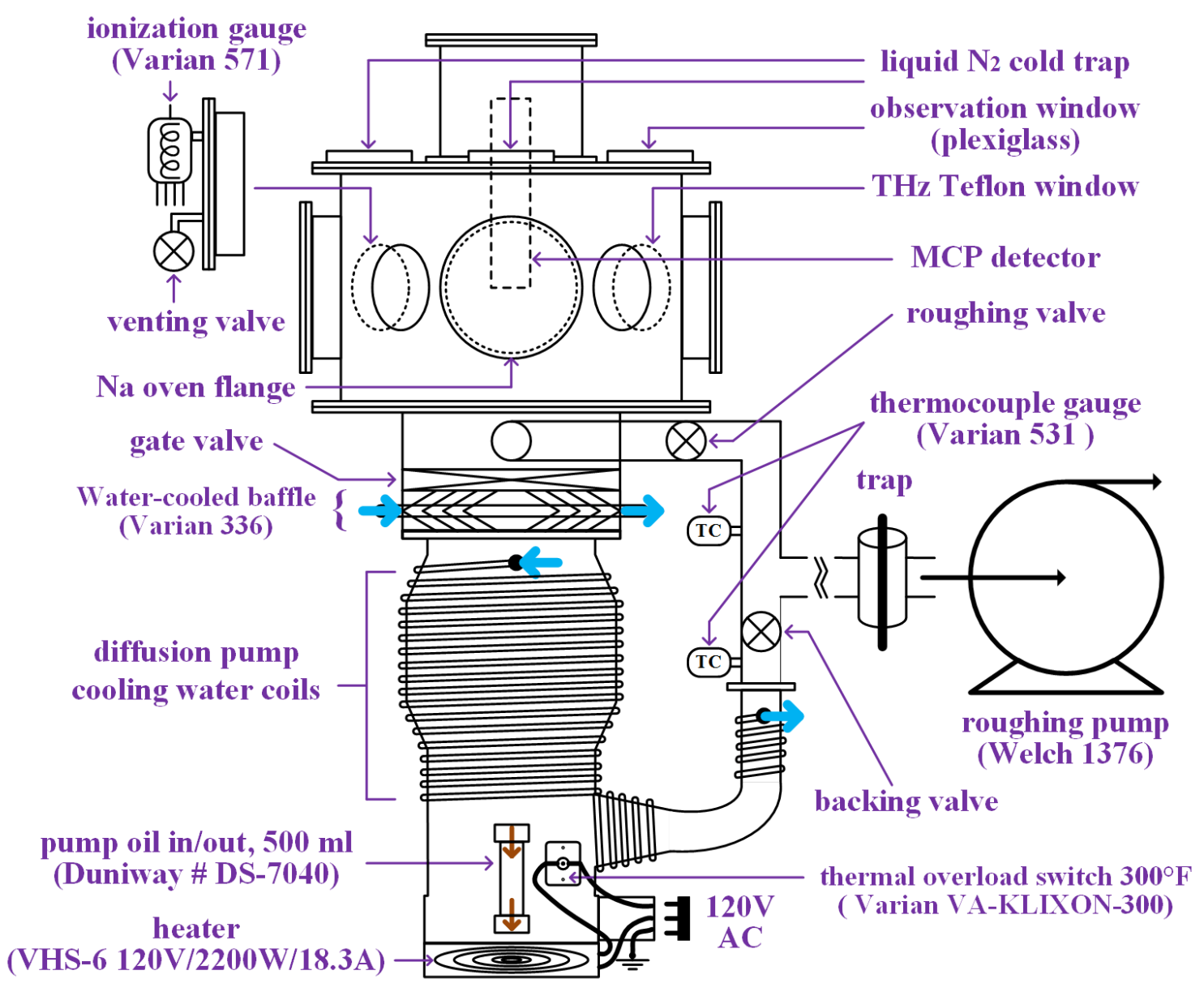

Figure 2.13: Schematic of the vacuum system exterior. A water-cooled baffle between the chamber and the diffusion pump lowers the heat radiation and intercepts primary backstreaming. A oil vapor trap between the roughing pump and the foreline prevents back migration of pump oil vapor. A Varian diffusion pump thermal overload switch avoids overheating. A self-built interlock circuit turns off the diffusion pump heater and closes the backing valve upon power outage. Pressures are measured with ionization gauge and thermocouple gauges, and monitored by a Varian 843 gauge controller. 

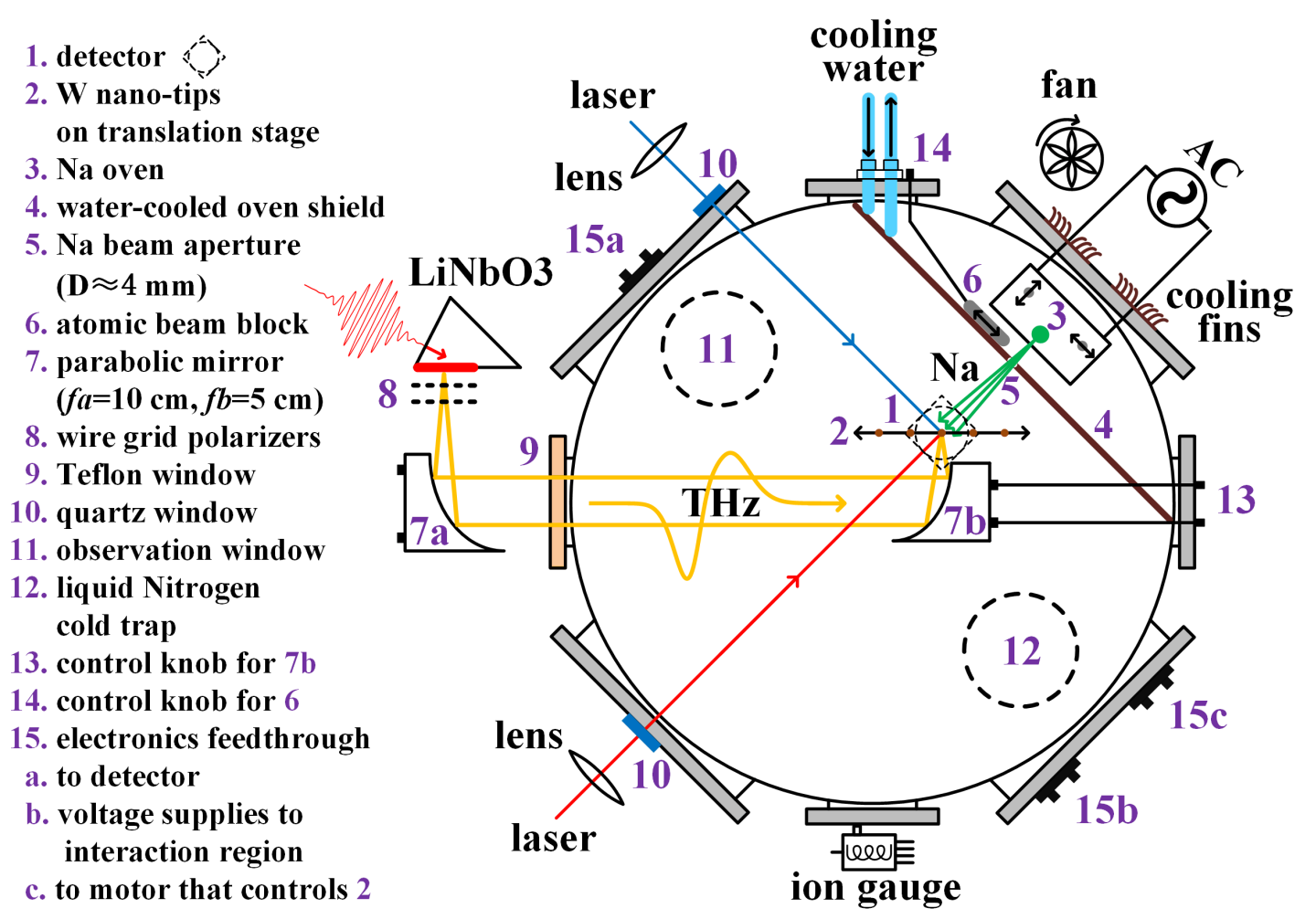

Figure 2.14: Schematic of setups inside the vacuum chamber, top view. Note that the $\mathrm{Na}$ atomic beam and $\mathrm{W}$ nano-tips are for different experiments and do not co-exist. The laser beams serve the following functions: 1) The crossing point of the lasers helps to mark the position of the $\mathrm{THz}$ focus, as $\mathrm{THz}$ radiation is invisible. The focal size of the lasers is $\sim 100 \mu \mathrm{m}$, whereas that of the $\mathrm{THz}$ is about $2 \mathrm{~mm}$, hence the laser focus also defines the interaction region. 2) In the Na atomic fieldionization experiments, the (dye) lasers are used to drive Rydberg transitions. 3) In the $\mathrm{W}$ nano-tip field emission experiments, the lasers are only used for visualizing the alignment of the tips but not for the actual experiments. The alignment can be monitored from the observation window (11).
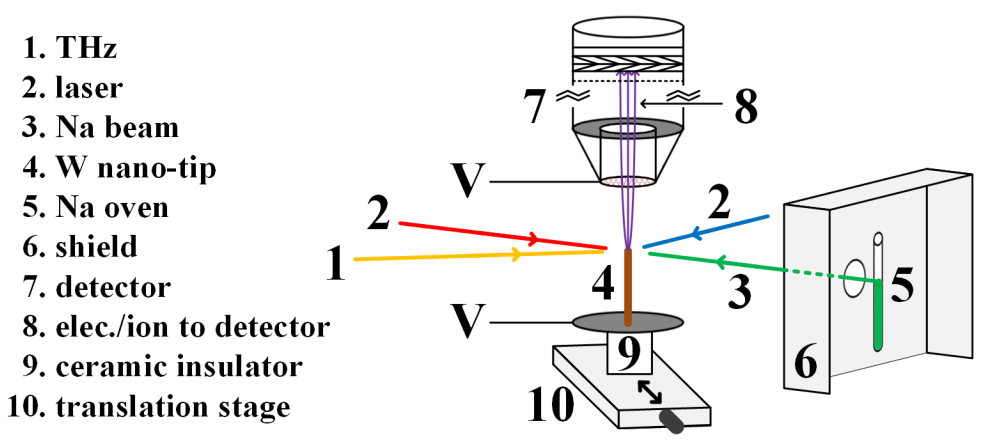

Figure 2.15: Schematic of the interaction region, side view. 


\title{
Chapter 3
}

\section{Ionization of Excited Atoms by}

\section{Intense Single-Cycle THz Pulses}

\begin{abstract}
We have employed intense, single-cycle $\mathrm{THz}$ pulses to explore strong-field ionization of low-lying Na Rydberg states in the low-frequency limit. At the largest fields used, $F \simeq 430 \mathrm{kV} / \mathrm{cm}$, electrons with energies up to $60 \mathrm{eV}$ are created. The field ionization threshold is greater than expected for adiabatic "over-the-barrier" ionization and is found to scale as $n^{-3}$. In addition, for a given field amplitude, higher energy electrons are produced during the ionization of the most tightly bound states. These observations can be attributed to the suppression of scattering from the non-hydrogenic ion core, the long times required for Rydberg electrons to escape over the barrier in the field-dressed Coulomb potential, and the failure, in the single-cycle limit, of the standard prediction for electron energy transfer in an oscillating field. The latter, in particular, holds important implications for future strong-field experiments involving the interaction of ground-state atoms and molecules with true single-cycle laser fields.
\end{abstract}


The exaggerated properties of Rydberg atoms, including small binding energies, high state densities, and long time scales, make them excellent systems for exploring atomic ionization dynamics in pulsed and oscillating fields [10]. A variety of fieldionization mechanisms have been identified in the rf [90-96], microwave [90, 97, 98], and $\mathrm{THz}$ [99] spectral regions. Different mechanisms dominate the ionization process in different regimes, delineated by comparing the time and frequency scales in the field to the corresponding natural atomic scales. Measurements of requisite ionization fields vs $n$ identify boundaries between ionization regimes, and play a key role in the discovery of new ionization pathways. Several of these are noted here and reviewed in more detail below. For example, in the long-pulse/low-frequency regime, overthe-barrier (OTB) ionization involving little or no energy transfer from the field to the electron occurs with an $n^{-4}$ threshold field scaling [10, 91-93]. Conversely, in a short unipolar pulse, ionization occurs by impulsive energy transfer to the electron, requiring a larger field proportional to $n^{-1}[95,96,100]$. More complex dynamics are reflected in other $n$-dependencies, e.g., $n^{-5}$ [97] or $n^{-2}$ [99].

Beyond fundamental interest, insights gleaned from Rydberg atom measurements play an important role in understanding strong-field processes involving groundstate atoms or molecules, from tunneling or over-the-barrier ionization [101], to nonadiabatic ladder climbing [102, 103], to the development [22-24] and refinement [104] of the simpleman's model of energy transfer to electrons in an oscillating field.

Prior to describing our results on ionization with intense single-cycle pulses, we first review the ionization mechanisms noted above. For convenience we use the concept of scaled frequency, defined as $\Omega=\omega / \omega_{k}=\omega n^{3}$ where $\omega$ is the laser frequency and $\omega_{k}=n^{-3}$ is the atomic Kepler orbital frequency, to classify different ionization regimes. 
$\Omega \ll 1$ : The quasi-static regime and Over-the-barrier ionization. [10]

In the long-pulse/low-frequency regime, one important ionization pathway is classical over-the-barrier ionization (OTBI), which occurs if the field

$$
F \geq \frac{E^{2}}{4 Z}
$$

such that the electron energy lies above the saddle point of the combined CoulombStark potential. Here $E$ is the electron energy and $Z$ is the net charge of the ionic core. For hydrogen, $E_{n}=-1 /\left(2 n^{2}\right)$ and $Z=1$, and the ionization threshold field is,

$$
F_{\text {thresh. }}=\frac{\left(-1 / 2 n^{2}\right)^{2}}{4}=\frac{1}{16 n^{4}}
$$

Note Eq. 3.1 and Eq. 3.2 are only valid for $m=0$ states. The threshold fields of $m \neq 0$ states are higher than that of the $m=0$ states due to the centrifugal potential, which keeps the electrons away from the field axis where the saddle point of the potential is located.

Eq. 3.2 ignores the field induced Stark energy shift, as well as the spatial distribution of the electronic wavefunction. We now consider how both of these affect the OTBI threshold for different Stark states. As discussed in Chap. 1, in parabolic coordinates, the electron motion in the $\xi$ direction is bounded, so for ionization to occur, the electron must escape to infinity along the $\eta$ coordinate (see Fig. 1.3). Accordingly, ionization is classically allowed for energies above the saddle point of the effective potential $V(\eta)$. According to Eq. 1.15, the ionization condition for a low $|m|$ state of hydrogen is approximated to be,

$$
F \geq \frac{[E(F)]^{2}}{4 Z_{2}}
$$

where $Z_{2}$ is the effective charge binding the electron in the $\eta$ coordinate. The extreme red Stark state of high n, $m=0$ of hydrogen has $Z_{2} \approx 1$, and its energy is adequately 
given considering the linear Stark shift, $E_{n}(F) \simeq-1 /\left(2 n^{2}\right)-3 n^{2} F / 2$. Therefore the ionization threshold field for the extreme red Stark state of $\mathrm{H}$ is calculated to be,

$$
F_{\text {thresh. }}=\frac{1}{9 n^{4}}
$$

States other than the extreme red Stark state require higher field for ionization, because they have smaller values of $Z_{2}$. As $Z_{2}$ decreases, the energy of the Stark state increases, but so does the saddle point of $V(\eta)$ (see Fig. 1.3), and the electronic wavefunction becomes more and more localized along the bounded coordinate $\xi$ (see Fig. 1.5). Typically, the extreme blue and red Stark states of the same $n$ and $m=0$ have ionization threshold fields differ by a factor of 2 (Fig. 3.1a) [10].
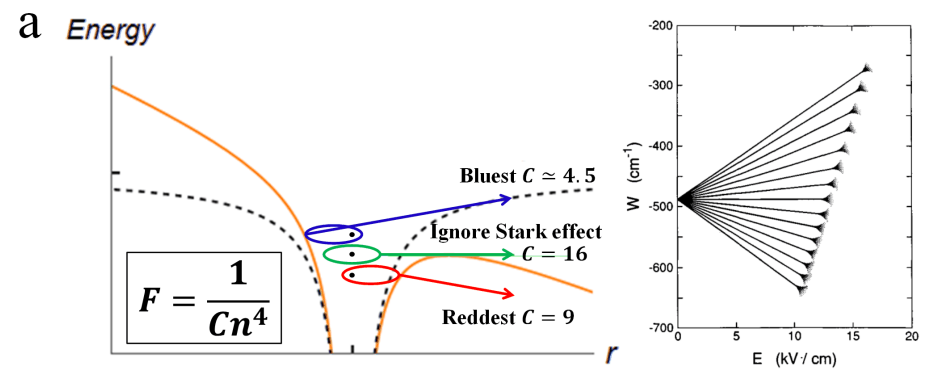

$\mathrm{b}$
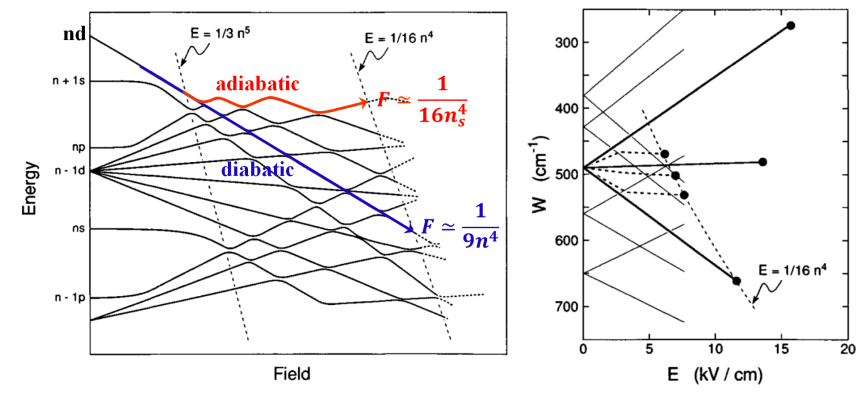

Figure 3.1: Classical over-the-barrier ionization for a) hydrogen and b) non-hydrogenic atoms, adapted from Ref. [10]. Fig. a Left) Illustration of the classical over-the-barrier ionization. Fig. a Right) Energy levels of the $\mathrm{H} \mathrm{n}=15$, $\mathrm{m}=0$ Stark states. The broadening of the levels represents the non-zero ionization rate. Blue states are harder to be ionized than red states. Fig. b Left) Schematic energy level diagram of $\mathrm{Na} m=0$ Stark states and adiabatic and diabatic ionization paths for an initial d state. Fig. b Right) Adiabatic (dashed bold lines) and diabatic (solid bold lines) ionization paths for three $n=15$ states in the center and on the edges of the Stark manifold. Adiabatic ionization always occurs at lower fields than diabatic ionization. 
The OTBI of a non-hydrogenic Rydberg atom, for example an alkali atom, differs from that of $\mathrm{H}$ because of the finite sized ionic core, or equivalently, the non-zero quantum defects. In a non-H Rydberg atom, Stark states exhibit avoided energy crossings. As the system passes from low to high field, depending on how quickly the field rises, state probability amplitude approaching an avoided energy crossing can traverse it adiabatically, diabatically or a mixture of both. For a very slowly rising pulse, all the avoided-crossings are traversed adiabatically, and the atom remains in the same adiabatic energy level prior to ionization. When the field reaches $F=[E(F)]^{2} / 4$, ionization occurs by coupling to the red Stark continua of higher $n$-manifold. This ionization path is known as the adiabatic ionization path (Fig. 3.1b). In contrast, for a relatively fast rising field all avoided-crossings are traversed diabatically, ionization follows the hydrogen-like path (Fig. 3.1b).

Alternatively, the non-hydrogen-like adiabatic or the hydrogen-like diabatic ionization path can be understood in the "core-scattering" point of view. A bound electron can scatter from one Stark-trajectory to another each time it passes near the non-H ionic core. Which path the probability amplitude takes, i.e., the adiabaticity of the avoided-crossing traverse, depends on the core-scattering rate relative to the slew rate of the field.

Considering adiabatic ionization of Stark states in a n-manifold. As the system passes from low to high field, the energy ordering of the zero-field states is preserved, and the energies are approximately trapped within the range $\frac{1}{2(n-1 / 2)^{2}} \leq E(F) \leq$ $\frac{1}{2(n+1 / 2)^{2}}$ (see Fig. 3.1b). Conveniently, we can define an effective quantum number $n_{s}$ such that the energies in the field can be represented by $E(F)=-1 /\left(2 n_{s}^{2}\right)$ as if there is no Stark shift, and the ionization threshold field can be expressed as [10],

$$
F_{\text {thresh. }}=\frac{1}{16 n_{s}^{4}}
$$


If we assume that the energies in the high field are evenly spaced, we may estimate the $n_{s}$ values for states of the $n, m=0$ Stark manifold of $\mathrm{Na}[10,105]$,

$$
\begin{aligned}
(n+1) s & \rightarrow n_{s}=n-1 / 2 \\
n l & \rightarrow n_{s}=n-1 / 2+(l-1) / n \\
(n+1) p & \rightarrow n_{s}=n+1 / 2-1 / n
\end{aligned}
$$

Principles of adiabatic OTBI enable state-selective field ionization (SSFI): For a very slowly ramped ionizing field, each Stark state follows its own adiabatic ionization path, and different states ionize at different field strengths, hence different times of the ramping field. Accordingly, the time-dependent ionization signal reveals the initial state population distribution.

$\Omega \ll 1$ : Many-cycle microwave fields and Landau-Zener ladder climbing. $[10,97]$

For a many-cycle microwave field with $\Omega \ll 1$, low $m$ hydrogen atoms ionize at a field $F=1 /\left(9 n^{4}\right)$, whereas low $m$ alkali atoms can be ionized at a field substantially less than the classical field required for ionization,

$$
F_{\text {thresh. }} \simeq \frac{1}{3 n^{5}}
$$

A simplified description of this microwave ionization process is as follows: The microwave field induces a sequence of Landau-Zener transitions (see Appendix $\mathrm{H}$ for more detail about Landau-Zener transition) between the extreme blue and red Stark levels of adjacent $n$-manifolds, driving the initial population to higher-lying states which can be directly field ionized by the microwave field (Fig. 3.2). The ionization threshold field for this process is the field at which the extreme blue and red Stark states of the $n$ and $(n+1)$ manifolds exhibit an avoided crossing, i.e., $F \simeq 1 /\left(3 n^{5}\right)$. Note that the $n \rightarrow n+1$ transition probability is maximum when the microwave 
frequency $\omega$ is comparable to $\omega_{0}$, the size of the avoided crossing at $F \simeq 1 /\left(3 n^{5}\right)$. If $\omega \gg \omega_{0}$ or $\omega \ll \omega_{0}$, the $n \rightarrow n+1$ transition probability is negligible and ladder climbing will not occur at this threshold.

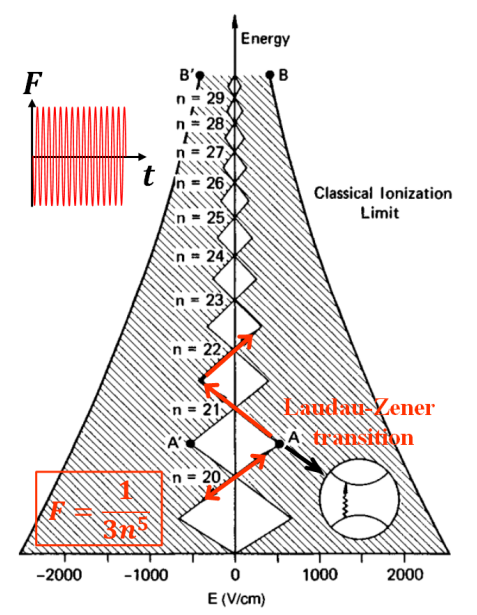

Figure 3.2: An example of microwave field ionization of non-hydrogenic Rydberg atoms, adapted from Ref. [97].

$\Omega \gg 1$ : Half-cycle pulses and Impulsive ionization. $[95,99,100]$

For a half-cycle pulse (HCP) with $\Omega \gg 1$, i.e., in the short-pulse/high-frequency regime, a Rydberg electron experiences a momentum kick from the field. The momentum and energy transfer from the field to the electron are,

$$
\begin{gathered}
\Delta \mathbf{p}=-\int \mathbf{F}(t) \mathrm{d} t \propto F \\
\Delta E=\mathbf{p}_{\mathbf{0}} \Delta \mathbf{p}+(\Delta p)^{2} / 2
\end{gathered}
$$

where $\mathbf{F}(t)$ is the electric field of the HCP and $\mathbf{p}_{\mathbf{0}}$ is the initial momentum of the electron. In the short-pulse regime, the electron has insufficient time to pass over the saddle point during the brief suppression of the binding potential. As a result, ionization can only occur if the energy transferred to the bound electron is greater than its binding energy, $\Delta E \geq 1 /\left(2 n^{2}\right)$. Apparently the amount of the energy transfer depends on the electron's momentum $\mathbf{p}_{\mathbf{0}}$ at the instant of the kick. Maximum energy 
transfer occurs when the electron comes near the ionic core and its velocity reaches the maximum value, and the field direction is anti-parallel to the direction of the electron motion (see Fig. 3.3). In this case the first term of Eq. 3.9 dominates the energy transfer, $\Delta E \simeq \mathbf{p}_{\mathbf{0}} \Delta \mathbf{p}$, and the ionization threshold field scales as,

$$
F_{\text {thresh. }} \propto \frac{1}{n^{2}}
$$

In the sudden limit (an ultrashort-pulse), the motion of the electron is negligible during the field "kick" and we can set $\mathbf{p}_{\mathbf{0}} \simeq 0$. Accordingly, the electron's position is and the atomic potential has no influence on the momentum and energy transfer. In this case the impulse approximation applies, and the energy transfer is determined by second term of Eq. 3.9, $\Delta E \simeq(\Delta p)^{2} / 2$, the ionization threshold field scales as,

$$
F_{\text {thresh. }} \propto \frac{1}{n}
$$

Ionization induced by a HCP in the long-pulse regime $(\Omega \ll 1)$ is the same as the classical OTBI described before, with ionization threshold field scales as,

$$
F_{\text {thresh. }} \propto \frac{1}{n^{4}}
$$
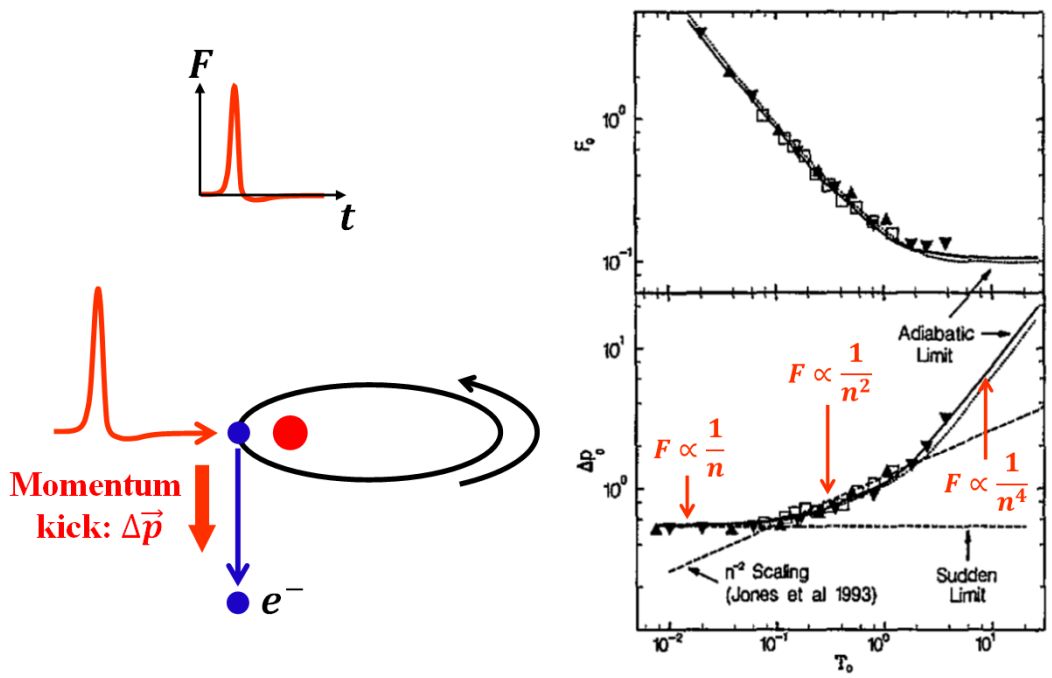

Figure 3.3: Ionization by a half-cycle pulse, adapted from Ref. [95]. 
So as noted at the beginning of this chapter, the $n$-scaling of the ionization threshold field reveals the ionization mechanism. Therefore, measurements of ionization threshold vs. $n$ have played an important role in understanding strong field ionization in different regimes. We now consider such measurements in a previously unexplored regime.

The experiments described in this chapter explore single-cycle THz ionization of low-lying Rydberg states of Na with principle quantum number $6 \leq n \leq 15$. The field period (and duration), $T \simeq 4 \mathrm{ps,} \mathrm{is} \mathrm{longer} \mathrm{than} \mathrm{the} \mathrm{Kepler} \mathrm{period,} \tau_{K}=2 \pi n^{3}$, and the field frequency, $\omega \simeq 0.20 \mathrm{THz}$, is less than the spacing between adjacent $n$-states, $\Delta E_{n} \simeq 1 / n^{3}$, for all levels studied (see Fig. 3.4b for the THz waveform). Thus, based on these atomic scales, the experiments fall in the low-frequency, long-pulse regime $^{1}$. Surprisingly, we find that the threshold fields are greater than expected for adiabatic ionization, and scale as $n^{-3}$ rather than $n^{-4}$. Classical simulations reproduce the observed scaling, attributing it to both the suppression of non-hydrogenic corescattering and the long times, $>\tau_{K}$, that electrons require to travel over the saddlepoint in the field-dressed Coulomb potential.

The electron energy distributions also suggest new physics associated with the single-cycle nature of the ionizing field. We find, counter-intuitively, that electrons originating from the most tightly-bound Rydberg states leave the atom with the greatest energies. Moreover, in spite of the fact that field-driven, high-energy electron backscattering $[19,107,108]$ does not occur in the single-cycle field, the electron energies can significantly exceed the simpleman's prediction, $\Delta E_{\max }=2 U_{p}$ for the maximum allowable energy transfer in the absence of rescattering. Here $U_{p}=F_{\max }^{2} / 4 \omega^{2}$ is the

\footnotetext{
${ }^{1}$ The Keldysh parameter $\gamma=\sqrt{I P} / 2 U_{p}$ is used to delineate the adiabatic and non-adiabatic strong-field ionization boundary for ground-state atoms and molecules. It takes on values $0.1 \leq \gamma \leq$ 0.66 in these experiments, suggesting adiabatic ionization at the fields required to ionize the $n$ states studied. However, $\gamma$ derives from a zero-range potential model and alone is not useful for classifying ionization from extended orbitals [106].
} 
ponderomotive energy of a free-electron in an oscillating field with peak amplitude $F_{\max }$ and frequency $\omega$. These observations reflect the failure of two standard tenets of strong field energy transfer which, in turn, will have a substantial impact on experiments that require distinguishability of direct and rescattered electrons in intense single-cycle laser fields.

In the experiment, $\mathrm{Na}$ atoms in a thermal beam in vacuum are laser excited to nd Rydberg states at the center of a time-of-flight (TOF) spectrometer using two, 5ns dye laser pulses. The Rydberg atoms are then exposed to an intense single-cycle $\mathrm{THz}$ pulse. The $\mathrm{Na}^{+}$ion or electron yield is measured as a function of the peak $\mathrm{THz}$ field. Ions produced in the interaction region are pushed toward a micro-channel plate (MCP) detector by a small $\simeq 50 \mathrm{~V} / \mathrm{cm}$ extracting step-field applied to the interaction region. The energy distribution of electrons ejected toward the MCP is determined from their TOF.

The two dye lasers, which are pumped at a $15 \mathrm{~Hz}$ repetition rate by the harmonics of an Nd:YAG laser, are collinearly polarized along the spectrometer axis and sequentially excite $3 s$ ground-state atoms, through an intermediate $3 p_{1 / 2}$ level, to the nd Rydberg state of interest. The lasers are focused by $50 \mathrm{~cm}$ lenses and enter the interaction region at right angles. The THz pulse arrives $\sim 20$ ns after the Rydberg excitation.

The intense $\mathrm{THz}$ pulses are generated in $\mathrm{LiNbO}_{3}$ via optical rectification of 150 fs, $790 \mathrm{~nm}, 20 \mathrm{~mJ}$, Ti:Sapphire laser pulses. A tilted-pulse-front-pumping scheme enhances the optical to $\mathrm{THz}$ conversion efficiency [59,69]. The THz intensity is varied using a variable attenuator composed of two wire-grid polarizers. Two off-axis parabolic mirrors are used to focus the THz beam into the interaction region (Fig. 3.4c). The dye laser and $\mathrm{THz}$ beams cross at $\sim 45^{\circ}$ angles and have parallel linear polarizations. The $\approx 2 \mathrm{~mm}$ diameter of the focused $\mathrm{THz}$ beam is much greater than 
that of either dye laser. Thus, the $\mathrm{THz}$ field is uniform over the Rydberg excitation volume.

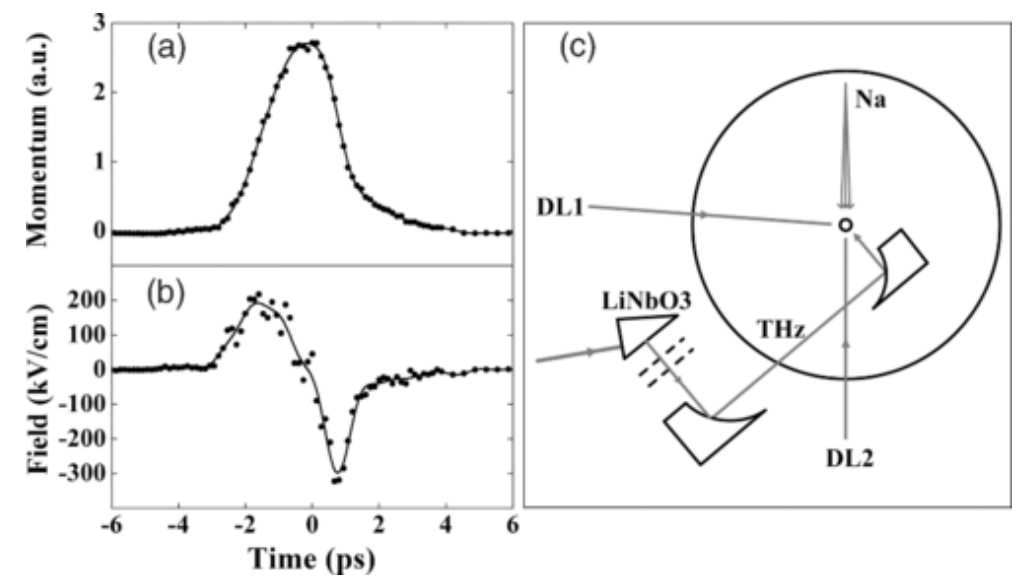

Figure 3.4: a) Maximum momentum transfer (in atomic units) from a $\mathrm{THz}$ field to low-energy photoelectrons as a function of delay between the electron emission and the temporal center of the THz pulse (points) and FFT filtered data (solid line). b) Single-cycle $\mathrm{THz}$ waveform derived from raw data (points) and smoothed data (solid line) in (a). The variation of the data points about the smooth curve reflects the statistical uncertainty which is $\sim 10 \%$ near the extrema. c) Schematic of the $\mathrm{THz}$ ionization experiment. DL1 and DL2 denote dye lasers which drive the $3 s-3 p$ and $3 p-n d$ transitions, respectively. The dashed lines across the $\mathrm{THz}$ beam represent wire-grid polarizers.

Prior to the ionization experiments, we measure the $\mathrm{THz}$ waveform in the interaction region using photoelectron "streaking" [82, 83, 109]. The second dye laser (DL2) is replaced by a 150 fs, $395 \mathrm{~nm}$ frequency-doubled Ti:Sapphire laser pulse, which ionizes atoms in the $3 p_{1 / 2}$ level, creating $\sim 100 \mathrm{meV}$ photoelectrons. The momentum transfer, $\Delta p$, from the $\mathrm{THz}$ field to the photoelectrons, is determined from the electron TOF as a function of the delay, $t_{0}$, between the ionization and $\mathrm{THz}$ pulses. The $45^{\circ}$ crossing angle between the $\mathrm{THz}$ and ionization pulses limits the temporal resolution to $\sim 400$ fs. As shown in Fig. 3.4a, $\Delta p$ can be substantial, with a maximum energy transfer of $100 \mathrm{eV}$.

Relative to the $\mathrm{THz}$ duration, a photoelectron spends an insignificant amount 
of time near its parent ion where the Coulomb field is non-negligible. Thus, the integrated influence of the Coulomb field on the continuum electron is negligible compared to the effect of the strong THz pulse [104]. To an excellent approximation, $\Delta p\left(t_{0}\right)=-\int_{t_{0}}^{\infty} F(t) d t=\alpha\left[A(\infty)-A\left(t_{0}\right)\right]$ where $F(t)$ and $A(t)$ are the THz field and vector potential, respectively, and $\alpha$ is the fine-structure constant. Inspection of Fig. 3.4a shows that $\Delta p=0$ for large negative and large positive delays ${ }^{2}$. Accordingly, without loss of generality, we can define $A(-\infty)=A(\infty)=0$ such that $\Delta p\left(t_{0}\right)=$ $-\alpha A\left(t_{0}\right)$. This last expression is the essence the simpleman's model [22-24] and can be trivially inverted to give the THz field $F(t)=\frac{\mathrm{d}}{\mathrm{d} t}(\Delta p)$.

The THz field (Fig. 3.4b), derived from the momentum transfer data in Fig. 3.4a, appears as a single-cycle sine-like waveform with a pronounced frequency chirp and an amplitude asymmetry between the negative and positive half-cycles. The positive half-cycle has an amplitude (duration) which is $\sim 2 / 3(\sim 3 / 2)$ that of the negative halfcycle. To place the energy transfer in context with other strong-field experiments, we use the average frequency during each half cycle to consistently define, $U_{p} \sim 16 \pm 2$ $\mathrm{eV}$ for both half-cycles of the pulse in Fig. 3.4b.

Beyond determining the $\mathrm{THz}$ field in the interaction region, the streaking measurement enables an examination of energy transfer in the single-cycle limit. Like strong-field ionization of ground state systems, our continuum electrons are "born" near the nucleus with approximately zero kinetic energy. However, unlike a typical ionization experiment, we control the time of ionization within the waveform. We find that two tenets of strong-field energy transfer - i) in the absence of rescattering the maximum energy acquired by an electron in an oscillating field is $\Delta E_{\max }=2 U_{p}$, and ii) an electron ionized at the peak of an oscillating field obtains negligible drift energy - do not apply. Currently, these rules are relied upon to calibrate laser in-

\footnotetext{
${ }^{2}$ This is expected, as the far-field propagating $\mathrm{THz}$ pulse has zero area.
} 
tensities [110-112], distinguish rescattered from directly ionized electrons to enable molecular imaging $[110,113,114]$ or measure the carrier-envelope phase in few-cycle laser pulses $[115,116]$, or to interpret dynamics relevant to a variety of strong-field processes [112, 117-120]. However, they fail completely in the single-cycle limit due to the breakdown of the slowly varying envelope approximation.

In a many-cycle pulse, the extrema of the vector potential $\left|A_{\max }\right|=F_{\max } / \omega$ occur at the zeros of the electric field, and $A=0$ at the field extrema. However, for a single-cycle sine-wave, $\left|A_{\max }\right|=2 F_{\max } / \omega$ at the zero-crossing and $|A|=F_{\max } / \omega$ at the field extrema. Accordingly, $\Delta E=8 U_{p}$ and $2 U_{p}$ for ionization at the central zerocrossing and field maxima, respectively. The momentum transfer data in Fig. 3.4a support these predictions, with a maximum energy transfer of $(6.2 \pm 1.2) U_{p}$ at the zero-crossing and $\sim 2 U_{p}$ at the field extrema.

We have used the single-cycle pulses to measure the ionization yield, as a function of $F_{\max }$, for a range of states with $n \geq 5$. No ionization from the $5 d$ level was observed. When the $50 \mathrm{~V} / \mathrm{cm}$ clearing field is replaced with a $7 \mathrm{kV} / \mathrm{cm}$ field-ionization ramp, no population transfer to bound-states with $n>15$ is observed for any initial state or THz field strength, indicating purely adiabatic ionization. For $n \geq 10$, the ionization yield saturates for large $\mathrm{THz}$ fields. Assigning unit ionization probability to this saturated signal provides the ionization yield to probability calibration.

Fig. 3.5 shows representative ionization probability vs. $F_{\max }$ curves for $6 \leq n \leq 15$. From these data we define $F_{10 \%}$ as the peak THz field that produces $10 \%$ ionization for a given $n$ state. Measured values of $F_{10 \%}$ vs. $n$ are plotted in Fig. 3.6. Notably, the measured thresholds differ significantly from those predicted for adiabatic OTBI, exhibiting a novel $n^{-3}$ dependence rather than the expected $n^{-4}$ scaling. The $n^{-3}$ scaling reflects a suppression of adiabatic ionization for $n>6$ due to the short period/duration of the $\mathrm{THz}$ field relative to atomic time-scales distinct from $\tau_{K}$. 


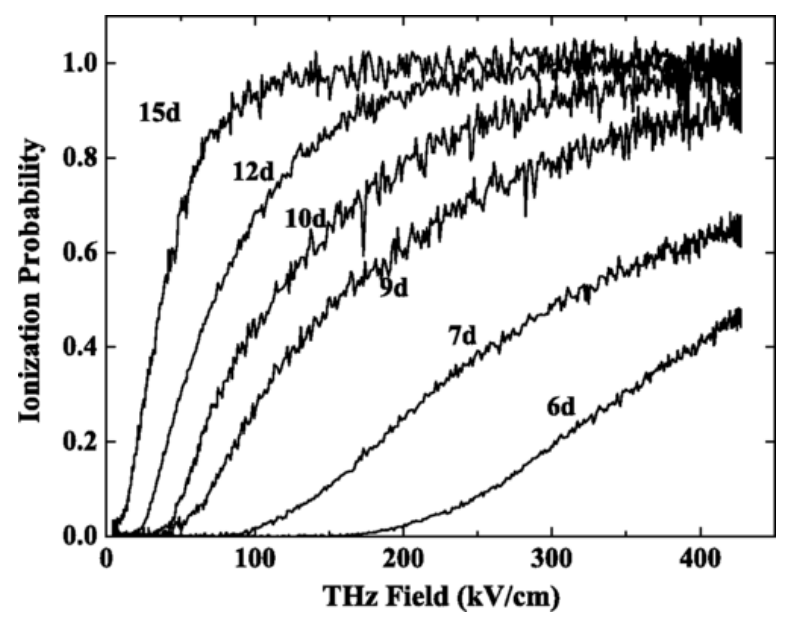

Figure 3.5: $\quad$ Measured ionization probability as a function of $F_{\max }$ for several $n d$ states (see Appendix I for the calibration of unsaturated ionizations). The $\mathrm{THz}$ field is calibrated using THz streaking measurements (Fig. 3.4).

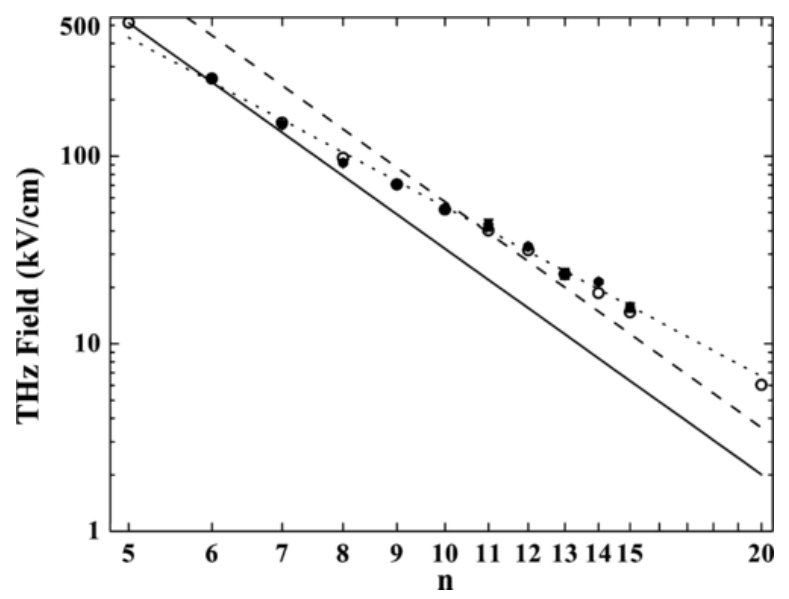

Figure 3.6: Peak THz field required for $10 \%$ ionization as a function $n$. (•) experimental data; (o) CTMC calculation; (dotted line) best fit, $n^{-3} / 96$, to experimental data; predicted adiabatic ionization thresholds $\mathcal{F}_{H}=n^{-4} / 9$ (dashed line) and $\mathcal{F}_{0}=n^{-4} / 16$ (solid line) for hydrogenic and non-hydrogenic Rydberg atoms, respectively. The experimental fields have been scaled by $1.05 \times$ to obtain the best agreement with the calculation. Experimental error bars are comparable to the size of the data symbols and are not visible for all points.

Adiabatic OTBI is energetically allowed provided $F>E^{2} / 4$, where $E$ is the electron's energy in the field. For ground-state atoms, the electron suffers only a small second-order Stark shift in the field, so $E^{2} / 4 \simeq n^{-4} / 16 \equiv \mathcal{F}_{0}$. In classical 
terms, OTBI can proceed provided the electron has sufficient time to encounter and pass over the barrier while $F>\mathcal{F}_{0}$. For ground-state atoms this time is $<\tau_{K}$.

The situation is more complicated for Rydberg states [10]. Upon the application of our $\mathrm{THz}$ field, the $n d$-states are projected onto a manifold of Stark states which have different dipole orientations and accompanying linear Stark shifts. For hydrogenic atoms, the saddle-point at threshold is only accessible to the most "downhill" Stark state. When the negative energy shift of this state is taken into account, its OTB ionization threshold $\mathcal{F}_{H}=n^{-4} / 9$, defines the minimum field at which ionization can occur. Due to their orientation, the other Stark states have negligible overlap with the saddle when $F=\mathcal{F}_{H}$. They require a larger "hole" in the binding potential and, accordingly, a larger field to ionize [121].

In non-hydrogenic atoms like Na, Stark-states are coupled by the non-Coulombic potential, and Landau-Zener transitions between states with different orientations can occur as the field rises from zero to its maximum [10, 93]. Classically, a bound electron can scatter from one Stark-trajectory to another each time the electron passes near the non-hydrogenic ion core. If the scattering rate is sufficiently high compared to the slew rate of the field, the electron can have zero net Stark shift during the rise of the field. Therefore, it can ionize at (or near) $\mathcal{F}_{0}$, as predicted for atoms with no Stark shift [91].

For the lowest $n$-states studied, the thresholds approach $\mathcal{F}_{0}=n^{-4} / 16$, indicating non-hydrogenic OTBI and a core-scattering rate larger than the slew rate of the $\mathrm{THz}$ field. For higher $n$, the core-scattering rate decreases and the ionization thresholds rise above $\mathcal{F}_{0}$. This is consistent with the transition from non-hydrogen-like to hydrogenlike OTBI observed previously for ramped-field ionization [93]. In the presence of this effect alone, we would expect a gradual transition from thresholds scaling as $\mathcal{F}_{0}$ at low $n$ to $\mathcal{F}_{H}$ at high $n$. Interestingly, for the highest $n$ states studied, the ionization 
thresholds significantly exceed $\mathcal{F}_{H}=n^{-4} / 9$. The additional ionization suppression is caused by the extended time required for higher $n$ electrons to traverse the saddlepoint in the field-dressed potential.

For $F_{\text {max }} \simeq \mathcal{F}_{0}$, the outer turning point of the downhill orbit is located near the saddle-point at a radius, $R_{0}=4 n^{2}$. Thus, the orbit is nearly twice as large as in zero field. For the states studied, the extra distance the electron must cover, accompanied with its extremely low radial velocity between $r=2 n^{2}$ and $r=R_{0}$, leads to a nearly four-fold increase in the orbit period (140 fs and 2.3 ps for $n=6$ and $n=15$, respectively). Provided $F_{\max }$ is above threshold, ionization is energetically allowed but can only occur if the electron has sufficient time to traverse the saddle. For $n>6$ this dynamical criterion can be satisfied by increasing $F_{\max }$ which in turn i) increases the interval during which ionization is energetically allowed, ii) increases the radial velocity of electrons traveling towards the saddle; and iii) moves the saddle-point to smaller $r$.

The preceding arguments are fully supported by a classical trajectory Monte Carlo (CTMC) simulation. The calculation (details in Ref. [99]), assumes an asymmetric field like that shown in Fig. 3.4b, and a soft Coulomb potential $V=-1 / \sqrt{r^{2}+r_{0}^{2}}$ to simulate the non-hydrogenic core. Larger $r_{0}$ values result in a greater rate of scattering between Stark states. The computed threshold for $n=15$ is found to be independent of physically reasonable values of $r_{0}$ and agrees with the measured value within $5 \%$. With the field calibration fixed, we adjust $r_{0}$ to give the best overall agreement between the simulated and experimental thresholds (Fig. 3.6). The optimum value $r_{0}=0.03$ is not critical and reasonable agreement is obtained for $0.01 \leq r_{0} \leq 0.1$. Calculations for $n=20$ and $n=5$ confirm the further growth of the suppression at higher $n$ and its disappearance at low $n$, respectively.

Fig. 3.7 shows the electron energy distributions produced during the ionization of 
several $n$ states for $F_{\max }=430 \mathrm{kV} / \mathrm{cm}$. Counter to expectations for multi-photon or OTBI in a multi-cycle field, electrons with the highest energies are produced from states which are more tightly bound. Perturbatively, for a given multi-photon order, higher-energy electrons would be produced from the most weakly-bound states. For multi-cycle OTBI ionization, the most tightly-bound states ionize near the peak of the waveform where the vector potential is small, resulting in reduced momentum transfer as compared to more weakly bound states which can ionize closer to the zero-crossing of the field.

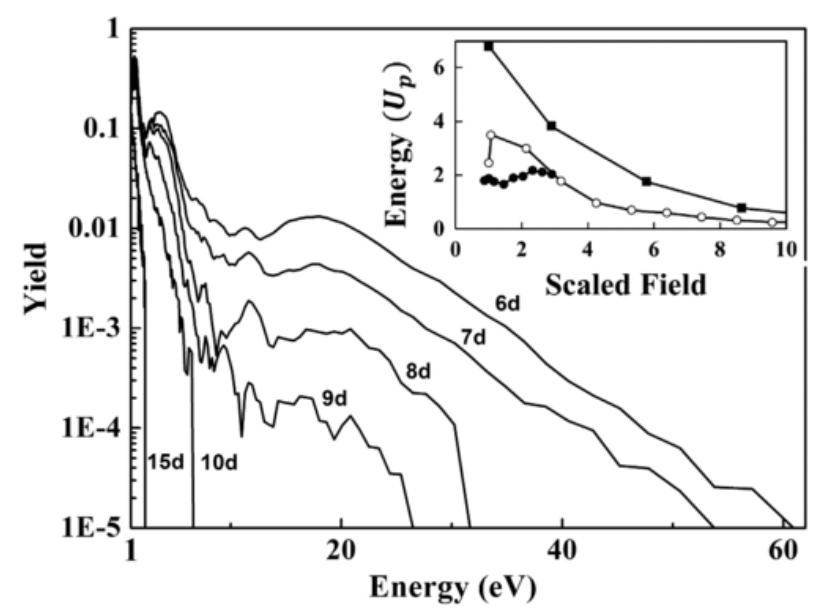

Figure 3.7: Electron energy distributions (energy-integrated yields normalized to one) for different $n$-states ionized by a $430 \mathrm{kV} / \mathrm{cm}$ single-cycle $\mathrm{THz}$ field. Inset: Maximum electron energy as a function of $F_{\max }$ for representative states, n=7 $(\bullet)$, $\mathrm{n}=11(\circ)$ and $\mathrm{n}=15(\mathbf{\square})$. Error bars are smaller than the data symbols.

As discussed previously, the situation is different for single-cycle pulses. An electron ionized at a field-maximum in a single-cycle sine wave acquires an energy $\Delta E=2 U_{p}$. In the same field, an electron in a more weakly-bound level will ionize on the rising edge of the first half-cycle, and ultimately gain less energy from the field. Accordingly, in units of $U_{p}$, the maximum energy $\Delta E_{\max }$ transferred to an electron from a given $n$ state should decrease as $F_{\max }$ is raised from near, to well above, $F_{10 \%}$.

The Fig. 3.7inset shows $\Delta E_{\max }$ (scaled to $U_{p}$ ) as a function of $F_{\max }$ (scaled to 
$\left.F_{10 \%}\right)$ for $n=7,11$, and 15 . The data shown represent the evolution of analogous results from low to high $n$. For low $n$, with $F_{\max } \simeq F_{10 \%}$, ionization occurs near, or slightly after, the peak in the second half-cycle such that $\Delta E_{\max } \sim 2 U_{p}$. Since $F_{\max }$ cannot be increased sufficiently to saturate the ionization probability on the rising edge of the pulse, no significant decrease in $\Delta E_{\max }$ is observed for the lowest $n$ states. For intermediate $n, \Delta E_{\max } \simeq 2 U_{p}$ for $F_{\max } \simeq F_{10 \%}$, as expected for ionization near the peak of the second half-cycle in the field. $\Delta E_{\max }$ grows for a modest increase in $F_{\max }$ above $F_{10 \%}$, likely due to the greater probability of ionization between the two field maximum, i.e. slightly after (before) the first (second) half-cycle. For $F_{\max } \gg F_{10 \%}$, $\Delta E_{\max }$ progressively falls as ionization occurs ever earlier on the rising-edge of the first half-cycle. For high $n$, energies well above $2 U_{p}$ are observed for fields near, to moderately above, threshold. We suspect that this is due to the breakdown of the adiabatic approximation. Electrons ionizing from these states need not reverse direction as they move in their extended orbits over a significant portion of each half cycle of the field. Accordingly, they can acquire non-negligible energy and are effectively "born" before passing over the saddle. This enables them to gain a large kinetic energy from the field, in spite of the fact that they may not actually traverse the saddle until after the peak of the pulse.

In summary, we have explored strong-field ionization of excited atoms using singlecycle $\mathrm{THz}$ pulses. We find that adiabatic over-the-barrier ionization is suppressed due to the extended times required for Rydberg electrons to leave the binding potential, resulting in a novel $n^{-3}$ ionization-field scaling. A similar suppression might influence strong-field ionization of delocalized electrons from large molecules. We have also shown that, in contrast to ionization in multi-cycle fields, electrons that are ionized near field-extrema acquire substantial energies $\left(\sim 2 U_{p}\right)$, and those ionized near the zero-crossing can obtain much larger energies $\left(>6 U_{p}\right)$, even in the absence 
of rescattering. As experimentally available optical pulses approach the single-cycle limit, the physics underlying our results will impact the interpretation and feasibility of measurements involving ground-state atoms and molecules, particularly those where distinguishing recollision from directly ionized electrons is important, or where mapping electron energy to ionization time is desired.

\section{A theoretical extension to the short pulse regime}

Soon after our experimental observation and explanation of the transition from an $n^{-4}$ to an $n^{-3}$ threshold scaling behavior for strong-field ionization of low-lying Rydberg states by single-cycle THz pulses, Yang and Robicheaux theoretically extend our work to high-lying Rydberg states, or in other words, to the short-pulse regime where the scaled frequency $\Omega \ll 1$ [122]. Surprisingly, in this case, the required threshold field amplitude is found to scale inversely with the binding energy and, thus more weakly bound electrons require larger fields for ionization ${ }^{3}$. The dominant ionization mechanism is identified as a nonzero spatial displacement of the electron: By interacting with a very short single-cycle pulse, the ionization can only occur during a very short fraction of one Rydberg period, during which the electron experiences a sudden spatial displacement $\Delta r$ with approximately no momentum or energy transfer from the field. The condition for ionization can be estimated as [122],

$$
E_{n}+\frac{1}{r_{n}}-\frac{1}{r_{n}+\Delta r} \geq 0
$$

given the energy $E_{n}=-\frac{1}{2 n^{2}}$ and the Bohr orbital radius $r_{n}=n^{2}$ of the electron, Eq. 3.13 requires $\Delta r \geq n^{2}$. The displacement can be estimated from the motion of a free electron driven by a single-cycle pulse, and the exact value depends on the

\footnotetext{
${ }^{3}$ This result is related to stabilization of ionization in strong high-frequency fields as noted in Chap. 1.
} 
pulse shape, but generally follows $\Delta r \propto F_{m} t_{w}^{2}$, where $F_{m}$ and $t_{w}$ are the maximum amplitude and duration of the pulse, respectively. As a result, the threshold ionization threshold field scales as [122],

$$
F \propto \frac{n^{2}}{t_{w}^{2}}
$$

Yang and Robicheaux gave an empirical formula predicting the threshold behavior over the entire range of the scaled frequencies, from the long-pulse to the short-pulse limit [122],

$$
F n^{4}=\frac{1}{9} e^{-\Omega}+\frac{1}{10} \Omega^{1 / 3} e^{-(\Omega-1)^{2}}+\frac{1}{2 \pi} \Omega^{2} e^{-1 / \Omega}
$$

The empirical expression in Eq. 3.15 is a simple combination of different threshold behaviors in three scaled-frequency regimes: the first term corresponds to the long pulse limit, the second term falls in the regime where the time effects (the extended time required for Rydberg electrons to traverse the saddle-point) becomes noticeable, and the last term corresponds to the short pulse limit. The exponential factors in Eq. 3.15 "turn on" the appropriate threshold scaling regimes.

Yang and Robicheaux uncovered additional features of single-cycle ionization. First, they noted the formation formation of an "ionization window" for ionization of a series of Rydberg states by a specific single-cycle pulse [122]. The window appears as ionization "turns off" at both low $n$ and high $n$ states because of the different threshold scaling behaviors at low $n$ (in the long-pulse adiabatic-ionization regime $F \propto n^{-4}$ ) and high $n$ (in the short-pulse displacement-ionization regime $F \propto n^{2}$ ). Second, they discovered "ripple" structures in the ionization-probability curves as a function of the pulse duration and the field amplitude. These are a manifestation of the competition between the atomic Coulomb attraction and the spatial displacement induced by a single-cycle pulse [123]. Third, they predicted an asymmetry of electron-emission along the field polarization direction (left-right asymmetry) [123]. 
The ionized electron is preferably emitted to the positive- $z$ direction (right side), which is consistent with our qualitative picture of the ionization process and our experimentally observed electron energy spectra.

Note that the transitions between ionization regimes, as well as the displacement and the ionization suppression effects have been experimentally studied for ionization induced by half-cycle pulses [100]. It would be interesting to experimentally extend the single-cycle pulse induced ionization to the short-pulse regime in the future.

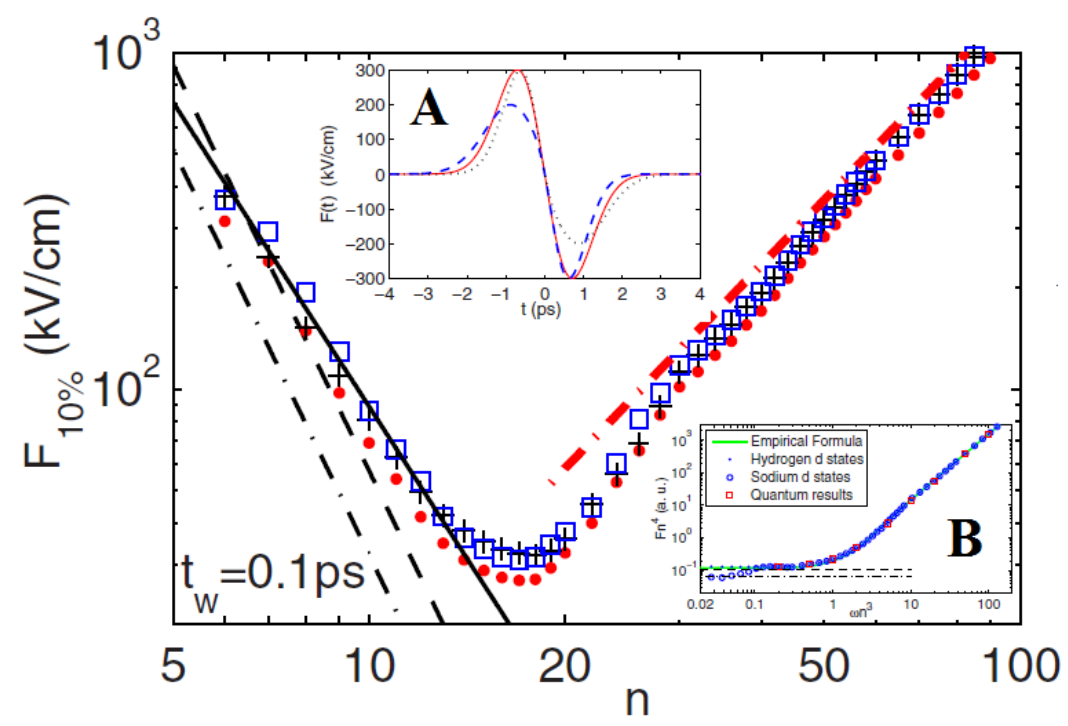

Figure 3.8: 3D CTMC simulations of threshold scaling behaviors for field ionization of sodium by single-cycle THz pulses, from Ref. [122]. Red solid points (symmetric pulse), open squares (asymmetric pulse), "+" (inverted asymmetric pulse). $F \propto n^{-3}$ (black solid line), $F=1 /\left(9 n^{4}\right)$ (black dashed line), $F=1 /\left(16 n^{4}\right)$ (black dot-dashed line), $F=\sqrt{\frac{2}{\pi e}} \frac{n^{2}}{t_{w}^{2}}$ (red dot-dashed line). Inset A) Three typical single-cycle waveforms. The solid, dashed, and dotted curves represent, respectively, the "symmetric", "asymmetric", and "inverted asymmetric" field profiles given by the vector potential $A(t)=-\frac{F_{m} t_{w}}{C_{0}} e^{\left[-1 \pm \frac{1}{a} \tanh \frac{b t}{t_{w}}\right]\left(\frac{t}{t_{w}}\right)^{2}}$, where $F_{m}$ and $t_{w}$ are the maximum pulse amplitude and the defined pulse duration, respectively ( $4 t_{w}$ is approximately the period of the pulse); $a$ and $b$ are two adjustable parameters determining the pulse asymmetry, and $C_{0}$ is dependent on the values of $a$ and $b$. Inset B) Threshold scaling behaviors shown in the scaled field $\left(F n^{4}\right)$ and scaled frequency $\left(\omega n^{3}\right)$ coordinates. A "symmetric" pulse with $t_{w}=1 \mathrm{ps}$ is used. The quantum results are for hydrogen $15 \mathrm{~d}$ state at different scaled frequencies. The dashed and the dot-dashed lines represent the thresholds for hydrogen and sodium in the low-frequency limit, respectively. 


\title{
Chapter 4
}

\section{Ionization of Oriented Atoms by}

\section{Intense, Single-Cycle THz Pulses}

\begin{abstract}
We have employed intense, single-cycle $\mathrm{THz}$ pulses to explore the ionization of atomic Rydberg-Stark states as a function of the magnitude and direction of their permanent dipole moments. The presence of a permanent dipole moment can substantially influence strong field ionization dynamics in atoms and molecules and lead to directional asymmetries in electron emission. In the experiments, atoms are laser excited to Rydberg-Stark states with well-defined permanent dipole moments in the presence of a small static electric field, and then exposed to and ionized by a single-cycle $\mathrm{THz}$ pulse. The peak $\mathrm{THz}$ field strength required for ionization shows a pronounced variation among states with different dipole moments but with nearly identical binding energies. This orientation dependence can be attributed to diabatic population transfer between "uphill" and "downhill" oriented states and an asymmetry in the peak $\mathrm{THz}$ field amplitude in the forward and backward directions.
\end{abstract}


In Chap. 3, we studied the ionization of excited atoms by intense single-cycle $\mathrm{THz}$ pulses. A transition of the strong-field over-the-barrier ionization threshold scaling from $F \propto n^{-4}$ to $F \propto n^{-3}$ was observed, reflecting the suppression of corescattering during the ionization pulse and the extended times required for Rydberg electrons to escape over the field-dressed Coulomb potential barrier [124]. In this chapter, we explore the ionization of oriented atoms by intense, single-cycle $\mathrm{THz}$ pulses. Here "orientation" refers to the establishment of a permanent electric dipole moment within the atom in a specific direction in the lab frame [125]. The presence of a permanent dipole moment can substantially influence strong field ionization dynamics in atoms and lead to directional asymmetries in electron emission. Measurements of this orientation dependence, beyond uncovering the ionization dynamics, play an important role in understanding or optimizing strong-field processes following ionization, e.g., electron rescattering that enables high harmonic generation and/or non-sequential double ionization. In addition, the atomic study provides a relevant analog for molecular systems, as most molecules are polar and their interactions with laser fields depend on the molecular orientation [126].

The atomic orientation is achieved by making use of DC Stark effect. A static electric field $F$ (directed along $+z$ ) breaks the spherical symmetry of the atoms. As a result, the Rydberg-Stark eigenstates exhibit permanent electric dipole moments $\mu=$ $-\langle z\rangle$, and the corresponding eigenenergies experience linear shifts, a.k.a. Stark shifts, $\Delta E=F\langle z\rangle$, to the lowest order in perturbation theory [127]. In the experiments, the applied static fields are significantly smaller than $F=1 / 3 n^{5}$. i.e., the field strengths are significantly smaller than those required for classical field ionization and the Stark energy shifts are significantly smaller than the spacings between adjacent $n$ states. Therefore, the major effects of the static electric field is to orientate the RydbergStark trajectories relative to the $z$ axis, as well as to provide small energy shifts that 
facilitate single-state laser excitations [127].

In the experiments, two tunable, 5 -ns pulsed dye lasers excite ground state $\left(3 s_{1 / 2}\right)$ $\mathrm{Na}$ atoms in a thermal beam in vacuum through the $3 p_{1 / 2}$ state to the Rydberg-Stark state of interest in the $n$-manifold. The atoms are then exposed to and ionized by an intense single-cycle $\mathrm{THz}$ pulse. The $\mathrm{Na}^{+}$ion or electron yield is measured as a function of the peak $\mathrm{THz}$ field.

\section{a}

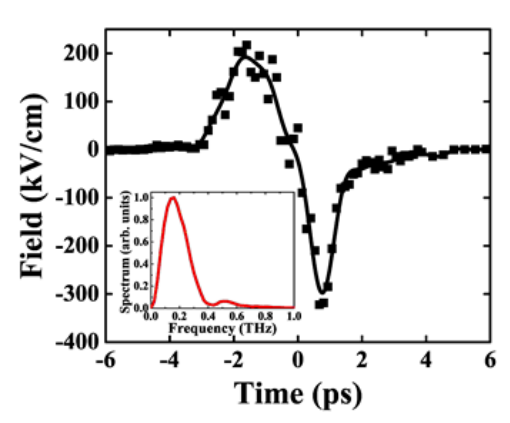

b

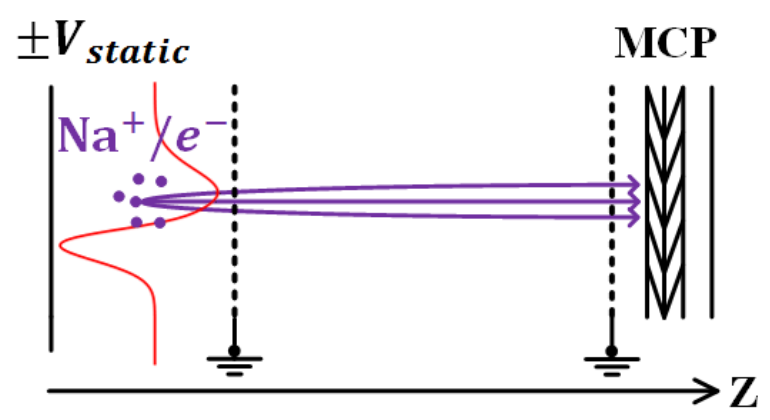

c

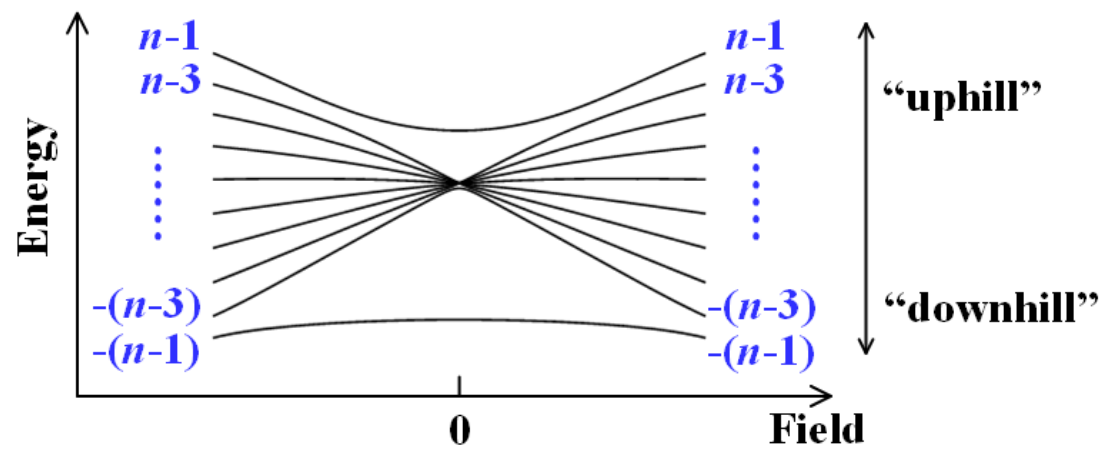

Figure 4.1: a) THz waveform obtained form photoelectron streaking measurements. b) Schematic of the interaction region. The dye lasers, the THz field and the static electric field are collinearly polarized along the spectrometer axis. The sign of the static field can be changed to produce opposite polarization of the static field relative to that of the $\mathrm{THz}$ field. Ions/electrons are collected when the applied static field is positive/negative. c) Schematic of a Na Stark manifold associated with a particular principal quantum number, $n$.

The dye lasers, the $\mathrm{THz}$ beam and the Na beam intersect at the center of a time- 
of-flight (TOF) spectrometer between two capacitor plates that provide the small static field in the interaction region. The polarization axes of the dye lasers, the $\mathrm{THz}$ pulse and the static field are along the spectrometer axis, defining the $z$-axis. The polarity of the THz field is fixed, whereas the sign of the static field can be changed to produce opposite polarization of the static field with respect to that of the $\mathrm{THz}$ field (see Fig. 4.1b).

The intense $\mathrm{THz}$ pulses are generated in $\mathrm{LiNbO}_{3}$ via optical rectification of 150 fs, $790 \mathrm{~nm}, 20 \mathrm{~mJ}$, Ti:Sapphire laser pulses with a tilted-pulse-front-pumping phasematching scheme [59, 69]. Photoelectron "streaking" [82, 83, 109] measurements show that the $\mathrm{THz}$ field at focus in the interaction region has a single-cycle, sine-like waveform with a pronounced frequency chirp and a peak field amplitude asymmetry of $\sim 2 / 3$ between the positive and negative half-cycles (see Fig. 4.1a).

For more detail about the experimental realization and $\mathrm{THz}$ characterization, see Chap. 3.

For clarification, in the following discussion, we will use the approximate parabolic quantum number " $k$ " to order the Stark states in an $n$-manifold (see Fig. 4.1c). Each $k$-state is adiabatically connected to a zero field spherical $n l$ state. In $\mathrm{Na}$,

$$
\begin{aligned}
(n+1) s & \rightarrow k=-(n-1) \\
n l & \rightarrow k=-(n-1)+2(l-1) \\
(n+1) p & \rightarrow k=n-1
\end{aligned}
$$

The $k=(n-1)$ state has the most positive Stark shift (i.e. the extreme "uphill" or blue Stark state), whereas the $k=-(n-1)$ state exhibits the most negative Stark shift (i.e. the extreme "downhill" or red Stark states).

Fig. 4.2 compares the ionization probability as a function of peak $\mathrm{THz}$ field strength for states initially prepared in the "uphill" and "downhill" Stark levels 
$|k|=n-3$ in the $n=10,11$ and 12 manifolds, with static fields directed in the $+z$ and $-z$ directions. As shown, the downhill state is more easily ionized by the single-cycle THz field when the static field is directed in the $-z$ direction, whereas ionization of the uphill state is not sensitive to the polarization of the static field relative to that of the $\mathrm{THz}$ field.
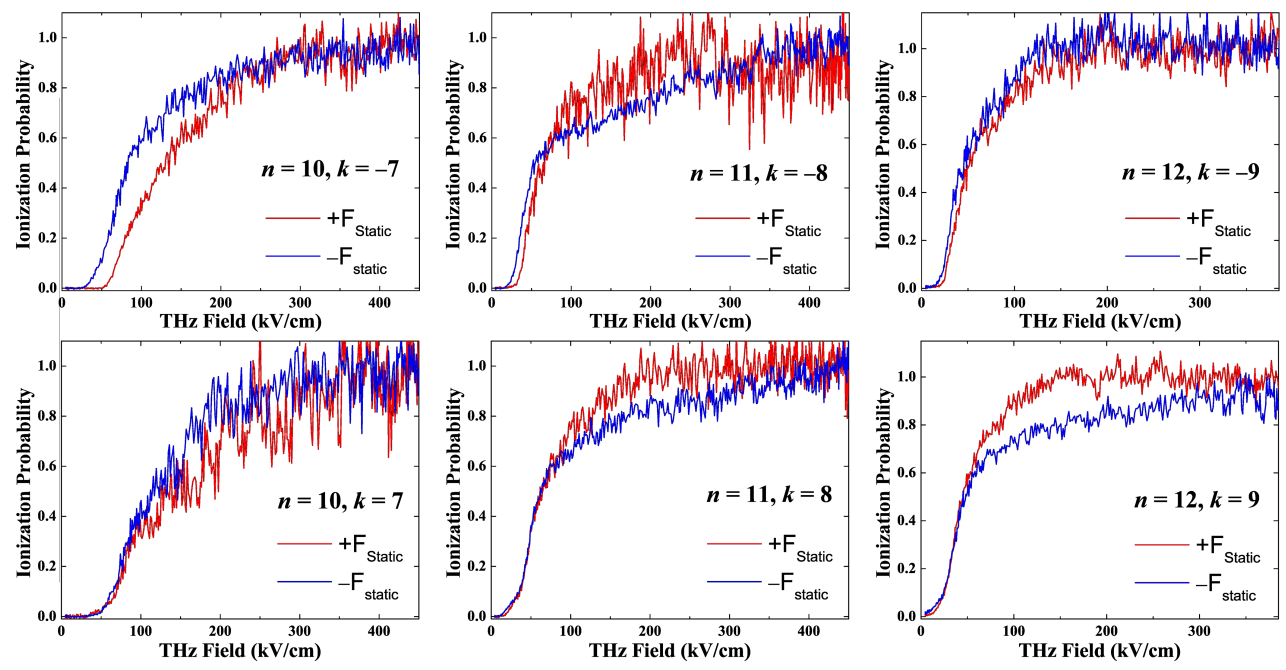

Figure 4.2: Measured ionization probability as a function of peak $\mathrm{THz}$ field strength for "uphill" and "downhill" Stark states.
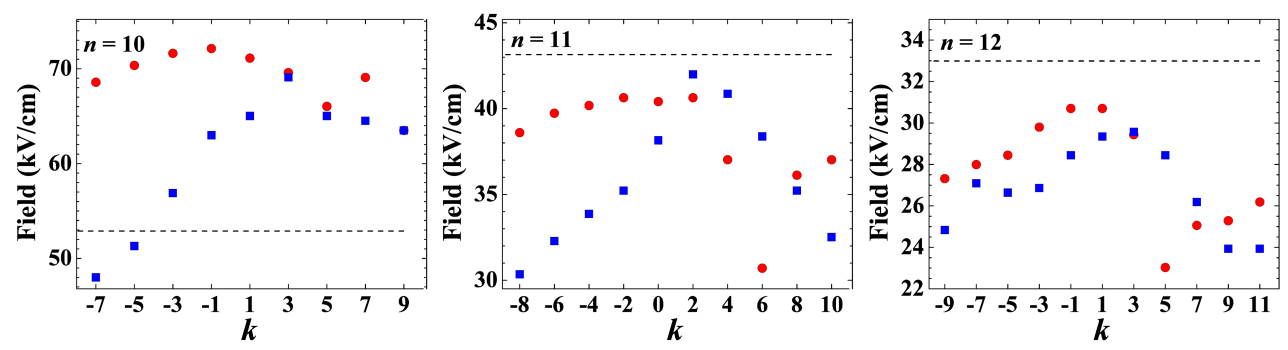

Figure 4.3: Peak THz field required for $10 \%$ ionization as a function of $k$ within different $n$-manifolds, $n=10,11$ and 12. (red circles) $F_{\text {static }}>0$; (blue squares) $F_{\text {static }}<0$; (dashed line) measured ionization threshold for the $n d$ state in zero static field.

Fig. 4.3 plots the ionization threshold fields (defined as the peak $\mathrm{THz}$ field that produces $10 \%$ ionization) as a function of the approximate parabolic number $k$ for 
the $n=10,11$ and 12 Stark manifolds. The peak $\mathrm{THz}$ field strength required for ionization shows a pronounced variation among Stark states whose permanent dipole moments differ in amplitude and/or direction.

As studied in Chap. 3, our experiments of strong-field ionization of low-lying Rydberg states by single-cycle $\mathrm{THz}$ pulses, fall in the long-pulse/low-frequency regime and over-the-barrier (OTB) ionization is expected. The pulsed field, OTB ionization of non-hydrogenic Rydberg atoms with non-zero quantum defect can follow either adiabatic or diabatic pathway, and the process depends on the core-scattering rate during the ionization pulse relative to the slew rate of the pulse. In the experiments performed in Chap. 3, we have observed the transition from non-Hydrogen-like adiabatic to Hydrogen-like diabatic ionization behavior. The experiments showed that ionization of extremely low $n$ states $(n \leq 6)$ by our THz fields follows the adiabatic pathway, indicating a core-scattering rate larger than the slew rate of the THz field. And ionization of higher $n$ states $(n \geq 10)$ follows the diabatic pathways, indicating a suppressed core-scattering rate. Therefore, it is evident that for the Rydberg-Stark levels studied in experiments described in this Chapter, $n=10-12$, hydrogen-like diabatic OTB ionization is expected.

The diabatic ionization can be further confirmed from the following calculation. Consider a Stark state exposed to a pulsed ionization field. If we track the evolution of its energy as the field rises, a diabatic ionization pathway is the one for which all the (non-zero-field) avoided energy crossings are traversed diabatically. This requires the slew rate of the $\mathrm{THz}$ field to be much higher than a critical slew rate, $S_{c}{ }^{1}$ for a given avoided crossing, for which the avoided crossing is traversed rapidly compared

\footnotetext{
${ }^{1}$ The ratio of the slew rate of the field $S_{\text {field }}$ to the critical slew rate of the avoided crossing $S_{c}$ characterizes the adiabaticity of the transition, if $S_{\text {field }} \gg S_{c}$, a pure diabatic transition occurs whereas the transition is adiabatic for $S_{\text {field }} \ll S_{c}$. For more details about the critical slew rate, see Appendix H.
} 
to $1 / \Delta E_{0}$, where $\Delta E_{0}$ is the size of the avoided crossing. The critical slew rate can be estimated by assuming an isolated two-level $\left(E_{1}\right.$ and $\left.E_{2}\right)$ Landau-Zener transition $[10,128-130]$

$$
S_{c}=\left(\Delta E_{0}\right)^{2} /\left(\frac{\mathrm{d} E_{1}}{\mathrm{~d} F}-\frac{\mathrm{d} E_{2}}{\mathrm{~d} F}\right)
$$

The size of the avoided crossing bwtween two Stark states in an $n, m=0$ manifold is approximately $\Delta E_{0} \simeq \delta_{l=0}^{\prime} / n^{4}[10]$ where $\delta_{l=0}^{\prime}$ is the absolute value of the quantum defect $\delta_{l=0}$. For Na $\delta_{l=0} \simeq 1.35$ and $\delta_{l=0}^{\prime} \simeq 0.35$. If we use the smallest possible value of $\left(\frac{\mathrm{d} E_{1}}{\mathrm{~d} F}-\frac{\mathrm{d} E_{2}}{\mathrm{~d} F}\right) \simeq 3 n$ between two adjacent Stark levels, the critical slew rates are calculated to be $S_{c} \simeq 0.35^{2} /\left(3 n^{9}\right) \simeq(8.7,3.7,1.7) \mathrm{kV} \cdot \mathrm{cm}^{-1} \cdot \mathrm{ps}^{-1}$ for $n=(10,11,12)$, respectively. The ionization threshold for the extreme downhill Stark state in an $n$ manifold is $F_{0} \simeq 1 /\left(96 n^{3}\right)^{2}$, and are $F_{0} \simeq(54,40,31) \mathrm{kV} \cdot \mathrm{cm}^{-1}$ for $n=(10,11,12)$, respectively. The $\mathrm{THz}$ pulse has a rise time of $\sim 1 \mathrm{ps}$. Therefore, the slew rate of the THz field far exceeds the critical slew rates, validating the assumption of diabatic ionization.

One important feature of diabatic OTB ionization is that an "uphill" state is substantially harder to ionize than a "downhill" state and the ionization threshold increases monotonically with increasing $k$ within one $n$-manifold. The variation of ionization threshold arises from differences in orientation of the atomic wavefunctions with respect to the direction of the saddle point created by the ionization field, as well as the Stark energy shifts. The extreme "uphill" / "downhill" state has the least/most overlap with the saddle point, and is the hardest/easiest to ionize. So it would not be surprising to observe orientation-dependent ionization for a uni-directional ionizing field. However, in our experiments, the ionization threshold does not increase monotonically as $k$ increases.

\footnotetext{
${ }^{2}$ this is higher than $F_{0} \simeq 1 /\left(9 n^{4}\right)$, due to the extended times required for Rydberg electrons to escape over the field-dressed Coulomb potential barrier, as discussed in Chap. 3.
} 
For a single-cycle $\mathrm{THz}$ field, and for different static field directions, the ionization dynamics are complicated by (multi-level) Landau-Zener transitions at zero-field avoided crossings, as these result in population transfer between "uphill" and "downhill" oriented states. This "uphill" $\leftrightarrow$ "downhill" transfer occurs when the system diabatically traverses the zero-field avoided crossings. Although the slopes of the field-dependent eigenenergies remain (approximately) the same, the net field changes sign and the saddle point in the combined Coulomb-Stark potential moves to the other side of the atom. Due to this "uphill" - "downhill" inversion, it is important to clarify the statement that "a 'downhill' state is more easily ionized than an 'uphill' state". Specifically, an orientated Rydberg-Stark atom is more easily ionized when its instantaneous dipole moment is aligned along the instantaneous ionizing field. In our experiments, depending on the electronic orientation ("uphill" or "downhill") of the state at field extrema, ionization will preferentially occur at the peak of the positive or negative half-cycle of the $\mathrm{THz}$ field.

Fig. 4.4 shows the calculated ionization threshold fields of the Stark states in a single $n$-manifold, based on the following assumptions. First, the extreme "downhill" state ionizes at the lowest field $F_{0}$, the ionization thresholds increase linearly with $k$, and the extreme "uphill" state ionizes at a field a factor of 2 higher than that of the extreme "downhill" state (i.e., $F_{n k}=\frac{k}{2(n-1)} F_{0}+\frac{3}{2} F_{0}$ ). Second, the slew rate of the THz field is sufficiently high that the Landau-Zener transition at the zero-field avoid crossings is purely diabatic, i.e., the uphill-downhill inversion occurs with unit probability and the sign of $k$ changes as the system traverses through zero-field avoidcrossings. Third, the peak field amplitude asymmetry is $\sim 2 / 3$ between the positive and negative half-cycles of the $\mathrm{THz}$ waveform, and ionization occurs at the field extrema at which the field strength first exceeds the ionization threshold. However, for consistency with the experiments, if ionization occurs at the lower, peak of the 
positive half-cycle, the ionization threshold is still given in terms of the peak field, i.e. the peak amplitude of the negative half-cycle.
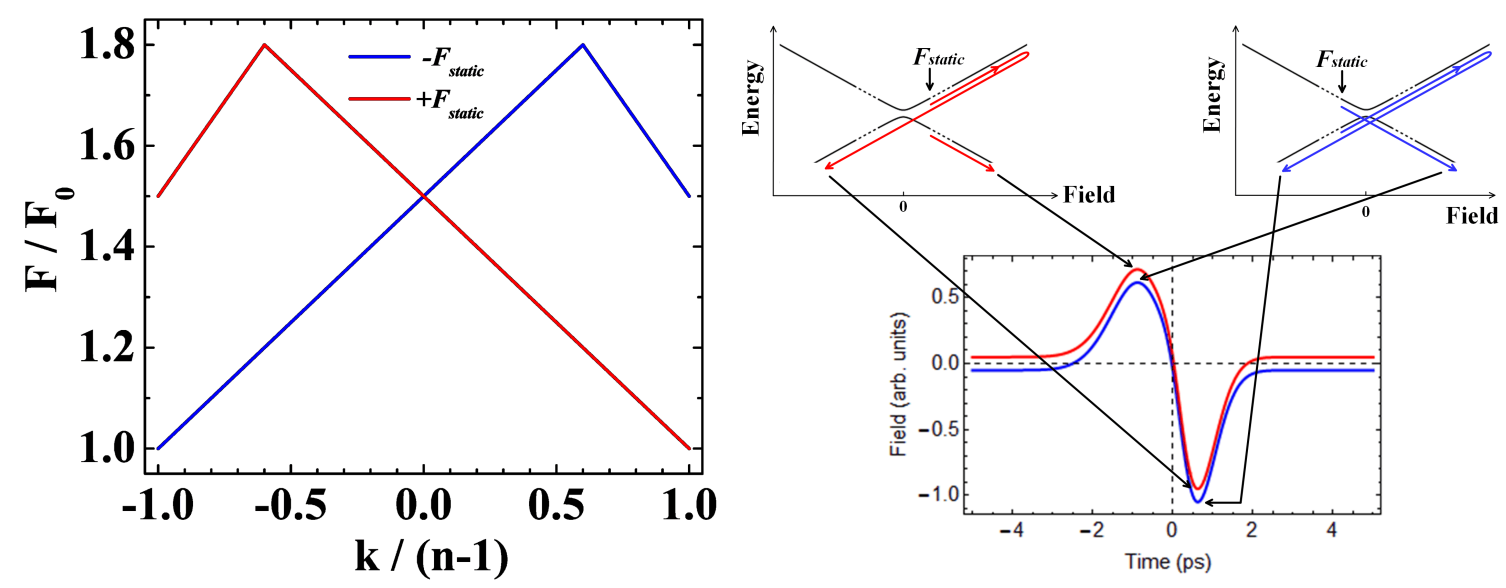

Figure 4.4: Dynamics of single-cycle ionization of oriented Rydberg-Stark states. Left) Ionization threshold field as a function of $k$, the approximate parabolic number, in an $n$-manifold. Blue and red curves are with negative and positive static fields applied, respectively. Right) the combined $\mathrm{THz}$ and static fields waveform and ionization pathways for the extreme Stark levels, (blue) negative static field; (red) positive static field.

The calculations agree qualitatively with our experimental observations. The results indicate that an "uphill" state may be more easily ionized than a "downhill" state because it can make a transition to a "downhill" state as the system traverses through zero-field avoided crossings. The model also explains the variation of the ionization threshold of the two "downhill" states with the same $k$ but with opposite orientation. Depending on the relative polarization of the static field with respect to that of the $\mathrm{THz}$ field, a state may traverse the zero-field avoided-crossings zero times, once $\left(+F_{\text {static }}\right)$ or twice $\left(-F_{\text {static }}\right)$ prior to ionization. With a positive static field applied, the "downhill" state may be ionized at the peak of the positive halfcycle of the $\mathrm{THz}$ field before experiencing the zero crossing of the field, or it may transfer into an "uphill" state and be ionized at the peak of the negative half-cycle. 
Which ionization pathway it follows depends on the ratio of the ionization threshold between the "uphill" and "downhill" states and the asymmetry between the positive and negative half-cycles of the $\mathrm{THz}$ waveform. Nevertheless, both pathways give a higher ionization threshold than that of the same "downhill" state but for a negative static field applied. In this case the electron maintains its "downhill" orientation after traversing the zero-field avoided-crossing twice and ionizes at the peak of the negative half-cycle.

However, not all of the features of the data are reproduced by the simple model. Based on the calculation, we would expect the ionization threshold to be different between the two "uphill" states for positive and negative static field applied as well, but this is not observed in the experiments. Also, the model predicts that the minimum ionization threshold for states in a Stark manifold is equal to that of the zero-field $d$ state. In the experiments, this is approximately correct for $n=10$, but not for $n=11$ or $n=12$. Our calculation assumes a hydrogenic over-the-barrier ionization from a single, isolated $n$-manifold. For Na, the zero-field avoid crossings may not be traversed purely diabatically due to the large quantum defects of the $s$ and $p$ states, and any given Stark state may project onto several Stark levels as the system traverses through zero-field avoided crossings. Numerical simulations including the multi-level Landau-Zener transition at zero-field avoided crossings have been performed, but the end results are qualitatively similar to those of the simpler model proposed here.

In conclusion, we have explored the ionization of oriented Rydberg-Stark states using intense, single-cycle THz pulses. The peak THz field strength required for ionization shows a pronounced variation among states with different permanent dipole moments but with nearly identical binding energies. A hydrogenic model qualitatively reproduces some of the major features in the data, attributing the orientation-dependent ionization to both diabatic population transfer between "uphill" and "downhill" ori- 
ented states and an asymmetry in the single-cycle $\mathrm{THz}$ waveform. Interestingly, measurements of the orientation-dependence in ionization can be used to test the asymmetry in the field and the field asymmetry can be used to control the ionization phase and electron emission direction.

\section{Prospective study: Single-cycle $\mathrm{THz}$ driven electron scattering from ori- ented atoms or molecules}

As mentioned in Chap. 1, electron rescattering that follows laser induced atomic or molecular ionization enables high harmonic generation. Because electrons are accelerated for proportionally longer periods of time in lower frequency fields, an effective way of producing electrons with higher recollision energies and obtaining higher-order harmonic generations is to use lower-frequency/longer-wavelength driving lasers. Typically IR or MIR lasers are used to drive electron rescattering. Even so, for applications such as recollision imaging, there are complications due to the uncertainty in the precise shape of the electron wavepacket and the influence of the strong field on the structure of the parent ion to be imaged. As an alternative, we have considered single-cycle $\mathrm{THz}$ driven electron scattering from oriented atoms or molecules. The basic idea is as follows: A high-lying (high principal quantum number $n$ ), oriented Rydberg-Stark state (extreme blue or red Stark state) is prepared via laser excitation in the presence of a small static electric field. The Rydberg electrons which are preferentially localized far from the ionic core on one side of the atom are then exposed to single-cycle $\mathrm{THz}$ pulses, whose field polarization axis is parallel to that of the static electric field. Initially the electron is (nearly) at rest with a well characterized spatial probability distribution. In this case, strong field ionization is not required to produce the electron projectile and the electron can be directly 
driven back towards its parent ionic core when the THz field is applied. Although the $\mathrm{THz}$ field is sufficiently strong to drive the Rydberg electron to high energies, it has negligible influence on tightly bound electrons in the ion core. The single-cycle nature of the field eliminates multiple recollisions, and the orientation of the extreme Rydberg-Stark state confines the recollision in one direction relative to the atom and enhances the recollision probability. Importantly, for a high- $n$ state, the $\mathrm{THz}$ pulse duration is much shorter than the classical Kepler period of the electron $\left(T \simeq 2 \pi n^{3}\right)$, meaning that the electron-field interaction is diabatic and the recollision wavepacket remains well characterized during propagation.
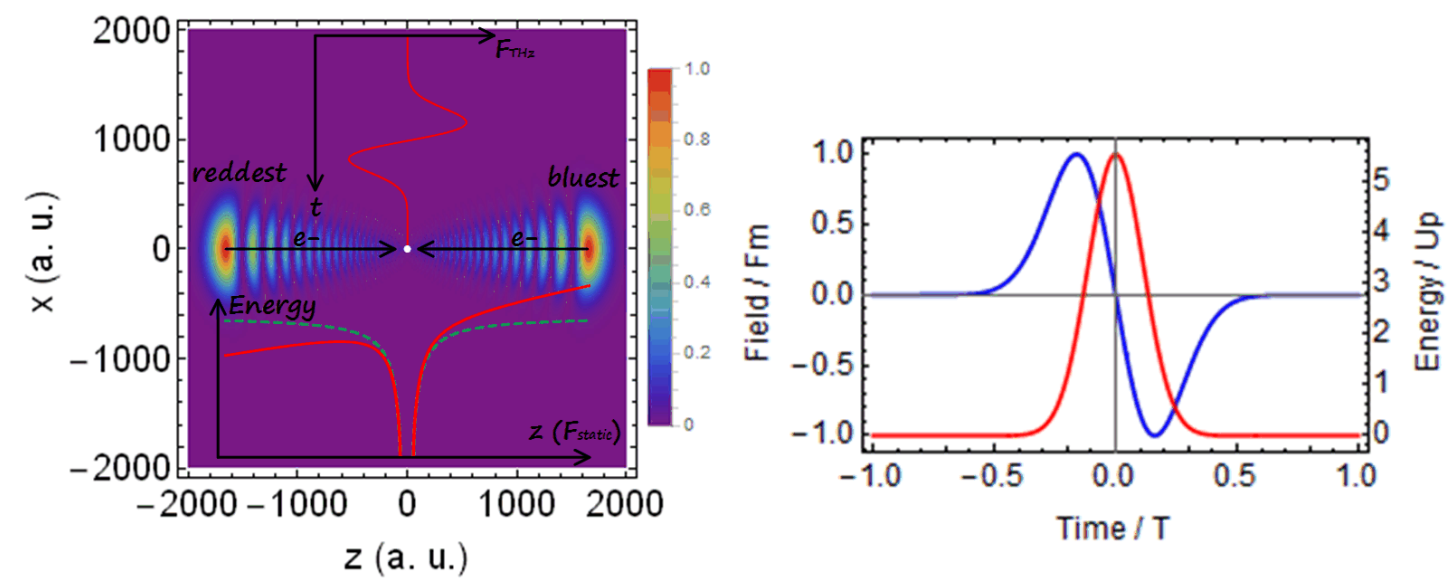

Figure 4.5: Illustration of single-cycle $\mathrm{THz}$ driven electron scattering from Rydberg-Stark eigenstates. Left) The color scale represents the electron charge density (in arbitrary units) in the $x z$ plane for a hydrogenic $n=30$ extreme blue (right) and red (left) Stark eigenstates, for a small static electric field applied in the $+z$ direction. The choice of blue or red Stark state for electron scattering depends on the direction of the $\mathrm{THz}$ field relative to that of the static field. For the configuration shown in Fig. 4.5, a blue state should be chosen. Right) Blue: Single-cycle waveform given by Eq. 4.3. Red: Recollision energy as a function of recollision time.

For a single-cycle driving pulse with Gaussian shaped vector potential, the electric field can be expressed as [131],

$$
F=-\sqrt{2} e^{1 / 2} F_{m} \frac{t}{\tau} e^{-\left(\frac{t}{\tau}\right)^{2}}
$$


For this pulse, $F_{m}$ and $\omega \simeq \frac{\pi}{2.2 \tau}$ (or $f \simeq \frac{1}{4.4 \tau}$ ) are, respectively, the maximum amplitude and peak frequency of the field. Ignoring the Coulomb potential and assuming the Rydberg-Stark electron is initially located at rest at a distance $2 n^{2}$ away from its parent ionic core, the maximum recollision energy is obtained if the electron experiences the first half-cycle of the field and recollides at $t=0$. Therefore, the principal quantum number that gives the maximum recollision energy is calculated to be,

$$
n_{\max } \simeq 94.17 \frac{\sqrt{F_{m}}}{f}
$$

and the maximum recollision energy is,

$$
E_{\text {max }} \simeq 61.74 \frac{F_{m}^{2}}{f^{2}} \mathrm{eV}
$$

Here $F_{m}$ and $f$ are the values of maximum pulse amplitude and carrier frequency in units of $\mathrm{MV} / \mathrm{cm}$ and $\mathrm{THz}$, respectively.

We have performed experiments on barium and rubidium using $f \simeq 0.2 \mathrm{THz}$ and $F_{m} \simeq 0.5 \mathrm{MV} / \mathrm{cm}$, single-cycle $\mathrm{THz}$ pulses to see if non-sequential double ionization (NSDI) from $\mathrm{THz}$ driven electron scattering can be observed $\left(\mathrm{IP}_{\mathrm{Ba}^{+}} \simeq 10 \mathrm{eV}\right.$, $\left.\mathrm{IP}_{\mathrm{Rb}^{+}} \simeq 27.3 \mathrm{eV}\right)$. The atoms were excited to a high-lying Rydberg-Stark state and then exposed to single-cycle THz pulses, unfortunately no NSDI were observed. We have also attempted to directly laser excite the atoms to just above the ionization limit immediately before $\mathrm{THz}$ exposure, but no double ionization was observed. We believe that the main challenges of performing the proposed experiments are: 1) A pure single-cycle pulse (no "wiggles" before the main pulse) is required, or the electron wavepacket is easily disturbed before experiencing the main pulse. 2) Transverse spreading of the electron wavepacket over ps time scale lowers the recollision probability. 3) Our laser repetition rate $(15 \mathrm{~Hz})$ is too low to observe small probability events. A kHz repetition rate system would be better. 4) Very high resolution laser 
is required for laser-excitation to high-lying Rydberg-Stark states ${ }^{3}$. Our self-built Littman dye laser has a resolution (FWHM linewidth) of about $0.25 \mathrm{~cm}^{-1}$. With this resolution, we can distinguish adjacent Rydberg-Stark states in the $n \simeq 30$ manifold. Using a dye-amplified diode-laser provided a resolution of about $0.01 \mathrm{~cm}^{-1}$ enabling the excitation of higher lying Stark states in Rb. Adjacent Rydberg-Stark states in the $n \simeq 70$ manifold are distinguishable with this diode laser ${ }^{4}$. Assuming a THz waveform given by Eq. 4.3 with $f \simeq 0.2 \mathrm{THz}$ and $F_{m} \simeq 0.5 \mathrm{MV} / \mathrm{cm}$, electrons in $n=30$ and $n=70$ states obtain approximately $0.35 \mathrm{eV}$ and $6.7 \mathrm{eV}$ recollision energies, respectively, insufficient to induce double ionization in either $\mathrm{Ba}$ or $\mathrm{Rb}$.

From Eq. 4.4 and Eq. 4.5, it is apparent that it would be advantageous to use higher frequency $\mathrm{THz}$ radiation with higher peak field strengths, e.g., for $f=10$ $\mathrm{THz}$ and $F_{m}=10 \mathrm{MV} / \mathrm{cm}, n_{\max } \simeq 30$ and $E_{\max } \simeq 62 \mathrm{eV}$. The development of such $\mathrm{THz}$ sources may may provide better opportunities for future Rydberg recollision experiments.

\footnotetext{
${ }^{3}$ In a $n$-manifold, for an applied static field $F_{\text {static }}=1 /\left(3 n^{5}\right)$, the energy separation between adjacent Stark levels is $\Delta E=3 F n=1 / n^{4}$ (a. u.) $=2.195 \mathrm{E} 5 / n^{4} \mathrm{~cm}^{-1}=6.58 \mathrm{E} 9 / n^{4} \mathrm{MHz}$.

${ }^{4}$ This is assuming that two states separated by FWHM linewidth of the laser are distinguishable, and ignoring the thermal Doppler broadening.
} 
Chapter 5

High-Energy Electron Emission

from Metallic Nano-tips Driven by Intense Single-Cycle $\mathrm{THz}$ Pulses 
Chapter 5. High-Energy Electron Emission from Metallic Nano-tips Driven by Intense Single-Cycle THz Pulses

\begin{abstract}
The mechanisms through which electrons are ejected from atoms (or molecules) and subsequently driven to high-energies in a strong oscillating electric field are generally well understood, enabling a variety of techniques from attosecond pulse generation to imaging via time-resolved electron scattering. Analogous processes are at play in strong-field emission and acceleration of electrons from solids and, for nano-scale targets, brief electron bursts from particles with dimensions much less than the wavelength of the drive field hold great promise for ultrafast imaging and other applications. For long wavelength drivers, the enhanced local field near nano-particles or nano-tips can fundamentally change the energy transfer process and allow for the creation of very high energy electrons. Here we report on the use of intense single-cycle $\mathrm{THz}$ pulses to drive electron emission from unbiased nano-tipped wires. Energies easily exceeding $5 \mathrm{keV}$ are observed, substantially greater than those previously attained at higher $\mathrm{THz}$ and infrared frequencies. In addition, despite large differences in the magnitude of the respective local fields, we find that the maximum electron energies are only weakly dependent on the tip radius, for $10 \mathrm{~nm}<R<1 \mu \mathrm{m}$. Due to the single-cycle nature of the field, the high-energy electron emission is predicted to be confined to a single burst, potentially enabling a variety of applications.
\end{abstract}


Chapter 5. High-Energy Electron Emission from Metallic Nano-tips Driven by Intense Single-Cycle THz Pulses

Optical-field driven electrons are a hallmark of intense laser physics and attosecond science $[79,80]$. They are the basis for attosecond pulse generation and enable a variety of techniques for probing dynamics with atomic-scale temporal and/or spatial resolution [109, 132-139]. However, for condensed matter targets, laser-induced surface and bulk damage limit the maximum visible or near infrared drive intensities that can be applied, reducing the magnitude of the electronic momentum transfer and the viability of these probe methods. To combat this issue, enhanced local fields in the vicinity of metallic nano-structures and longer wavelength drivers can be exploited to significantly reduce the optical intensities required to induce emission and accelerate electrons to high energies. Indeed, during the last decade, numerous studies have explored strong-field physics by coupling femtosecond laser pulses to nanostructured solids, particularly metallic nano-tips [140-154]. The results of those investigations demonstrated that, with proper modification, much of the basic physics underlying laser induced ionization of atomic and molecular gases also applies to laser-induced electron emission from solids. Specifically, different mechanisms, including photoemission, photo-field emission, and optical field emission, dominate in different frequency regimes, and the well-known Keldysh parameter [17] can be adapted to define the transition from photon- to field-induced processes [140] (For metals, $\gamma=\sqrt{\Phi /\left(2 U_{p}\right)}$, where $\Phi$ is the metal work-function and $U_{p}$ is the ponderomotive energy). The classical notion of field-driven electron dynamics, originally employed to understand the response of gas-phase systems to intense laser pulses, can be readily applied to nanostructured targets as well. Examples of related phenomena include ponderomotive acceleration and rescattering of ejected electrons [143, 153], carrier-envelope phase (CEP) effects [143, 153], and multiphoton/strong-field atomic excitation and ionization induced extreme-ultraviolet (XUV) fluorescence [155-157]. That said, the substantial local-field enhancements in the vicinity of nanostructured materials can 
Chapter 5. High-Energy Electron Emission from Metallic Nano-tips Driven by Intense Single-Cycle THz Pulses

lead to key differences in the details of how, and how much, energy and momentum are transferred to the driven electrons [140, 150, 152, 153]. For example, when exploring electron emission from gold nanotips as a function of drive laser wavelength, Herink et al. observed a transition from the familiar "quiver" response of electrons in an oscillating field, to a new "sub-cycle" energy transfer regime [140]. At sufficiently long wavelengths, ejected electrons leave the vicinity of the nanotip (and the enhanced field) in a fraction of the optical-cycle, resulting in a maximum energy transfer that is proportional to the drive field at the instant of emission.

At still longer wavelengths, field emission at microwave frequencies was observed in the 1960s $[158,159]$. Also, $\mathrm{THz}$ radiation has recently been employed to control photoemission from nano-tips [160] and as a temporal gate to enable time-resolved scanning tunneling microscopy [161]. However, due primarily to the lack of sufficiently intense table-top $\mathrm{THz}$ sources, electron field-emission in the presence of $\mathrm{THz}$ radiation alone has only very recently been reported $[162,163]$. Further investigation of the THz-induced emission and subsequent energy transfer is motivated by both fundamental science and applications. For example, because THz photon energies are three orders of magnitude smaller than the work functions of typical metals, ultrafast emission at $\mathrm{THz}$ frequencies enables access to purely field-driven, rather than photon-mediated processes. In metals with sufficiently low scattering, coherent energy transfer to electrons prior to ejection could result in highly non-equilibrium conditions which, among other things, might lead to substantial deviations of the emission process from purely adiabatic tunneling [162]. Moreover, at $\mathrm{THz}$ frequencies, the local electron-field interaction can be pushed far deeper into the sub-cycle regime as compared to that at mid-infrared wavelengths [140]. Ejected electrons can be driven to very high energies in the enhanced local field of metallic nano-tips, potentially enabling time-resolved streaking or providing spatially and temporally localized electron 
Chapter 5. High-Energy Electron Emission from Metallic Nano-tips Driven by Intense Single-Cycle THz Pulses

bursts of high energy electrons for probing matter [163, 164]. Also, in contrast to the optical or infrared regimes, true single-cycle drive fields can be utilized, confining the high-energy electron emission to a single, isolated burst. Finally, the enhanced $\mathrm{THz}$ field in the vicinity of metallic tips could serve as a quasi-static field capable of influencing intense laser-induced electron rescattering processes in a surrounding atomic or molecular gas, potentially increasing the high-energy cutoff or photon yield in HHG [165-167] or eliminating multiple electron returns that complicate the interpretation of recollision imaging measurements [137-139].

Given this motivation, we have investigated electron emission from tungsten wire tips that have been exposed to intense, single-cycle, $\mathrm{THz}$ pulses. We observe electron energies in excess of $5 \mathrm{keV}$, more than an order of magnitude greater than those previously reported in the near-infrared, infrared, and $\mathrm{THz}$ regimes, implying the presence of acceleration gradients as large as $250 \mathrm{GeV} / \mathrm{m}$. It is likely that we have produced electrons with energies in excess of $10 \mathrm{keV}$ using the highest $\mathrm{THz}$ fields available in our laboratory, but these have yet to be confirmed due to voltage limitations in our current spectrometer. The maximum electron energies are directly proportional to the peak $\mathrm{THz}$ field, reflecting large local-field enhancement factors that are inversely proportional to the tip radius, $R$, and greater than 3000 for the sharpest tips. We have measured the emission from tips whose radii (and local field enhancement factors) differ by nearly two orders of magnitude and, interestingly, find that the maximum electron energy varies by only a factor of approximately two at any incident THz field strength. This result can be understood by modeling the sub-cycle energy transfer in the locally enhanced field of the wire tip.

In the experiments, electrochemically-etched tungsten wire tips (e.g. see Fig. 5.3a for typical SEM images) are exposed, in vacuum, to intense single-cycle THz pulses. As illustrated in Fig. 5.1a, electrons emitted from an electrically grounded tip, which 
Chapter 5. High-Energy Electron Emission from Metallic Nano-tips Driven by Intense Single-Cycle THz Pulses

is mounted in a static-field-free region of a retarding potential spectrometer, are collected by a micro-channel plate (MCP) detector. The electron energy spectrum is measured by varying the magnitude of a retarding potential placed on the entrance to the MCP. For direct comparison of the emission characteristics of different tips under identical conditions, several tips with different radii and/or cone angle can be simultaneously mounted in the spectrometer. The $6 \mathrm{~mm}$ separation between adjacent wires is large compared to the $\sim 2 \mathrm{~mm} \mathrm{THz}$ focus, ensuring emission from one, and only one, tip in any given measurement. A precision translation stage is used to move individual tips into and out of the $\mathrm{THz}$ focus, enabling measurements with different tips under identical experimental conditions. In most cases, the tungsten wires are mounted parallel to the spectrometer axis with their tips pointed directly toward the $\mathrm{MCP}$, and the positive half-cycle of the $\mathrm{THz}$ field is directed from the wire tip to the MCP. In this standard configuration, electron emission cannot occur during the positive half-cycle of the single-cycle $\mathrm{THz}$ field (see Fig. 5.1b). Instead, the negative half-cycle is responsible for the field emission and subsequent energy transfer.

The $\mathrm{THz}$ pulses used in the experiments are characterized via photoelectron streaking in the same vacuum apparatus and spectrometer used for the field emission experiments [124]. The pulses are passively CE-phase stable, with single-cycle sine-like waveforms, a central frequency $f \simeq 0.15 \mathrm{THz}$, a maximum field strength (at focus) of approximately $450 \mathrm{kV} / \mathrm{cm}$, and a field amplitude asymmetry of $\sim 2 / 3$ between the positive and negative half cycles. Fig. 5.1b shows the $\mathrm{THz}$ waveform and its spectrum.

We have observed electron emission from a variety of tungsten tips having different cone angles and tip radii, $10 \mathrm{~nm}<R<1 \mu \mathrm{m}$ (see for example, Fig. 5.3a). The majority of the tips are relatively blunt in comparison with the sharper (1 to 20 $\mathrm{nm})$ tips that are typically used in studies of ultra-fast, nano-tip field emission [140- 
Chapter 5. High-Energy Electron Emission from Metallic Nano-tips Driven by Intense Single-Cycle THz Pulses

143, 146, 148-150, 157, 160-164]. Even so, as shown in Figs. 5.2 and 5.3, electron emission is observed for incident $\mathrm{THz}$ fields as low as $40 \mathrm{kV} / \mathrm{cm}$. In all cases, we find that the most probable and maximum electron energies scale linearly with the peak THz field.
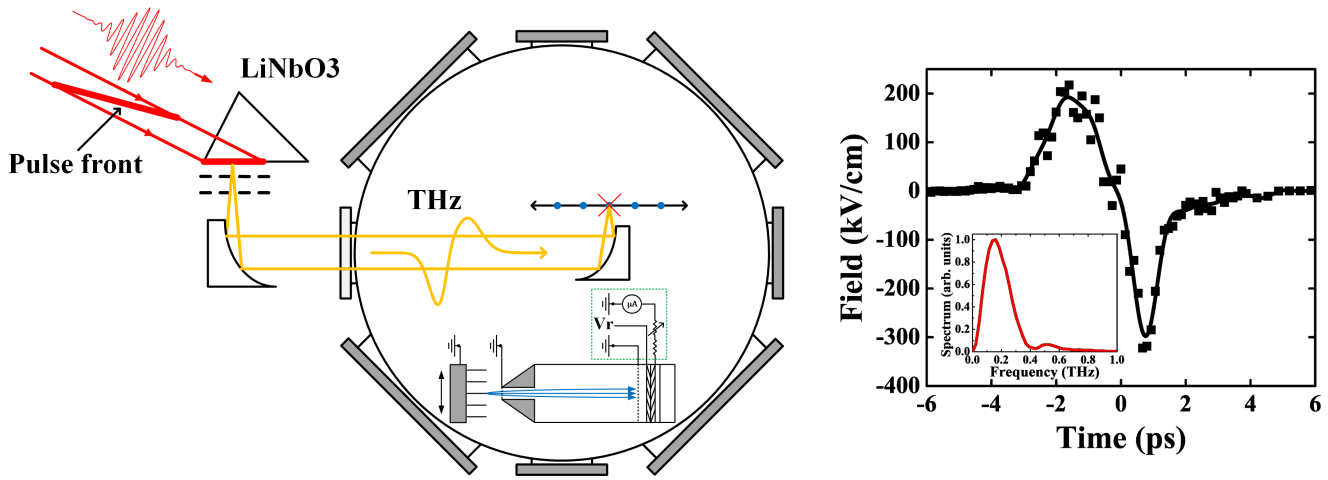

Figure 5.1: a) Schematic of the experimental apparatus. THz pulses are produced via optical rectification, at a $15 \mathrm{~Hz}$ repetition rate, using $790 \mathrm{~nm}, 150 \mathrm{fs}, 20 \mathrm{~mJ}$ pump laser pulses in a tilted pulse-front configuration. The THz pulses pass through two wire-grid polarizers which serve as a variable attenuator. Two off-axis parabolic mirrors focus the THz radiation on a single wire tip positioned at the center of a retarding-potential electron energy analyzer. The tips are mounted in a grounded conducting holder that is rigidly attached to a mechanical translation stage, which allows the positioning of different tips in the $\mathrm{THz}$ focus, allowing us to maintain identical experimental conditions for measurements from different tips without breaking vacuum. The holder can accommodate up to 5 wires, mounted in a line with $\sim 6 \mathrm{~mm}$ gaps between adjacent tips. The gap between wires is much larger than the $\sim 2 \mathrm{~mm}$ focal diameter of the $\mathrm{THz}$ beam, ensuring that only a single tip is exposed to the field at any given time. b) THz electric field (main figure) obtained from photoelectron streaking measurements [124] and corresponding frequency spectrum (inset) obtained by Fourier transforming the time-domain data shown in the main figure.

The data in Figs. 5.2a,b,d,e and 5.3 were obtained in a "parallel" geometry in which the $\mathrm{THz}$ polarization, the tungsten wire, and the spectrometer axis are colinear. No electrons are detected when the wire is oriented perpendicular to the spectrometer axis, indicating that the emission is confined to an acute solid angle centered along the wire axis. In addition, comparison of the electron yield as a function of 
Chapter 5. High-Energy Electron Emission from Metallic Nano-tips Driven by Intense Single-Cycle THz Pulses

$\mathrm{THz}$ polarization direction (at fixed $\mathrm{THz}$ field strength), with the yield obtained as a function of $\mathrm{THz}$ field strength in the parallel wire-field geometry, confirms that the emission depends only on the component of the applied field parallel to the wire axis (See Fig. 5.2c). Therefore, in the remainder of this study, we focus on results obtained in the parallel wire-field configuration.
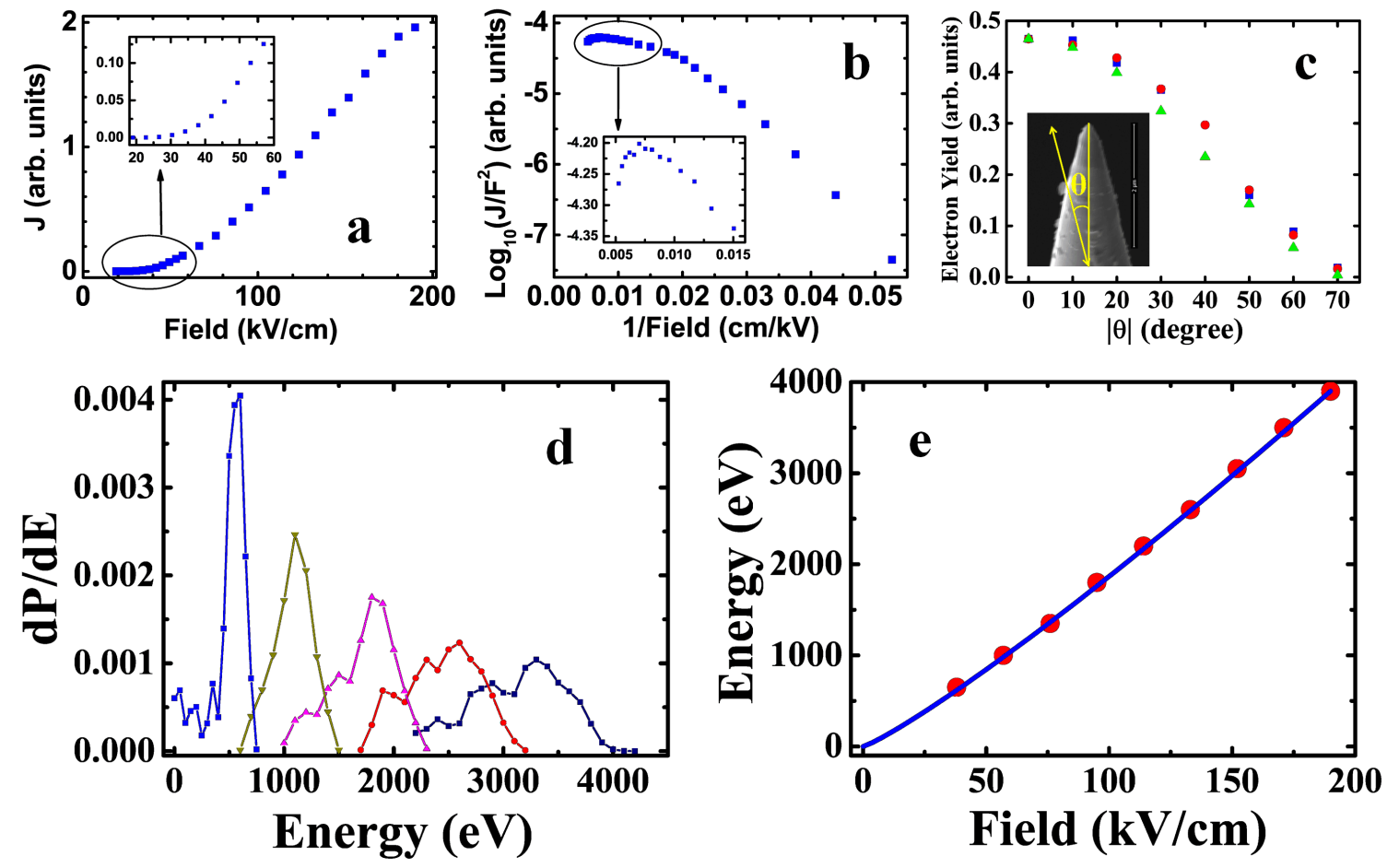

Figure 5.2: Results of $\mathrm{THz}$ induced field emission from a tip of radius $R \simeq 130 \mathbf{n m}$. a) Electron emission current as a function of incident $\mathrm{THz}$ field strength. b) Dependence of electron current on incident $\mathrm{THz}$ field strength plotted using standard Fowler-Nordheim scaled parameters. c) Electron current at fixed incident $\mathrm{THz}$ field strength, as a function of field polarization angle relative to the tip axis. positive $\theta$ (blue); negative $\theta$ (red); calculation (green). The calculation uses the measurements shown in b) and assumes that the effective field is the field component along the tip axis. Inset: SEM image of the tip. d) Energy distribution of emitted electrons for different incident THz fields: $40 \mathrm{kV} / \mathrm{cm}$ (light blue); $80 \mathrm{kV} / \mathrm{cm}$ (green); $110 \mathrm{kV} / \mathrm{cm}$ (magenta); $150 \mathrm{kV} / \mathrm{cm}$ (red); $190 \mathrm{kV} / \mathrm{cm}$ (dark blue). Lines connecting data points are provided to guide the eye. e) High-energy cutoff of electron energy spectra as a function of incident $\mathrm{THz}$ field strength. Solid red circles are data and the solid blue line is the result of the simulation described in the text. 
Chapter 5. High-Energy Electron Emission from Metallic Nano-tips Driven by Intense Single-Cycle THz Pulses

In general, at low $\mathrm{THz}$ fields the electron current vs. field exhibits the expected exponential form for Fowler-Nordheim tunneling [28]. However, at higher fields the yields saturate and exhibit a more complex field-dependent behavior (see Figs. 5.2a and $5.2 \mathrm{~b}$ ), perhaps due in part to non-adiabatic energy transfer to the electrons within the wire. That said, our principal interest in this study is not on the mechanism responsible for liberating electrons from the tip, but rather on the process through which those electrons obtain such large kinetic energies from the field.

We first consider how the energies of the emitted electrons from a single tip depend on the applied $\mathrm{THz}$ field. As a typical example, Fig. 5.2d shows the energy distributions of the electrons emitted from an $R \sim 130 \mathrm{~nm}$ tip at five different $\mathrm{THz}$ fields. The maximum energies observed are plotted as a function of $\mathrm{THz}$ field strength in Fig. 5.2e. The general shape of each distribution, a well-defined peak with a sharp high-energy cutoff, are similar to those previously reported for $\mathrm{THz}$ induced field emission [162], and the linear scaling of the cutoff energy vs incident field is a signature of energy transfer in the strong-field, sub-cycle regime [140]. The features in Fig. $5.2 \mathrm{~d}$ have low energy tails that are less pronounced than those reported by Herink et al. [162], but the scaled widths (FWHM divided by peak energy), which are roughly independent of $\mathrm{THz}$ field, are comparable to theirs. More interestingly, however, the distributions shown in Fig. 5.2d have peak energies that are approximately 30 times larger than those measured previously at nearly identical incident $\mathrm{THz}$ field strength [162]. Apparently, the substantially larger electron energies can be attributed to the lower $\mathrm{THz}$ frequency used in the current experiment $(\simeq 0.15 \mathrm{THz}$ vs $\simeq 1.1 \mathrm{THz})$, and/or the larger tip radius ( $\simeq 130 \mathrm{~nm}$ vs $\simeq 10 \mathrm{~nm}$ ). The maximum energies we observe are also more than an order of magnitude larger than those produced with mid-infrared pumping at intensities that are 5 orders of magnitude higher [140] . 
Chapter 5. High-Energy Electron Emission from Metallic Nano-tips Driven by Intense Single-Cycle THz Pulses
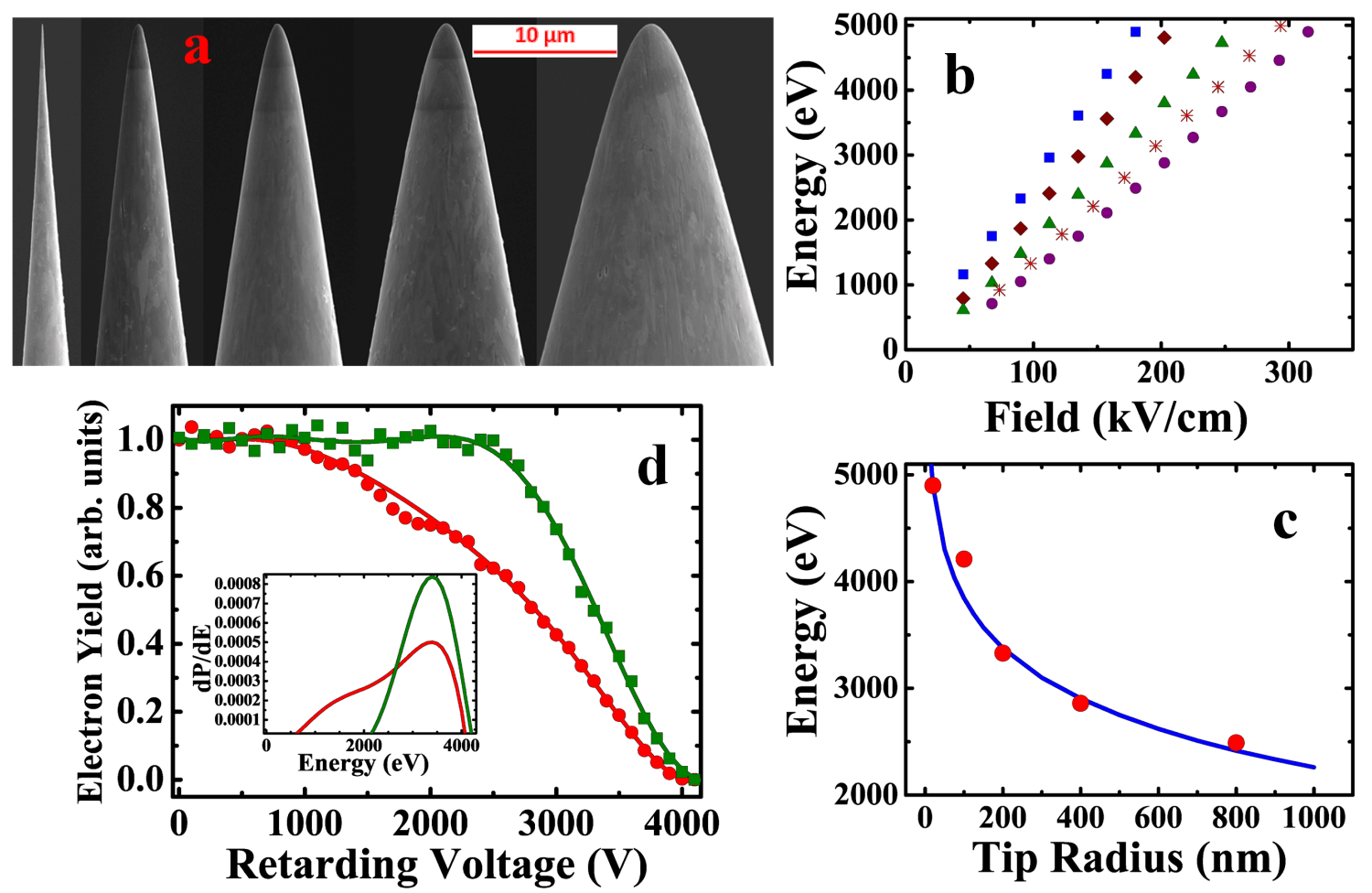

Field (kV/cm)

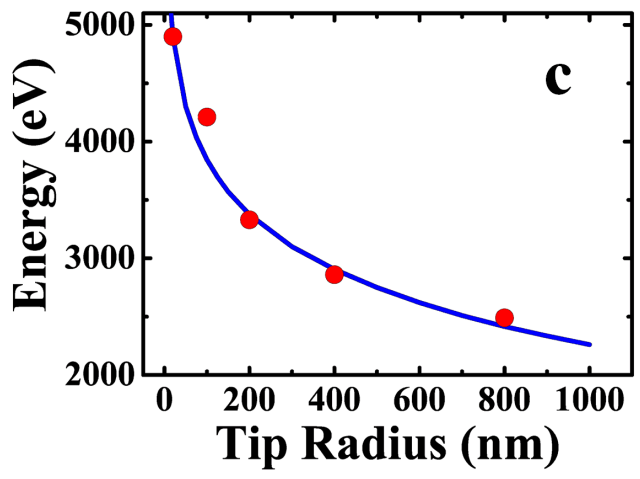

Figure 5.3: Comparison of $\mathrm{THz}$ induced field emission from different tips. a) SEM images of the five tungsten tips used to produce the data shown in b-d. From left to right, the tip radii are: $R \simeq 20,100,200,400$, and $800 \mathrm{~nm}$. b) High-energy cutoff of the electron energy spectrum as a function of incident $\mathrm{THz}$ field strength, for tips with $R \simeq 20 \mathrm{~nm}$ (filled blue squares), $100 \mathrm{~nm}$ (filled maroon diamonds), $200 \mathrm{~nm}$ (filled green triangles), $400 \mathrm{~nm}$ (red asterisks), and $800 \mathrm{~nm}$ (filled purple circles). c) Electron cutoff energy as a function of tip radius for a fixed incident $\mathrm{THz}$ field strength $F \simeq 180 \mathrm{kV} / \mathrm{cm}$. d) Main Figure: Electron yield vs. retarding voltage (data points) and smooth fits to those data (solid lines) for tips with radii of $R \simeq 100 \mathrm{~nm}$ (green) and $R \simeq 800 \mathrm{~nm}$ (red). Inset: Energy distributions for tips with radii of $R \simeq 100 \mathrm{~nm}$ (green) and $R \simeq 800 \mathrm{~nm}$ (red) obtained from the smooth fits to the yields shown in the main figures. The data were collected using different $\mathrm{THz}$ field strengths $(\sim 180$ $\mathrm{kV} / \mathrm{cm}$ and $\sim 270 \mathrm{kV} / \mathrm{cm}$ for the $100 \mathrm{~nm}$ and $800 \mathrm{~nm}$ tips, respectively) to generate spectra with similar cutoff energies, $\sim 4 \mathrm{keV}$.

To better understand the mechanism responsible for the high electron energies produced in our experiments, we have also examined the electron emission as a function of tip radius and cone angle. Fig. 5.3a shows SEM images of 5 tips with radii $R \simeq$ 20, 100, 200, 400, and $800 \mathrm{~nm}$. Fig. 5.3b shows the high-energy cutoff of the electron 
Chapter 5. High-Energy Electron Emission from Metallic Nano-tips Driven by Intense Single-Cycle THz Pulses

distributions from each tip as a function of incident $\mathrm{THz}$ field. For sufficiently high incident fields, electrons with energies exceeding $5 \mathrm{keV}$ are produced from all of the tips. However, due to limitations of the retarding potential spectrometer, only cutoff energies $<5 \mathrm{keV}$ can be measured. Fig. $5.3 \mathrm{c}$ shows the variation in cutoff energy with tip radius for a fixed incident $\mathrm{THz}$ field of $180 \mathrm{kV} / \mathrm{cm}$. Naively, one might expect that the highest energy electrons would be produced from the sharpest tips, due to the larger local field enhancement. This is indeed the case, but perhaps not to the degree anticipated. Notably, we observe no significant difference in the cutoff energies from tips with approximately the same tip radius but with cone angles differing by as much as $60 \%$. Moreover, for a given incident field, we observe only a factor of two decrease in the maximum electron energy when the tip radius is increased by a factor of 40 (Figs. 5.3b and 5.3c).

The linear, rather than quadratic, scaling of the maximum electron energy with incident field strength indicates that the underlying physical mechanism for the energy transfer is substantially different from that predicted by the simpleman's model for electron emission in the presence of an oscillating field. Indeed, our observations are consistent with a different model [140] in which the energy transfer to the electron is confined to a fraction of the field cycle. As such, it depends on the magnitude of the locally enhanced field at the instant of emission, rather than on the vector potential (which reflects the time-integral of the field after emission).

The sub-cycle energy transfer process as it applies to our measurements can be understood as follows. When a tip with radius of curvature, $R$, is exposed to a $\mathrm{THz}$ field, the induced charge at the surface of the tip modifies the local field surrounding it. A discrete, driven, series-RC circuit model indicates that for tungsten tips with radii $R>50 \mathrm{~nm}$ driven at wavelengths comparable to, or greater than that used in our experiments, the induced surface charge (and field) at the tip oscillate approximately 
Chapter 5. High-Energy Electron Emission from Metallic Nano-tips Driven by Intense Single-Cycle THz Pulses

in-phase with the incident field. As a result, the field enhancement factor $\gamma$ can be computed in the static field limit. In contrast, at higher frequencies and for smaller radius tips the decreased capacitive reactance and/or increased resistance can result in a non-negligible phase shift between the incident and enhanced fields, limiting the local enhancement. For our measurements, the surface field at any instant can be written as, $F=\gamma F_{0}$, where $F_{0}$ is the incident $\mathrm{THz}$ field. An electron, ejected from the surface, rapidly accelerates away from the tip in the locally enhanced field. The local field drops rapidly to $F_{0}$ as the electron travels a distance comparable, and proportional, to $R$ [168]. The electron leaves the vicinity of the tip in a time much less than the $\mathrm{THz}$ period, so the incident field remains roughly constant during that time. The electron's energy gain is equal to the work done by the enhanced local field which, in turn, is approximately equal to the average electric force, $\frac{1}{2} \gamma F_{0}$, multiplied by the distance, $R$, over which the enhanced field decays,

$$
\Delta E=\frac{1}{2} \gamma F_{0} R \text { (a.u.). }
$$

For $\gamma \gg 1$, this local energy transfer is much greater than that acquired by the electron (up to $8 U_{p}$ ) as it moves in the bare, single-cycle $\mathrm{THz}$ field far from the tip [124]. According to Eq. 5.1, the final energy, $\Delta E$, of an ejected electron will be proportional to the magnitude of the incident field at the instant of its birth, with the maximum electron energy proportional to the peak $\mathrm{THz}$ field. This prediction is in excellent agreement with our measurements (see Figs. 5.2e and 5.3b).

The sub-cycle energy transfer model also explains the (near) independence of $\Delta E$ on $R$. To see this, $\gamma$ must be expressed in terms of relevant geometric parameters. For a hemisphere-capped cylinder of length $L$ and radius $R$ in a static field, $\gamma$ is approximately proportional to $\sim L / R$ [35]. However, in the presence of $\mathrm{THz}$ radiation of wavelength $\lambda$, retardation effects will limit the effective length of the emitter to 
Chapter 5. High-Energy Electron Emission from Metallic Nano-tips Driven by Intense Single-Cycle THz Pulses

$L<\lambda / 2[169]$. As a result, Eq. 5.1 reduces to,

$$
\left.\Delta E \sim \frac{1}{4} F_{0} \lambda \quad \text { (a.u. }\right)
$$

Beyond the linear dependence on the applied field, Eq. 5.2 predicts an energy transfer that is directly proportional to the $\mathrm{THz}$ wavelength, and independent of tip radius. Electrons ejected from sharper tips experience a larger enhanced field as compared to blunter tips, but the field decays over a proportionally shorter distance. For a peak THz field strength $F_{0}=180 \mathrm{kV} / \mathrm{cm}$ and a THz wavelength $\lambda=1 \mathrm{~mm}$, Eq. 5.2 obtains a maximum energy transfer $\Delta E \sim 4.5 \mathrm{keV}$, in reasonable agreement with the measurements, $2500 \mathrm{eV} \leq \Delta E \leq 5000 \mathrm{eV}$, for $800 \mathrm{~nm} \geq R \geq 20 \mathrm{~nm}$. Although the measured energy transfer does show some $R$-dependence, that dependence is small, with a variation in $\Delta E$ of only a factor of 2 for tip radii differing by a factor of 40 .

To reproduce the (small) observed $R$-dependence in $\Delta E$, we augment the zerothorder model presented above. Specifically, approximate analytic forms for both the time-dependent tunneling probability and the spatial distribution of the enhanced field are used to compute the energy distributions of the detected electrons for 1dimensional motion along the tip axis, $z$. Rather than attempting to precisely model the geometry of the tips and compute the enhanced field via finite-difference techniques, we employ a generalized form for the field enhancement factor at the tip apex [35], $\gamma=\beta(\lambda / R)^{\alpha}$, where $\beta$ and $\alpha$ are constants to be determined. The FowlerNordheim tunneling formula [28] is used to compute the rate at which electrons are emitted (with zero initial energy) into the enhanced field. Non-relativistic, classical equations of motion are then used to determine the final energy of electrons emitted at each time, $t$, using the known waveform of the incident field and the expression, $F(z, t)=F(0, t) /(1+2 z / R)[34]$, for the decay of the electric near-field along the axis of a hyperboloidal tip. The constants $\alpha$ and $\beta$ that appear in the field enhancement 
Chapter 5. High-Energy Electron Emission from Metallic Nano-tips Driven by Intense Single-Cycle THz Pulses

factor are varied to obtain the best agreement with experiment (for more details, see Appendix J).

As shown in Figs. 5.2e and 5.3c, the simulation accurately reproduces the maximum electron energies observed as a function of peak $\mathrm{THz}$ field and tip radius for $\beta=0.06$ and $\alpha=1$. The calculations indicate that the (slight) decrease in $\Delta E$ with increasing radius is due to the greater time that the electron spends in the enhanced field, reducing the accuracy of the instantaneous field approximation used to obtain Eqs. 5.1 and 5.2. The assignment $\alpha=1$ agrees with our initial assumption that $\gamma$ is inversely proportional to $R$, while $\beta=0.06$ indicates that the effective tip length is considerably smaller than $\lambda / 2$, consistent with previous calculations for incident $800 \mathrm{~nm}$ fields [170]. The calculated maximum energies are not changed if the FowlerNordheim tunneling rate is modified to simulate the yield saturation that is observed at the highest $\mathrm{THz}$ fields in the experiments.

One feature of the data that is not satisfactorily reproduced by the simulations is the broadening of the electron energy distributions with increasing $R$ (see Appendix $\mathrm{J}$ for the simulation of electron energy spectra). Fig. 5.3d shows the energy distributions of the electrons emitted from tips with $R \simeq 100 \mathrm{~nm}$ and $R \simeq 800 \mathrm{~nm}$ at peak $\mathrm{THz}$ fields of $180 \mathrm{kV} / \mathrm{cm}$ and $270 \mathrm{kV} / \mathrm{cm}$, respectively. The distributions have approximately the same high energy cutoffs, but substantially different widths. Contrary to what is observed, based on the model presented above, we would expect a broader distribution from the sharper tip for the following reason. The sharper tip has a field enhancement factor that is approximately eight times larger than that of the blunt tip. Therefore, the sharp tip should exhibit a substantial tunneling emission rate over a much wider range of incident fields, resulting in broader distribution of final electron energies. Notably, the electrons are emitted over a much larger area with the blunt tip, and the total yield is several times lower than that from the sharper tip 
Chapter 5. High-Energy Electron Emission from Metallic Nano-tips Driven by Intense Single-Cycle THz Pulses

(as predicted given the lower field enhancement factor). Consequently, the density of electrons leaving the blunt tip is more than two orders of magnitude smaller than that from the sharp one, arguing against space-charge interactions as the source of the broadening.

Notably, due to extremely low photon energies $(\sim \mathrm{meV})$, THz radiation is nonionizing and does not cause ablation or other sample damage, potentially enabling non-destructive analysis applications. In our experiments, no damage was found for all the field emission nano-tips after tens of hours of intense $\mathrm{THz}$ exposure.

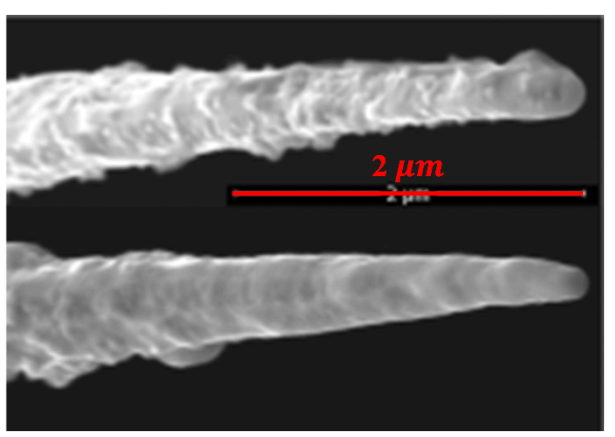

Figure 5.4: SEM images of a W nanotip before (top) and after (bottom) tens of hours of intense $\mathrm{THz}(\sim 0.5 \mathrm{MV} / \mathrm{cm})$ exposure in vacuum. No damage to the tip is found. Interestingly, the surface of the tip is smoothed with all the small features (due to imperfect etching) wiped off. Although the focal size $(\sim 2$ $\mathrm{mm}$ ) of the $\mathrm{THz}$ beam is much larger than the size of the tip, only the very end of the tip, about $2 \mu \mathrm{m}$ in length, is affected, indicating that intense field emission only occurs at the very sharp portion, where there is a huge field enhancement.

In summary, we have used strong single-cycle $\mathrm{THz}$ fields to induce electron emission from tungsten nano-tips. Electrons with energies easily exceeding $5 \mathrm{keV}$ have been observed, substantially greater than previously seen using $\mathrm{THz}$, infrared, or near infrared driving. The maximum electron energies are proportional to the peak $\mathrm{THz}$ field and roughly independent of tip radius, in good agreement with the predictions of a sub-cycle energy transfer model. Accordingly, the longer wavelength used in this work, as compared to previous photoemission studies, appears to be the primary 
Chapter 5. High-Energy Electron Emission from Metallic Nano-tips Driven by Intense Single-Cycle THz Pulses

factor responsible for the significantly higher electron energies. Following a full characterization of their temporal and angular distributions, these isolated high-energy electron bursts might serve as sources $[171,172]$ to enable a variety of applications, including time-resolved electron diffraction [164]. In addition, in this long wavelength regime, the enhanced $\mathrm{THz}$ fields in the vicinity of tips with radii, $R>1 \mu \mathrm{m}$, while insufficient to induce electron emission, could be employed to explore the influence of large quasi-static fields on HHG, attosecond pulse generation, and other phenomena based on electron rescattering in an intense laser focused near the tip [165-167].

\section{Preliminary experimental results of THz-induced field electron emission from other materials/structures}

We have examined $\mathrm{THz}$ induced field electron emission from $\mathrm{W}$ nano-tipped wires and demonstrated the dramatic differences in the energy transfer process compared to that which occurs following ionization of gas phase atoms or molecules. It would be interesting to extend these studies to other materials, for example, plasmonic metals such as $\mathrm{Au}$, and to other structures, for example, an array of nano-emitters. Here we present some preliminary results of experiments performed with $\mathrm{Au}$ nano-structure coated wires and Pt STM-cut wires.

In the early stages of the field emission project, we attempted to electrochemically grow single-crystal Au nano-wires off of the tips of silver wires that were cut and pulled to have a sharp edge. Unfortunately, we were not able to achieve single-wire growth. Instead we obtained "cluster" growths with randomly sized, distributed and aligned Au nano-structures. Fig. 5.8a shows SEM images of a silver wire with such growth. For convenience, we will call such wires Au multi-nanotipped wires in the following discussion. Exposing such wires to $\mathrm{THz}$ radiation also results in $\mathrm{keV}$ high- 
Chapter 5. High-Energy Electron Emission from Metallic Nano-tips Driven by Intense Single-Cycle THz Pulses

energy electron emissions, whereas no emission was found for a silver wire without $\mathrm{Au}$ growth. Fig. 5.5 shows the experimental data. The electron energy spectra exhibit a nearly constant amplitude plateau followed by a sharp cutoff. These spectra are quite distinct from those obtained from field emission from a single W nano-tip. In addition, the cutoff energy shows a (near) quadratic dependence on the incident $\mathrm{THz}$ field strength, dramatically different from the linear dependence for $\mathrm{W}$ nano-tips.
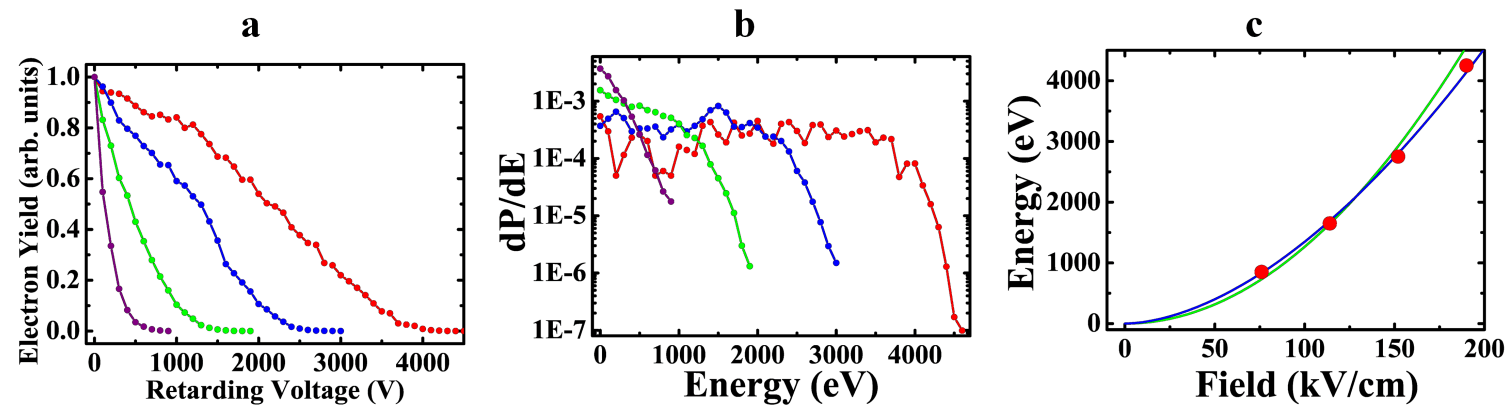

Figure 5.5: THz-induced field emission from $\mathrm{Au}$ multi-nanotipped wire: Experimental data. a) Electron Yield (maximum scaled to 1) as a function of retarding voltage at various $\mathrm{THz}$ field strengths. Red, blue, green and purple curves correspond to maximum applied THz field strengths of $F_{m}=(1.0,0.8,0.6,0.4) \times 190$ $\mathrm{kV} / \mathrm{cm}$, respectively. b) Electron energy spectra obtained from the data in a). c) Red circles: Experimental cutoff energy as a function applied $\mathrm{THz}$ field strength. Blue curve: Fit $E=0.43 F^{1.75}$ (corresponds to best linear fit of $\log E$ as a function of $\log F$ with $E$ and $F$ in units of $\mathrm{eV}$ and $\mathrm{kV} / \mathrm{cm}$, respectively). Green curve: Fit $E=0.13 F^{2}$.
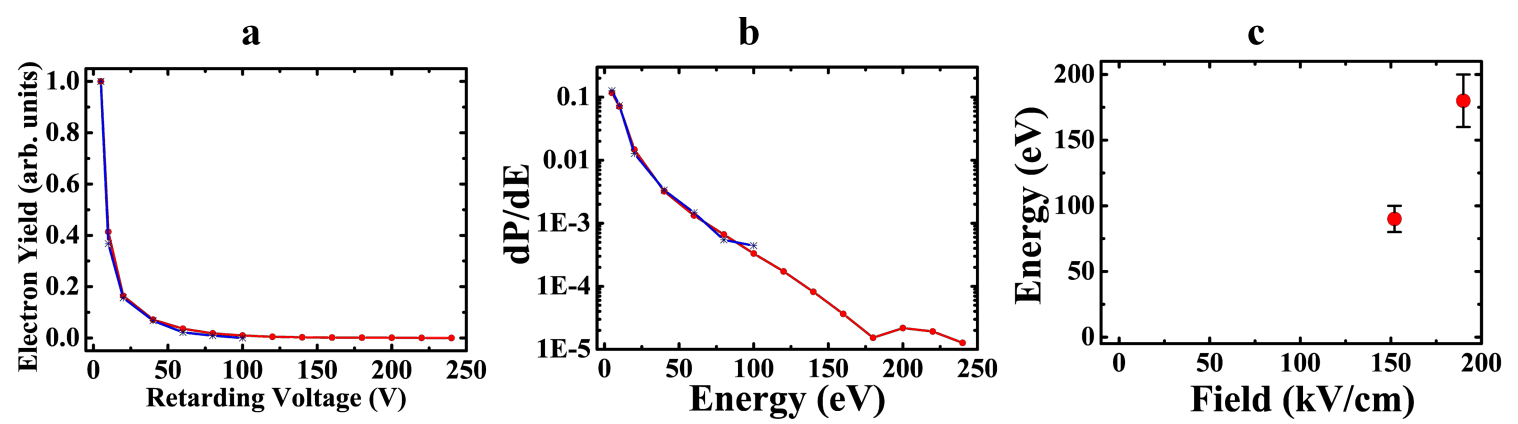

Figure 5.6: THz-induced field emission from Pt STM-cut wire: Experimental data. a) Electron yield (maximum scaled to 1) as a function of retarding voltage at various $\mathrm{THz}$ field strengths. Red and blue curves correspond to maximum applied THz field strengths of $F_{m}=(1.0,0.8) \times 190 \mathrm{kV} / \mathrm{cm}$, respectively. b) Electron energy spectra obtained from the data in a). c) Experimental cutoff energy as a function applied $\mathrm{THz}$ field strength. 
Chapter 5. High-Energy Electron Emission from Metallic Nano-tips Driven by Intense Single-Cycle THz Pulses

Since the Au multi-nanotipped wires are not well characterized in shape, it is hard to model or perform simulations. We suspect that the plateau structure of the energy spectra might be due to the coexistence of tip emitters with various sizes. We are not sure about the mechanism leading to the quadratic dependence of the electron energy on the applied field. The $\mathrm{keV}$ electron emission indicates that large field enhancements play a role. Thus we expect the energy transfer dynamics to still fall in the sub-cycle regime where the cutoff energy of electron emitted from individual emitters should depend linearly on the applied field.

We have also performed experiments with Pt wires. Such wires (commercially sold) are supposedly cut and pulled to produce a sharp tip at the end of the sharp edge. They are intended for use in scanning-tunneling-microscopy (STM) (see Fig. $5.8 \mathrm{~b}$ for SEM images). From the images, ut is clear that the Pt STM-cut wire is not very sharp, but it still has nano-scale structures and electron emission is observed when the tip is exposed to $\mathrm{THz}$ radiation. Fig. 5.6 shows the experimental results. As shown, most ( $\sim 90 \%$ ) of the emitted electrons have low energies less than $20 \mathrm{eV}$. A higher resolution energy analyzer would be needed to accurately measure the energy spectra of these slow electrons.

Fig. 5.7 uses a standard Fowler-Nordheim plot to show the dependence of fieldemitted electron current on the applied $\mathrm{THz}$ field strength for a $\mathrm{W}$ nanotipped wire, a Au multi-nanotipped wire, and a Pt STM-cut wire. In the experiments, no saturation occurs for Au multi-nanotipped wires or Pt STM-cut wires and the current-field dependence agrees with the Fowler-Nordheim prediction. However, for the $\mathrm{W}$ nanotips, current suppression at large $\mathrm{THz}$ fields is observed for both sharp and blunt tips. The high-field saturation of the emission current for $\mathrm{W}$ nanotips may be the result of charge depletion at the tip. 
Chapter 5. High-Energy Electron Emission from Metallic Nano-tips Driven by Intense Single-Cycle THz Pulses

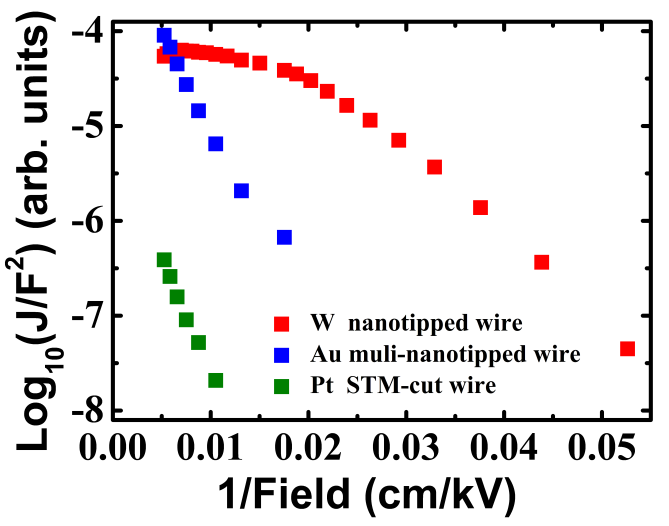

Figure 5.7: Dependence of the electron current $J$ on incident $\mathrm{THz}$ field strength $F$ plotted using standard Fowler-Nordheim scaled parameters.

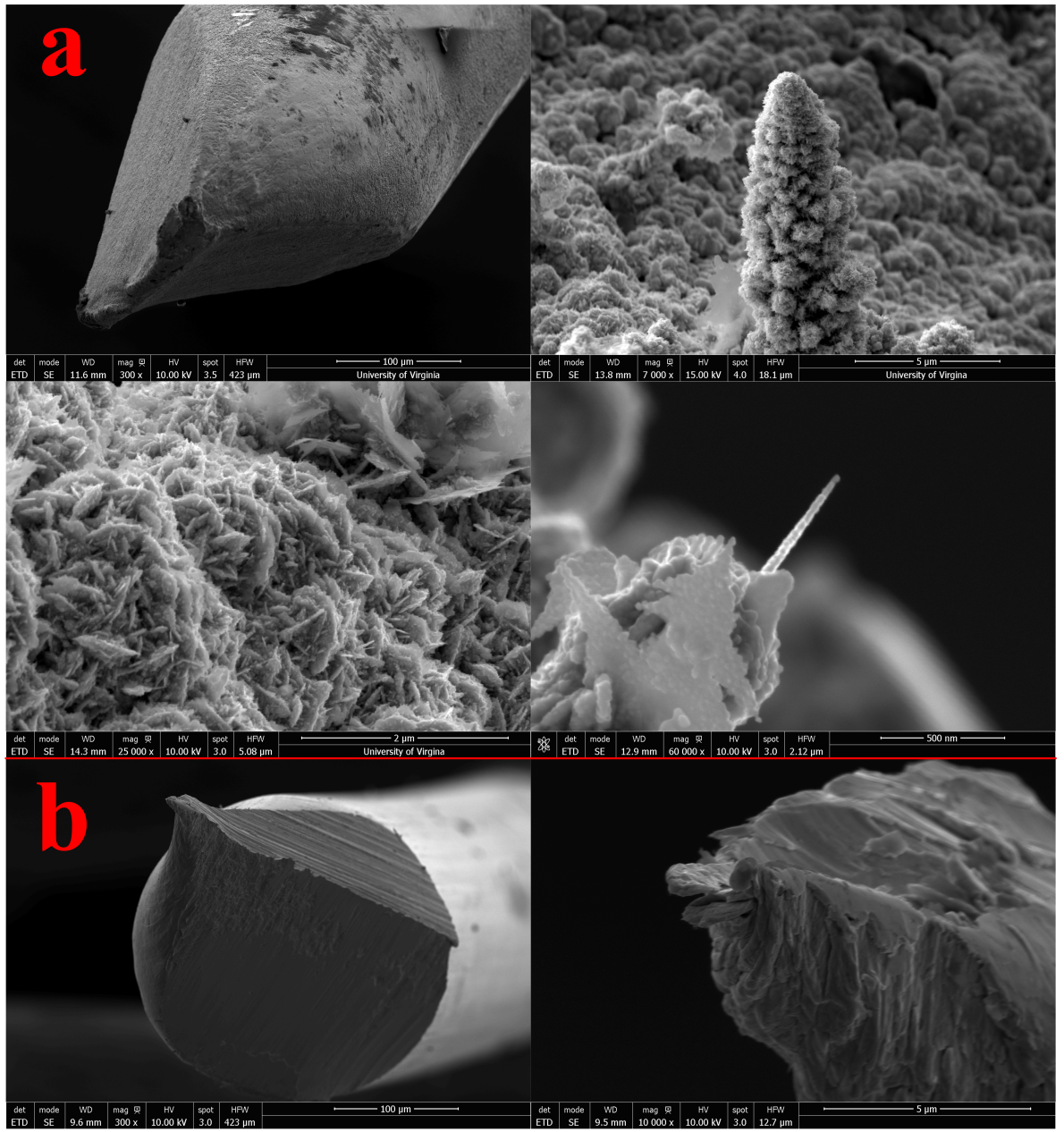

Figure 5.8: SEM images of a) a typical Au nano-structure coated silver wire and b) a typical Pt STM-cut wire. 


\section{Chapter 6}

\section{Conclusion and Outlook}

In this dissertation, field ionization of Rydberg atoms and field emission from metallic nano-tips induced by intense, single-cycle $\mathrm{THz}$ pulses are studied. This chapter reviews the main results of work done in the dissertation, which are presented in detail in the proceeding three chapters. Possibilities for future study are briefly discussed in this chapter as well.

In Chap. 3, we have explored strong-field ionization of excited atoms using singlecycle $\mathrm{THz}$ pulses. We find that adiabatic over-the-barrier ionization is suppressed due to the extended times required for Rydberg electrons to leave the field-dressed potential barrier, resulting in a novel $F \propto n^{-3}$ ionization threshold field scaling. A similar suppression might influence strong-field ionization of delocalized electrons from large molecules. We have also shown that, in contrast to ionization in multicycle fields, in the single-cycle limit, electrons that are ionized near the field extrema acquire substantial energies $\left(\sim 2 U_{p}\right)$, and those ionized near the zero-crossing can obtain much larger energies $\left(>6 U_{p}\right)$, even in the absence of rescattering. We have also briefly reviewed a theoretical extension of our work (by Yang and Robicheaux) that shows other interesting features of strong-field ionization in single-cycle limit, 
such as displacement ionization in the short-pulse regime, and a predicted left-right asymmetry of electron emission. As experimentally available optical pulses approach the single-cycle limit, the physics underlying our results will impact the interpretation and feasibility of measurements involving ground-state atoms and molecules, particularly those where distinguishing recollision from directly ionized electrons is important, or where mapping electron energy to ionization time is desired.

In Chap. 4, we have employed intense, single-cycle $\mathrm{THz}$ pulses to explore the ionization of oriented atoms. The ionization threshold field shows a pronounced variation among states with nearly identical binding energies, but with permanent dipole moments which differ in amplitude and/or direction. This orientation dependence can be attributed to diabatic population transfer between "uphill" and "downhill" oriented states and an asymmetry in the peak $\mathrm{THz}$ field strength in the forward and backward directions. Following that study, we have also discussed the possibility of extending laser-driven electron rescattering into the $\mathrm{THz}$ frequency regime. One motivation for driving electron rescattering with the low-frequency/long-wavelength $\mathrm{THz}$ fields is to scale up the energy of recollision electrons without influencing the ionic core. In order to increase the recollision probability, we consider preparing the atom in a highly excited and oriented Rydberg-Stark state prior to $\mathrm{THz}$ exposure. As a result, initially the electron is (nearly) at rest and located far away from the ionic core, the recollision is confined to one direction and the recollision wavepacket is well characterized. We believe that new sources which produce intense $\mathrm{THz}$ radiation with field strengths on the order of 10-100 MV/cm, or exploiting the $>10 \mathrm{MV} / \mathrm{cm}$ enhanced field in the vicinity of nano- or micro-tipped wire, may get us into this interesting regime.

In Chap. 5, we describe our use of strong, single-cycle THz fields to induce electron emission from tungsten nano-tips. Electrons with energies easily exceeding $5 \mathrm{keV}$ are 
observed, substantially greater than those previously observed using $\mathrm{THz}$, infrared, or near-infrared driving fields. The maximum electron energies are proportional to the peak THz field and are roughly independent of tip radius, in good agreement with the predictions of a sub-cycle energy transfer model. Accordingly, the longer wavelength used in this work, as compared to previous laser induced electron emission studies, appears to be the primary factor responsible for the significantly higher electron energies. Following a full characterization of their temporal and angular distributions, these isolated high-energy electron bursts might serve as sources to enable a variety of applications, including time-resolved electron diffraction. In addition, in this long wavelength regime, the enhanced THz fields in the vicinity of tips with radii, $R>1$ $\mu \mathrm{m}$, while insufficient to induce electron emission, could be employed to explore the influence of large quasi-static fields on HHG, attosecond pulse generation, and other phenomena based on electron rescattering in an intense laser focused near the tip. The study in Chap. 5 mainly focuses on the post-emission energy transfer dynamics, future measurements, such as the absolute emission current, the emission pattern, etc., may provide us information encoded in the emission process. In addition, we have also showed some preliminary experimental results of THz-induced field electron emission from randomly aligned and sized Au multi-nanotips and Pt STM-cut wires, the results are interesting but not fully understood. Further study on different materials and/or structures with well characterized geometric shapes may help us better explore field emission in the $\mathrm{THz}$ frequency regime. 


\section{Bibliography}

[1] G. D. Liveing and J. Dewar. On the spectra of sodium and potassium. Proc. R. Soc. Lond., 29(196-199):398-402, 1879. doi:10.1098/rspl.1879.0067. (Cited on p. 2)

[2] W. N. Hartley. On homologous spectra. J. Chem. Soc., Trans., 43:390-400, 1883. doi:10.1039/CT8834300390. (Cited on p. 2)

[3] J. J. Balmer. Notiz über die spectrallinien des wasserstoffs. Annalen der Physik, 261(5):80-87, 1885. doi:10.1002/andp.18852610506. (Cited on p. 2)

[4] J. R. Rydberg. On the structure of the line-spectra of the chemical elements. Phil. Mag. 5th Ser., 29(179):331-337, 1890. doi:10.1080/14786449008619945. (Cited on p. 2)

[5] N. Bohr. On the constitution of atoms and molecules. Phil. Mag. 6th Ser., 26 (151):1-25, 1913. doi:10.1080/14786441308634955. (Cited on p. 3)

[6] David J. Griffiths. Introduction to Quantum Mechanics. Prentice Hall, first edition, 1994. (Cited on p. 3)

[7] L. D. Landau and E. M. Lifshitz. Mechanics, Volume 1. ButterworthHeinemann, third edition, 1976. (Cited on p. 4)

[8] Bernd Thaller. Advanced Visual Quantum Mechanics. Springer, first edition, 2005. (Cited on p. 4)

[9] Gordon W. F. Drake et al. Springer Handbook of Atomic, Molecular, and Optical Physics. Springer, first edition, 2006. (Cited on p. 5)

[10] Thomas F. Gallagher. Rydberg Atoms. Cambridge University Press, first edition, 1994. (Cited on p. 6, 7, 8, 9, 10, 12, 15, 58, 59, 60, 61, 62, 71, 84, 155)

[11] R. F. Stebbings and F. B. Dunning et al. Rydberg States of Atoms and Molecules. Cambridge University Press, first edition, 1983. (Cited on p. 7, $9,11)$ 
[12] M J Seaton. Quantum defect theory. Reports on Progress in Physics, 46(2): 167-257, 1983. doi:10.1088/0034-4885/46/2/002. (Cited on p. 7)

[13] U. FANO and J. W. COOPER. Spectral distribution of atomic oscillator strengths. Rev. Mod. Phys., 40:441-507, Jul 1968. doi:10.1103/RevModPhys.40.441. (Cited on p. 11)

[14] G. S. Voronov and N. B. Delone. Ionization of the xenon atom by the electric field of ruby laser emission. JETP Letters, 1(2):66-68, 1965. http://www . jetpletters.ac.ru/ps/1590/article_24398.shtml. (Cited on p. 14)

[15] P. Agostini, F. Fabre, G. Mainfray, G. Petite, and N. K. Rahman. Free-free transitions following six-photon ionization of xenon atoms. Phys. Rev. Lett., 42:1127-1130, Apr 1979. doi:10.1103/PhysRevLett.42.1127. (Cited on p. 14)

[16] M. V. Ammosov, N. B. Delone, and V. P. Krainov. Tunnel ionization of complex atoms and of atomic ions in an alternating electromagnetic field. Sov. Phys. JETP, 64(6):1191-1194, 1986. http://www. jetp.ac.ru/cgi-bin/e/index/e/ 64/6/p1191?a=list. (Cited on p. 15)

[17] L. V. Keldysh. Ionization in the field of a strong electromagnetic wave. Sov. Phys. JETP, 20(5):1307-1314, 1965. http://www.jetp.ac.ru/cgi-bin/e/ index/e/20/5/p1307?a=list. (Cited on p. 15, 94)

[18] M Protopapas, C H Keitel, and P L Knight. Atomic physics with superhigh intensity lasers. Reports on Progress in Physics, 60(4):389-486, 1997. doi:10.1088/0034-4885/60/4/001. (Cited on p. 18)

[19] P. B. Corkum. Plasma perspective on strong field multiphoton ionization. Phys. Rev. Lett., 71:1994-1997, Sep 1993. doi:10.1103/PhysRevLett.71.1994. (Cited on p. 19, 65)

[20] K. C. Kulander, K. J. Schafer, and J. L. Krause. in Super-Intense LaserAtom Physics, NATO ASI, Ser. B: Physics, Vol. 316, edited by B. Piraux, A. L'Huillier and K. Rzazewski. Plenum Press New York, first edition, 1993.

[21] K. J. Schafer, Baorui Yang, L. F. DiMauro, and K. C. Kulander. Above threshold ionization beyond the high harmonic cutoff. Phys. Rev. Lett., 70:1599-1602, Mar 1993. doi:10.1103/PhysRevLett.70.1599. (Cited on p. 19)

[22] H. B. Van Linden van den Heuvell and H. G. Muller. in Multiphoton Processes, edited by S. J. Smith and P. L. Knight. Cambridge University Press, first edition, 1988. (Cited on p. 20, 58, 68)

[23] T. F. Gallagher. Above-threshold ionization in low-frequency limit. Phys. Rev. Lett., 61:2304-2307, Nov 1988. doi:10.1103/PhysRevLett.61.2304. (Cited on p. 135) 
[24] P. B. Corkum, N. H. Burnett, and F. Brunel. Above-threshold ionization in the long-wavelength limit. Phys. Rev. Lett., 62:1259-1262, Mar 1989. doi:10.1103/PhysRevLett.62.1259. (Cited on p. 20, 58, 68)

[25] G. G. Paulus, W. Becker, and H. Walther. Classical rescattering effects in two-color above-threshold ionization. Phys. Rev. A, 52:4043-4053, Nov 1995. doi:10.1103/PhysRevA.52.4043. (Cited on p. 20)

[26] H. Hertz. Ueber einen einfluss des ultravioletten lichtes auf die electrische entladung. Annalen der Physik, 267(8):983-1000, 1887. doi:10.1002/andp.18872670827. (Cited on p. 22)

[27] A. Einstein. Über einen die erzeugung und verwandlung des lichtes betreffenden heuristischen gesichtspunkt. Annalen der Physik, 322(6):132-148, 1905. doi:10.1002/andp.19053220607. (Cited on p. 22)

[28] R. H. Fowler and L. Nordheim. Electron emission in intense electric fields. Proc. R. Soc. Lond. A, 119(781):173-181, 1928. doi:10.1098/rspa.1928.0091. (Cited on p. $22,100,104,159)$

[29] E. W. Müller. FEM. Zeitschrift für technische Physik, 17:412, 1936. (Cited on p. 22)

[30] Michael Krüger. Attosecond Physics in Strong-Field Photoemission from Metal Nanotips. PhD thesis, Ludwig-Maximilians-Universität München, 2013. (Cited on p. 22)

[31] E. L. Murphy and R. H. Good. Thermionic emission, field emission, and the transition region. Phys. Rev., 102:1464-1473, Jun 1956. doi:10.1103/PhysRev.102.1464. (Cited on p. 23)

[32] Richard G. Forbes. Physics of generalized Fowler-Nordheim-type equations. Journal of Vacuum Science and Technology B, 26(2):788-793, 2008. doi:10.1116/1.2827505. (Cited on p. 23)

[33] Richard G Forbes and Jonathan H.B Deane. Reformulation of the standard theory of Fowler-Nordheim tunnelling and cold field electron emission. Proc. $R$. Soc. Lond. A, 463(2087):2907-2927, 2007. doi:10.1098/rspa.2007.0030. (Cited on p. 24)

[34] M. K. Miller, A. Cerezo, M. G. Hetherington, and G. D. W. Smith. Atom Probe Field Ion Microscopy. Clarendon Press, first edition, 1996. (Cited on p. 25, 104)

[35] S. PODENOK, M. SVEnINGSSON, K. HANSEN, and E. E. B. CAMPBELL. Electric field enhancement factors around a metallic, end-capped cylinder. Nano, 01(01):87-93, 2006. doi:10.1142/S1793292006000112. (Cited on p. 25, $103,104)$ 
[36] Yun-Shik Lee. Principles of Terahertz Science and Technology. Springer US, first edition, 2009. (Cited on p. 26, 27, 28, 29, 30)

[37] Xi-Cheng Zhang and Jingzhou Xu. Introduction to THz Wave Photonics. Springer US, first edition, 2010. (Cited on p. 26, 27, 28)

[38] Gerard Mourou, Charles V. Stancampiano, and Daniel Blumenthal. Picosecond microwave pulse generation. Appl. Phys. Lett., 38(6):470-472, 1981. doi:10.1063/1.92407. (Cited on p. 26)

[39] D. H. Auston, K. P. Cheung, and P. R. Smith. Picosecond photoconducting hertzian dipoles. Appl. Phys. Lett., 45(3):284-286, 1984. doi:10.1063/1.95174.

[40] D. H. Auston, K. P. Cheung, J. A. Valdmanis, and D. A. Kleinman. Cherenkov radiation from femtosecond optical pulses in electro-optic media. Phys. Rev. Lett., 53:1555-1558, Oct 1984. doi:10.1103/PhysRevLett.53.1555. (Cited on p. 35)

[41] D. Grischkowsky, I. N. Duling III, J. C. Chen, and C. C. Chi. Electromagnetic shock waves from transmission lines. Phys. Rev. Lett., 59:1663-1666, Oct 1987. doi:10.1103/PhysRevLett.59.1663.

[42] D. H. Auston and M. C. Nuss. Electrooptical generation and detection of femtosecond electrical transients. IEEE Journal of Quantum Electronics, 24 (2):184-197, Feb 1988. doi:10.1109/3.114.

[43] Ch. Fattinger and D. Grischkowsky. Point source Terahertz optics. Appl. Phys. Lett., 53(16):1480-1482, 1988. doi:10.1063/1.99971.

[44] Ch. Fattinger and D. Grischkowsky. Terahertz beams. Appl. Phys. Lett., 54(6): 490-492, 1989. doi:10.1063/1.100958.

[45] X.C. Zhang, et al. . Terahertz optical rectification from a nonlinear organic crystal. Appl. Phys. Lett., 61(26):3080-3082, 1992. doi:10.1063/1.107968.

[46] X.C. Zhang, B. B. Hu, J. T. Darrow, and D. H. Auston. Generation of femtosecond electromagnetic pulses from semiconductor surfaces. Appl. Phys. Lett., 56 (11):1011-1013, 1990. doi:10.1063/1.102601.

[47] Q. Wu and X.C. Zhang. Freespace electrooptic sampling of Terahertz beams. Appl. Phys. Lett., 67(24):3523-3525, 1995. doi:10.1063/1.114909. (Cited on p. 26)

[48] Klaus Reimann. Table-top sources of ultrashort THz pulses. Reports on Progress in Physics, 70(10):1597-1632, 2007. doi:10.1088/0034-4885/70/10/R02. (Cited on p. 27) 
[49] Matthias C Hoffmann and József András Fülöp. Intense ultrashort Terahertz pulses: generation and applications. J. Phys. D: Appl. Phys., 44(8):083001, 2011. doi:10.1088/0022-3727/44/8/083001. (Cited on p. 27)

[50] Tobias Kampfrath, Koichiro Tanaka, and Keith A. Nelson. Resonant and nonresonant control over matter and light by intense Terahertz transients. Nat. Photon., 7:680-690, Aug 2013. doi:10.1038/nphoton.2013.184. (Cited on p. 28)

[51] Masayoshi Tonouchi. Cutting-edge Terahertz technology. Nat. Photon., 1:97105, Feb 2007. doi:10.1038/nphoton.2007.3. (Cited on p. 28)

[52] D. E. Spence, P. N. Kean, and W. Sibbett. 60-fsec pulse generation from a self-mode-locked Ti:sapphire laser. Opt. Lett., 16(1):42-44, Jan 1991. doi:10.1364/OL.16.000042. (Cited on p. 32)

[53] U. Morgner, et al. . Sub-two-cycle pulses from a Kerr-lens modelocked Ti:sapphire laser. Opt. Lett., 24(6):411-413, Mar 1999. doi:10.1364/OL.24.000411. (Cited on p. 32)

[54] R. Ell, et al. . Generation of 5-fs pulses and octave-spanning spectra directly from a Ti:sapphire laser. Opt. Lett., 26(6):373-375, Mar 2001. doi:10.1364/OL.26.000373. (Cited on p. 32)

[55] Donna Strickland and Gerard Mourou. Compression of amplified chirped optical pulses. Opt. Commun., 55(6):447-449, 1985. doi:10.1016/0030-4018(85)901518. (Cited on p. 32)

[56] Jeremy J. Murray-Krezan. Probing Stark Atoms. PhD thesis, University of Virginia, 2007. (Cited on p. 33)

[57] Xiangdong Zhang. Probing Time-Dependent Electron Interactions in DoubleRydberg Wave packets. PhD thesis, University of Virginia, 2008. (Cited on p. 33)

[58] Mukesh Jewariya, Masaya Nagai, and Koichiro Tanaka. Enhancement of Terahertz wave generation by cascaded $\chi^{(2)}$ processes in $\mathrm{LiNbO}_{3}$. J. Opt. Soc. Am. B, 26(9):A101-A106, Sep 2009. doi:10.1364/JOSAB.26.00A101. (Cited on p. 34)

[59] János Hebling, Ka-Lo Yeh, Matthias C. Hoffmann, Balázs Bartal, and Keith A. Nelson. Generation of high-power Terahertz pulses by tilted-pulse-front excitation and their application possibilities. J. Opt. Soc. Am. B, 25(7):B6-B19, Jul 2008. doi:10.1364/JOSAB.25.0000B6. (Cited on p. 35, 36, 38, 66, 81)

[60] D. H. Auston. Subpicosecond electrooptic shock waves. Appl. Phys. Lett., 43 (8):713-715, 1983. doi:10.1063/1.94486. (Cited on p. 35) 
[61] D. Kleinman and D. Auston. Theory of electrooptic shock radiation in nonlinear optical media. IEEE Journal of Quantum Electronics, 20(8):964-970, August 1984. doi:10.1109/JQE.1984.1072489. (Cited on p. 35)

[62] Y.-S. Lee, et al. . Generation of narrow-band Terahertz radiation via optical rectification of femtosecond pulses in periodically poled lithium niobate. Appl. Phys. Lett., 76(18):2505-2507, 2000. doi:10.1063/1.126390. (Cited on p. 35)

[63] J.A. L'huillier, G. Torosyan, M. Theuer, Y. Avetisyan, and R. Beigang. Generation of $\mathrm{THz}$ radiation using bulk, periodically and aperiodically poled lithium niobate - Part 1: Theory. Appl. Phys. B, 86(2):185-196, 2006. doi:10.1007/s00340-006-2490-9.

[64] J.A. L'huillier, et al. . Generation of THz radiation using bulk, periodically and aperiodically poled lithium niobate - Part 2: Experiments. Appl. Phys. B, 86 (2):197-208, 2006. doi:10.1007/s00340-006-2528-z. (Cited on p. 35)

[65] János Hebling, Gábor Almási, Ida Z. Kozma, and Jürgen Kuhl. Velocity matching by pulse front tilting for large-area THz-pulse generation. Opt. Express, 10 (21):1161-1166, Oct 2002. doi:10.1364/OE.10.001161. (Cited on p. 35)

[66] J. A. Fülöp, L. Pálfalvi, G. Almási, and J. Hebling. Design of high-energy Terahertz sources based on optical rectification. Opt. Express, 18(12):1231112327, Jun 2010. doi:10.1364/OE.18.012311. (Cited on p. 35, 38)

[67] József András Fülöp, László Pálfalvi, Matthias C. Hoffmann, and János Hebling. Towards generation of mJ-level ultrashort $\mathrm{THz}$ pulses by optical rectification. Opt. Express, 19(16):15090-15097, Aug 2011. doi:10.1364/OE.19.015090. (Cited on p. 36)

[68] Michael I. Bakunov, Sergey B. Bodrov, and Eugene A. Mashkovich. Terahertz generation with tilted-front laser pulses: dynamic theory for lowabsorbing crystals. J. Opt. Soc. Am. B, 28(7):1724-1734, Jul 2011. doi:10.1364/JOSAB.28.001724.

[69] H. Hirori, A. Doi, F. Blanchard, and K. Tanaka. Single-cycle Terahertz pulses with amplitudes exceeding $1 \mathrm{MV} / \mathrm{cm}$ generated by optical rectification in LiNbO $_{3}$. Appl. Phys. Lett., 98(9):091106, 2011. doi:10.1063/1.3560062. (Cited on p. $38,66,81)$

[70] Shu-Wei Huang, et al. . High conversion efficiency, high energy Terahertz pulses by optical rectification in cryogenically cooled lithium niobate. Opt. Lett., 38 (5):796-798, Mar 2013. doi:10.1364/OL.38.000796.

[71] J. A. Fülöp, et al. . Generation of sub-mJ Terahertz pulses by optical rectification. Opt. Lett., 37(4):557-559, Feb 2012. doi:10.1364/OL.37.000557. 
[72] J. A. Fülöp, et al. . Efficient generation of THz pulses with $0.4 \mathrm{~mJ}$ energy. Opt. Express, 22(17):20155-20163, Aug 2014. doi:10.1364/OE.22.020155. (Cited on p. 35,36$)$

[73] J. Hebling. Derivation of the pulse front tilt caused by angular dispersion. Optical and Quantum Electronics, 28(12):1759-1763, 1996. doi:10.1007/BF00698541. (Cited on p. 37)

[74] Sara Casalbuoni, et al. . TESLA Report 2005-01: Numerical studies on the electro-optic sampling of relativistic electron bunches, 2005. (Cited on p. 39, 40)

[75] S. Casalbuoni, et al. . Numerical studies on the electro-optic detection of femtosecond electron bunches. Phys. Rev. ST Accel. Beams, 11:072802, Jul 2008. doi:10.1103/PhysRevSTAB.11.072802. (Cited on p. 39)

[76] Axel Winter. Bunch Length Measurements using Electro-optical Sampling at the SLS Linac. PhD thesis, Deutschen Elektronen-Synchrotron (DESY), 2004. (Cited on p. 40)

[77] Hamamatsu Photonics. Guide to Streak Cameras, . (Cited on p. 43)

[78] Hamamatsu Photonics. Fesca-200 streak camera C6138-11,C6138-12, . (Cited on p. 43)

[79] P. B. Corkum and Ferenc Krausz. Attosecond science. Nat. Phys., 3:381-387, Jun 2007. doi:10.1038/nphys620. (Cited on p. 43, 94)

[80] Ferenc Krausz and Misha Ivanov. Attosecond physics. Rev. Mod. Phys., 81: 163-234, Feb 2009. doi:10.1103/RevModPhys.81.163. (Cited on p. 94)

[81] Ferenc Krausz and Mark I. Stockman. Attosecond metrology: from electron capture to future signal processing. Nat. Photon., 8:205-213, Feb 2014. doi:10.1038/nphoton.2014.28. (Cited on p. 43)

[82] E. Goulielmakis, et al. . Direct measurement of light waves. Science, 305(5688): 1267-1269, Aug 2004. doi:10.1126/science.1100866. (Cited on p. 44, 45, 67, 81)

[83] Ulrike Frühling, et al. . Single-shot Terahertz-field-driven X-ray streak camera. Nat. Photon., 3:523-528, Aug 2009. doi:10.1038/nphoton.2009.160. (Cited on p. $44,67,81)$

[84] Marvin J. Weber. Handbook of Laser Wavelengths. CRC Press, first edition, 1998. (Cited on p. 46) 
[85] Frank J. Duarte. Organic dye lasers: Brief history and recent developments. Opt. Photon. News, 14(10):20-25, Oct 2003. doi:10.1364/OPN.14.10.000020. (Cited on p. 46)

[86] T. W. Hänsch. Repetitively pulsed tunable dye laser for high resolution spectroscopy. Appl. Opt., 11(4):895-898, Apr 1972. doi:10.1364/A0.11.000895. (Cited on p. 46)

[87] Michael G. Littman. Single-mode operation of grazing-incidence pulsed dye laser. Opt. Lett., 3(4):138-140, Oct 1978. doi:10.1364/OL.3.000138. (Cited on p. 46)

[88] Spectra-Physics. Cobra pulsed dye lasers. (Cited on p. 47)

[89] Continuum. Vista narrow linewidth dye lasers. (Cited on p. 47)

[90] J. E. Bayfield and P. M. Koch. Multiphoton ionization of highly excited hydrogen atoms. Phys. Rev. Lett., 33:258-261, Jul 1974. doi:10.1103/PhysRevLett.33.258. (Cited on p. 58)

[91] Theodore W. Ducas, Michael G. Littman, Richard R. Freeman, and Daniel Kleppner. Stark ionization of high-lying states of sodium. Phys. Rev. Lett., 35: 366-369, Aug 1975. doi:10.1103/PhysRevLett.35.366. (Cited on p. 58, 71)

[92] T. F. Gallagher, L. M. Humphrey, R. M. Hill, and S. A. Edelstein. Resolution of $\left|m_{l}\right|$ and $\left|m_{j}\right|$ levels in the electric field ionization of highly excited $d$ states of Na. Phys. Rev. Lett., 37:1465-1467, Nov 1976. doi:10.1103/PhysRevLett.37.1465.

[93] T. H. Jeys, et al. . Diabatic field ionization of highly excited sodium atoms. Phys. Rev. Lett., 44:390-393, Feb 1980. doi:10.1103/PhysRevLett.44.390. (Cited on p. 58, 71)

[94] C. R. Mahon, J. L. Dexter, P. Pillet, and T. F. Gallagher. Ionization of sodium and lithium Rydberg atoms by $10-\mathrm{MHz}$ to $15-\mathrm{GHz}$ electric fields. Phys. Rev. A, 44:1859-1873, Aug 1991. doi:10.1103/PhysRevA.44.1859.

[95] C O Reinhold, M Melles, Hai Shao, and J Burgdorfer. Ionization of Rydberg atoms by half-cycle pulses. J. Phys. B: At. Mol Opt. Phys., 26(19):L659, 1993. doi:10.1088/0953-4075/26/19/005. (Cited on p. 58, 63, 64)

[96] S. Yoshida, et al. . Accurate ionization thresholds of atoms subject to half-cycle pulses. Phys. Rev. A, 58:2229-2241, Sep 1998. doi:10.1103/PhysRevA.58.2229. (Cited on p. 58)

[97] P. Pillet, W. W. Smith, R. Kachru, N. H. Tran, and T. F. Gallagher. Microwave ionization of Na Rydberg levels. Phys. Rev. Lett., 50:1042-1045, Apr 1983. doi:10.1103/PhysRevLett.50.1042. (Cited on p. 58, 62, 63) 
[98] Michael W. Noel, W. M. Griffith, and T. F. Gallagher. Population trapping in extremely highly excited states in microwave ionization. Phys. Rev. Lett., 83: 1747-1750, Aug 1999. doi:10.1103/PhysRevLett.83.1747. (Cited on p. 58)

[99] R. R. Jones, D. You, and P. H. Bucksbaum. Ionization of Rydberg atoms by subpicosecond half-cycle electromagnetic pulses. Phys. Rev. Lett., 70:1236-1239, Mar 1993. doi:10.1103/PhysRevLett.70.1236. (Cited on p. 58, 63, 72)

[100] C. Wesdorp, F. Robicheaux, and L. D. Noordam. Displacing Rydberg electrons: The mono-cycle nature of half-cycle pulses. Phys. Rev. Lett., 87:083001, Aug 2001. doi:10.1103/PhysRevLett.87.083001. (Cited on p. 58, 63, 77)

[101] S. Augst, D. Strickland, D. D. Meyerhofer, S. L. Chin, and J. H. Eberly. Tunneling ionization of noble gases in a high-intensity laser field. Phys. Rev. Lett., 63:2212-2215, Nov 1989. doi:10.1103/PhysRevLett.63.2212. (Cited on p. 58)

[102] M. Lezius, et al. . Nonadiabatic multielectron dynamics in strong field molecular ionization. Phys. Rev. Lett., 86:51-54, Jan 2001. doi:10.1103/PhysRevLett.86.51. (Cited on p. 58)

[103] George N. Gibson. Multiphoton $\pi$ pulses. Phys. Rev. Lett., 89:263001, Dec 2002. doi:10.1103/PhysRevLett.89.263001. (Cited on p. 58)

[104] E. S. Shuman, R. R. Jones, and T. F. Gallagher. Multiphoton assisted recombination. Phys. Rev. Lett., 101:263001, Dec 2008. doi:10.1103/PhysRevLett.101.263001. (Cited on p. 58, 68)

[105] T. F. Gallagher, L. M. Humphrey, W. E. Cooke, R. M. Hill, and S. A. Edelstein. Field ionization of highly excited states of sodium. Phys. Rev. A, 16:1098-1108, Sep 1977. doi:10.1103/PhysRevA.16.1098. (Cited on p. 62)

[106] Turker Topcu and Francis Robicheaux. Dichotomy between tunneling and multiphoton ionization in atomic photoionization: Keldysh parameter $\gamma$ versus scaled frequency $\Omega$. Phys. Rev. A, 86:053407, Nov 2012. doi:10.1103/PhysRevA.86.053407. (Cited on p. 65)

[107] B. Walker, et al. . Precision measurement of strong field double ionization of helium. Phys. Rev. Lett., 73:1227-1230, Aug 1994. doi:10.1103/PhysRevLett.73.1227. (Cited on p. 65, 134)

[108] G. G. Paulus, W. Nicklich, Huale Xu, P. Lambropoulos, and H. Walther. Plateau in above threshold ionization spectra. Phys. Rev. Lett., 72:2851-2854, May 1994. doi:10.1103/PhysRevLett.72.2851. (Cited on p. 65)

[109] R. Kienberger, et al. . Steering attosecond electron wave packets with light. Science, 297(5584):1144-1148, Aug 2002. doi:10.1126/science.1073866. (Cited on p. $67,81,94)$ 
[110] Cosmin I. Blaga, et al. . Imaging ultrafast molecular dynamics with laser-induced electron diffraction. Nature, 483:194-197, Mar 2012. doi:10.1038/nature10820. (Cited on p. 69)

[111] Y. Huismans, et al. . Scaling laws for photoelectron holography in the midinfrared wavelength regime. Phys. Rev. Lett., 109:013002, Jul 2012. doi:10.1103/PhysRevLett.109.013002.

[112] Boris Bergues, et al. . Attosecond tracing of correlated electron-emission in non-sequential double ionization. Nat. Commun., 3:813, May 2012. doi:10.1038/ncomms1807. (Cited on p. 69)

[113] Michael Spanner, Olga Smirnova, Paul B Corkum, and Misha Yu Ivanov. Reading diffraction images in strong field ionization of diatomic molecules. J. Phys. B: At. Mol Opt. Phys., 37(12):L243, 2004. doi:10.1088/0953-4075/37/12/L02. (Cited on p. 69)

[114] Xue-Bin Bian and André D. Bandrauk. Attosecond time-resolved imaging of molecular structure by photoelectron holography. Phys. Rev. Lett., 108:263003, Jun 2012. doi:10.1103/PhysRevLett.108.263003. (Cited on p. 69)

[115] T. Wittmann, et al. . Single-shot carrierenvelope phase measurement of fewcycle laser pulses. Nat. Phys., 5:357-362, Apr 2009. doi:10.1038/nphys1250. (Cited on p. 69)

[116] D. Ray, et al. . Momentum spectra of electrons rescattered from rare-gas targets following their extraction by one- and two-color femtosecond laser pulses. Phys. Rev. A, 83:013410, Jan 2011. doi:10.1103/PhysRevA.83.013410. (Cited on p. 69)

[117] Th. Weber, et al. . Recoil-ion momentum distributions for single and double ionization of helium in strong laser fields. Phys. Rev. Lett., 84:443-446, Jan 2000. doi:10.1103/PhysRevLett.84.443. (Cited on p. 69)

[118] B. Feuerstein, et al. . Separation of recollision mechanisms in nonsequential strong field double ionization of Ar: The role of excitation tunneling. Phys. Rev. Lett., 87:043003, Jul 2001. doi:10.1103/PhysRevLett.87.043003.

[119] N. Camus, et al. . Attosecond correlated dynamics of two electrons passing through a transition state. Phys. Rev. Lett., 108:073003, Feb 2012. doi:10.1103/PhysRevLett.108.073003.

[120] T. Nubbemeyer, K. Gorling, A. Saenz, U. Eichmann, and W. Sandner. Strongfield tunneling without ionization. Phys. Rev. Lett., 101:233001, Dec 2008. doi:10.1103/PhysRevLett.101.233001. (Cited on p. 69) 
[121] Michael G. Littman, Michael M. Kash, and Daniel Kleppner. Fieldionization processes in excited atoms. Phys. Rev. Lett., 41:103-107, Jul 1978. doi:10.1103/PhysRevLett.41.103. (Cited on p. 71)

[122] B. C. Yang and F. Robicheaux. Field-ionization threshold and its induced ionization-window phenomenon for Rydberg atoms in a short single-cycle pulse. Phys. Rev. A, 90:063413, Dec 2014. doi:10.1103/PhysRevA.90.063413. (Cited on p. $75,76,77,158)$

[123] B. C. Yang and F. Robicheaux. Field ionization of Rydberg atoms in a single-cycle pulse. Phys. Rev. A, 91:043407, Apr 2015. doi:10.1103/PhysRevA.91.043407. (Cited on p. 76)

[124] Sha $\mathrm{Li}$ and R. R. Jones. Ionization of excited atoms by intense single-cycle THz pulses. Phys. Rev. Lett., 112:143006, Apr 2014. doi:10.1103/PhysRevLett.112.143006. (Cited on p. 79, 97, 98, 103)

[125] K. N. Egodapitiya, Sha Li, and R. R. Jones. Terahertz-induced field-free orientation of rotationally excited molecules. Phys. Rev. Lett., 112:103002, Mar 2014. doi:10.1103/PhysRevLett.112.103002. (Cited on p. 79)

[126] Lotte Holmegaard, et al. . Photoelectron angular distributions from strongfield ionization of oriented molecules. Nat. Phys., 6:428-432, May 2010. doi:10.1038/nphys1666. (Cited on p. 79)

[127] R. R. Jones, N. E. Tielking, D. You, C. Raman, and P. H. Bucksbaum. Ionization of oriented Rydberg states by subpicosecond halfcycle electromagnetic pulses. Phys. Rev. A, 51:R2687-R2690, Apr 1995. doi:10.1103/PhysRevA.51.R2687. (Cited on p. 79, 80)

[128] L. D. Landau. On the theory of transfer of energy at collisions ii. Phys. Z. Sowjetunion, 2:46-51, 1932. (Cited on p. 84, 154, 155)

[129] Clarence Zener. Non-adiabatic crossing of energy levels. Proc. R. Soc. Lond. A, 137(833):696-702, 1932. doi:10.1098/rspa.1932.0165. (Cited on p. 154)

[130] Jan R. Rubbmark, Michael M. Kash, Michael G. Littman, and Daniel Kleppner. Dynamical effects at avoided level crossings: A study of the LandauZener effect using Rydberg atoms. Phys. Rev. A, 23:3107-3117, Jun 1981. doi:10.1103/PhysRevA.23.3107. (Cited on p. 84, 154, 155)

[131] B. C. Yang and F. Robicheaux. Temporal interferences driven by a single-cycle Terahertz pulse in the photodetachment dynamics of negative ions. Phys. Rev. A, 92:063410, Dec 2015. doi:10.1103/PhysRevA.92.063410. (Cited on p. 89) 
[132] Hiromichi Niikura, et al. . Sub-laser-cycle electron pulses for probing molecular dynamics. Nature, 417:917-922, Jun 2002. doi:10.1038/nature00787. (Cited on p. 94)

[133] M. Drescher, et al. . Time-resolved atomic inner-shell spectroscopy. Nature, 419:803-807, Oct 2002. doi:10.1038/nature01143.

[134] A. L. Cavalieri, et al. . Attosecond spectroscopy in condensed matter. Nature, 449:1029-1032, Oct 2007. doi:10.1038/nature06229.

[135] M. Schultze, et al. . Delay in photoemission. Science, 328(5986):1658-1662, Jun 2010. doi:10.1126/science.1189401.

[136] Eleftherios Goulielmakis, et al. . Real-time observation of valence electron motion. Nature, 466:739-743, Aug 2010. doi:10.1038/nature09212.

[137] M. Meckel, et al. . Laser-induced electron tunneling and diffraction. Science, 320(5882):1478-1482, Jun 2008. doi:10.1126/science.1157980. (Cited on p. 96)

[138] Cosmin I. Blaga, et al. . Imaging ultrafast molecular dynamics with laser-induced electron diffraction. Nature, 483:194-197, Mar 2012. doi:10.1038/nature10820.

[139] Junliang Xu, et al. . Diffraction using laser-driven broadband electron wave packets. Nat. Commun., 5:4635, Aug 2014. doi:10.1038/ncomms5635. (Cited on p. 94, 96)

[140] G. Herink, D. R. Solli, M. Gulde, and C. Ropers. Field-driven photoemission from nanostructures quenches the quiver motion. Nature, 483:190-193, Mar 2012. doi:10.1038/nature10878. (Cited on p. 94, 95, 97, 100, 102, 158)

[141] R. Bormann, M. Gulde, A. Weismann, S. V. Yalunin, and C. Ropers. Tipenhanced strong-field photoemission. Phys. Rev. Lett., 105:147601, Sep 2010. doi:10.1103/PhysRevLett.105.147601.

[142] C. Ropers, D. R. Solli, C. P. Schulz, C. Lienau, and T. Elsaesser. Localized multiphoton emission of femtosecond electron pulses from metal nanotips. Phys. Rev. Lett., 98:043907, Jan 2007. doi:10.1103/PhysRevLett.98.043907.

[143] Michael Krüger, Markus Schenk, and Peter Hommelhoff. Attosecond control of electrons emitted from a nanoscale metal tip. Nature, 475:78-81, Jul 2011. doi:10.1038/nature10196. (Cited on p. 94, 98)

[144] Markus Schenk, Michael Krüger, and Peter Hommelhoff. Strong-field abovethreshold photoemission from sharp metal tips. Phys. Rev. Lett., 105:257601, Dec 2010. doi:10.1103/PhysRevLett.105.257601. 
[145] Peter Hommelhoff, Catherine Kealhofer, and Mark A. Kasevich. Ultrafast electron pulses from a tungsten tip triggered by low-power femtosecond laser pulses. Phys. Rev. Lett., 97:247402, Dec 2006. doi:10.1103/PhysRevLett.97.247402.

[146] Peter Hommelhoff, Yvan Sortais, Anoush Aghajani-Talesh, and Mark A. Kasevich. Field emission tip as a nanometer source of free electron femtosecond pulses. Phys. Rev. Lett., 96:077401, Feb 2006. doi:10.1103/PhysRevLett.96.077401. (Cited on p. 98)

[147] M Krüger, M Schenk, M Frster, and P Hommelhoff. Attosecond physics in photoemission from a metal nanotip. J. Phys. B: At. Mol Opt. Phys., 45(7): 074006, 2012. doi:10.1088/0953-4075/45/7/074006.

[148] Georg Wachter, et al. . Electron rescattering at metal nanotips induced by ultrashort laser pulses. Phys. Rev. B, 86:035402, Jul 2012. doi:10.1103/PhysRevB.86.035402. (Cited on p. 98)

[149] Michael Krüger, et al. . Interaction of ultrashort laser pulses with metal nanotips: a model system for strong-field phenomena. New J. Phys., 14(8):085019, 2012. doi:10.1088/1367-2630/14/8/085019.

[150] Doo Jae Park, et al. . Strong field acceleration and steering of ultrafast electron pulses from a sharp metallic nanotip. Phys. Rev. Lett., 109:244803, Dec 2012. doi:10.1103/PhysRevLett.109.244803. (Cited on p. 95, 98)

[151] Hirofumi Yanagisawa, et al. . Energy distribution curves of ultrafast laserinduced field emission and their implications for electron dynamics. Phys. Rev. Lett., 107:087601, Aug 2011. doi:10.1103/PhysRevLett.107.087601.

[152] Hirofumi Yanagisawa, et al. . Optical control of field-emission sites by femtosecond laser pulses. Phys. Rev. Lett., 103:257603, Dec 2009. doi:10.1103/PhysRevLett.103.257603. (Cited on p. 95)

[153] Sergey Zherebtsov, et al. . Controlled near-field enhanced electron acceleration from dielectric nanospheres with intense few-cycle laser fields. Nat. Phys., 7: 656-662, Apr 2011. doi:10.1038/nphys1983. (Cited on p. 94, 95)

[154] R. Ganter, et al. . Laser-photofield emission from needle cathodes for low-emittance electron beams. Phys. Rev. Lett., 100:064801, Feb 2008. doi:10.1103/PhysRevLett.100.064801. (Cited on p. 94)

[155] Seungchul Kim, et al. . High-harmonic generation by resonant plasmon field enhancement. Nature, 453:757-760, Jun 2008. doi:10.1038/nature07012. (Cited on p. 94) 
[156] In-Yong Park, et al. . Plasmonic generation of ultrashort extreme-ultraviolet light pulses. Nat. Photon., 5:677-681, Oct 2011. doi:10.1038/nphoton.2011.258.

[157] M. Sivis, M. Duwe, B. Abel, and C. Ropers. Extreme-ultraviolet light generation in plasmonic nanostructures. Nat. Phys., 9:304-309, Mar 2013. doi:10.1038/nphys2590. (Cited on p. 94, 98)

[158] F. M. Charbonnier, J. P. Barbour, L. F. Garrett, and W. P. Dyke. Basic and applied studies of field emission at microwave frequencies. Proceedings of the IEEE, 51(7):991-1004, July 1963. doi:10.1109/PROC.1963.2379. (Cited on p. 95)

[159] George N. Fursey. Field emission in a microwave field. Journal of Vacuum Science and Technology B, 13(2):558-565, 1995. doi:10.1116/1.588354. (Cited on p. 95)

[160] L. Wimmer, et al. . Terahertz control of nanotip photoemission. Nat. Phys., 10:432-436, May 2014. doi:10.1038/nphys2974. (Cited on p. 95, 98)

[161] Tyler L. Cocker, et al. . An ultrafast Terahertz scanning tunnelling microscope. Nat. Photon., 7:620-625, Jul 2013. doi:10.1038/nphoton.2013.151. (Cited on p. 95)

[162] G Herink, L Wimmer, and C Ropers. Field emission at Terahertz frequencies: Ac-tunneling and ultrafast carrier dynamics. New J. Phys., 16(12):123005, 2014. doi:10.1088/1367-2630/16/12/123005. (Cited on p. 95, 100)

[163] Krzysztof Iwaszczuk, Maksim Zalkovskij, Andrew C. Strikwerda, and Peter U. Jepsen. Nitrogen plasma formation through Terahertz-induced ultrafast electron field emission. Optica, 2(2):116-123, Feb 2015. doi:10.1364/OPTICA.2.000116. (Cited on p. 95, 96)

[164] Max Gulde, et al. . Ultrafast low-energy electron diffraction in transmission resolves polymer/graphene superstructure dynamics. Science, 345(6193):200204, Jul 2014. doi:10.1126/science.1250658. (Cited on p. 96, 98, 107)

[165] Min-Qi Bao and Anthony F. Starace. Static-electric-field effects on high harmonic generation. Phys. Rev. A, 53:R3723-R3726, Jun 1996. doi:10.1103/PhysRevA.53.R3723. (Cited on p. 96, 107)

[166] A Lohr, W Becker, and M Kleber. High-harmonic generation affected by a static electric field. Laser Physics, 7(3):615-622, 1997.

[167] Bingbing Wang, Xiaofeng Li, and Panming Fu. The effects of a static electric field on high-order harmonic generation. J. Phys. B: At. Mol Opt. Phys., 31(9): 1961, 1998. doi:10.1088/0953-4075/31/9/012. (Cited on p. 96, 107) 
[168] Sebastian Thomas, Michael Krger, Michael Frster, Markus Schenk, and Peter Hommelhoff. Probing of optical near-fields by electron rescattering on the 1 nm scale. Nano Lett., 13(10):4790-4794, 2013. doi:10.1021/nl402407r. PMID: 24032432. (Cited on p. 103)

[169] J. H. Kang, D. S. Kim, and Q-Han Park. Local capacitor model for plasmonic electric field enhancement. Phys. Rev. Lett., 102:093906, Mar 2009. doi:10.1103/PhysRevLett.102.093906. (Cited on p. 104)

[170] Sebastian Thomas, Georg Wachter, Christoph Lemell, Joachim Burgdörfer, and Peter Hommelhoff. Large optical field enhancement for nanotips with large opening angles. New J. Phys., 17(6):063010, 2015. doi:10.1088/13672630/17/6/063010. (Cited on p. 105)

[171] Johannes Hoffrogge, et al. . Tip-based source of femtosecond electron pulses at 30kev. J. Appl. Phys., 115(9):094506, 2014. doi:10.1063/1.4867185. (Cited on p. 107)

[172] Emilio A. Nanni, et al. . Terahertz-driven linear electron acceleration. Nat. Commun., 6:8486, Oct 2015. doi:10.1038/ncomms9486. (Cited on p. 107)

[173] D. N. Fittinghoff, P. R. Bolton, B. Chang, and K. C. Kulander. Observation of nonsequential double ionization of helium with optical tunneling. Phys. Rev. Lett., 69:2642-2645, Nov 1992. doi:10.1103/PhysRevLett.69.2642. (Cited on p. 134)

[174] P. H. Bucksbaum, R. R. Freeman, M. Bashkansky, and T. J. McIlrath. Role of the ponderomotive potential in above-threshold ionization. J. Opt. Soc. Am. B, 4(5):760-764, May 1987. doi:10.1364/JOSAB.4.000760. (Cited on p. 135)

[175] F. Salin, P. Georges, G. Roger, and A. Brun. Single-shot measurement of a 52fs pulse. Appl. Opt., 26(21):4528-4531, Nov 1987. doi:10.1364/AO.26.004528. (Cited on p. 138) 
Appendices 


\section{Appendix A}

\section{Atomic units}

In the atomic units (a. u.) system, the fundamental constants of elementary charge $e$, electron rest mass $m_{e}$, reduced Planck constant $\hbar$ and Coulomb's constant $k_{e}=$ $1 /\left(4 \pi \varepsilon_{0}\right)$ are defined to be unity: $e=m_{e}=\hbar=k_{e} \equiv 1$. This system is very convenient for atomic physics calculations. Table A.1 lists the values of some important fundamental constants in atomic and SI units.

Table A.1: Values of fundamental constants in atomic and SI units. From 2014 NIST CODATA.

\begin{tabular}{|c|c|c|c|}
\hline Name & Symbol & Value in SI & Value in a. $u$. \\
\hline Elementary charge & $e$ & $1.6021766208(98) \times 10^{-19} \mathrm{C}$ & 1 \\
\hline Electron rest mass & $m_{e}$ & $9.10938356(11) \times 10^{-31} \mathrm{~kg}$ & 1 \\
\hline Reduced Planck constant & $\hbar$ & $1.054571800(13) \times 10^{-34} \mathrm{~J} \cdot \mathrm{s}$ & 1 \\
\hline Electric constant & $\varepsilon_{0}$ & $8.854187817 \ldots \times 10^{-12} \mathrm{~F} \cdot \mathrm{m}^{-1}$ & $1 /(4 \pi)$ \\
\hline Fine structure constant & $\alpha=e^{2} /\left(4 \pi \varepsilon_{0} \hbar c\right)$ & $7.2973525664(17) \times 10^{-3}$ & same as in SI \\
\hline Speed of light in vacuum & $c$ & $2.99792458 \times 10^{8} \mathrm{~m} \cdot \mathrm{s}^{-1}$ & $1 / \alpha=137.035999139(31)$ \\
\hline Rydberg constant & $R_{\infty}=\alpha^{2} m_{e} c /(4 \pi \hbar)$ & $10973731.568508(65) \mathrm{m}^{-1}$ & $R y_{\infty}=2 \pi \hbar c R_{\infty}=1 / 2$ \\
\hline
\end{tabular}

The convenience of using atomic units is particularly clear when describing the classical Bohr orbital motion of the ground state hydrogen atom $\mathrm{H}_{1 s}$. Table A.2 is a list of some derived atomic units, their physical relevance to the Bohr motion of $\mathrm{H}_{1 s}$ 
and conversion constants to SI units.

Table A.2: Derived atomic units. From 2014 NIST CODATA.

\begin{tabular}{|c|c|c|}
\hline 1 a. u. of & Expression & Value in SI \\
\hline Length & $\begin{array}{c}4 \pi \varepsilon_{0} \hbar^{2} /\left(m_{e} e^{2}\right)=\hbar /\left(m_{e} c \alpha\right) \equiv a_{0} \\
\text { Bohr orbital radius of } e\left(\mathrm{H}_{1 s}\right)\end{array}$ & $5.2917721067(12) \times 10^{-11} \mathrm{~m}$ \\
\hline $\begin{array}{c}\text { Energy } \\
\text { (Hartree energy) }\end{array}$ & $\begin{array}{c}e^{2} /\left(4 \pi \varepsilon_{0} a_{0}\right)=\alpha^{2} m_{e} c^{2} \equiv E_{h} \equiv 2 R y_{\infty} \\
\text { Potential energy of } e\left(\mathrm{H}_{1 s}\right) \\
2 \times \text { binging energy of } e\left(\mathrm{H}_{1 s}\right)\end{array}$ & $\begin{array}{c}4.359744650(54) \times 10^{-18} \mathrm{~J} \\
2.721138602(17) \times 10^{1} \mathrm{eV} \\
6.579683920711(39) \times 10^{15} \mathrm{~Hz} \\
2.194746313702(13) \times 10^{7} \mathrm{~m}^{-1} \\
3.1577513(18) \times 10^{5} \mathrm{~K} \\
4.850870129(60) \times 10^{-35} \mathrm{Kg}\end{array}$ \\
\hline Electric field & $\begin{array}{c}E_{h} /\left(e a_{0}\right) \\
\text { Electric field } \\
a_{0} \text { away from proton }\end{array}$ & $5.142206707(32) \times 10^{11} \mathrm{~V} \cdot \mathrm{m}^{-1}$ \\
\hline Force & $\begin{array}{l}\qquad E_{h} / a_{0} \\
\text { Electric force on electron } \\
a_{0} \text { away from proton }\end{array}$ & $8.23872336(10) \times 10^{-8} \mathrm{~N}$ \\
\hline Electric dipole moment & $\begin{array}{l}\qquad e a_{0} \\
\text { Electric dipole moment of electron } \\
a_{0} \text { away from proton }\end{array}$ & $8.478353552(52) \times 10^{-30} \mathrm{C} \cdot \mathrm{m}$ \\
\hline Time & $\begin{array}{c}\hbar / E_{h} \\
(2 \pi)^{-1} \times \text { Orbital period of } e\left(\mathrm{H}_{1 s}\right)\end{array}$ & $2.418884326509(14) \times 10^{-17} \mathrm{~s}$ \\
\hline Velocity & $\begin{array}{c}a_{0} E_{h} / \hbar=\alpha c \\
\text { Radial velocity of } e\left(\mathrm{H}_{1 s}\right)\end{array}$ & $2.18769126277(50) \times 10^{6} \mathrm{~m} \cdot \mathrm{s}^{-1}$ \\
\hline
\end{tabular}




\section{Appendix B}

\section{Coordinates}

Three commonly used coordinate systems in atomic physics are the Cartesian $(x, y, z)$, spherical $(r, \theta, \phi)$ and parabolic coordinates $(\xi, \eta, \phi)$. The relationship between these three coordinates are,

$$
\begin{gathered}
x=r \sin \theta \cos \phi, y=r \sin \theta \sin \phi, z=r \cos \theta \\
x=\sqrt{\xi \eta} \cos \phi, y=\sqrt{\xi \eta} \sin \phi, z=\frac{1}{2}(\xi-\eta) \\
r=\frac{1}{2}(\xi+\eta), \theta=\arccos \frac{\xi-\eta}{\xi+\eta}, \phi_{\text {para }}=\phi_{\text {spher }}
\end{gathered}
$$

The infinitesimal volume element in these three coordinates are

$$
\mathrm{d} V=\mathrm{d} x \mathrm{~d} y \mathrm{~d} z=r^{2} \sin \theta \mathrm{d} r \mathrm{~d} \theta \mathrm{d} \phi=\frac{\xi+\eta}{4} \mathrm{~d} \xi \mathrm{d} \eta \mathrm{d} \phi
$$

The Laplace operator $\nabla^{2}$ in these three coordinates can be expressed as

$$
\begin{aligned}
\nabla^{2} & =\frac{\partial^{2}}{\partial x^{2}}+\frac{\partial^{2}}{\partial y^{2}}+\frac{\partial^{2}}{\partial z^{2}} \\
& =\frac{1}{r^{2}} \frac{\partial}{\partial r}\left(r^{2} \frac{\partial}{\partial r}\right)+\frac{1}{r^{2} \sin \theta} \frac{\partial}{\partial \theta}\left(\sin \theta \frac{\partial}{\partial \theta}\right)+\frac{1}{r^{2} \sin ^{2} \theta} \frac{\partial^{2}}{\partial \phi^{2}} \\
& =\frac{4}{\xi+\eta}\left(\frac{\partial}{\partial \xi}+\xi \frac{\partial^{2}}{\partial \xi^{2}}+\frac{\partial}{\partial \eta}+\eta \frac{\partial^{2}}{\partial \eta^{2}}\right)+\frac{1}{\xi \eta} \frac{\partial^{2}}{\partial \phi^{2}}
\end{aligned}
$$




\section{Appendix $\mathrm{C}$}

\section{Kinematics of rescattering}

If an electron is "born" at rest at time $t_{0}$ in an electric field with vector potential $\mathbf{A}(t)$, its velocity is described by,

$$
\mathbf{v}(t)=\mathbf{p}+\mathbf{A}(t)
$$

here $\mathbf{p}=-\mathbf{A}\left(t_{0}\right)$ is the conserved canonical momentum, a.k.a. the drift momentum in the field. The trajectory of the electron can be found by integrating its velocity,

$$
\mathbf{r}(t)=\int_{t_{0}}^{t} \mathbf{v}\left(t^{\prime}\right) \mathrm{d} t^{\prime}=\int_{t_{0}}^{t} \mathbf{A}\left(t^{\prime}\right) \mathrm{d} t^{\prime}-\mathbf{A}\left(t_{0}\right)\left(t-t_{0}\right)
$$

If the electron never returns to the core, no rescattering occurs, and at the end of the laser pulse the electron has obtained an energy,

$$
E_{d}=\frac{\left[\mathbf{v}\left(t_{\infty}\right)\right]^{2}}{2}=\frac{\left[-\mathbf{A}\left(t_{0}\right)+\mathbf{A}\left(t_{\infty}\right)\right]^{2}}{2}=\frac{\mathbf{A}^{2}\left(t_{0}\right)}{2} \in\left[0,2 U_{p}\right]
$$

Here we have assumed, without loss of generality, that $\mathbf{A}\left(t_{\infty}\right)=0$.

In the rescattering process, the return time of the electron $t_{r}$ can be calculated from $r\left(t_{r}\right)=0$. And knowing the return time, the velocity at the time of return can 
be found,

$$
\mathbf{v}_{r}\left(t_{r}\right)=-\mathbf{A}\left(t_{0}\right)+\mathbf{A}\left(t_{r}\right)
$$

and the returned electron has an energy,

$$
E_{r}=\frac{1}{2}\left[\mathbf{A}\left(t_{r}\right)-\mathbf{A}\left(t_{0}\right)\right]^{2} \in\left[\begin{array}{ll}
0,3.17 U_{p}
\end{array}\right]
$$

The returned electron may recombine with the ionic core, releasing all its kinetic energy plus the ionization potential to HHG,

$$
E_{H H G}=E_{r}+I P
$$

It may also "kick out" another electron(s), provided that it returns with an energy higher than the binding energy of the ejected electron(s). This process is known as non-sequential double (or multiple) ionization (NSDI) $[107,173]$. The returned electron can experience elastic scattering as well, during which its energy is conserved. At the maximum scattering angle of $180^{\circ}$ (backscattering), the magnitude of the velocity does not change, but the electron obtains an extra amount of drift momentum, $-\mathbf{A}\left(t_{r}\right)$, upon backscattering.

$$
\begin{gathered}
\mathbf{v}_{b s}\left(t_{r}\right)=-\mathbf{v}_{r}\left(t_{r}\right)=\mathbf{A}\left(t_{0}\right)-\mathbf{A}\left(t_{r}\right) \\
\mathbf{v}_{b s}\left(t>t_{r}\right)=\mathbf{v}_{b s}\left(t_{r}\right)-\mathbf{A}\left(t_{r}\right)+\mathbf{A}(t)=\mathbf{A}\left(t_{0}\right)-2 \mathbf{A}\left(t_{r}\right)+\mathbf{A}(t)
\end{gathered}
$$

If no additional rescattering occurs, the backscattered electron leaves the laser field with an energy,

$$
E_{b s}=\frac{1}{2}\left[\mathbf{A}\left(t_{0}\right)-2 \mathbf{A}\left(t_{r}\right)\right]^{2} \in\left[0,10 U_{p}\right]
$$

Note the above derivation is valid in the "short pulse" regime, where the laser field turns off before the electron has a chance to leave the laser focus. Whereas in the "long pulse" regime, as the electron leaves the laser focus it experiences a force, $-\nabla U_{p}$, due to the laser inhomogeneity, therefore it gains an additional kinetic energy 
equal to $U_{p}[23,174]$. In this case we have,

$$
E_{d} \in\left[U_{p}, 3 U_{p}\right]
$$

The electron trajectories and $U_{p}$ scale of energy transfers can be nicely illustrated in the case of a linearly polarized driving laser with sinusoidal waveform $F=F_{0} \sin \omega t$, where the equation of motion can be easily solved,

$$
\begin{gathered}
\ddot{x}(t)=-F_{0} \sin \omega t, \quad x\left(t_{0}\right)=0, \dot{x}\left(t_{0}\right)=0 \\
\dot{x}(t)=\frac{F_{0}}{\omega}\left(\cos \omega t-\cos \omega t_{0}\right) \\
x(t)=\frac{F_{0}}{\omega^{2}}\left[\sin \omega t-\sin \omega t_{0}+\left(\omega t_{0}-\omega t\right) \cos \omega t_{0}\right]
\end{gathered}
$$

The results are shown in Fig. 1.9. 


\section{Appendix D}

\section{Femtosecond laser system}

The femtosecond laser system consists of a Ti:sapphire oscillator and a chirped pulse amplifier (CPA). A grating based stretcher/compressor is used to temporally stretch/re-compress the pulse before/after amplification. A grating based spectrometer is used for daily monitoring of the spectrum of the oscillator. A single-shot autocorrelator is used to measure the pulse duration after amplification.

Ti:sapphire Oscillator: The oscillator (KMLabs MTS Mini Ti:Sapphire Laser) is pumped by a frequency doubled $\mathrm{Nd}_{\mathrm{YVO}} \mathrm{CW}$ laser (Spectra-Physics Millennia Vs.) at $3.75 \mathrm{~W}$ (typically a pump power between $3.5 \mathrm{~W}$ and $4.5 \mathrm{~W}$ is used), and typical mode-locked output power is $300-500 \mathrm{~mW}$. Pulse energy, duration and repetition rate is $\sim 2 \mathrm{~nJ}, \sim 15-50$ fs and $\sim 90 \mathrm{MHz}$, respectively.

Grating spectrometer: The spectral components of the laser pulse from the oscillator are spatially stretched via diffraction on a grating and imaged by a CCD line camera whose output (representing the power spectrum) is displayed on an oscilloscope.

Grating stretcher: The stretcher is used to temporally expand the pulse by introducing a wavelength-dependent phase delay (linear chirp) in the pulse. This 
reduces the peak pulse intensity for the purpose of avoiding damage to optics and reducing non-linear effects (which increase with intensity) during amplification. The pulse is stretched from $\sim 30$ fs to $\sim 200$ ps by the stretcher.

Chirped pulse amplifier: The CPA consists of a regenerative (regen) preamplifier followed by a three-pass linear amplifier. Both amplifiers are pumped by a Q-switched frequency doubled Nd:YAG laser (Spectra-Physics Quanta Ray GCR) which produces pulses at a $15 \mathrm{~Hz}$ repetition rate with a pulse duration of $\sim 10 \mathrm{~ns}$ and a maximum average output power of $\sim 4 \mathrm{~W}$. The operation parameters [number of round-trips, pump power, output power, amplified spontaneous emission (ASE)] of the regen amplifier were originally $[16,580-620 \mathrm{~mW}, 60-65 \mathrm{~mW}, 5 \%]$ but later changed to $[20,(450-500) \mathrm{mW},(40-45) \mathrm{mW}, 5 \%]$ to avoid optical damage to the switch-in Pockels cell, which is located near the smallest beam waist in the regen cavity. The amplification achieved in the regen is on the order of $10^{6}$. The output power of the three-pass amplifier depends linearly on the pump power at sufficiently high pump energies (1.6-1.65 W pump results in 400-430 $\mathrm{mW}$ output, and $\sim 0.2 \mathrm{~W}$ increase in pump corresponds to $\sim 100 \mathrm{~mW}$ increase in output). The output is kept below $700 \mathrm{~mW}$ to avoid damage to optical elements.

Grating compressor: The compressor "undoes" the chirp introduced by the stretcher and re-constructs the ultrashort femtosecond pulse. Unfortunately it is not able to compensate for higher order dispersion introduced during amplification (mainly because of optical components inside the regen cavity) and the pulse is recompressed to $\sim 100-200$ fs. The $\sim 85 \%$ diffraction efficiency of the grating in the compressor results in a $\sim 50 \%$ loss in optical power.

Single-shot autocorrelator: The fundamental beam is split into two almost identical parts using two half-mirrors. The beams cross at an angle $2 \Phi$ in a frequency doubling crystal (BBO), inside which efficient SHG occurs when the two pulses are 
spatially and temporally overlapped. The intensity of the SHG varies along the spatial axis transverse to the second harmonic beam propagation. Different transverse locations $(x)$ in the SHG intensity distribution correspond to different relative delays $(\tau)$ between the two fundamental beams. Hence, the SHG intensity distribution as a function of the transverse spatial coordinate $(S(x))$ is proportional to the second order autocorrelation of the fundamental [175],

$$
\begin{gathered}
x=\frac{c \tau}{n \sin \Phi} \\
S(x)=\int_{-\infty}^{+\infty} I(t+\tau) I(t-\tau) \mathrm{d} t
\end{gathered}
$$

here $c$ is the speed of light in vacuum, $n$ is the index of refraction of the crystal and $I(t)$ is the intensity of the identical fundamental beams. By capturing the SHG intensity distribution with a CCD camera, we can characterize the fundamental pulse duration for each laser shot (provided that the angle $2 \Phi$ is sufficiently large that the fundamental beam diameter is much larger than the spatial extent of the autocorrelation signal).

To calibrate the autocorrelator, a thin glass slide with known thickness is put into either of the two fundamental beam paths to introduce a known temporal delay $(\Delta \tau)$ between the two pulses (see Fig. D.1), this causes a shift in x-position $(\Delta t)$ of the SHG signal monitored on the oscilloscope. The transverse FWHM of the SHG beam is $\tau_{F W H M s}=\Delta \tau /(2 \Delta t \sin \Phi) \times t_{F W H M s}$, here $t_{F W H M s}$ is the FWHM width of the second harmonic signal on the oscilloscope. Assuming a $\operatorname{sech}^{2}(t)$ shaped fundamental pulse, its FWHM is calculated as $\tau_{F W H M f}=\tau_{F W H M s} / 1.55$. In the experiments, the FWHM of the femtosecond pulses are typically measured to be $\sim 150$ fs at the output of the compressor. 


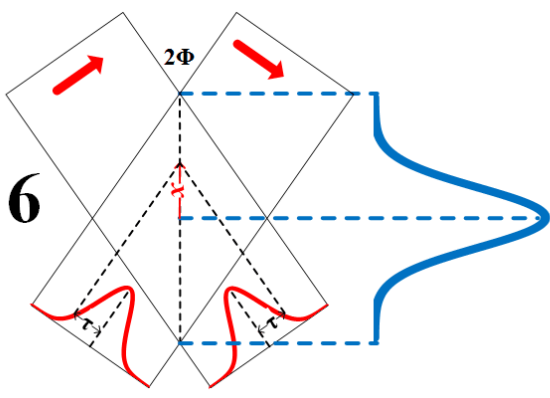

1. SHG beam ; 2 . fs beam $A$;

3. fs beam B; 4. BBO crystal;

5. $\Delta \tau$ delay slide;

6. illustration of temporal overlap

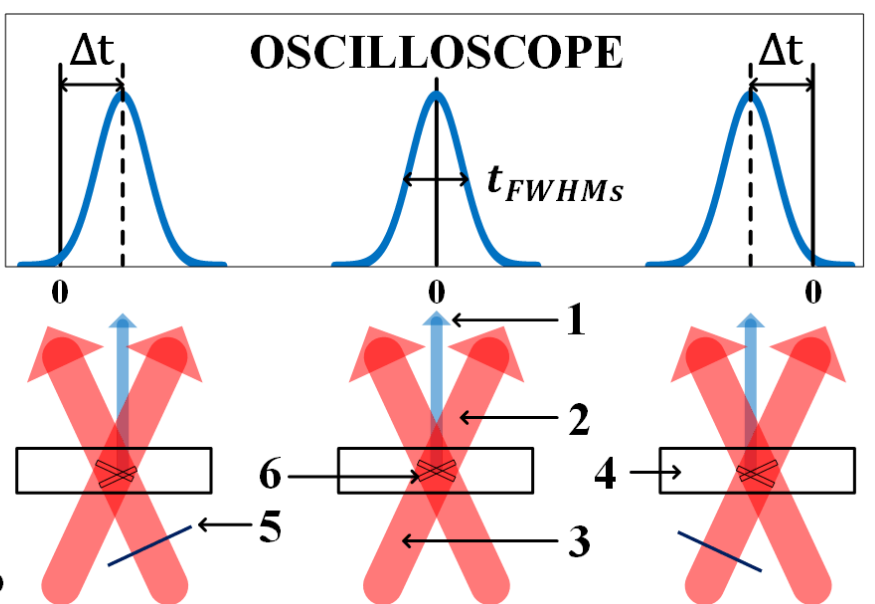

Figure D.1: Schematic of the single-shot autocorrelation and its operation.
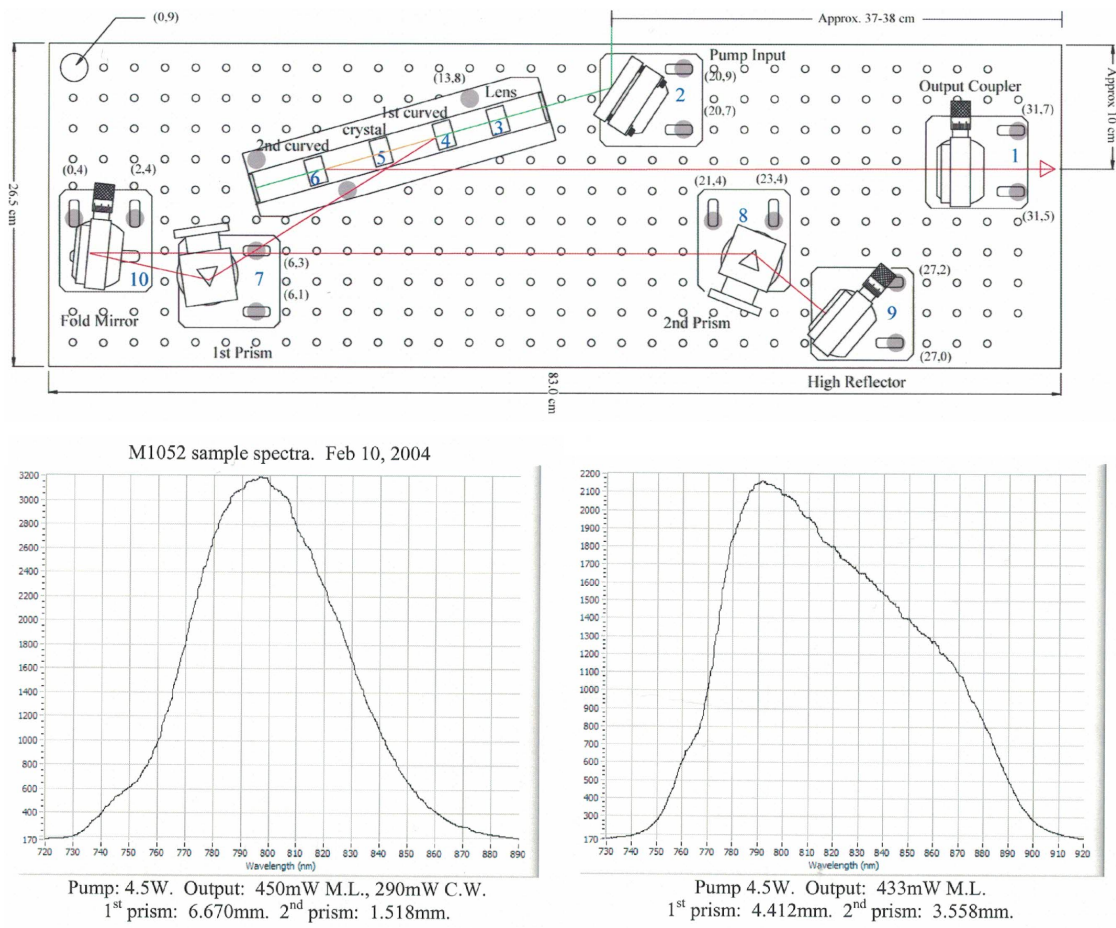

Figure D.2: Ti:sapphire oscillator and sample mode-locked output spectrum, from the manual of KMLabs MTS Mini Ti:Sapphire Laser. The mode lock is self-started due to the Kerr-lens effect (Kerr lens mode-locking). The prism pair is used to compensate for group velocity dispersion(GVD). The pump laser and the Ti:sapphire crystal in the oscillator are water-cooled to maintain a temperature of $\sim 65^{\circ} \mathrm{F}$. A photodiode picks up scattered light from the cavity and generates a signal for synchronizing the CPA pump laser and Pockels cells to the mode-locked pulse train. 


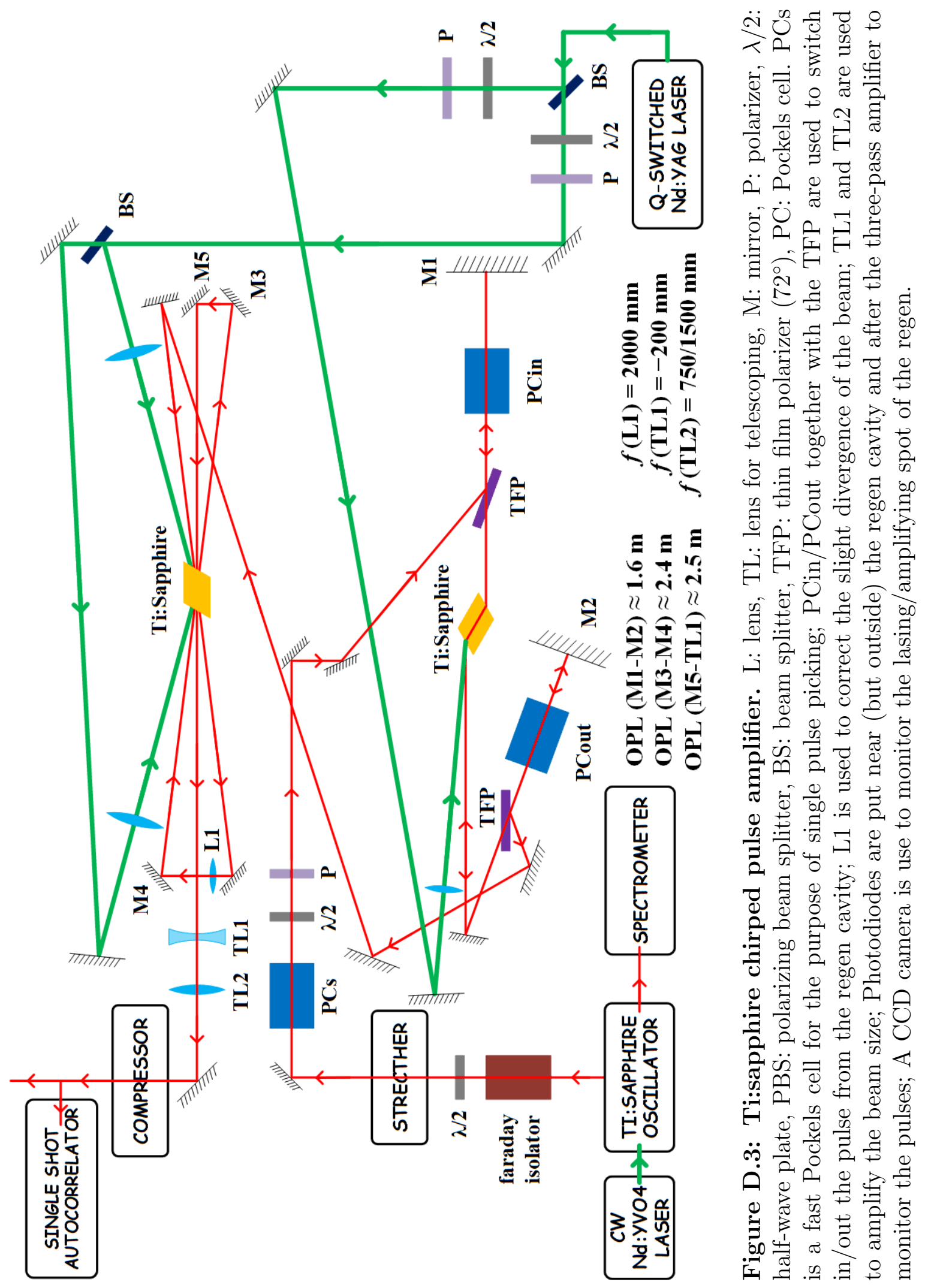




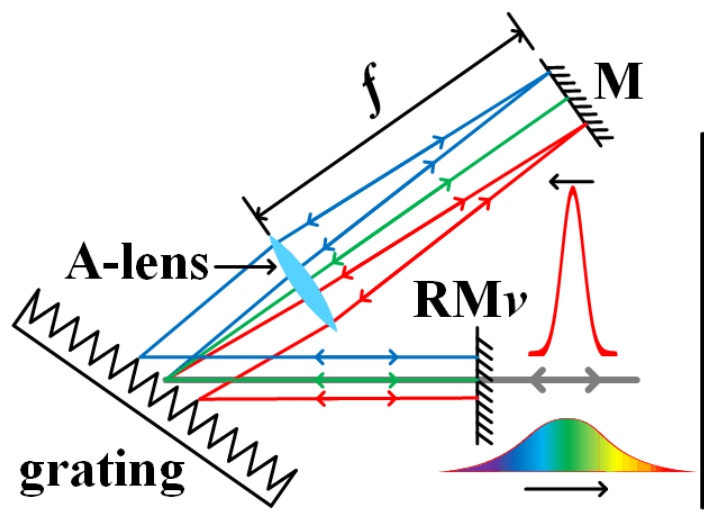

a grating stretcher

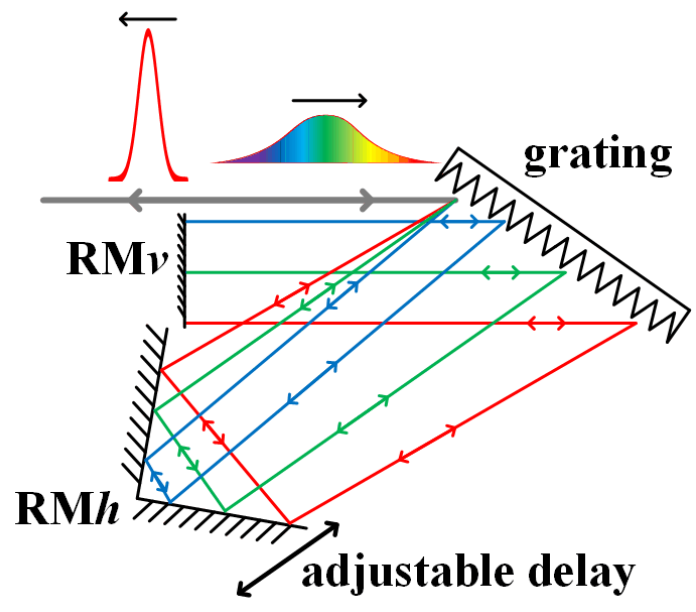

c grating compressor

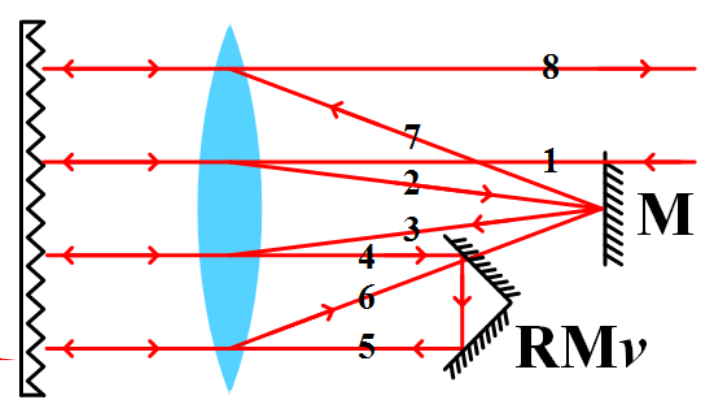

b stretcher sideview

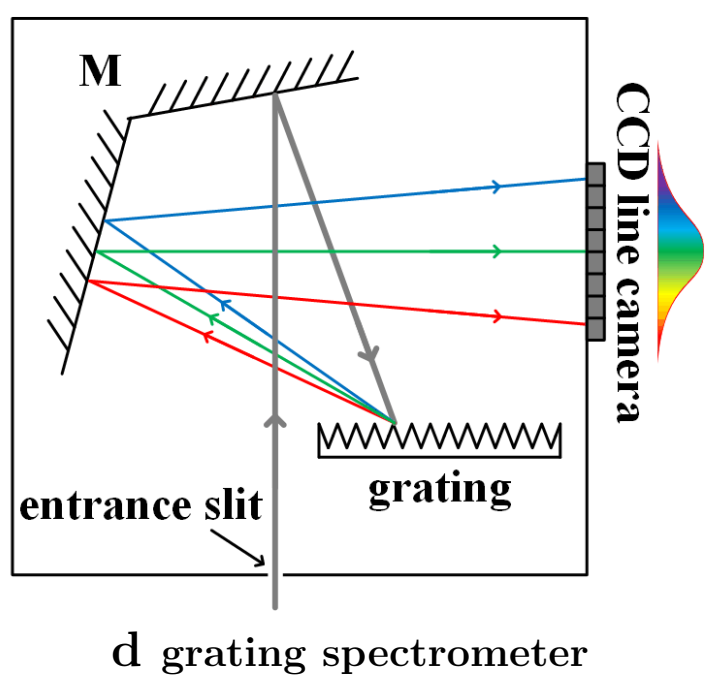

Figure D.4: Grating based pulse stretcher, compressor and spectrometer. M: mirror, A-lens: achromatic lens, RM $h$ : retroreflect mirrors that introduce a horizontal displacement, $\mathrm{RM} v$ : retroreflect mirror(s) that introduces a vertical displacement (this could be a single mirror which is slight tilted vertically or a pair of mirrors which are $90^{\circ}$ crossed). a) Grating stretcher: The grating is tilted at a angle of $54^{\circ}$ relative to the incident beam, the amount of positive GVD is controlled by the distance between the grating and the achromatic lens, a line-mask can be put in front of mirror $\mathrm{M}$ to narrow the spectrum, input and output beams are vertically separated by $\mathrm{RM} v$. b) Stretcher side-view: The beam hits the grating 4 times, and is horizontally stretched to a line (see a) and vertically focused on mirror M. c) Grating compressor: the grating is tilted at the same angle $\left(54^{\circ}\right)$ as the grating in stretcher, the amount of negative GVD is controlled by the distance between the grating and $\mathrm{RM} h$, input and output beams are vertically separated by RMv. d) Grating spectrometer: The entrance is covered by a neutral density filter to avoid saturation of the camera. The slit $(50 \mu \mathrm{m})$ narrows down the transverse beam size and ensures sufficient spectral resolution. 


\section{Appendix E}

\section{Electro-optic sampling}

The relationship between the electric field $\mathbf{E}$, polarization $\mathbf{P}$ and electric displacement $\mathbf{D}$ in a medium are given by,

$$
\begin{gathered}
\mathbf{P}=\varepsilon_{0} \chi \mathbf{E}=\varepsilon_{0}\left(\chi^{(1)}+\chi^{(2)} \mathbf{E}+\chi^{(3)} \mathbf{E}^{2}+\cdots\right) \mathbf{E} \\
\mathbf{D}=\varepsilon_{0} \mathbf{E}+\mathbf{P}=\varepsilon_{0} \varepsilon \mathbf{E} \\
\mathbf{E}=\varepsilon_{0}^{-1} \boldsymbol{\eta} \mathbf{D} \\
\chi=\chi^{(1)}+\chi^{(2)} \mathbf{E}+\chi^{(3)} \mathbf{E}^{2}+\cdots, \quad \varepsilon=\mathbf{I}_{3}+\boldsymbol{\chi}, \quad \boldsymbol{\eta}=\boldsymbol{\varepsilon}^{-1}
\end{gathered}
$$

Here $\boldsymbol{\chi}, \boldsymbol{\varepsilon}$ and $\boldsymbol{\eta}$ are the tensors of electric susceptibility, (relative) permittivity and (relative) impermeability, respectively. $\chi^{(i)}$ is the $i$ th order susceptibility tensor of rank $(i+1)$. Thermodynamics requires $\boldsymbol{\chi}, \boldsymbol{\varepsilon}$ and $\boldsymbol{\eta}$ to be symmetric, i.e., $\boldsymbol{\chi}=\boldsymbol{\chi}^{T}$, $\boldsymbol{\varepsilon}=\boldsymbol{\varepsilon}^{T}$ and $\boldsymbol{\eta}=\boldsymbol{\eta}^{T}$ (or $\chi_{i j}=\chi_{j i}, \varepsilon_{i j}=\varepsilon_{j i}$ and $\eta_{i j}=\eta_{j i}$ ).

The electrostatic energy density is given by,

$$
\omega=\frac{1}{2} \mathbf{E} \cdot \mathbf{D}=\frac{1}{2 \varepsilon_{0}} \mathbf{D}^{T} \boldsymbol{\eta} \mathbf{D}=\frac{1}{2 \varepsilon_{0}} \sum_{i, j} \eta_{i j} D_{i} D_{j}
$$

Eq. E.2 gives a constant energy density surface in the $\mathbf{D}$ space, and if we define a 
dimensionless vector along the direction of $\mathbf{D}$ by $\boldsymbol{u}=\mathbf{D} / \sqrt{2 \varepsilon_{0} \omega}$, we obtain,

$$
\boldsymbol{u}^{T} \boldsymbol{\eta} \boldsymbol{u}=\sum_{i, j} \eta_{i j} u_{i} u_{j}=1
$$

In the principal coordinates, defined as the orthogonal coordinate system where $\chi / \varepsilon / \boldsymbol{\eta}$ are diagonal matrices, Eq. E.3 becomes,

$$
\eta_{1} u_{1}^{2}+\eta_{2} u_{2}^{2}+\eta_{3} u_{3}^{2}=1
$$

Introducing the main refractive indices,

$$
n_{i}=\sqrt{\varepsilon_{i}}=\sqrt{1 / \eta_{i}}
$$

Eq. E.4 becomes the well-known index ellipsoid equation,

$$
\frac{u_{1}^{2}}{n_{1}^{2}}+\frac{u_{2}^{2}}{n_{2}^{2}}+\frac{u_{3}^{2}}{n_{3}^{2}}=1
$$

In crystal optics, the index ellipsoid provides a helpful way of finding the optical axes of a crystal, as well as the refractive indices and polarization axes for a given electric wave vector.

The impermeability tensor $\boldsymbol{\eta}$ can be expanded as a function of the applied electric field in the following form,

$$
\begin{aligned}
\eta_{i j}(\mathbf{E}) & =\eta_{i j}(0)+\left.\sum_{k} \frac{\partial \eta_{i j}}{\partial E_{k}}\right|_{E=0} E_{k}+\left.\sum_{k, l} \frac{1}{2} \frac{\partial^{2} \eta_{i j}}{\partial E_{k} \partial E_{l}}\right|_{E=0} E_{k} E_{l}+\cdots \\
& =\eta_{i j}(0)+\sum_{k} r_{i j k} E_{k}+\sum_{k, l} s_{i j k l} E_{k} E_{l}+\cdots
\end{aligned}
$$

Here $r_{i j k}=r_{j i k}=\left.\frac{\partial \eta_{i j}}{\partial E_{k}}\right|_{E=0}$ and $s_{i j k l}=s_{j i k l}=s_{i j l k}=s_{j i l k}=\left.\frac{1}{2} \frac{\partial^{2} \eta_{i j}}{\partial E_{k} \partial E_{l}}\right|_{E=0}$ are the Pockels and Kerr coefficients respectively. From the previous discussion, we know one can determine the index ellipsoid of a crystal by performing the principal axis 
transformation (orthogonal diagonalization of $\boldsymbol{\eta}$ ),

$$
\mathbf{U}^{-1} \boldsymbol{\eta} \mathbf{U}=\left(\begin{array}{ccc}
\frac{1}{n_{1}{ }^{2}} & 0 & 0 \\
0 & \frac{1}{n_{2}{ }^{2}} & 0 \\
0 & 0 & \frac{1}{n_{3}^{2}}
\end{array}\right)
$$

Zinc telluride ( $\mathrm{ZnTe})$ falls in the space group of zincblende structure $(\mathrm{F} \overline{4} 3 \mathrm{~m})$ and has a high degree of symmetry. It is the most commonly used electro-optic crystal for EOS. It is optically isotropic at vanishing electric fields, i.e., $n_{1}(0)=$ $n_{2}(0)=n_{3}(0)=n_{0}$, and the tensor $\boldsymbol{r}$ contains only one independent entry $r_{41}=$ $r_{52}=r_{63}$ (Note elements of $\boldsymbol{r}$ are redefined according to symmetry $r_{i j k}=r_{j i k}$ : $\left.r_{11 k / 22 k / 33 k / 23 k=32 k / 13 k=31 k / 12 k=21 k} \triangleq r_{1 k / 2 k / 3 k / 4 k / 5 k / 6 k}, k=1,2,3\right)$.

In the experiment, a (110) cut ZeTe crystal is used, and the THz and NIR pulses are incident perpendicular to the (110) plane. For clarity, in the following derivation, two coordinate systems are used: the base system $(\hat{\boldsymbol{x}}, \hat{\boldsymbol{y}}, \hat{\boldsymbol{z}})$ of the cubic crystal lattice, where $\hat{\boldsymbol{x}}=(1,0,0)^{T}, \hat{\boldsymbol{y}}=(0,1,0)^{T}$, and $\hat{\boldsymbol{z}}=(0,0,1)^{T}$, and a redefined system $(\hat{\mathbf{X}}, \hat{\mathbf{Y}}, \hat{\mathbf{Z}})$ according to the crystal cut, where $\hat{\mathbf{X}}=\frac{1}{\sqrt{2}}(1,-1,0)^{T}, \hat{\mathbf{Y}}=(0,0,1)^{T}$, and $\hat{\mathbf{Z}}=\frac{1}{\sqrt{2}}(-1,-1,0)^{T}$. The THz pulse propagates along $\hat{\mathbf{Z}}$ and is linearly polarized with an angle $\alpha$ relative to $\hat{\mathbf{X}}$.

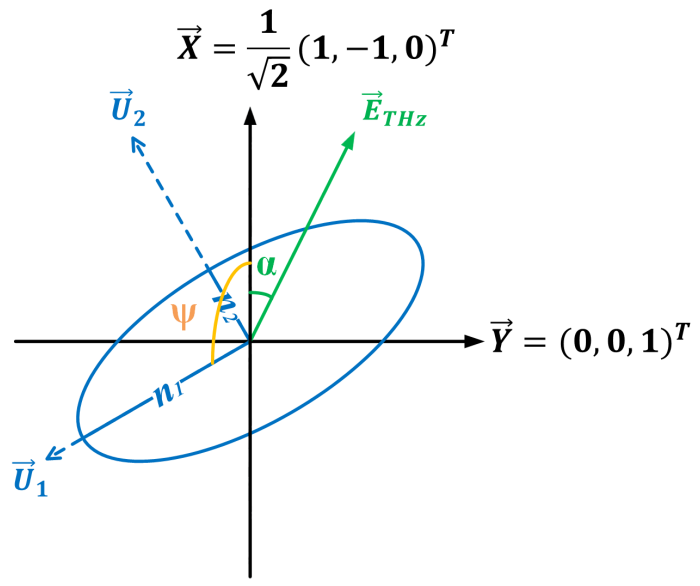

Figure E.1: The THz field modified the index ellipse in the (110) plane. 
In the $(\hat{\boldsymbol{x}}, \hat{\boldsymbol{y}}, \hat{\boldsymbol{z}})$ basis, $\mathbf{E}_{T H z}=E_{T H z}\left(\frac{1}{\sqrt{2}} \cos \alpha,-\frac{1}{\sqrt{2}} \cos \alpha, \sin \alpha\right)^{T}$, and the impermeability tensor $\boldsymbol{\eta}$ is modified to,

$$
\boldsymbol{\eta}=\frac{1}{n_{0}^{2}}\left(\begin{array}{ccc}
1 & 0 & 0 \\
0 & 1 & 0 \\
0 & 0 & 1
\end{array}\right)+r_{41} E_{T H z}\left(\begin{array}{ccc}
0 & \sin \alpha & -\frac{1}{\sqrt{2}} \cos \alpha \\
\sin \alpha & 0 & \frac{1}{\sqrt{2}} \cos \alpha \\
-\frac{1}{\sqrt{2}} \cos \alpha & \frac{1}{\sqrt{2}} \cos \alpha & 0
\end{array}\right)
$$

The eigenvalues and eigenvectors of $\boldsymbol{\eta}$ are:

$$
\begin{gathered}
\lambda_{1,2}=\frac{1}{n_{0}{ }^{2}}-\frac{r_{41} E_{T H z}}{2}\left(\sin \alpha \pm \sqrt{1+3 \cos ^{2} \alpha}\right), \lambda_{3}=\frac{1}{n_{0}^{2}}+r_{41} E_{T H z} \sin \alpha \\
\hat{\mathbf{U}}_{1}=\frac{1}{2} \sqrt{1+\frac{\sin \alpha}{\sqrt{1+3 \cos ^{2} \alpha}}\left(-1,1,-\frac{2 \sqrt{2} \cos \alpha}{\sqrt{1+3 \cos ^{2} \alpha}+\sin \alpha}\right)^{T}} \\
\hat{\mathbf{U}}_{2}= \begin{cases}\frac{1}{2} \sqrt{1-\frac{\sin \alpha}{\sqrt{1+3 \cos ^{2} \alpha}}}\left(1,-1,-\frac{2 \sqrt{2} \cos \alpha}{\sqrt{1+3 \cos ^{2} \alpha}-\sin \alpha}\right)^{T} & \alpha \neq \pi / 2 \\
(0,0,1)^{T}=\hat{\mathbf{Y}} & \alpha=\pi / 2 \\
\hat{\mathbf{U}}_{3}=\frac{1}{\sqrt{2}}(-1,-1,0)^{T}=\hat{\mathbf{Z}} & \end{cases}
\end{gathered}
$$

Considering that $r_{41} E_{T H z} \ll 1 / n_{0}^{2}$, the main refractive indices $n_{i}=1 / \sqrt{\lambda_{i}}$ can be approximated as,

$$
\begin{aligned}
& n_{1}=n_{0}+\frac{n_{0}{ }^{3} r_{41} E_{T H z}}{4}\left(\sin \alpha+\sqrt{1+3 \cos ^{2} \alpha}\right) \rightarrow n_{s} \\
& n_{2}=n_{0}+\frac{n_{0}{ }^{3} r_{41} E_{T H z}}{4}\left(\sin \alpha-\sqrt{1+3 \cos ^{2} \alpha}\right) \rightarrow n_{f} \\
& n_{3}=n_{0}-\frac{n_{0}{ }^{3} r_{41} E_{T H z}}{2} \sin \alpha
\end{aligned}
$$

The directions of the eigenvectors are the directions of the principal axes. Clearly $\hat{\mathbf{U}}_{3}$ is perpendicular to the (110) plane whereas $\hat{\mathbf{U}}_{1}$ and $\hat{\mathbf{U}}_{2}$ lie in the (110) plane. The angle $\psi$ between $\hat{\mathbf{U}}_{1}$ and $\hat{\mathbf{X}}$ is linked to $\alpha$ by,

$$
\cos 2 \psi=\frac{\sin \alpha}{\sqrt{1+3 \cos ^{2} \alpha}}
$$


From Eq. E.12, by applying the THz pulse, a change of refractive indices $\Delta n_{i}$, which is proportional to the electric field $E_{T H z}$, is induced. If the NIR pulse is incident along $\hat{\mathbf{Z}}$, a phase shift will therefore be introduced to the two polarization components (along $\hat{\mathbf{U}}_{1}$ and $\hat{\mathbf{U}}_{2}$ ) of its electric vector $\mathbf{E}_{N I R}$,

$$
\Gamma(\alpha)=\frac{\omega d}{c}\left(n_{s}-n_{f}\right)=\frac{\omega d}{2 c} n_{0}^{3} r_{41} E_{T H z} \sqrt{1+3 \cos ^{2} \alpha} \propto E_{T H z}
$$

The phase shift is proportional to $E_{T H z}$, and reaches maximum at $\alpha=0$. By "measuring" the phase shift, one can then determine the THz field strength.

The setup to "measure" the phase shift consists of the ZnTe crystal, a $45^{\circ}$ rotated quarter wave plate (QWP), a wollaston prism (WP) (or polarizing beam splitter (PBS)) and a balanced diode detector. Using the Jones calculus, and set $\boldsymbol{R}(\varphi), \boldsymbol{Q}$, and $\boldsymbol{Z T}$ to be the matrices for the rotation operator, QWP, and ZeTe phase retarder, respectively,

$$
\begin{aligned}
\boldsymbol{R}(\varphi) & =\left(\begin{array}{cc}
\cos \varphi & \sin \varphi \\
-\sin \varphi & \cos \varphi
\end{array}\right) \\
\boldsymbol{Q} & =\left(\begin{array}{cc}
e^{-i \pi / 4} & 0 \\
0 & e^{i \pi / 4}
\end{array}\right) \\
\boldsymbol{Z T}=\left(\begin{array}{cc}
e^{-i n_{s} \omega d / c} & 0 \\
0 & e^{-i n_{f} \omega d / c}
\end{array}\right) & =e^{-i \phi}\left(\begin{array}{cc}
e^{-i \Gamma(\alpha) / 2} & 0 \\
0 & e^{i \Gamma(\alpha) / 2}
\end{array}\right), \phi=\frac{\left(n_{s}+n_{f}\right) \omega d}{2 c}
\end{aligned}
$$

In the $(\hat{\mathbf{X}}, \hat{\mathbf{Y}}, \hat{\mathbf{Z}})$ basis, the fast axis of the ZnTe and the QWP is rotated by $\psi$ and $45^{\circ}$ relative to the horizontal $\hat{\mathbf{X}}$, respectively. The electric vector of the NIR pulse 
after the QWP can then be calculated by,

$$
\begin{gathered}
\mathbf{E}_{N I R}^{\prime}=\boldsymbol{R}(-\pi / 4) \boldsymbol{Q R}(\pi / 4) \boldsymbol{R}(-\psi) \boldsymbol{Z} \boldsymbol{T} \boldsymbol{R}(\psi) \mathbf{E}_{N I R}=\left(E_{N I R \|}^{\prime}, E_{N I R \perp}^{\prime}\right)^{T} \\
E_{N I R \|}^{\prime}=(1,0) \mathbf{E}_{N I R}^{\prime}, \quad E_{N I R \perp}^{\prime}=(0,1) \mathbf{E}_{N I R}^{\prime}
\end{gathered}
$$

The WP (or PBS) splits the horizontal and vertical polarization components, which are then guided to and detected by the balanced diode detector. The final signal is given by,

$$
S \propto E_{N I R \|}^{\prime 2}-E_{N I R \perp}^{\prime 2}
$$

When the incident NIR probe pulse is horizontally polarized, i.e., $\mathbf{E}_{N I R}=E_{N I R}(1,0)^{T}$, by performing the Jones calculus, the signal of the balanced diode detector is,

$$
S \propto E_{N I R}^{2} \sin (\Gamma(\alpha)) \propto \sin (\Gamma(\alpha))
$$

Recall that $\Gamma(\alpha)$ is maximum at $\alpha=0$, therefore the best sensitivity of the detector is reached when the incident $\mathrm{THz}$ pulse is also horizontally polarized. 


\section{Appendix F}

\section{Time-of-flight spectrometer and retarding potential energy analyzer}




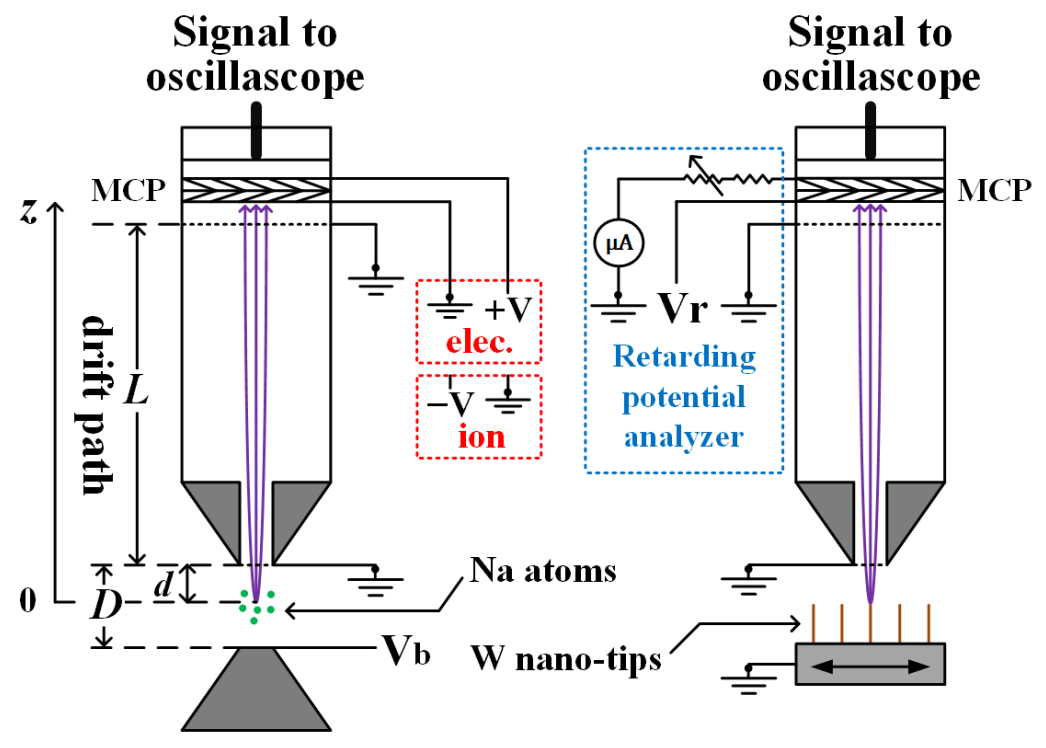

Figure F.1: Left) Time of flight electron spectrometer and Right) retarding potential energy analyzer.

Fig. F.1Left is a schematic of the time-of flight spectrometer. The calibration was carried out by recording the TOF of $\sim 100 \mathrm{meV}$ femtosecond photoelectron bursts (produced via single-photon ionization from the $\mathrm{Na} 3 p_{1 / 2}$ state to the continuum by $150 \mathrm{fs}, 395 \mathrm{~nm}$ frequency-doubled Ti:Sapphire laser pulses) under various bias potentials. We have,

$$
\begin{aligned}
\text { TOF } & =t-t_{0} \\
& =\frac{m_{e} D}{e V_{b}}\left[\left(v_{0}^{2}+\frac{2 d}{D} \frac{e V_{b}}{m_{e}}\right)^{1 / 2}-v_{0}\right]+L\left(v_{0}^{2}+\frac{2 d}{D} \frac{e V_{b}}{m_{e}}\right)^{-1 / 2} \\
& =\frac{d+L}{v_{0}}, \quad V_{b}=0, v_{0}>0 \\
& =\sqrt{\frac{m_{e}}{e}}\left(\sqrt{\frac{2 d}{D}} D+\sqrt{\frac{D}{2 d}} L\right) V_{b}^{-1 / 2}, \quad v_{0}=0, \quad V_{b}>0
\end{aligned}
$$

Here $m_{e}$ is the electron mass, $e$ is the elementary charge, $t_{0}$ is the trigger time of the oscilloscope, $v_{0}$ is the initial velocity of the photoelectrons, $V_{b}$ is the bias voltage, $L$ is the field free drift path, $d$ is the field dependent acceleration path, and $D$ is the separation between the two plates of the interaction region. 
The entrance aperture of the detector tube is about $1 \mathrm{~cm}$ in diameter and $3 \mathrm{~cm}$ in height, TOF calibration gives $L=13.6 \mathrm{~cm}, d=2 \mathrm{~cm}$ and $d_{1}=1 \mathrm{~cm}$, detecting acceptance angle is about $\pm 2^{\circ}$ in this configuration.

a

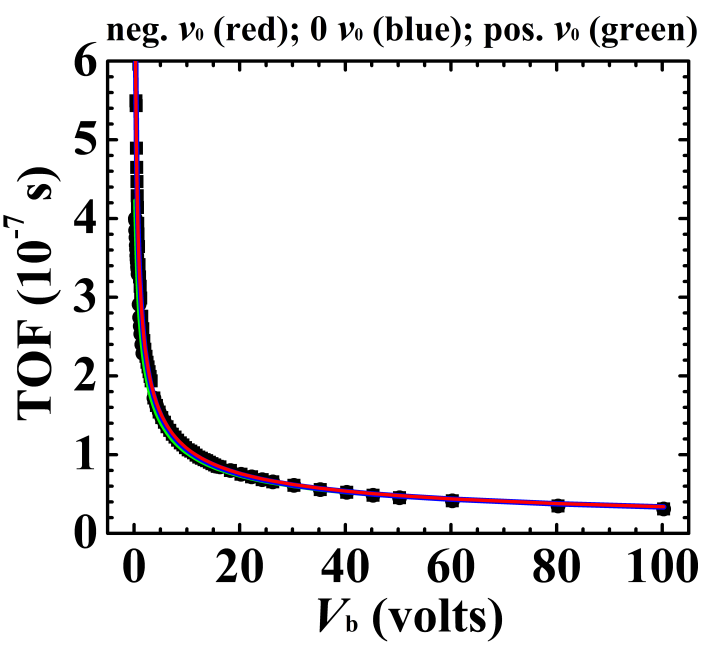

C

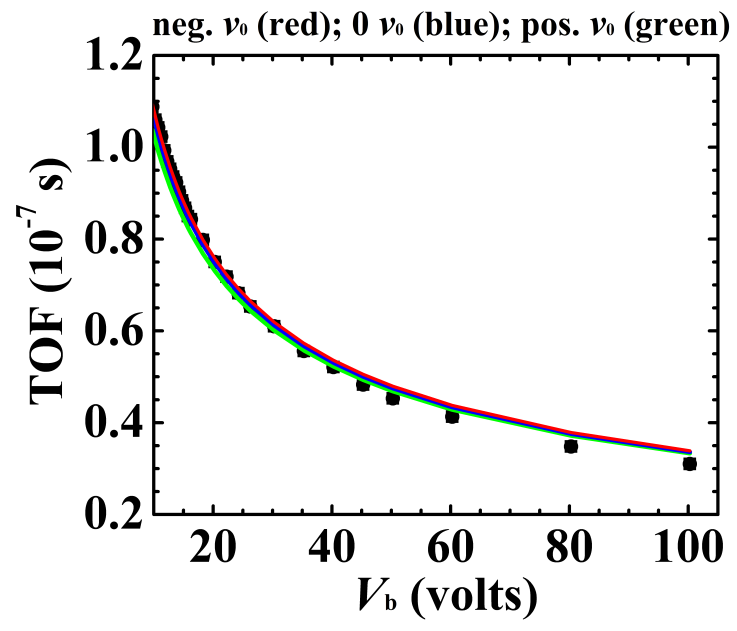

b

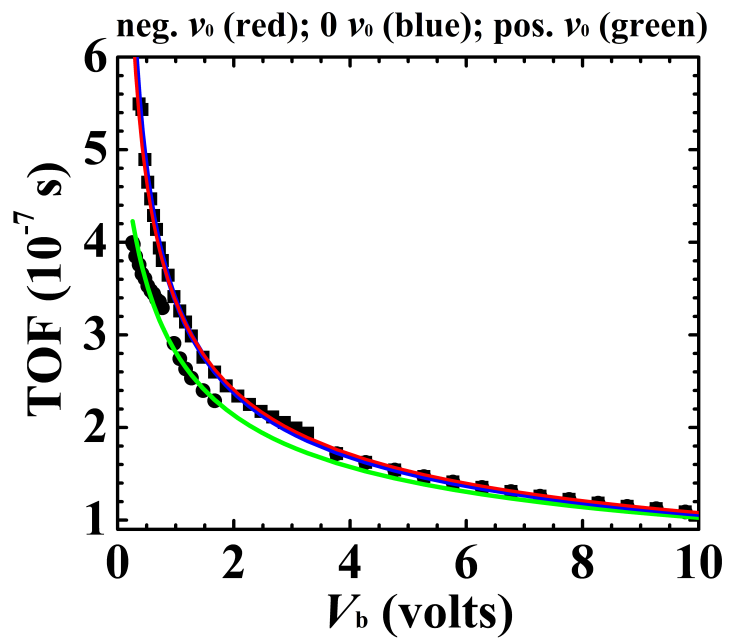

d

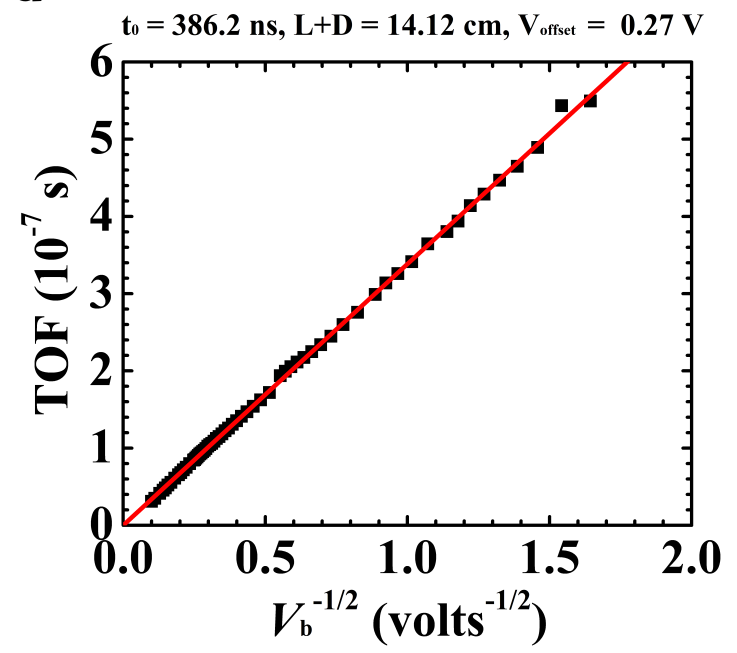

Figure F.2: TOF electron spectrometer calibration results. At low bias voltage, there are two delay peaks of electron signal (see Fig. b), due to up (postive $v_{0}$ ) or down (negative $v_{0}$ ) electron emissions. The two signals merge at high bias voltage. Figs. a-c show the time of flight calibration. It was found that the late delay peak is equally well described by $v_{0}=0 \mathrm{~m} / \mathrm{s}$ or $v_{0}=-2.23 \times 10^{5} \mathrm{~m} / \mathrm{s}$. So assuming $v_{0}=0 \mathrm{~m} / \mathrm{s}$, it is straightforward to find the best fit values by plotting the TOF as a function of $V_{b}^{-1 / 2}$ (Fig. d). 

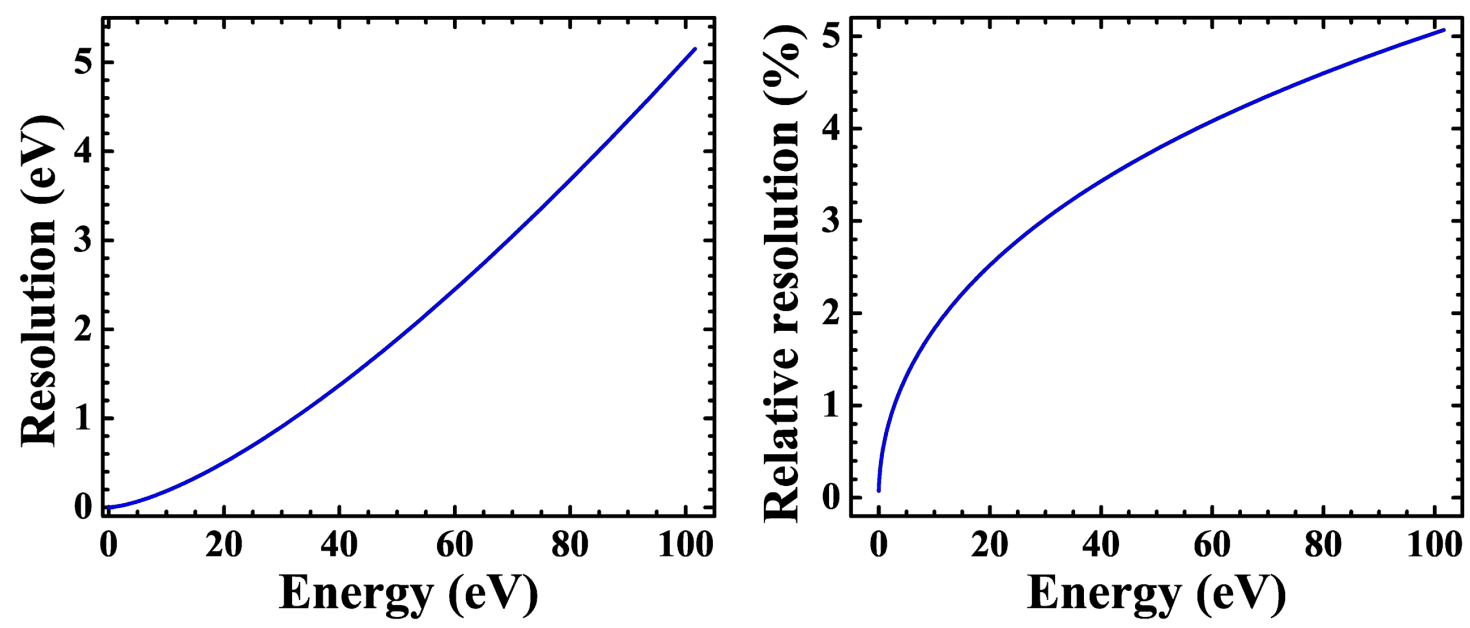

Figure F.3: Resolution of the LeCroy 9350A oscilloscope for time-of-flight measurement of electron energy.

The design of the RPA is shown in Fig. F.1Right: The retarding voltage $V_{r}$ $\left(V_{r} \leq 0\right)$ is directly added to the entrance side of the MCPs and an external voltage divider circuit is used to keep the voltage across the MCPs $\left(V_{m c p}\right)$ constant. During the measurements, when changing $V_{r}$, if $-V_{r} \leq V_{m c p}$, the exit side of the MCPs is positively biased with voltage $V=V_{m c p}+V_{r}$, whereas if $-V_{r}>V_{m c p}$, it is grounded and $V_{m c p}$ is kept constant by adjusting a potentiometer so that the reading of the micro-current meter remains unchanged.

In the experiments, $V_{m c p}$ is hold fixed at a value between $1400 \mathrm{~V}$ and $2000 \mathrm{~V}$ such that the electron signal on the oscilloscope is sufficiently big but does not exceed 200 $\mathrm{mV}$. In this case the micro-current meter has a reading of several tens of $\mu A$ and its resolution is $0.1 \mu \mathrm{A}$. This yields an energy resolution of several eV (e.g. For $V_{m c p}$ $=1.5 / 1.8 \mathrm{kV}, I \simeq 66 / 87 \mu A, \Delta I=0.1 \mu A$ corresponds to $\Delta E=2.3 / 2.1 \mathrm{eV})$. The energy resolution also depends on the design of the detector, mainly on how well it collimates the electron beam (e.g. A $2^{\circ} / 10^{\circ}$ divergence corresponds to a $0.6 \% / 1.5 \%$ relative error). Our detector geometry confines the detection angle within $\pm 2^{\circ}$. For 
the $\mathrm{THz}$ induced field emission experiments, the electrons are found to have very high energies $(\mathrm{keV})$ with spectra bandwidth hundreds of $\mathrm{eV}$, In this case, errors due to the finite resolution of the micro-current meter or the divergence cause by detector design are negligible. Accordingly divergence due to space charge in the electron burst is main source of spectral broadening.

It is worth noting that a typical RPA uses extra grids to apply the retarding voltage, the reason it was done differently here was for convenience. The detector was originally constructed without RPA grids, and significant modification would haven been required to add high voltage grids that did not induce arcing. Instead, by adding an external circuit, no modification to the detector were required to allow it to function as an RPA. The disadvantage was that due to the required potentiometer adjustment, data collection time was considerably longer. 


\section{Appendix G}

\section{Circuit diagram of the fast switch for electrochemical etching}

Fig. G.1 is the circuit diagram of the fast switch for electrochemical etching.

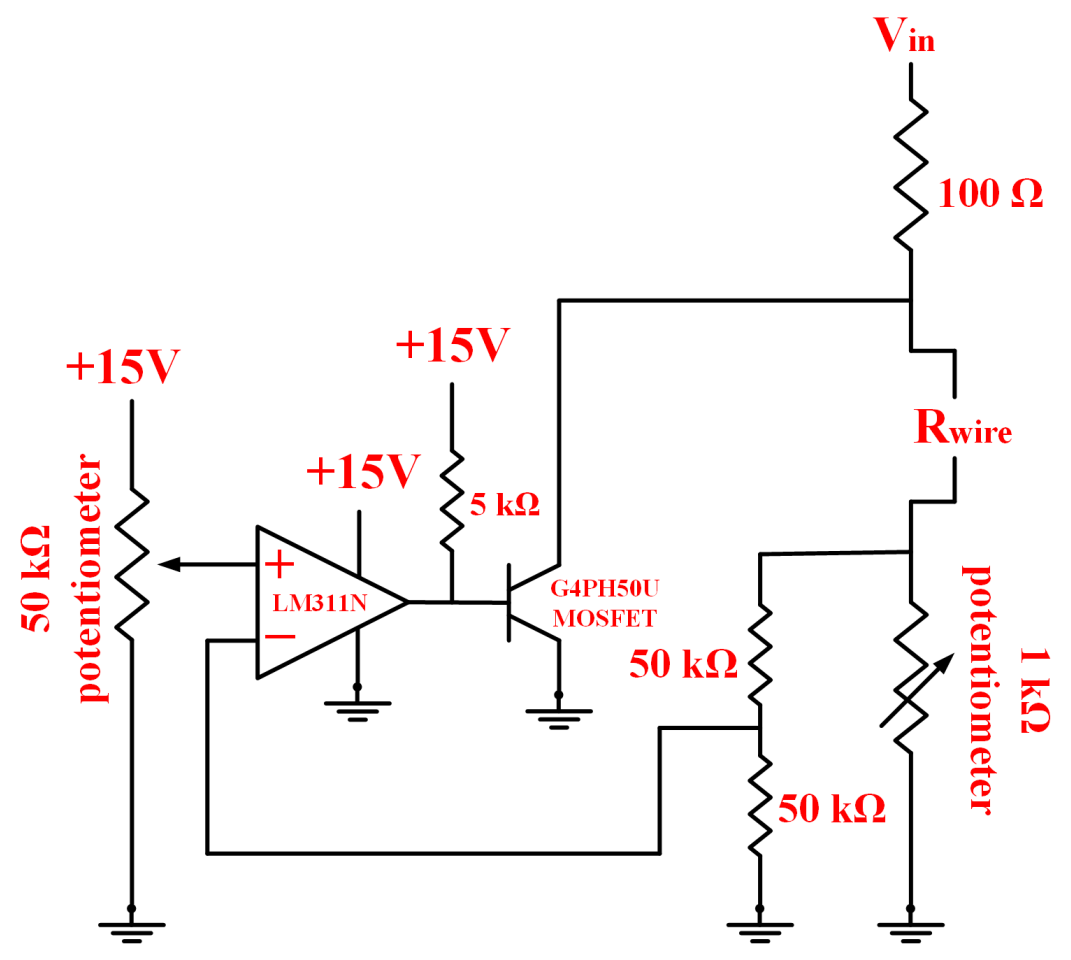

Figure G.1: Circuit diagram of the fast switch for electrochemical etching. 


\section{Appendix $\mathbf{H}$}

\section{Landau-Zener transition}

Landau-Zener transitions $[128,129]$ describe the transfer of population at or near avoided-crossings among energy levels. The simplest case is a two-level system whose Hamiltonian depends on some variable $q$, and the two energy levels cross at some value $q=q_{0}$ (Fig. H.1 dashed red lines). If the Hamiltonian is perturbed by an interaction which couples the two levels, the degeneracy of the system is broken, and the crossing becomes an avoided-crossing (Fig. H.1 solid blue lines) [130].

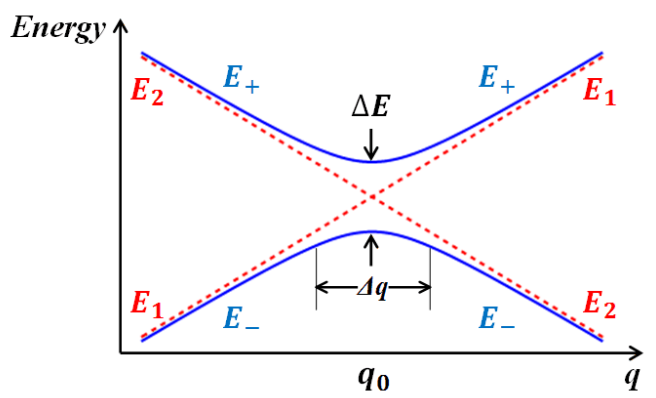

Figure H.1: Two-level system with an avoided energy crossing $\Delta E$.

If the system is initially prepared in state $|+\rangle$ at time $t=-\infty$, and $q$ increases in time, sweeping the energies through the avoided-crossing, in which state(s) will the population reside at $t=\infty$ ? For the simplest case, where the unperturbed energies 
$E_{1,2}$ vary linearly with $q$, and $q$ changes linearly with $t$, the problem is analytically solvable and the diabatic transition probability, i.e., the probability of finding the system in state $|-\rangle$, is given by the Landau-Zener formula [128-130],

$$
\begin{gathered}
P=e^{-2 \pi \Gamma} \\
\Gamma=\frac{(\Delta E / 2)^{2}}{\left|\frac{\mathrm{d} E_{1}}{\mathrm{~d} t}-\frac{\mathrm{d} E_{2}}{\mathrm{~d} t}\right|}=\frac{(\Delta E / 2)^{2}}{\left|\dot{q}\left(\frac{\mathrm{d} E_{1}}{\mathrm{~d} q}-\frac{\mathrm{d} E_{2}}{\mathrm{~d} q}\right)\right|}
\end{gathered}
$$

Apparently the behavior at the avoided-crossing depends on the slope of the energy levels and the rate at which $q$ changes with time (the slew rate), compared to the size of the avoid-crossing. At known $\Delta E$ and $\frac{\mathrm{d}\left(E_{1}-E_{2}\right)}{\mathrm{d} q}$, a critical slew rate of $q$ can be defined [10],

$$
S_{c}=\frac{(\Delta E)^{2}}{\frac{\mathrm{d} E_{1}}{\mathrm{~d} q}-\frac{\mathrm{d} E_{2}}{\mathrm{~d} q}}
$$

such that if $\dot{q} \gg S_{c}, P \rightarrow 1$ and the avoided crossing is traversed diabatically, whereas if $\dot{q} \ll S_{c}, P \rightarrow 0$ and the avoided crossing is traversed adiabatically. For an intermediate slew rate, the population is coherently split with some amplitude in $|+\rangle$ and some in $|-\rangle$.

If we define the width of the avoided crossing,

$$
\Delta q=\frac{\Delta E}{\left|\frac{\mathrm{d} E_{1}}{\mathrm{~d} q}-\frac{\mathrm{d} E_{2}}{\mathrm{~d} q}\right|_{q_{0}}}
$$

$\dot{q} \gg S_{c}$ indicates that the avoided crossing is traversed rapidly compared to $1 / \Delta E$, whereas if $\dot{q} \ll S_{c}$, the avoided crossing is traversed in a time much longer than $1 / \Delta E$.

Note in the limit of $\Delta E=0, P=1$ and the avoided crossing is traversed diabatically regardless of the slew rate. 


\section{Appendix I}

\section{Calibration of the unsaturated ionization probability}

In the experiments, ground state $\left(3 s_{1 / 2}\right) \mathrm{Na}$ atoms are dye laser excited through the $3 p_{1 / 2}$ state to the $n d$ Rydberg state. The atoms are then exposed to and ionized by the intense single-cycle $\mathrm{THz}$ pulses. The ionization yield signal detected by the micro-channel plate detector is,

$$
S_{n} \propto P_{3 s-3 p} P_{3 p-n d} P_{n} N_{3 s}
$$

here $P_{3 s-3 p}$ and $P_{3 p-n d}$ are the $3 s \rightarrow 3 p$ and $3 p \rightarrow n d$ excitation probability, respectively. $P_{n}$ is the THz ionization probability for the $n d$ state. $N_{3 s}$ is the number of atoms in the ground state. In the experiments, $P_{3 s-3 p}$ and $N_{3 s}$ do not change with $n$. Therefore, Eq. I.1 can be simplified to,

$$
S_{n} \propto P_{3 p-n d} P_{n}
$$

The $3 p \rightarrow n d$ excitation probability, below saturation, is proportional to both the excitation crossing section $\sigma_{n} \propto 1 / n^{3}$ and the energy $E_{n}$ of the dye laser that drives 
the excitation,

$$
P_{3 p-n d} \propto \sigma_{n} E_{n} \propto \frac{E_{n}}{n^{3}}
$$

Therefore we have,

$$
\frac{S_{n}}{P_{n}} \propto \frac{E_{n}}{n^{3}} \quad \text { or } \quad P_{n} \propto \frac{n^{3} S_{n}}{E_{n}}
$$

At known excitation dye laser intensity and fixed $\mathrm{THz}$ intensity, by comparing the ionization yield signal of a state $n_{a}$ whose ionization is below saturation $P_{n_{a}}<1$ with that of a state $n_{b}$ whose ionization is saturated $P_{n_{b}}=1$, we can find out the ionization probability of state $n_{a}$,

$$
P_{n_{a}}=\frac{n_{a}^{3} S_{n_{a}} E_{n_{b}}}{n_{b}^{3} S_{n_{b}} E_{n_{a}}} P_{n_{b}}=\frac{n_{a}^{3} S_{n_{a}} E_{n_{b}}}{n_{b}^{3} S_{n_{b}} E_{n_{a}}}
$$

Alternatively, to increase accuracy, in the experiments, we fix the $\mathrm{THz}$ intensity and vary the excitation dye laser intensity $E_{n}$ and record the ionization yield signal $S_{n}$ as $E_{n}$ increases for states $n_{a}$ and $n_{b}$. For each state, we find out a factor $A_{n}$ such that the plot of $S_{n} / A_{n}$ as a function of $E_{n} / n^{3}$ follow the same scaling. From Eq. I.4, clearly $P_{n} \propto A_{n}$ and the ionization probability of the state whose ionization is below saturation can be calibrated.

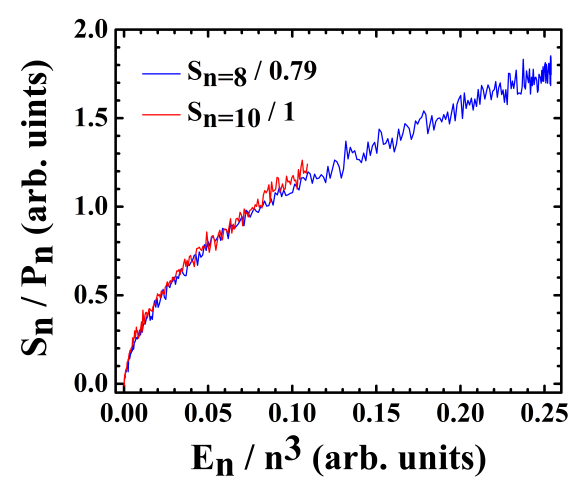

Figure I.1: Calibration of the ionization probability for a state whose ionization is below saturation. At the maximum applied $\mathrm{THz}$ field $F_{m} \simeq 430$ $\mathrm{kV} / \mathrm{cm}$, the ionization probability of the $n=10$ state is saturated and the ionization probability of the $n=8$ state is about 0.79 . 


\section{Appendix $\mathbf{J}$}

\section{Simulation of the field-emission}

\section{electron energy spectra}

The electron energy spectra are simulated with an adapted two-step model [140]. The first step is the field induced tunneling electron emission, from which the electron current density can be calculated. The second step is the subsequent field assisted electron acceleration, from which the electron energy can be found.

Approximate analytic forms for both the time-dependent tunneling probability and the spatial distribution of the enhanced field are used to compute the energy distributions of the detected electrons for 1-dimensional motion along the tip axis, $z$.

The incident THz field is given by [122],

$$
\begin{gathered}
A_{0}(0, t)=-\frac{F_{m} t_{\omega}}{C_{0}} \exp \left[-\left(1+\frac{1}{a} \tanh \frac{b t}{t_{\omega}}\right)\left(\frac{t}{t_{\omega}}\right)^{2}\right] \\
F_{0}(0, t)=-\frac{\mathrm{d} A_{0}(0, t)}{\mathrm{d} t}
\end{gathered}
$$

here $A_{0}(t)$ is the vector potential, $F_{m}$ and $t_{\omega}$ are the pulse maximum amplitude and the defined pulse duration, respectively $\left(\omega=\frac{\pi}{2 t_{\omega}}\right.$ is approximately the frequency of the field). $a=3, b=2, C_{0}=1.016$ and the field asymmetry is $2 / 3 . t_{\omega}=1 \mathrm{ps}$ is used 
in the simulation.

The temporally and spatially-varying $\mathrm{THz}$ electric field can be written as,

$$
F(z, t)=F_{0}(0, t)\left[(\gamma-1) \frac{R}{R+2 z(t)}+\exp \left(-\frac{(\ln 2)[z(t)]^{2}}{2 \omega_{h w h m}^{2}}\right)\right], \quad \gamma \simeq 0.06 \frac{\lambda}{R}
$$

here $\gamma$ is the enhancement factor, $R$ is the radius of the tip, $\omega_{h w h m} \simeq 1 \mathrm{~mm}$ is the $\mathrm{THz}$ beam focal size (intensity half-width-half-max), $\lambda \simeq 1.1 \mathrm{~mm}$ is the $\mathrm{THz}$ wavelength (calculated using the negative half cycle of the measured $\mathrm{THz}$ waveform $T \simeq 3.6 \mathrm{ps}$ ).

The tunneling emission current density is given by the standard Fowler-Nordheim equation [28],

$$
J(t)\left\{\begin{array}{l}
\propto\left|\gamma F_{0}(0, t)\right|^{2} \exp \left(-\frac{4 \sqrt{2 m_{e}} \Phi^{3 / 2}}{3 \hbar e\left|\gamma F_{0}(0, t)\right|}\right), \quad t>0 \\
=0, \quad t \leq 0
\end{array}\right.
$$

here $e, m_{e}, \hbar$ are the elementary charge, electron rest mass, and reduced Planck constant, respectively. $\Phi \simeq 4.5 \mathrm{eV}$ is the tungsten work function. The total electron yield during the $\mathrm{THz}$ pulse is therefore,

$$
Q_{t o t}=A \int_{0}^{\infty} J(t) \mathrm{d} t
$$

where $A$ is the area of the tip.

The energy of an electron emitted at time $t_{0}$ can be found by solving the 1D equation of motion,

$$
\begin{aligned}
& m_{e} \ddot{z}(t)=-e F(z, t) \\
& z\left(t_{0}\right)=0, \dot{z}\left(t_{0}\right)=0
\end{aligned}
$$

At the time it leaves the field, the electron has an energy $E_{t_{0}}\left(t_{\infty}\right)=\frac{m_{e}\left[\dot{z}\left(t_{\infty}\right)\right]^{2}}{2}$. Finally, the electron spectrum can be calculated,

$$
\left.\frac{\mathrm{d} P}{\mathrm{~d} E}\right|_{E=E_{0}}=\frac{Q\left(t\left(E=E_{0}\right)\right)}{Q_{t o t}}
$$



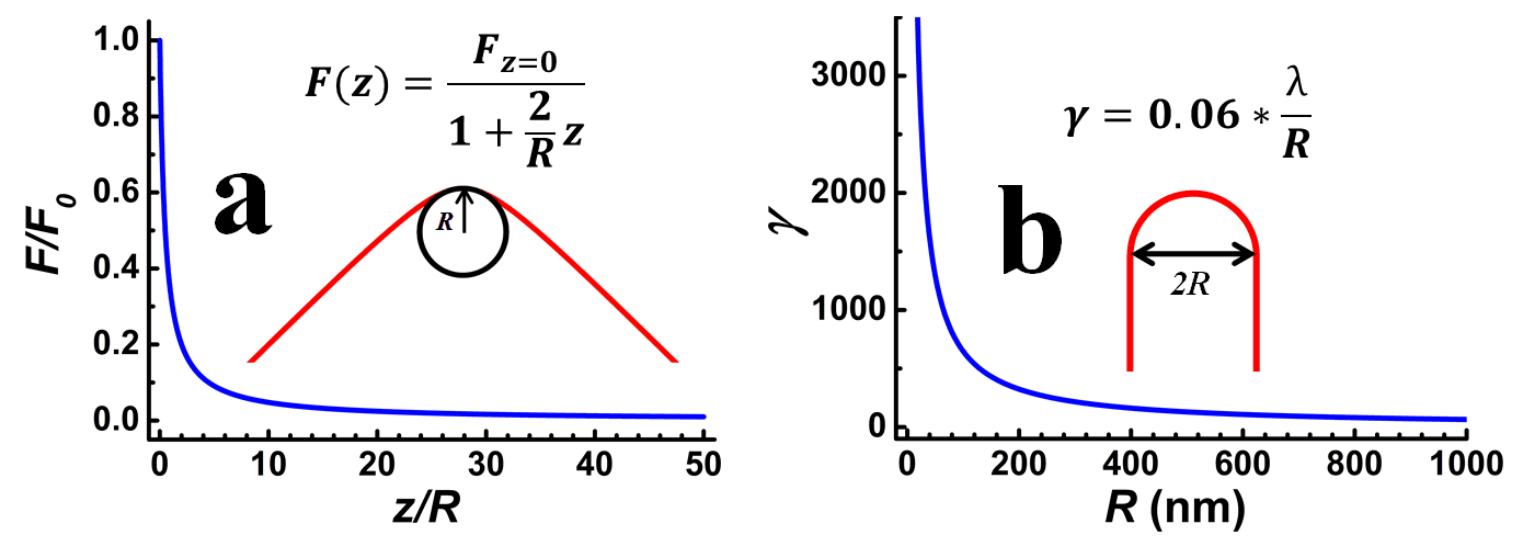

Figure J.1: a) Hyperboloidal model for field spatial decay. b) Hemisphere-capped cylinder model for field enhancement factor.
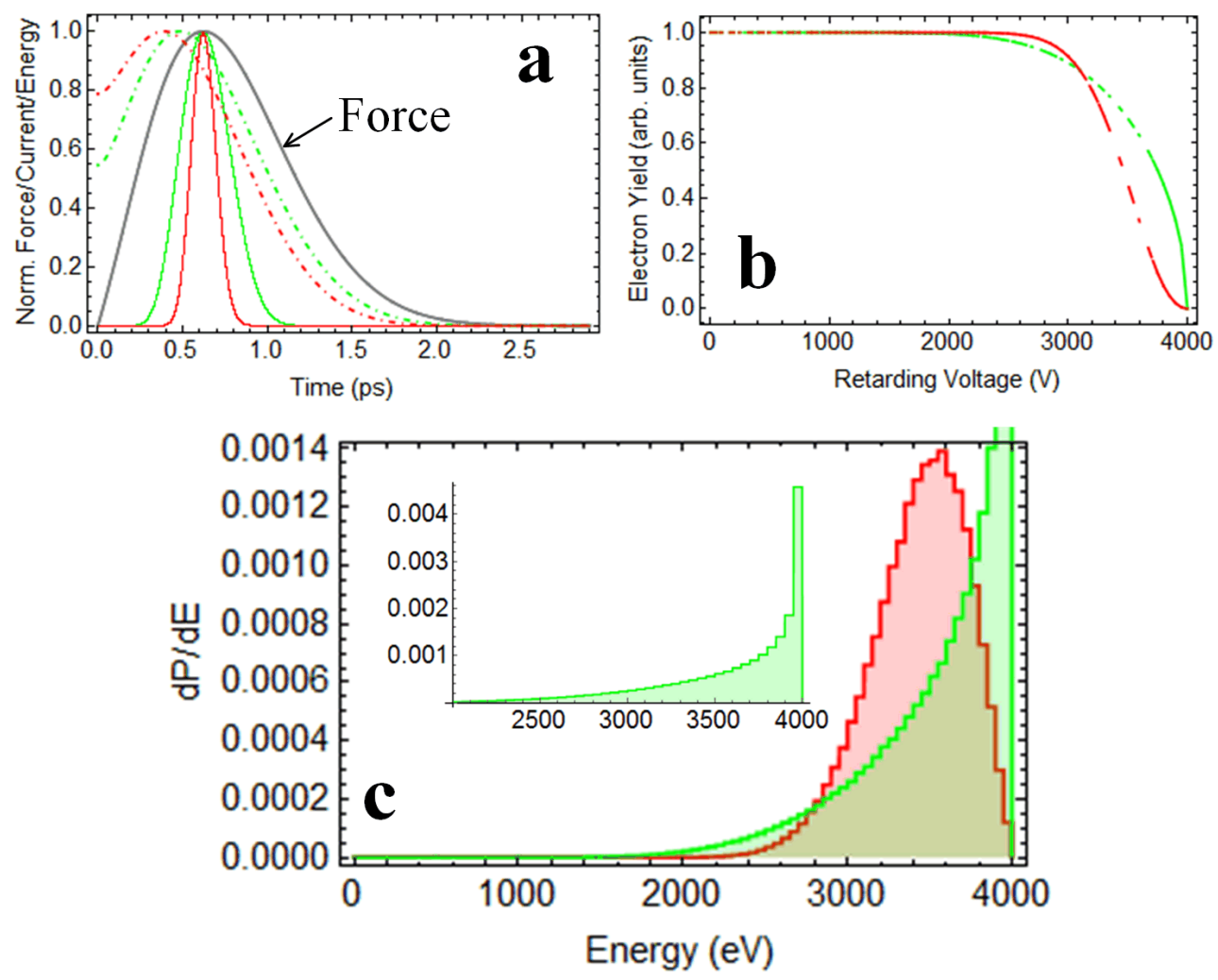

Figure J.2: Simulation results of field-emission electron energy spectra for two tips with radii $R=100 \mathrm{~nm}$ (green) and $R=800 \mathrm{~nm}$ (red) at fixed cutoff energy $E_{\text {cutoff }}=4000 \mathbf{e V}$. a) Normalized electron energy (dot-dashed line) and electron current density (solid line) as a function of emission time. b) Electron yield vs retarding voltage. c) Electron energy spectra. The off-scale part of the green curve is shown in the inset. The spectrum for the $R=100 \mathrm{~nm}$ tip exhibits a "spike" at the highest energy, however, this is not observed in the experiment. 